



\section{Living To The Fullest!}

Mindfulness-based interventions as public mental health interventions for depression 
Het onderzoek in dit proefschrift is mede mogelijk gemaakt door de Universiteit Twente en Dimence.

\section{UnIVERSITY OF TWENTE. Bimence}

Pots, W.T.M. (2016). Living To The Fullest! Mindfulness-based interventions as public mental health interventions for depression. Enschede, The Netherlands: University of Twente.

\section{(a) Wendy Pots}

Cover design and lay-out by Karina Dimitriu

Printed by Gildeprint Drukkerijen, The Netherlands.

Thesis, University of Twente, 2016

ISBN 978-90-365-4220-3

DOI $10.3990 / 1.9789036542203$ 


\title{
LIVING TO THE FULLEST!
}

MINDFULNESS-BASED INTERVENTIONS AS PUBLIC MENTAL HEALTH INTERVENTIONS FOR DEPRESSION

\author{
PROEFSCHRIFT \\ ter verkrijging van \\ de graad van doctor aan de Universiteit Twente, \\ op gezag van de rector magnificus, \\ Prof. dr. H. Brinksma, \\ volgens besluit van het College voor Promoties \\ in het openbaar te verdedigen \\ op vrijdag 18 november 2016 om 16.45 uur
}

DOOR

Wendy Theresia Maria Pots

geboren op 4 juni 1973

te Enschede 
Dit proefschrift is goedgekeurd door $1^{e}$ promotor Prof.dr. E.T. Bohlmeijer, $2^{e}$ promotor Prof.dr. K.M.G. Schreurs en co- promotor dr. P.A.M. Meulenbeek. 


\title{
Samenstelling promotiecommissie
}

\author{
Promotor: Prof.dr. E.T. Bohlmeijer \\ (Universiteit Twente) \\ Prof. dr. K.M.G. Schreurs \\ (Universiteit Twente; Roessingh Research \& Development) \\ Co-promotor: Dr.P.A.M. Meulenbeek \\ (Universiteit Twente; GGnet) \\ Leden: Dr. J.M. Bolier \\ (Trimbos-instituut) \\ Prof. dr. J.E.W.C. van Gemert - Pijnen \\ (Universiteit Twente) \\ Prof. dr. J.A.M. van der Palen \\ (Universiteit Twente; Medisch Spectrum Twente) \\ Prof. dr. J.J. Spijker \\ (Radboud Universiteit Nijmegen; Trimbos-instituut; ProPersona) \\ Prof. dr. Ph. Spinhoven \\ (Universiteit Leiden)
}



There's one thing that you are, that is you're the only one that is you ... Think about how much you weigh, and try to feel that weight in your seat right now, in your bottom right now. Parts in your feet and parts in your bum. Just try to feel your own weight, in your own seat, in your own feet. Okay? So if you can feel that weight in your body, if you can come back into the most personal identification, a very personal identification, which is: I am. This is me now. Here I am, right now. This is me now.

The only way we will ever know what it's like to be you is if you work your best at being you as often as you can, and keep reminding yourself that that's where home is.

Source: Bill Murray, March 25, 2015, answering the question "How does it feel to be you?"

Put on your lucky jersey, power pose and pump the jams.

Source: Found somewhere on the Internet 



\section{Table of Contents}

\section{CHAPTER 1}

General introduction

10

\section{CHAPTER 2}

The efficacy of mindfulness-based cognitive therapy as a public mental health intervention for adults with mild to moderate depressive symptomatology: a randomised controlled trial

\section{CHAPTER 3}

Effectiveness of online mindfulness-based interventions in improving mental health: a review and meta-analysis of randomised controlled trials

\section{CHAPTER 4}

Development of a web-based intervention for the indicated prevention of depression

\section{CHAPTER 5}

Acceptance and commitment therapy as a web-based intervention for depressive symptoms: randomised controlled trial

\section{CHAPTER 6}

How and for whom does web-based Acceptance and Commitment Therapy work? Mediation and moderation analyses of web-based ACT for depressive symptoms

\section{CHAPTER 7}

Web-based Acceptance and Commitment Therapy versus Expressive Writing and waitlist in depression: economic evaluation alongside a three-armed randomised trial

\section{CHAPTER 8}

General discussion

Summary in Dutch 


\section{CHAPTER 1}

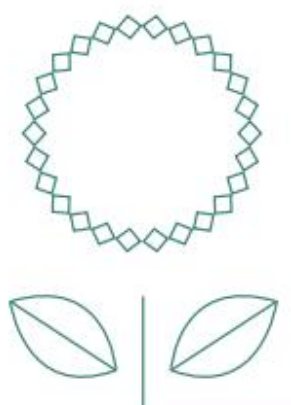

a.

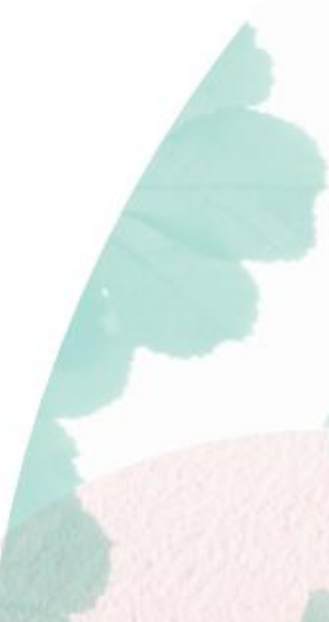

4he

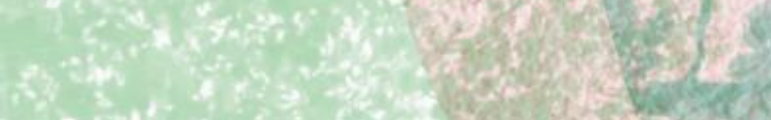

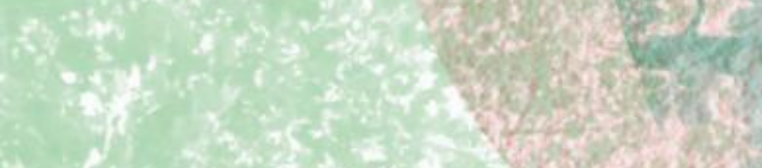

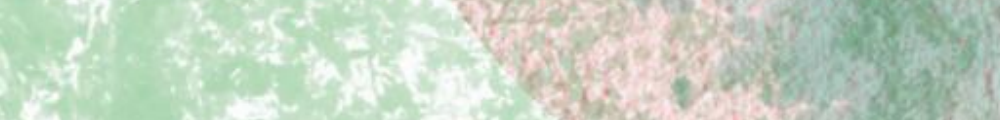
20

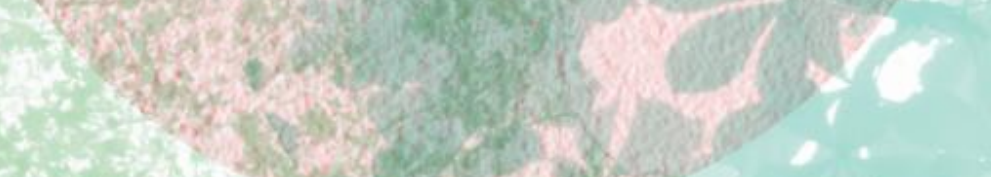




\section{General introduction}
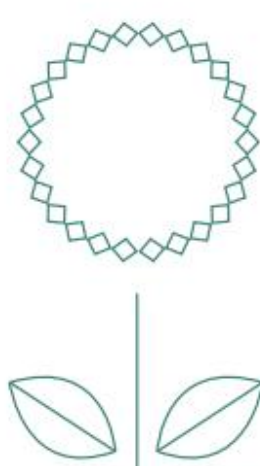


\section{What does it look like to really live your life to the fullest? \\ What does living to the fullest mean? \\ It means being in the present moment, living your life with all that it brings, the good and the bad, and doing the things that matter the most.}

In my work as a clinical psychologist I encounter many people with depression. They describe feeling sad, lonely and inactive, with all sorts of negative thoughts and feelings, and a life without energy and joy. They stop engaging in social activities and ruminate on their lives, which is expressed in negative feelings and thoughts that are constantly suppressing their inner strength. These thoughts encompass a range of feelings from worthlessness and existentialism to even suicide. The striking characteristic of most depressed people is that they see their lives as confined and empty and never think of their lives being full and meaningful.

The term depression appears in the Diagnostic and Statistical Manual of Mental Disorders as Major Depression Disorder (MDD; American Psychological Association, 2000). MDD is a disturbing disorder where people experience a depressed mood and/or a loss of interest or pleasure in activities, which, in turn, affects a person's self-esteem, work, relationships, sleep and eating habits and general health. In this thesis, the term MDD and depression are used interchangeably. The terms MDD and depression need to be distinguished from subclinical depression, which is having depressive symptoms without having MDD. According to the Global Burden of Disease Study, MDD is one of the leading causes of disease burden, causing disability and decreased quality of life (Ferrari et al., 2013; Murray et al., 2015; Whiteford et al., 2015). By the year 2030, depression is expected to rank first in disease burden in high-income countries (Mathers and Loncar, 2006). In The Netherlands, MDD is a prevalent disorder that has a large impact on quality of life, yielding high economic costs. Based on The Netherlands Mental Health Survey and Incidence Study-II (De Graaf et al., 2012a), the 12-month prevalence of MDD is estimated at 5.2\% of the population between 18 to 64 years, a total of around 550,300 people. The lifetime prevalence is $24.4 \%$ for women and $13.1 \%$ for men (De Graaf et al., 2012a). With each new episode of depression, the risk of another increases and can worsen the course of the disease (Kessing et al., 2004). In the first five years of recovery after a depressive episode, the risk of another episode is $70 \%$; for those with a history of three episodes, the risk rises to $90 \%$ (Judd, 1997; Mueller et al., 1999). When depressive symptoms persist more than two years, about $20 \%$ of the cases develop into chronic MDD (Keller and Boland, 1998). In The Netherlands, in 2011 approximately 280,000 new cases of depression were registered, which means that one out of two depressions every year is a new incidence (De Graaf et al., 2013; Nationaal Kompas 
Volksgezondheid, 2016). Also, people with depression have a 1.56 times higher risk of death and suicide, than people without depression (van den Akker et al., 2003). The economic costs (based on sick days and productivity) of depression is estimated to be $€ 2.6$ billion annually ( $D e$ Graaf et al., 2012b). From both a health and an economic perspective, the burden on society is significant.

A substantial proportion of adults experience mild to moderate depressive symptoms, without having MDD. Based on data from the Central Bureau of Statistics (Verwey and Houben-van Herten, 2013), 10.3\% of the adult population in The Netherlands experience mild to moderate depressive symptoms or a subclinical depression (reference year 2011). Having mild to moderate depressive symptoms is not only a risk factor for developing depression (Cuijpers and Smit, 2004; Muñoz et al., 2010), but also affects quality of life, resulting in increased service utilisation and high economic costs (Cuijpers et al., 2004; Cuijpers et al., 2007a; Smit et al., 2006). In The Netherlands, the costs of having mild to moderate depressive symptoms are considerable and approach those of depression; for example, in 2007, the costs were $\$ 160$ million per year per 1 million inhabitants, compared to \$192 million per year for depression (Cuijpers et al., 2007a).

Consequently, from a public health perspective, it is crucial to curtail the burden of disease by offering public mental health interventions that target people with mild to moderate depressive symptoms and potentially help prevent these people from slipping into MDD. Public mental health interventions are a complementary strategy to the in- and out-patient treatment of depression (Muñoz et al., 2010) as they focus on pro-actively offering preventive interventions for people with depressive symptoms who do not have MDD. Cuijpers et al. (2007b) conducted a meta-analysis of randomised controlled studies that examined the effects of psychological treatments for subclinical depression (that is, having depressive symptoms but not MDD). They concluded that the mean effect size at post-test was moderate $(p=0.42)$. The relative risk of developing MDD in subjects who received the intervention was reduced, compared with subjects in the control condition. In a follow-up meta-analysis and review, Cuijpers et al. (2008) found a mean incidence ratio of 0.78 , indicating a reduction of the incidence of depression by $22 \%$ in the experimental groups compared with the control group. In The Netherlands, a growing number of studies have examined the effects of public mental health interventions on the incidence of depression. To date, several meta-analyses and reviews have shown that most of the interventions are based on Cognitive Behavior Therapy (CBT), with effect sizes equivalent to pharmacotherapy and other effective treatments of depression (e.g. Andersson and Cuijpers, 2009; Barak et al., 2008; Cuijpers et al., 2008; Griffiths et al., 2010; Spek et al., 2007). Although research shows that public mental health interventions are effective and available, the impact on depression is low. Andrews et al. (2004) reported in their 
survey that about $66 \%$ of the burden of depression appears to not be averted or prevented. This indicates that although interventions are offered to treat or prevent depression, there remains room for improvement. Possible reasons of low impact are stigmatisation, high barriers to services, poor use of efficacious treatments (Cuijpers et al., 2010), and the financial coverage of more efficient treatments is not yet optimal (Andrews et al., 2004; Shafran et al., 2009). It is thus essential to develop public mental health interventions that are easily available, attractive and effective for people with depression.

The focus of this thesis is on the development and effectiveness of a specific subset of public mental health interventions that are easily acceptable and applicable, such as online or web-based interventions. Specifically, we focused on interventions that had a mental health promotion perspective that was appealing, namely interventions based on mindfulness and on Acceptance and Commitment Therapy (ACT). This introductory chapter starts with a short overview of a new era of public mental health interventions that encompass these mindfulness-based interventions (MBIs). Thereafter, we discuss the effectiveness of MBIs in general and Mindfulness-Based Cognitive Therapy (MBCT) and ACT specifically, followed by a discussion of available online MBIs. Additionally, an extensive description of the web-based ACT intervention "Living to the Full" is provided. This chapter concludes with an outline of the studies that were conducted within the context of this thesis.

\section{A new era of public mental health interventions}

As we know that mild to moderate depressive symptoms are a risk factor for the development of full-blown depression (Cuijpers and Smit, 2004; Muñoz et al., 2010), it is vital to offer effective and acceptable treatments by means of prevention or early intervention that not only focus on the reduction of psychological distress, but that also promote positive mental health. In 2005, the World Health Organization defined mental health as a state of well-being in which the individual realises his or her own abilities, can cope with the normal stresses of life, can work productively, and is able to make a contribution to his or her community (World Health Organization, 2005, p. 2). Studies have shown that well-being reduces the risk of developing psychopathology later on (Keyes et al., 2010; Lamers et al., 2015; Wood and Joseph, 2010). Traditionally, in clinical psychology the main focus is on mental illness, where positive mental health is seen as the counterpart of psychopathology. The two-continua model (Keyes, 2005) provides an alternative perspective to this traditional view, with one continuum reflecting the presence or absence of psychopathology, and the other the presence or absence of positive mental health. In this two-con- 
tinua model, mental illness and well-being are distinct but related continua (Keyes et al., 2008; Lamers et al., 2011). This model embraces that while an individual with few symptoms of psychopathology may experience high positive mental health, he or she may also experience low or no positive mental health. Growing evidence now shows that decreases in positive mental health (as well as poor positive mental health) can predict future mental disorders (Keyes et al., 2010; Wood and Joseph, 2010). This perspective gives rise to a new era of public mental health interventions based on mental health promotion (Bolier et al., 2013; Jané-Llopis et al., 2005; Vella-Brodrick, 2013).

Central in these new public mental health interventions are the processes of mindfulness, psychological flexibility, and emotion regulation (Hayes, 2004; Hayes et al., 2004; Öst, 2008). These processes (or lack thereof), are linked to psychopathology in general, and specifically to depression (Aldao and Nolen-Hoeksema, 2010; Hayes et al., 2006). Mindfulness refers to the ability to observe thoughts, bodily sensations, or feelings in the present moment with an open and accepting orientation toward one's experiences (Bishop et al., 2004; Kabat-Zinn, 1990).

Psychological flexibility is defined as the willingness to come into contact with one's own private experiences (thoughts, feelings and bodily sensations), recognise one's values, and commit to behaviours that are consistent with those values (Hayes et al., 2012). One of the core processes that contribute to psychological inflexibility is experiential avoidance, i.e. the persistent and generally fruitless attempts to avoid unwanted private experiences such as thoughts, feelings and bodily sensations. Experiential avoidance causes behavioural constriction and is associated with psychopathology (for a review see e.g. Chawla and Ostafin, 2007). This psychological and behavioural inflexibility prevents people from engaging in valued life activities, and thereby inhibits them from leading a valued life (Hayes et al., 2006). Emotion regulation refers to the ability to experience and differentiate emotions, both positive and negative, as well as the ability to regulate (strong) emotions (Gratz and Roemer, 2004). It may prevent people from engaging in maladaptive behaviours that set the stage for negative emotional experiences, which can subsequently persist and develop into depression (e.g. Chiesa et al., 2013; Farb et al., 2012; Hölzel et al., 2011). Emotion regulation can be seen to have a broader context to it, comprising of both psychological flexibility and mindfulness. Emotion regulation has also been identified as a transdiagnostic risk factor for depression, specifically, and emotion disorders broadly (Aldao and Nolen-Hoeksema, 2010; Hofmann et al., 2012; Norton and Paulus, 2015). 


\section{Mindfulness-based interventions}

Interventions based on training in mindfulness skills are becoming increasingly popular. Since the 1970 s a number of therapeutic applications have integrated or used the practice of mindfulness to help people who are experiencing a variety of psychological conditions such as stress, worry, anxiety and depression. Through facilitating awareness and non-judgmental acceptance of moment-to-moment experiences, these mindfulness-based interventions (MBIs) are assumed to alleviate intense emotional states. Examples of interventions incorporating mindfulness exercises are Mindfulness-Based Stress Reduction (MBSR; Kabat-Zinn, 1982; Kabat-Zinn, 1990), Mindfulness-Based Cognitive Therapy (MBCT; Segal et al., 2002), Dialectical Behaviour Therapy (DBT; Linehan, 1993), and Acceptance and Commitment Therapy (ACT; Hayes et al., 1999). In the past two decades, an increasing number of studies have addressed the non-clinical and clinical utility of these interventions (e.g. Baer, 2003; Cavanagh et al., 2014; Chiesa and Serretti, 2011; Keng et al., 2011). As evidenced by a substantial number of meta-analyses, MBIs have proven effective in reducing psychological distress, most notably anxiety and depression, and improving well-being and quality of life within a broad range of populations, including healthy populations (Chiesa and Serretti, 2009; Khoury et al., 2015), individuals with mental disorders (Chiesa and Serretti, 2011; Klainin-Yobas et al., 2012; Kuyken et al., 2016; McCarney et al., 2012; Piet and Hougaard, 2011; Strauss et al., 2014; Vøllestad et al., 2012), and individuals suffering from chronic somatic illnesses (Abbott et al., 2014; Bohlmeijer et al., 2010; Cramer et al., 2012; Lauche et al., 2013; Ledesma and Kumano, 2009; Veehof et al., 2011; Zainal et al., 2013). The effect-size estimates vary between moderate to large in comparison with waitlist controls and small to moderate in comparison with other psychological treatments. Also, a meta-analysis by Khoury et al. (2013), based on 209 studies enrolling 12,145 participants with a variety of disorders, suggests that the effect size of MBIs do not differ from traditional CBT, behavioural therapies, or pharmacological treatments.

Currently, mindfulness practices have been incorporated into various therapies in the field of mental health care, including MBCT (Segal et al., 2002) and ACT (Hayes et al., 1999), which are the focus of this thesis. Both MBCT and ACT target processes such as mindfulness, psychological flexibility, and emotion regulation.

\section{MBCT}

$\mathrm{MBCT}$ is a group-based training that combines CBT for depression with training in mindfulness meditation to prevent relapse for people with major depressive disorder in remission (Segal et al., 2002). MBCT was developed in 1970 and based upon Mindfulness-Based Stress Reduction (MBSR), developed by John Kabat-Zinn (1990). 
Kabat-Zinn developed MBSR as an intervention to alleviate stress by providing a training in mindfulness meditation and yoga. While intrigued by this training program, William Teasdale and his colleagues found it unsuitable for the depressed patients with whom they were working. As a result they incorporated components of MBSR with techniques from CBT (Beck et al., 1979). Unlike CBT, the emphasis of $\mathrm{MBCT}$ is not on changing the content of thoughts, but rather on changing the awareness of and relationship to thoughts, feelings and bodily sensations. Examples of statements that facilitate this decentering are: "Thoughts are not facts" and "I am not my thoughts". The core of MBCT is learning to become aware of and disengage from habitual dysfunctional (cognitive) routines, to stop reacting automatically to internal experiences, and to act more 'mindfully'. Through the practice of mindfulness exercises, such as the body-scan, breathing meditation, and yoga exercises, people are taught to become more aware of their thoughts, feelings and bodily sensations, and to relate differently to them. MBCT is regularly delivered as an 8-week program, featuring 2-hour sessions with up to 12 people who are recovered from an episode of recurrent depression. The program consists of psycho-education and several informal and formal meditation exercises, complemented by 45 minutes of daily meditation as a homework exercise.

The model underlying MBCT (Segal et al., 2002) describes that people who have been previously depressed are characterized by a greater vulnerability to states of low mood, which can lead to the reactivation of patterns of negative thinking similar to those of previous episodes (see also Nolen-Hoeksema, 2000; Olatunji et al., 2013). Laboratory studies support this theoretical model by showing that after inducing a sad mood, depressed patients revert to a depressive information processing style (Lau et al., 2004). Increased mindfulness is thought to enable early detection of relapse-related patterns of negative thinking, feelings and bodily sensations, and, consequently, to the disengagement of relatively automatic ruminative thoughts patterns that otherwise might fuel the relapse process. As MBCT targets cognitive reactivation, mindfulness and compassion are proposed to be important mediators of change (Kuyken et al., 2010). Developing mindfulness skills, as a way to cultivate meta-awareness (or decentering), acceptance, and self-compassion, can help disintegrate associative networks that trigger the risk for relapse. In addition, a number of theoretical models have suggested transdiagnostic mechanisms, such as alterations in attention, memory specificity, emotion regulation, and momentary positive and negative affect could play a role in MBCT (e.g. Batink et al., 2013; Chambers et al., 2008; Chiesa et al., 2013; De Raedt et al., 2012; Geschwind et al., 2012; Hargus et al., 2010; Hölzel et al., 2011; Williams, 2010). 


\section{ACT}

Acceptance and Commitment Therapy (ACT) is an empirically-based behavioural cognitive therapy that uses acceptance and mindfulness strategies together with commitment and behaviour change strategies (Hayes et al., 2012). The model underlying ACT is Relational Frame Theory (RFT), which has its roots in functional contextualism (Biglan and Hayes, 1996, Hayes et al., 2001). Experiments based on RFT show that people are prone to react to verbal and symbolic representations of stimuli in the same way as the react to real life stimuli, because of their innate verbal capacity. Classical conditioning creates learned behaviour, causing relational frames to unconsciously arise. These frames consist of stimuli, concepts and symbols that refer to stimuli, functional classes of responses and the consequences of such responses. For example, a depressed person can, for instance, have negative thoughts when they attend a party (i.e. "I'm not worthy enough to contact another person"), and act upon these thoughts as if they are facts by isolating themselves. In this way, the person can attach psychological functions (e.g., worthlessness, loneliness, isolation) to previously neutral events (e.g., parties). RFT has important implications for clinical practice in that relational frames create stimulus function shifts that cause us to react to our thoughts as facts. In addition, negative thoughts and feelings cannot be extinguished; they are part of the relational frame and are irrevocably activated when the frame is activated. For instance, when a depressed person thinks "I'm doing OK", this thought refers to the frame of depressed thoughts and feelings, which subsequently activates the frame. In this way, the more a person tries to avoid having depressed thoughts or feelings, the more these feelings and thoughts are activated. The central aim of ACT is to create psychological flexibility by teaching skills that increase an individual's willingness to come into fuller contact with their experiences, recognise a person's values in life, and commit to behaviours that are consistent with those values. Psychological inflexibility is associated with greater depression among adults (Bond et al., 2011) and has been identified as a process underlying the development of depression (Hayes et al., 2006; Kashdan and Rottenberg, 2010). ACT encourages people to think and feel, without attempting to change or suppress their thoughts and feelings, and helps people move in a valued direction, with all of their experiences and learned behaviour patterns. Suppression has been subsumed under the more general term of experiential avoidance, which is defined as the unwillingness to experience negatively evaluated feelings, physical sensations, and thoughts (Aldao and Nolen-Hoeksema, 2010; Hayes et al., 2006; Kashdan and Rottenberg, 2010). Thus, the goal of ACT is to increase the ability of the individual to act in accordance with personal values in the presence of life adversities. It relies heavily on metaphors, stories, experiential exercises, behavioural tasks, and other forms of non-literal forms of language. 
The six core processes that underlie psychological flexibility are represented in the hexaflex model (see Figure 1). These six processes form part of the two major processes within the ACT perspective. The four processes on the left of Figure 1-contact with the present moment, acceptance, defusion, and self-as-context - together teach acceptance and mindfulness skills. The four on the right of Figure 1 - contact with the present moment, self-as-context, values, and committed action - together teach commitment and behaviour skills.

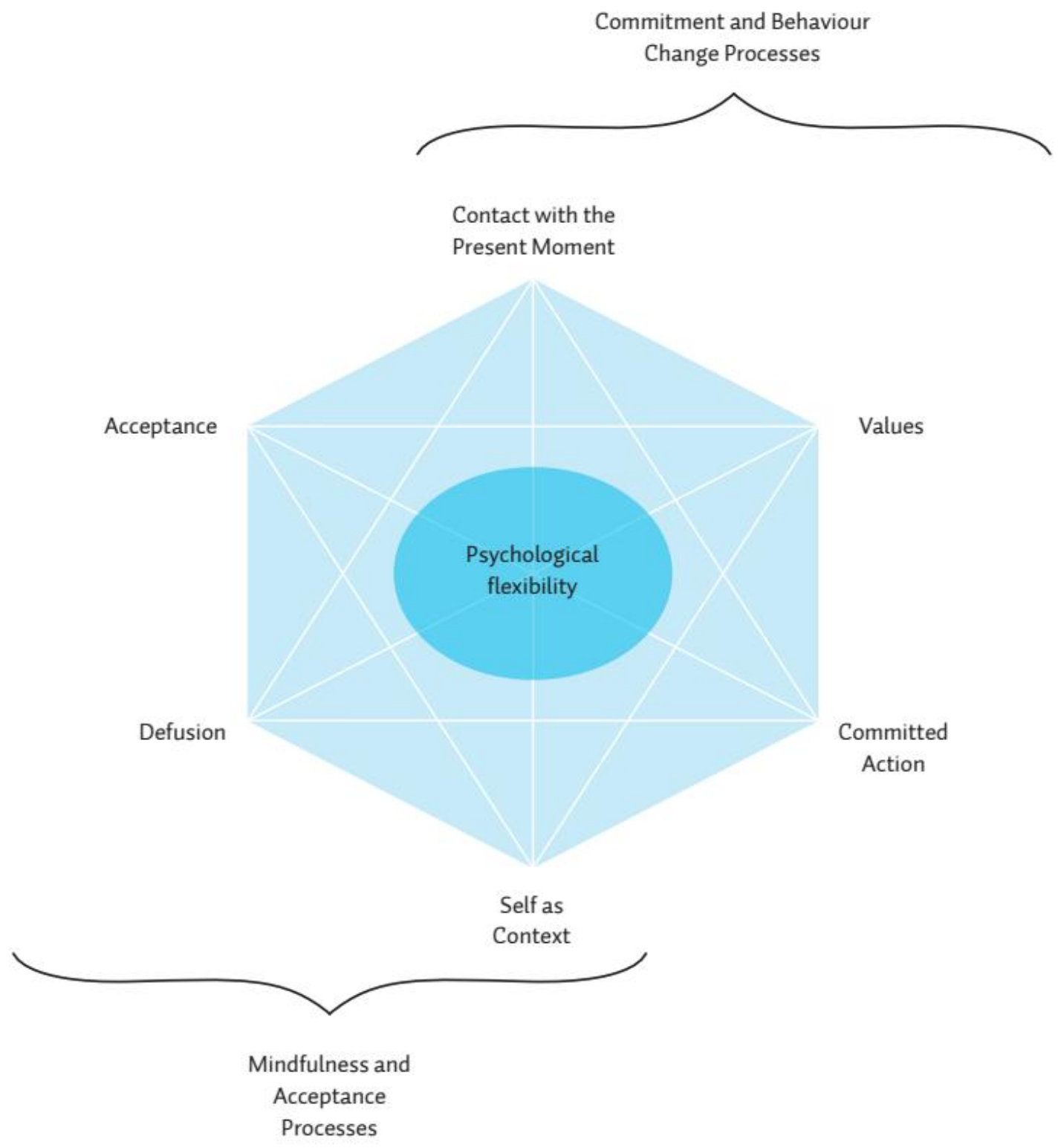

Figure 1 - The core processes of psychological flexibility in the ACT hexaflex (Hayes et al., 2012, p. 63). 
Each of the six processes is explained below:

1) Contact with the present moment, or in other words 'being present', is the process that is most similar to mindfulness. It refers to a stance of non-judgmental presence in the here-and-now, without being consumed by the past or future. The goal is to experience the world as it is, so that one's behaviour is more flexible and thus actions are more consistent with the values that they hold.

2) Acceptance is presented as an alternative to experiential avoidance. It encompasses a non-judgmental willingness to experience all the feelings, thoughts and bodily sensations that accompany depression without attempting to change their frequency or form. However, acceptance is not an end in itself. Rather, acceptance is encouraged as a way to stop the unsuccessful struggle of controlling the depressive symptoms such as negative thoughts or a low self-esteem, and redirect one's attention towards values-based action.

3) Cognitive defusion techniques focus on altering the undesirable functions of negative thoughts, feelings and other private events, rather than trying to change and act upon the content. In other words, people are encouraged to reduce the literal quality of the thought, by creating 'distance' and weakening the tendency to treat the thought as what it refers to ("I am no good") rather than what it is directly experienced to be (e.g., the thought "I am no good"). The goal of defusion is to decrease the believability of, or attachment to, negative thoughts or private events.

4) Self as context is a process that promotes being aware of the difference between 'self-as-content' - a sense of self composed of all the roles, ideas, thoughts, and labels we have about our self, and 'self-as-context' - a sense of self that is aware and observes physical and psychological events that take place in our lives. From this observing perspective, it becomes possible to defuse from over-identification with unhelpful ideas, thoughts or labels about oneself.

5) Values are intrinsically motivated qualities of a meaningful life, which are personally valuable and of essential importance. They are freely chosen life directions in various domains (e.g., family, career, social activities, spirituality). They undermine verbal processes that might lead to choices based on avoidance, social compliance, or fusion (e.g., "I should value X" or "A good person would value $Y$ " or "My mother wants me to value Z').

6) Committed action refers to a values-based action designed to create a pattern of action that is itself values-based. A person is engaging in a committed action when he of she sees a divergence and chooses to redirect his or her behaviour so that is it values-consistent. ACT uses homework assignments and behavioural exercises to build these patterns of flexible and effective values-based behaviour. 


\section{Effectiveness of MBIs}

Traditionally, MBCT was developed and used for the treatment of people with MDD in remission. Two large randomised controlled trials found that for patients with three or more previous episodes of depression, MBCT significantly reduced the risk of relapse compared to the treatment as usual (Ma and Teasdale, 2004; Teasdale et al., 2000). This effect has been confirmed in two extensive meta-analyses (Kuyken et al., 2016; Piet and Hougaard, 2011). Moreover, in a recently published randomised controlled dismantling trial, Williams et al. (2014) confirmed these studies, adding that the number of episodes is a marker for those with greater vulnerability due to a history of childhood trauma and adversity. Of particular interest however, is that $M B C T$ has recently been adapted to different clinical settings and different disorders and conditions, with increasing empirical data on the effectiveness of these adapted MBCT on symptoms of depression (e.g. Hofmann et al., 2010; Strauss et al., 2014).

ACT has been shown to be effective for a range of disorders and other problems, such as depression and anxiety, stress, and chronic pain (most recently, A-Tjak et al., 2015; Öst, 2014; Ruiz, 2010). Specifically, for depression moderate effect sizes post-treatment and follow-up have been shown. In addition, Hacker et al. (2016) performed a sequential meta-analysis techniques to critically appraise the sufficiency of the existing evidence base, and found that there is now sufficient evidence of at least a moderate effect of ACT for depression.

\section{Web-based MBIs}

Given the rapid development of information technologies in more recent years it is not surprising that MBIs - like other psychotherapeutic interventions - are increasingly delivered through the Internet. According to Barak (2009), a web-based intervention is:

\footnotetext{
.. a primarily self-guided intervention program that is executed by means of a prescriptive online program operated through a website and used by consumers seeking health- and mental-health related assistance. The intervention itself attempts to create positive change and or improve/enhance knowledge, awareness, and understanding via the provision of sound health-related material and use of interactive web-based components (p. 5).
}

A typical web-based intervention is meant to be used once a week, is set-up as modular, has updates once a week, lasts for 10 weeks, and has some interaction with the program itself and/or a counsellor (Kelders et al., 2012). Web-based interventions 
can have a number of advantages over face-to-face interventions: 1) web-based interventions are easily accessible, without long waiting lists; 2) people can use the intervention $24 / 7$ in their own environment, thereby saving traveling time and enabling people to work at their own pace; 3 ) users can remain anonymous and do not have to adopt a patient role; 4) it does not necessarily require involvement of a therapist educated in mindfulness; and 5 ) it is less costly (Andersson and Titov, 2014; Cuijpers et al., 2010). Moreover, a cross-sectional survey among 500 adults showed that a high percentage of people prefer individual and web-based formats for mindfulness meditation interventions above group formats (Wahbeh et al., 2014). They found that the Internet was the first choice format for $43 \%$ of the participants, suggesting that online MBIs are an acceptable alternative to face-to-face formats. Hence, web-based interventions provide an opportunity to overcome the reasons for the low impact of public mental health interventions for depression (Andersson and Titov, 2014; Andrews et al., 2010; Cuijpers et al., 2010; Marks and Cavanagh, 2009; Marks et al., 2007; Shafran et al., 2009).

To date, some randomised controlled trials have provided empirical evidence for the effectiveness of web-based MBIs in the context of mental health and well-being. The research is still, however, in its infancy, both in the context of MBCT (Boettcher et al., 2014; Dowd et al., 2015; Ly et al., 2014b) and ACT (Bricker et al., 2013; Bricker et al., 2014; Buhrman et al., 2013; Hesser et al., 2014; Lappalainen et al., 2014; Lappalainen et al., 2015; Levin et al., 2014; Ly et al., 2014a; Pots et al., 2015; Trompetter et al., 2014).

\section{Living to the Full}

"Living to the Full" is an intervention based on an ACT protocol with an explicit focus on mindfulness (Bohlmeijer and Hulsbergen, 2013). As both a group course and as a bibliotherapy intervention with e-mail support, this self-help intervention has proven to be effective in reducing depressive and anxiety symptoms, and improving positive mental health, psychological flexibility and mindfulness (Bohlmeijer et al., 2011; Fledderus et al., 2012; Fledderus et al., 2010). Based on these studies, we developed a web-based version of the intervention to enhance participation by creating a userfriendly application, that best fit the values of the stakeholders (Kelders et al., 2013).

The web-based ACT intervention is comprised of nine online modules, which can be completed in nine to twelve weeks. The modules of the web-based intervention are based on the six core processes of ACT that together promote psychological flexibility (see Figure 1), with additional mindfulness exercises. The nine modules of the web-based intervention are divided into three major parts, which reflect the six core processes of $A C T: 1$ ) an introduction to $A C T, 2$ ) ACT resources, and 3) ACT 
in daily life. In the first part of the web-based intervention, participants reflect on their avoidance and control strategies, and ascertain whether these are effective in the long run. In the second part, participants practice cognitive defusion and self as context as they learn how to stay in contact with their present experiences without trying to avoid or control them. In the third part, the focus is on creating awareness of one's own personal values and taking action based on these values. An additional focus of the intervention is relapse prevention, which includes self-management and action plans. Each module uses experiential exercises and metaphors to illustrate the ACT processes.

Mindfulness is taught throughout the web-based intervention, during which a variety of exercises are used to enhance awareness of an observing self and to teach people to take a stance from their thoughts and feelings without acting on them. The mindfulness exercises are a combination of mindfulness meditation exercises, based on MBSR (Kabat-Zinn, 1990), as well as exercises specifically focused on the processes of ACT. Participants are encouraged to practice daily mindfulness exercises, which last, on average, 10-15 minutes, and are provided as audio files (downloadable within the web-based intervention). In addition, for our study, weekly e-mail counselling by a trained graduate clinical psychology student was offered to the participants, providing them with personal feedback, encouragement and support.

The web-based intervention "Living to the Full” was designed using the Center for eHealth Research and Disease Management (CeHRes) Roadmap to achieve a userfriendly application that would fit the values of the stakeholders and could evaluate the process of development (Kelders et al., 2013). Furthermore, an effort was made to include persuasive technology in the design of the web-based intervention by using technology to motivate people to adhere to the intervention. Persuasive design has been shown to be positively related to adherence (Kelders et al., 2012). Examples from the web-based ACT intervention include the implementation of text messages tailored to the content of the module being worked on, tailored stories of former participants sharing their stories and experiences related to a module, multi-media messages, interactive exercises, and an option to personalise the homepage.

\section{An overview of this thesis}

The current challenge is to curtail the burden of depression by pro-actively offering interventions for people who do not yet have MDD. The opportunity to use a positive framework, promote positive mental health, and reduce psychological distress can potentially improve the impact of public mental health interventions. Although public mental health interventions based on mindfulness have such a potential, 
effectiveness studies remain lacking. Therefore, to broaden the impact of public mental health interventions on depression, it is worthwhile to study if MBIs can be delivered online in a (cost-)effective way.

The overall aim and central question of this thesis was whether public mental health interventions based on mindfulness and acceptance can be effective as mental health promotion for adults with mild to moderate depressive symptoms. In this thesis, three main goals were formulated, leading to six research questions and, consequently, six studies to answer these questions.

The first goal was to study whether MBCT could also be effective for people with depressive symptomatology, beyond MDD in remission. The research question was whether a community-based MBCT intervention could be effective as a public mental health intervention for people with mild to moderate depressive symptomatology. This question is answered in Chapter 2, where we describe a large, pragmatic, multi-site randomised controlled trial that evaluated a MBCT that was offered as a community-based group course with brief mindfulness exercises, compared to a waiting list control group. Additional analyses were performed to study the mediating effects of mindfulness and psychological flexibility on depressive symptoms.

The second goal was to study whether web-based or online public mental health interventions based on mindfulness were effective. The research question was what is the overall effect of all available online mindfulness-based interventions on mental health outcomes. In Chapter 3 , we describe our meta-analysis and systematic review on the impact of web-based or online MBIs. The overall effect size of online MBIs was estimated for depressive symptoms, anxiety symptoms, stress, and well-being, and additional analyses were performed to explore possible moderators on outcome.

Our third goal was to broaden the availability of online MBIs in a (cost-)effective way, by developing "Living to the Full" into a web-based intervention based on ACT and mindfulness, for adults with mild to moderate depressive symptoms. In Chapter 4, we discuss our findings regarding our third research question, namely whether we could develop a web-based intervention that was acceptable for intended users. We developed the web-based ACT intervention following the CeHRes Roadmap, using a human-centered approach and implementing persuasive technologies into the design. The fourth research question was whether the web-based ACT intervention could be an effective and applicable public mental health intervention for adults with depressive symptoms. Chapter 5 focuses on the effectiveness of this web-based ACT intervention in a large randomised controlled trial. In the randomised controlled trial, the web-based ACT intervention was not only compared to a waiting list control group but also to an active control group. The fifth research question aimed to evaluate how and for whom the web-based ACT intervention was effective. In Chapter 6, we describe the mediational analyses that we performed in order to study whether the 
presumed active ingredients were indeed mediating the effects of the intervention on depressive symptoms. Also, to address the question for whom a web-based ACT intervention might be most appropriate, we looked at possible moderators and predictors. Finally, our sixth research question was whether the web-based ACT intervention was cost-effective, compared to both control groups. In Chapter 7 , our cost-effectiveness and cost-utility analyses is described in which we studied whether the web-based ACT intervention might lead to better health outcomes against lower costs.

In Chapter 8, the major findings of this thesis are summarized and discussed, limitations and suggestions for future research are provided, and we expound on the implications of this research for clinical practice. 


\section{References}

A-Tjak, J. G. L., Davis, M. L., Morina, N., Powers, M. B., Smits, J. A. J. \& Emmelkamp, P. M. G. (2015). A meta-analysis of the efficacy of acceptance and commitment therapy for clinically relevant mental and physical health problems. Psychotherapy and Psychosomatics 84, 30-36.

Abbott, R. A., Whear, R., Rodgers, L. R., Bethel, A., Thompson Coon, J., Kuyken, W., .. . Dickens, C. (2014). Effectiveness of mindfulness-based stress reduction and mindfulness based cognitive therapy in vascular disease: A systematic review and meta-analysis of randomised controlled trials. Journal of Psychosomatic Research 76, 341-351.

Aldao, A. \& Nolen-Hoeksema, S. (2010). Specificity of cognitive emotion regulation strategies: A transdiagnostic examination. Behaviour Research and Therapy 48, 974-983.

American Psychological Association (2000). Diagnostic and Statistical Manual of Mental Disorders, Fourth Edition-Text Revised. American Psychological Association: Washington, DC.

Andersson, G. \& Cuijpers, P. (2009). Internet-based and other computerized psychological treatments for adult depression: A meta-analysis. Cognitive Behaviour Therapy 38, 196-205.

Andersson, G. \& Titov, N. (2014). Advantages and limitations of Internet-based interventions for common mental disorders. World Psychiatry 13, 4-11.

Andrews, G., Cuijpers, P., Craske, M. G., McEvoy, P. \& Titov, N. (2010). Computer therapy for the anxiety and depressive disorders is effective, acceptable and practical health care: A meta-analysis. PLOS ONE 5, el3196.

Andrews, G., Issakidis, C., Sanderson, K., Corry, J. \& Lapsley, H. (2004). Utilising survey data to inform public policy: Comparison of the cost-effectiveness of treatment of ten mental disorders. British Journal of Psychiatry 184, 526-533.

Baer, R. A. (2003). Mindfulness training as a clinical intervention: A conceptual and empirical review. Clinical Psychology: Science and Practice 10, 125-143.

Barak, A., Hen, L., Boniel-Nissim, M. \& Shapira, N. (2008). A comprehensive review and a meta-analysis of the effectiveness of internet-based psychotherapeutic interventions. Journal of Technology in Human Services 26, 109-160.

Barak, A., Klein, B. \& Proudfoot, J.G. (2009). Defining internet-supported therapeutic interventions. Annuals of Behavioral Medicine 38, 4-17.

Batink, T., Peeters, F., Geschwind, N., van Os, J. \& Wichers, M. (2013). How Does MBCT for Depression Work? Studying Cognitive and Affective Mediation Pathways. PLoS ONE 8.

Beck, A. T., Rush, A. J., Shaw, B. F. \& Emery, G. (1979). Cognitive therapy for depression. Guilford Press: New York.

Biglan, A. \& Hayes, S. C. (1996). Should the behavioral sciences become more pragmatic? the case for functional contextualism in research on human behavior. Applied and Preventive Psychology 5, 47-57.

Bishop, S. R., Lau, M., Shapiro, S., Carlson, L., Anderson, N. D., Carmody, J., ... Devins, G. (2004). Mindfulness: A proposed operational definition. Clinical Psychology: Science and Practice n, 230-241.

Boettcher, J., Åström, V., Påhlsson, D., Schenström, O., Andersson, G. \& Carlbring, P. (2014). InternetBased Mindfulness Treatment for Anxiety Disorders: A Randomized Controlled Trial. Behavior Therapy 45, 241-253.

Bohlmeijer, E. \& Hulsbergen, M. (2013). A beginner's guide to mindfulness: live in the moment. Open University Press: Oxford.

Bohlmeijer, E., Prenger, R., Taal, E. \& Cuijpers, P. (2010). The effects of mindfulness-based stress reduction therapy on mental health of adults with a chronic medical disease: A meta-analysis. Journal of Psychosomatic Research 68, 539-544.

Bohlmeijer, E. T., Fledderus, M., Rokx, T. A. J. J. \& Pieterse, M. E. (2011). Efficacy of an early intervention based on acceptance and commitment therapy for adults with depressive symptomatology: Evaluation in a randomized controlled trial. Behaviour Research and Therapy 49, 62-67. 
Bolier, L., Haverman, M., Westerhof, G. J., Riper, H., Smit, F. \& Bohlmeijer, E. (2013). Positive psychology interventions: A meta-analysis of randomized controlled studies. BMC Public Health 13.

Bond, F. W., Hayes, S. C., Baer, R. A., Carpenter, K. M., Guenole, N., Orcutt, H. K., ... Zettle, R. D. (2011). Preliminary Psychometric Properties of the Acceptance and Action Questionnaire-II: A Revised Measure of Psychological Inflexibility and Experiential Avoidance. Behavior Therapy 42, 676-688.

Bricker, J., Wyszynski, C., Comstock, B. \& Heffner, J. L. (2013). Pilot randomized controlled trial of web-based acceptance and commitment therapy for smoking cessation. Nicotine and Tobacco Research 15, 1756-1764.

Bricker, J. B., Mull, K. E., Kientz, J. A., Vilardaga, R., Mercer, L. D., Akioka, K. J. \& Heffner, J. L. (2014). Randomized, controlled pilot trial of a smartphone app for smoking cessation using acceptance and commitment therapy. Drug and Alcohol Dependence 143, 87-94.

Buhrman, M., Skoglund, A., Husell, J., Bergström, K., Gordh, T., Hursti, T., ... Andersson, G. (2013). Guided internet-delivered acceptance and commitment therapy for chronic pain patients: A randomized controlled trial. Behaviour Research and Therapy 51, 307-315.

Cavanagh, K., Strauss, C., Forder, L. \& Jones, F. (2014). Can mindfulness and acceptance be learnt by self-help?: A systematic review and meta-analysis of mindfulness and acceptance-based self-help interventions. Clinical Psychology Review 34, 118-129.

Chambers, R., Lo, B. C. Y. \& Allen, N. B. (2008). The impact of intensive mindfulness training on attentional control, cognitive style, and affect. Cognitive Therapy and Research 32, 303-322.

Chawla, N. \& Ostafin, B. (2007). Experiential avoidance as a functional dimensional approach to psychopathology: An empirical review. Journal of Clinical Psychology 63, 871-89o.

Chiesa, A. \& Serretti, A. (2009). Mindfulness-based stress reduction for stress management in healthy people: A review and meta-analysis. Journal of Alternative and Complementary Medicine 15, 593-600.

Chiesa, A. \& Serretti, A. (2011). Mindfulness based cognitive therapy for psychiatric disorders: A systematic review and meta-analysis. Psychiatry Research 187, 441-453.

Chiesa, A., Serretti, A. \& Jakobsen, J. C. (2013). Mindfulness: Top-down or bottom-up emotion regulation strategy? Clinical Psychology Review 33, 82-96.

Cramer, H., Lauche, R., Paul, A. \& Dobos, G. (2012). Mindfulness-based stress reduction for breast cancer-A systematic review and meta-analysis. Current Oncology 19, e343-e352.

Cuijpers, P., de Graaf, R. \& van Dorsselaer, S. (2004). Minor depression: risk profiles, functional disability, health care use and risk of developing major depression. Journal of Affective Disorders 79, 71-9.

Cuijpers, P. \& Smit, F. (2004). Subthreshold depression as a risk indicator for major depressive disorder: A systematic review of prospective studies. Acta Psychiatrica Scandinavica 109, 325-331.

Cuijpers, P., Smit, F., Oostenbrink, J., De Graaf, R., Ten Have, M. \& Beekman, A. (2007a). Economic costs of minor depression: A population-based study. Acta Psychiatrica Scandinavica 115, 229-236.

Cuijpers, P., Smit, F. \& Van Straten, A. (2007b). Psychological treatments of subthreshold depression: A meta-analytic review. Acta Psychiatrica Scandinavica 115, 434-441.

Cuijpers, P., Van Straten, A., Smit, F., Mihalopoulos, C. \& Beekman, A. (2008). Preventing the onset of depressive disorders: A meta-analytic review of psychological interventions. American Journal of Psychiatry 165, 1272-1280.

Cuijpers, P., Van Straten, A., Warmerdam, L. \& Van Rooy, M. J. (2010). Recruiting participants for interventions to prevent the onset of depressive disorders: Possibile ways to increase participation rates. BMC Health Services Research 10, 181.

De Graaf, R., Ten Have, M., Tuithof, M. \& Van Dorsselaer, S. (2013). First-incidence of DSM-IV mood, anxiety and substance use disorders and its determinants: Results from the Netherlands Mental Health Survey and Incidence Study-2. Journal of Affective Disorders 149, 100-107.

De Graaf, R., Ten Have, M., Van Gool, C. \& Van Dorsselaer, S. (2012a). Prevalence of mental disorders and trends from 1996 to 2009. Results from the Netherlands Mental Health Survey and Incidence Study-2. Social Psychiatry and Psychiatric Epidemiology 47, 203-213. 
De Graaf, R., Tuithof, M., Van Dorsselaer, S. \& Ten Have, M. (2012b). Comparing the effects on work performance of mental and physical disorders. Social Psychiatry and Psychiatric Epidemiology 47, 1873-1883.

De Raedt, R., Baert, S., Demeyer, I., Goeleven, E., Raes, A., Visser, A., . . Speckens, A. (2012). Changes in attentional processing of emotional information following mindfulness-based cognitive therapy in people with a history of depression: Towards an open attention for all emotional experiences. Cognitive Therapy and Research 36, 612-620.

Dowd, H., Hogan, M. J., McGuire, B. E., Davis, M. C., Sarma, K. M., Fish, R. A. \& Zautra, A. J. (2015). Comparison of an online mindfulness-based cognitive therapy intervention with online pain management psychoeducation: A randomized controlled study. Clinical Journal of Pain 31, 517-527.

Farb, N. A. S., Anderson, A. K. \& Segal, Z. V. (2012). The mindful brain and emotion regulation in mood disorders. Canadian Journal of Psychiatry 57, 70-77.

Ferrari, A. J., Charlson, F. J., Norman, R. E., Patten, S. B., Freedman, G., Murray, C. J. L., ... Whiteford, H. A. (2013). Burden of Depressive Disorders by Country, Sex, Age, and Year: Findings from the Global Burden of Disease Study 2010. PLoS Medicine 10, e1001547.

Fledderus, M., Bohlmeijer, E. T., Pieterse, M. E. \& Schreurs, K. M. G. (2012). Acceptance and commitment therapy as guided self-help for psychological distress and positive mental health: A randomized controlled trial. Psychological Medicine 42, 485-495.

Fledderus, M., Bohlmeijer, E. T., Smit, F. \& Westerhof, G. J. (2010). Mental health promotion as a new goal in public mental health care: A randomized controlled trial of an intervention enhancing psychological flexibility. American Journal of Public Health 100, 2372-2378.

Geschwind, N., Peeters, F., Huibers, M., Van Os, J. \& Wichers, M. (2012). Efficacy of mindfulnessbased cognitive therapy in relation to prior history of depression: Randomised controlled trial. British Journal of Psychiatry 201, 320-325.

Gratz, K. L. \& Roemer, L. (2004). Multidimensional Assessment of Emotion Regulation and Dysregulation: Development, Factor Structure, and Initial Validation of the Difficulties in Emotion Regulation Scale. Journal of Psychopathology and Behavioral Assessment 26, 41-54.

Griffiths, K. M., Farrer, L. \& Christensen, H. (2010). The efficacy of internet interventions for depression and anxiety disorders: A review of randomised controlled trials. Medical Journal of Australia 192, $\mathrm{S}_{4}-\mathrm{S}_{11}$.

Hacker, T., Stone, P. \& Macbeth, A. (2016). Acceptance and commitment therapy-Do we know enough? Cumulative and sequential meta-analyses of randomized controlled trials. Journal of Affective Disorders 190, 551-565.

Hargus, E., Crane, C., Barnhofer, T. \& Williams, J. M. G. (2010). Effects of Mindfulness on MetaAwareness and Specificity of Describing Prodromal Symptoms in Suicidal Depression. Emotion $10,34-42$.

Hayes, S. C. (2004). Acceptance and commitment therapy, relational frame theory, and the third wave of behavioral and cognitive therapies. Behavior Therapy 35, 639-665.

Hayes, S. C., Barnes-Holmes, D. \& Roche, B. (2001). Relational frame theory: A post-skinnerian account of human language and cognition. Plenum Press: New York.

Hayes, S. C., Follette, V. M. \& Linehan, M. M. (2004). Mindfulness and Acceptance: expanding the cognitivebehavioral tradition. The Guilford Press: New York.

Hayes, S. C., Luoma, J. B., Bond, F. W., Masuda, A. \& Lillis, J. (2006). Acceptance and Commitment Therapy: Model, processes and outcomes. Behaviour Research and Therapy 44, 1-25.

Hayes, S. C., Strosahl, K. \& Wilson, K. G. (1999). Acceptance \& Commitent Therapy: An experiential approach to behavior change. Guilford Press: New York.

Hayes, S. C., Strosahl, K. D. \& Wilson, K. G. (2012). Acceptance and Commitment Therapy: the Process and Practice of Mindful Change. Guilford Press: New York.

Hesser, H., Westin, V. Z. \& Andersson, G. (2014). Acceptance as a mediator in internet-delivered acceptance and commitment therapy and cognitive behavior therapy for tinnitus. Journal of Behavioral Medicine 37, 756-767. 
Hofmann, S. G., Sawyer, A. T., Fang, A. \& Asnaani, A. (2012). Emotion dysregulation model of mood and anxiety disorders. Depression and Anxiety 29, 409-416.

Hofmann, S. G., Sawyer, A. T., Witt, A. A. \& Oh, D. (2010). The Effect of Mindfulness-Based Therapy on Anxiety and Depression: A Meta-Analytic Review. Journal of Consulting and Clinical Psychology $78,169-183$.

Hölzel, B. K., Lazar, S. W., Gard, T., Schuman-Olivier, Z., Vago, D. R. \& Ott, U. (2011). How does mindfulness meditation work? Proposing mechanisms of action from a conceptual and neural perspective. Perspectives on Psychological Science 6, 537-559.

Jané-Llopis, E., Barry, M., Hosman, C. \& Patel, V. (2005). Mental health promotion works: a review. Promotion \& education Suppl 2, 9-25, 61, 67 .

Judd, L. L. (1997). The clinical course of unipolar major depressive disorders. Archives of General Psychiatry 54, 989-991.

Kabat-Zinn, J. (1982). An outpatient program in behavioral medicine for chronic pain patients based on the practice of mindfulness meditation: Theoretical considerations and preliminary results. General Hospital Psychiatry 4, 33-47.

Kabat-Zinn, J. (1990). Full catastrophe living: Using the wisdom of your body and mind to face stress, pain and illness. Delacorte: New York.

Kashdan, T. B. \& Rottenberg, J. (2010). Psychological flexibility as a fundamental aspect of health. Clinical Psychology Review 30, 865-878.

Kelders, S. M., Kok, R. N., Ossebaard, H. C. \& Van Gemert-Pijnen, J. E. W. C. (2012). Persuasive system design does matter: A systematic review of adherence to web-based interventions. Journal of Medical Internet Research 14, e152.

Kelders, S. M., Pots, W. T., Oskam, M. J., Bohlmeijer, E. T. \& Van Gemert-Pijnen, J. E. (2013). Development of a web-based intervention for the indicated prevention of depression. BMC Medical Informatics and Decision Making 13, 1-11.

Keller, M. B. \& Boland, R. J. (1998). Implications of failing to achieve successful long-term maintenance treatment of recurrent unipolar major depression. Biological Psychiatry 44, 348-360.

Keng, S. L., Smoski, M. J. \& Robins, C. J. (2011). Effects of mindfulness on psychological health: A review of empirical studies. Clinical Psychology Review 31, 1041-1056.

Kessing, L. V., Hansen, M. G. \& Andersen, P. K. (2004). Course of illness in depressive and bipolar disorders: Naturalistic study, 1994-1999. British Journal of Psychiatry 185, 372-377.

Keyes, C. L. M. (2005). Mental illness and/or mental health? Investigating axioms of the complete state model of health. Journal of Consulting and Clinical Psychology 73, 539-548.

Keyes, C. L. M., Dhingra, S. S. \& Simoes, E. J. (2010). Change in level of positive mental health as a predictor of future risk of mental Illness. American Journal of Public Health 100, 2366-2371.

Keyes, C. L. M., Wissing, M., Potgieter, J. P., Temane, M., Kruger, A. \& van Rooy, S. (2008). Evaluation of the mental health continuum-short form (MHC-SF) in Setswana-speaking South Africans. Clinical Psychology and Psychotherapy 15, 181-192.

Khoury, B., Lecomte, T., Fortin, G., Masse, M., Therien, P., Bouchard, V., ... Hofmann, S. G. (2013). Mindfulness-based therapy: A comprehensive meta-analysis. Clinical Psychology Review 33, 763-771.

Khoury, B., Sharma, M., Rush, S. E. \& Fournier, C. (2015). Mindfulness-based stress reduction for healthy individuals: A meta-analysis. Journal of Psychosomatic Research 78, 519-528.

Klainin-Yobas, P., Cho, M. A. A. \& Creedy, D. (2012). Efficacy of mindfulness-based interventions on depressive symptoms among people with mental disorders: A meta-analysis. International Journal of Nursing Studies 49, 109-121.

Kuyken, W., Warren, F. C., Taylor, R. S., Whalley, B., Crane, C., Bondolfi, G., ... Dalgleish, T. (2016). Efficacy of Mindfulness-Based Cognitive Therapy in Prevention of Depressive Relapse: An Individual Patient Data Meta-analysis From Randomized Trials. JAMA Psychiatry 73, 565-74.

Kuyken, W., Watkins, E., Holden, E., White, K., Taylor, R. S., Byford, S., ... Dalgleish, T. (2010). How does mindfulness-based cognitive therapy work? Behaviour Research and Therapy 48, 1105-1112. 
Lamers, S. M., Westerhof, G. J., Bohlmeijer, E. T., Ten Klooster, P. M. \& Keyes, C. L. (2011). Evaluating the psychometric properties of the Mental Health Continuum-Short Form (MHC-SF). Journal of Clinical Psychology 67, 99-110.

Lamers, S. M. A., Westerhof, G. J., Glas, C. A. W. \& Bohlmeijer, E. T. (2015). The bidirectional relation between positive mental health and psychopathology in a longitudinal representative panel study. Journal of Positive Psychology 10, 553-560.

Lappalainen, P., Granlund, A., Siltanen, S., Ahonen, S., Vitikainen, M., Tolvanen, A. \& Lappalainen, R. (2014). ACT Internet-based vs face-to-face? A randomized controlled trial of two ways to deliver Acceptance and Commitment Therapy for depressive symptoms: An 18-month follow-up. Behaviour Research and Therapy 61, 43-54.

Lappalainen, P., Langrial, S., Oinas-Kukkonen, H., Tolvanen, A. \& Lappalainen, R. (2015). Web-Based Acceptance and Commitment Therapy for Depressive Symptoms With Minimal Support: A Randomized Controlled Trial. Behavior Modification 39, 805-834.

Lau, M. A., Segal, Z. V. \& Williams, J. M. G. (2004). Teasdale's differential activation hypothesis: Implications for mechanisms of depressive relapse and suicidal behaviour. Behaviour Research and Therapy 42, 1001-1017.

Lauche, R., Cramer, H., Dobos, G., Langhorst, J. \& Schmidt, S. (2013). A systematic review and meta-analysis of mindfulness-based stress reduction for the fibromyalgia syndrome. Journal of Psychosomatic Research 75, 500-510.

Ledesma, D. \& Kumano, H. (2009). Mindfulness-based stress reduction and cancer: A meta-analysis. Psycho-Oncology 18, 571-579.

Levin, M. E., Pistorello, J., Seeley, J. R. \& Hayes, S. C. (2014). Feasibility of a prototype web-based acceptance and commitment therapy prevention program for college students. Journal of American College Health 62, 20-30.

Linehan, M. (1993). Cognitive-behavioral treatment of borderline personality disorder. Guilford Press: New York.

Ly, K. H., Asplund, K. \& Andersson, G. (2014a). Stress management for middle managers via an acceptance and commitment-based smartphone application: A randomized controlled trial. Internet Interventions 1, 95-101.

Ly, K. H., Trüschel, A., Jarl, L., Magnusson, S., Windahl, T., Johansson, R., . . Andersson, G. (2014b). Behavioural activation versus mindfulness-based guided self-help treatment administered through a smartphone application: A randomised controlled trial. BMJ Open 4, eoo 3440.

Ma, S. H. \& Teasdale, J. D. (2004). Mindfulness-Based Cognitive Therapy for Depression: Replication and Exploration of Differential Relapse Prevention Effects. Journal of Consulting and Clinical Psychology 72, 31-40.

Marks, I. \& Cavanagh, K. (2009). Computer-aided psychological treatments: Evolving issues. In Annual Review of Clinical Psychology, pp. 121-141.

Marks, I. M., Cavanagh, K. \& Gega, L. (2007). Computer-aided psychotherapy: Revolution or bubble? British Journal of Psychiatry 191, 471-473.

Mathers, C. D. \& Loncar, D. (2006). Projections of global mortality and burden of disease from 2002 to 2030 . PLoS Medicine 3, 2011-2030.

McCarney, R. W., Schulz, J. \& Grey, A. R. (2012). Effectiveness of mindfulness-based therapies in reducing symptoms of depression: A meta-analysis. European Journal of Psychotherapy and Counselling 14, 279-299.

Mueller, T. I., Leon, A. C., Keller, M. B., Solomon, D. A., Endicott, J., Coryell, W., ... Maser, J. D. (1999). Recurrence after recovery from major depressive disorder during 15 years of observational follow-up. American Journal of Psychiatry 156, 1000-6.

Muñoz, R. F., Cuijpers, P., Smit, F., Barrera, A. Z. \& Leykin, Y. (2010). Prevention of major depression. Annual Review of Clinical Psychology 6, 181-212.

Murray, C. J. L., Barber, R. M., Foreman, K. J., Ozgoren, A. A., Abd-Allah, F., Abera, S. F., ... Vos, T. (2015). Global, regional, and national disability-adjusted life years (DALYs) for 306 diseases 
and injuries and healthy life expectancy (HALE) for 188 countries, 1990-2013: quantifying the epidemiological transition. The Lancet 386, 2145-2191.

Nationaal Kompas Volksgezondheid (2016). https://www.volksgezondheidenzorg.info.

Nolen-Hoeksema, S. (2000). The role of rumination in depressive disorders and mixed anxiety/ depressive symptoms. Journal of Abnormal Psychology 109, 504-511.

Norton, P. J. \& Paulus, D. J. (2015). Toward a Unified Treatment for Emotional Disorders: Update on the Science and Practice. Behavior Therapy, in press.

Olatunji, B. O., Naragon-Gainey, K. \& Wolitzky-Taylor, K. B. (2013). Specificity of Rumination in Anxiety and Depression: A Multimodal Meta-Analysis. Clinical Psychology: Science and Practice 20, 225-257.

Öst, L. G. (2008). Efficacy of the third wave of behavioral therapies: A systematic review and meta-analysis. Behaviour Research and Therapy 46, 296-321.

Öst, L. G. (2014). The efficacy of Acceptance and Commitment Therapy: An updated systematic review and meta-analysis. Behaviour Research and Therapy 61, 105-121.

Piet, J. \& Hougaard, E. (2011). The effect of mindfulness-based cognitive therapy for prevention of relapse in recurrent major depressive disorder: A systematic review and meta-analysis. Clinical Psychology Review 31, 1032-1040.

Pots, W. T. M., Fledderus, M., Meulenbeek, P. A. M., Klooster ten, P. M., Schreurs, K. M. \& Bohlmeijer, E. T. (2015). Acceptance and commitment therapy as a web-based intervention for depressive symptoms: randomised controlled trial. British Journal of Psychiatry, 208, 69-77.

Ruiz, F. J. (2010). A review of acceptance and commitment therapy (ACT) empirical evidence: Correlational, experimental psychopathology, component and outcome studies. International Journal of Psychology and Psychological Therapy 10, 125-162.

Segal, Z. V., Williams, J. M. G. \& Teasdale, J. D. (2002). Mindfulness-based Cognitive Therapy for Depression: A New Approach to Preventing Relapse. The Guilford Press: New York.

Shafran, R., Clark, D. M., Fairburn, C. G., Arntz, A., Barlow, D. H., Ehlers, A., ... Wilson, G. T. (2009). Mind the gap: Improving the dissemination of CBT. Behaviour Research and Therapy 47, 902-909.

Smit, F., Willemse, G., Koopmanschap, M., Onrust, S., Cuijpers, P. \& Beekman, A. (2006). Cost-effectiveness of preventing depression in primary care patients: Randomised trial. British Journal of Psychiatry 188, 330-336.

Spek, V., Cuijpers, P., NykIiček, I., Riper, H., Keyzer, J. \& Pop, V. (2007). Internet-based cognitive behaviour therapy for symptoms of depression and anxiety: A meta-analysis. Psychological Medicine 37, 319-328.

Strauss, C., Cavanagh, K., Oliver, A. \& Pettman, D. (2014). Mindfulness-based interventions for people diagnosed with a current episode of an anxiety or depressive disorder: A meta-analysis of randomised controlled trials. PLoS ONE g, eg6rio.

Teasdale, J. D., Segal, Z. V., Williams, J. M. G., Ridgewaya, V. A., Soulsby, J. M. \& Lau, M. A. (2000). Prevention of relapse/recurrence in major depression by mindfulness-based cognitive therapy. Journal of Consulting and Clinical Psychology 68, 615-623.

Trompetter, H. R., Bohlmeijer, E. T., Veehof, M. M. \& Schreurs, K. M. G. (2014). Internet-based guided self-help intervention for chronic pain based on Acceptance and Commitment Therapy: A randomized controlled trial. Journal of Behavioral Medicine 38, 66-80.

van den Akker, M., Schuurman, A. G., Ensinck, K. T. J. L. \& Buntinx, F. (2003). Depression as a risk factor for total mortality in the community: A meta-analysis. Archives of Public Health 61, 313-332.

Veehof, M. M., Oskam, M. J., Schreurs, K. M. G. \& Bohlmeijer, E. T. (2011). Acceptance-based interventions for the treatment of chronic pain: A systematic review and meta-analysis. Pain 152, 533-542.

Vella-Brodrick, D. A. (2013). Positive psychology interventions: Research evidence, practical utility, and future steps. In Mental Well-Being: International Contributions to the Study of Positive Mental Health, pp. 331-353.

Verwey, G. \& Houben-van Herten, M. (2013). Bevolkingstrends 2013. Depressiviteit en antidepressiva in Nederland. Centraal Bureau voor de Statistiek: Den Haag/Heerlen. 
Vøllestad, J., Nielsen, M. B. \& Nielsen, G. H. (2012). Mindfulness- and acceptance-based interventions for anxiety disorders: A systematic review and meta-analysis. British Journal of Clinical Psychology 51, 239-260.

Wahbeh, H., Svalina, M. N. \& Oken, B. S. (2014). Group, One-on-One, or Internet? Preferences for Mindfulness Meditation Delivery Format and their Predictors. Open Medicine Journal 1, 66-74.

Whiteford, H. A., Ferrari, A. J., Degenhardt, L., Feigin, V. \& Vos, T. (2015). The global burden of mental, neurological and substance use disorders: An analysis from the global burden of disease study 2010. PLOS ONE 10, eo116820.

Williams, J. M. G. (2010). Mindfulness and Psychological Process. Emotion 10, 1-7.

Williams, J. M. G., Crane, C., Barnhofer, T., Brennan, K., Duggan, D. S., Fennell, M. J. V., . . Russell, I. T. (2014). Mindfulness-based cognitive therapy for preventing relapse in recurrent depression: A randomized dismantling trial. Journal of Consulting and Clinical Psychology 82, 275-286.

Wood, A. M. \& Joseph, S. (2010). The absence of positive psychological (eudemonic) well-being as a risk factor for depression: A ten year cohort study. Journal of Affective Disorders 122, 213-217.

World Health Organization (2005). Promoting mental health: Concepts, emerging evidence, practice. WHO: Geneva.

Zainal, N. Z., Booth, S. \& Huppert, F. A. (2013). The efficacy of mindfulness-based stress reduction on mental health of breast cancer patients: A meta-analysis. Psycho-Oncology 22, 1457-1465. 
CHAPTER 1 


\section{CHAPTER 2}
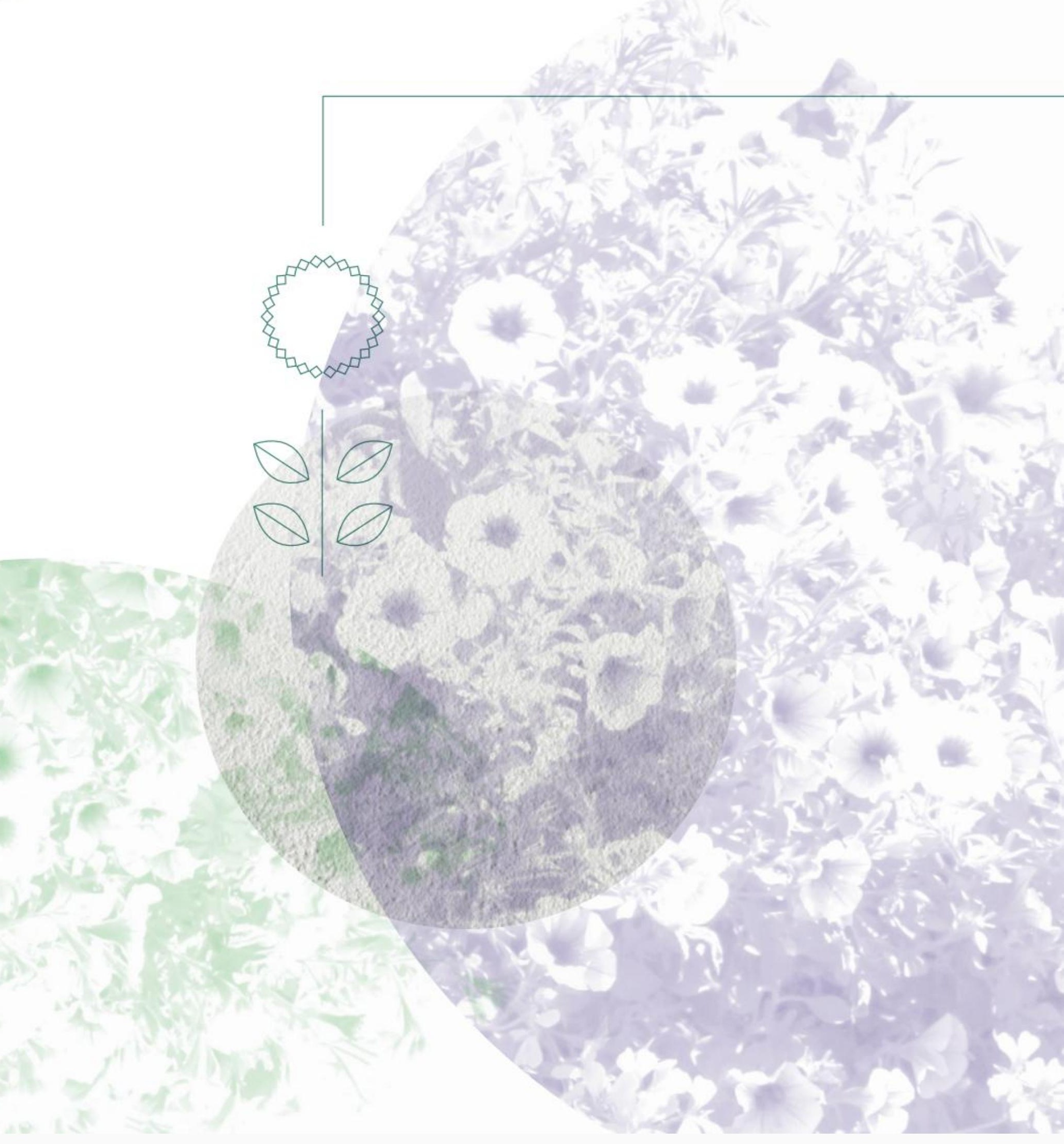


\section{The efficacy of mindfulness-based cognitive therapy as a public mental health intervention for adults with mild to moderate depressive symptomatology: a randomised controlled trial}

Pots, W.T.M., Meulenbeek, P.A.M., Veehof, M.M., Klungers, J. \& Bohlmeijer, E.T. (2014). The efficacy of mindfulness-based cognitive therapy as a public mental health intervention for adults with mild to moderate depressive symptomatology: a randomised controlled trial. PLOS ONE 9, e109789
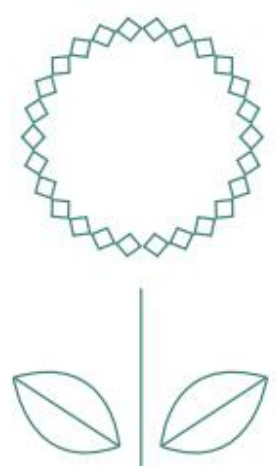


\section{Abstract}

Although there has been a growing evidence for the efficacy of mindfulness-based cognitive therapy (MBCT) for different clinical populations, its effectiveness as a public mental health intervention has not been studied. The present study evaluates a community-based MBCT intervention for adults with mild to moderate depressive symptomatology in a large multi-site, pragmatic randomised controlled trial. The participants with mild to moderate depressive symptomatology were recruited from the general population and randomised to the MBCT intervention $(n=76)$ or to a waiting list control group $(n=75)$. Participants completed measures before and after the intervention. Participants in the experimental condition also completed these measures at 3-month follow-up. In the experimental condition significant reductions in depression, anxiety, and experiential avoidance, and improvements in mindfulness and emotional- and psychological mental health were found, compared to the waiting list (effect sizes Cohen's $d=0.31-0.56$ ). These effects were sustained at 3-month follow-up. The likelihood of a clinically significant change in depressive symptoms was significantly higher for the MBCT group [odds ratio (OR) 3.026, $p<$ 0.01 at post-treatment; NNT $=5.10] . \mathrm{MBCT}$ as a public mental health intervention for adults with mild to moderate depressive symptoms seems effective and applicable in a natural setting. 


\section{Introduction}

Minor depression is a highly prevalent disorder with a large negative impact on quality of life and yielding high economic costs (Cuijpers et al., 2007). Offering interventions for people with depressive symptomatology is a necessary public mental health strategy complementary to the treatment of depression in inpatient and outpatient settings (Muñoz et al., 2010). One such effective strategy is to pro-actively offer treatments for people with mild to moderate symptoms of depression (Cuijpers et al., 2012; Jané-Llopis et al., 2005). However, a public mental health intervention needs to be attractive for people who don't suffer from severe symptoms of depression yet (Cuijpers et al., 2010). Using a positive framework and promoting positive mental health as well as reducing psychological distress offers opportunities to attract more people (Fledderus et al., 2010; Keyes et al., 2010; Wood and Joseph, 2010). Mindfulness-based cognitive therapy (MBCT) may be such an intervention, as it focuses on promoting positive mental health instead of only focusing on the reduction of psychological distress. $\mathrm{MBCT}$ is an 8-week group-based training that combines meditation exercises with cognitive techniques. It was originally designed for prevention of relapse in people with recurrent depression (Segal et al., 2002). In patients with three or more previous episodes of depression, MBCT significantly reduced the risk of relapse compared to the treatment as usual (Ma and Teasdale, 2004; Piet and Hougaard, 2011; Teasdale et al., 2000). Moreover, in a recently published randomised controlled trial, Williams et al. (2014) found evidence that the number of episodes is a marker for those with greater vulnerability due to a history of childhood trauma and adversity. Strauss et al. (2014) conducted a meta-analysis on mindfulness based interventions for people diagnosed with a current episode of an anxiety or depressive disorder and found that $\mathrm{MBCT}$ can also be effective for people who are currently depressed. MBCT targets processes such as avoidance of negative emotions and engaging with maladaptive thinking and rumination, that maintain depressive symptomatology in general (Aldao and Nolen-Hoeksema, 2010; Hayes et al., 2006). Mindfulness is often referred to as intentionally paying attention to present moment experiences in a non-judgemental way (Kabat-Zinn, 1990). Awareness and acceptance of negative experiences will reduce experiential avoidance (EA; Hayes et al., 2006). EA has been defined as the unwillingness to remain in contact with experiences such as feelings, thoughts, and bodily sensations, as an attempted means of behavioural regulation (Hayes et al., 2006). Psychological flexibility is the counterpart of experiential avoidance. As EA could be seen as an important factor that maintains depressive symptomatology, MBCT may also be effective as a public mental health intervention for people with depressive symptomatology. This fits with a growing interest in adapting $\mathrm{MBCT}$ to other psychiatric disorders, such as anxiety disorders, 
and bipolar disorder, but also treatment-resistant major depressive disorder (for a recent review see Chiesa \& Serretti (2011)). Research into the potential mechanisms of action in MBCT is in its infancy. In a recent review, Chiesa and Serretti (2014) suggested that mindfulness-based interventions may enhance positive emotion regulation strategies, as well as self-compassion levels, and decrease rumination and experiential avoidance. They suggested that these changes are associated with several clinical benefits, including the reduction of stress and depression levels, as well as the enhancement of positive emotions. Recently, Kaviani et al. (2012) found that MBCT can be effective in a non-clinical population of female students in Iran. Also, Cavanagh et al. (2013) adapted MBCT as a brief online intervention and found it to be effective in a non-clinical population of students. To our knowledge, there is no research on the effectiveness of a community-based MBCT intervention for adults with depressive symptomatology. In order to modify the original MBCT as a public mental health intervention a few changes were made in time-investment. We expected a community-based MBCT with a reduced weekly time-investment to be more acceptable for the target group.

The aim of this study was to evaluate the effect of mindfulness-based cognitive therapy modified as a public mental health intervention for depressive symptomatology, in a sample of self-referred adults with mild to moderate depressive symptomatology, and offered by community mental health centres. We evaluated the effect with respect to various psychological variables, such as depression, anxiety and positive mental health. We hypothesized that changes in depressive symptoms would be mediated by psychological flexibility and mindfulness. To strengthen the trial's external validity, the intervention was studied in its natural setting.

\section{Method}

The protocol for this trial and supporting CONSORT checklist are available as supporting information; see Checklist $\mathrm{S} 1$ and Protocol S1. This study was approved by the METiGG, a medical ethics committee for research in mental health settings in The Netherlands. In addition, this study has been registered in The Nederlands Trial Register, the Primary Dutch register for clinical trials (NTR2096).

\section{Design}

A pragmatic, multi-site, randomised controlled trial was conducted comparing MBCT with a control condition. Participants were randomly assigned after receiving their written consent, either to $\mathrm{MBCT}$ or to the waiting list control, by means of a centrally conducted randomisation process executed by an independent researcher. 
The randomisation was carried out for the two groups with stratification on gender, using a computer generated random sequence of numbers. The control condition consisted of a waiting list, where wait-listed people were free to use other kinds of care. The wait-listed participants knew that they could start the training after the experimental condition had completed the intervention, i.e. after 3 months. The study is pragmatic as it mimics the Dutch health care system as closely as possible in terms of patient recruitment, conducting intake, offering interventions, and monitoring outcomes.

\section{Participants and procedure}

Participants were recruited from November 2009 until October 2010, through advertisements in regional newspapers, information booklets and general practitioners. Four Dutch community mental health centres, from both urban and rural areas, participated in the study. In the advertisements, distributed within the regions of the participating community mental health services, the target group was described as adults who were hindered by depressive symptoms. Applicants were referred to a specifically developed website, where they could find detailed information about the study. When interested, they were sent an information letter and an informed consent form. For screening, the standard procedures employed by the mental health institutions were used. The community mental health centres were responsible for the procedure, and the in- and exclusion criteria were examined by experienced mental health nurses on the basis of a checklist, under supervision of a clinical psychologist. The inclusion criteria were: adults of 18 years and over, presenting depressive symptoms. Applicants were excluded if diagnosed with a current severe major depressive episode (MDE; eight or nine out of a total of nine symptoms) or when having a moderate to high suicide risk, according to the Dutch version of the Mini International Neuropsychiatric Interview (MINI) (Sheehan et al., 1998; Van Vliet and De Beurs, 2007). Other exclusion criteria were: receiving psychological or pharmacological treatment for mental complaints within the last three months, and presence of other severe mental or social problems warranting treatment or likely to interfere with participation in the group course. People meeting one of the exclusion criteria were advised to seek regular treatment.

\section{Power analysis}

A sample of 60 participants per condition at post-intervention was needed to detect an effect size of 0.50 (Cohen's d) for the primary outcome with a statistical power of $(1-b)=0.80$ in a two-tailed test $(p<0.05)$. Taking into account a drop-out rate of $20 \%$, 150 eligible participants were needed. 


\section{Measures}

Measurements were taken at baseline $\left(T_{0}\right)$, and at post-treatment after 3 months $\left(T_{1}\right)$. In order to study the stability of the effect of $\mathrm{MBCT}$, the patients in the experimental group received a follow-up measurement $\left(T_{2}\right)$ at 6 months after baseline. For the control condition, the measurement at 6 months is a post-treatment measurement. All measurements had good psychometric properties and are frequently applied in international studies.

The primary outcome measure was depressive symptomatology, measured by the Dutch version of the Center of Epidemiological Studies - Depression Scale (CES-D; 20 items, score o-6o) (Radloff, 1977). Higher scores mean more depressive symptoms (Haringsma et al., 2004; Radloff, 1977).

Secondary outcome measures were anxiety symptoms and positive mental health. Anxiety was measured by the Hospital Anxiety and Depression Scale - Anxiety subscale (HADS-A; 7 items, score 0-21) (Zigmond and Snaith, 1983) for assessing the presence and severity of anxiety symptoms. Higher scores mean more anxiety symptoms (Spinhoven et al., 1997; Zigmond and Snaith, 1983). Positive mental health was measured by the Mental Health Continuum - Short Form (MHC-SF; Keyes et al., 2008), that measures emotional well-being ( 3 items; score 0-15), social well-being ( 5 items; score 0-25) and psychological well-being (6 items; score 0-30). Higher scores indicate greater emotional, social, and psychological well-being (Keyes et al., 2008; Lamers et al., 2011).

Measures of proposed processes of change included measures of EA and mindfulness. The Acceptance and Action Questionnaire-II (AAQ-II; 10 items, score 10-70) (Bond et al., 2011) was used to measure the willingness to be in contact with aversive internal experiences, to accept these events, and to pursue values in the presence of the experiences. Higher scores indicate lower levels of EA or higher levels of psychological flexibility (Bond et al., 2011; Fledderus et al., 2012). The Five Facet Mindfulness Questionnaire (FFMQ; Baer et al., 2006) was used to measure mindfulness in five sub-dimensions: 1 ) observing ( 8 items), defined in terms of noticing or attending to internal and external experiences; 2 ) describing ( 8 items), defined in terms of labelling internal experiences with words; 3 ) acting with awareness ( 8 items), defined in terms of attending to one's activities of the moment (opposite of acting on automatic pilot); 4) non-judging of inner experience ( 8 items), defined in terms of taking a non-evaluative stance toward thoughts and feelings; and 5) and non-reactivity to inner experience ( 7 items), defined in terms of allowing thoughts and feelings to come and go, without getting caught up in or carried away by them. Facet scores range from 8 to 40 (except for the non-reactivity, which ranges from 7 to 35), with higher scores indicating more mindfulness (Baer et al., 2006; Bohlmeijer et al., 2011). 
To evaluate the level of satisfaction of the participants after the intervention, a self-developed evaluation questionnaire was used, including a question on how the participants evaluated the program using a scale from 1 (very poor) to 10 (excellent).

\section{Waiting list control}

Participants in the control condition participated in the MBCT training after a 3-month waiting list period. Participants were instructed to seek help from their general practitioner, family or other sources, as they normally would, should they encounter symptomatic deterioration or other difficulties during the waiting list period.

\section{Intervention}

In this study MBCT was delivered according to the guidelines of Segal et al. (2002). The original training was adapted to suit a public mental health approach. The intervention was aimed at people with mild to moderate symptoms of depression. In order to lower the threshold for people without severe distress to participate in MBCT, the participants were asked to practice meditations for 15 minutes a day instead of the original 45 minutes a day, and the sessions were limited to 1,5 hours instead of the original 2,5 hours. To ensure that all of the elements of the original course were preserved, the eight-session training was extended to 11 sessions. Key themes of the sessions included awareness (sessions 1, 2, 3), acceptance (sessions 5, 7, 9, 10) and disengaging from thoughts (sessions $4,6,8$ ), with the last session (session 11) focusing on evaluation and integration. The training teaches skills to become more aware of, and to relate differently to thoughts, feelings and bodily sensations. A core feature of the training is to learn to become aware of, and disengage from habitual dysfunctional (cognitive) routines, to stop reacting automatically to internal experiences, and to act more 'mindfully'. During the period of the training, the program consisted of daily homework exercises. The exercises were aimed at increasing attention to present moment experiences in a non-judgmental way, together with exercises designed to integrate application of awareness skills into daily life. To support homework assignments, participants received weekly homework registration forms, guided (taped) and unguided meditations, and information in a booklet. Group sizes varied between eight and 15 participants.

\section{Therapists}

The MBCT instructors were all experienced psychologists and mental health nurses, with extensive former training in the original MBCT protocol by Segal (2002) and Group psychotherapy. The trainers were also experienced meditators, with meditation experience ranging from 2 to 15 years. 


\section{Statistical analyses}

The statistical analyses were performed using SPSS 18 . The data was analysed on an intention-to-treat (ITT) basis. Missing values at baseline, post-intervention and follow-up were imputed with the use of SPSS Missing Value Analysis on the continuous measures with the expectation-maximization (EM) method. This method computes missing values based on maximum likelihood estimates using observed data in an iterative process (Dempster et al., 1977). The total percentage of missing data $\left(T_{0}-T_{1}-T_{2}\right)$ was $5 \%$, due to unanswered items $(0,6 \%)$ and incomplete assessments $(4,4 \%)$. A comparison of results based on the imputed intention-to-treat sample versus the observed data revealed similar outcomes. Therefore, only the results from the intention-to-treat analyses are reported.

Independent sample t-tests and chi-square tests were conducted to examine differences between the two groups at baseline on sociodemographic variables and outcome measures. Analysis of covariance (ANCOVA) was conducted with depressive symptoms post-treatment as the dependent variable, treatment group as the independent variable, and five covariates consisting of pre-treatment depression score and four dummy-coded variables for each treatment site. The same analyses were conducted for the secondary measures. Analysis of variance (ANOVA) was conducted with process measures post-treatment as the dependent variable and treatment group as the independent variable. Assumptions for performing parametric analysis of (co)variance were all met. To investigate whether the effects in the intervention condition were maintained at follow-up paired-sample t-tests were carried out, comparing the scores on the follow-up $\left(T_{2}\right)$ with those at baseline $\left(T_{0}\right)$. Also, paired-sample t-tests were carried out for the control group, to measure the effect of the MBCT intervention after the waiting time period, comparing scores at post-treatment ( $T_{2}$ for the control group) with those at pre-treatment ( $T_{1}$ for the control group).

Effect sizes at post-treatment $\left(T_{0}-T_{1}\right)$ were calculated with Cohen's $d$ using the means and the pooled standard deviations of the measurements in the conditions. For the effect sizes at follow-up $\left(T_{0}-T_{2}\right)$ the Cohen's $d$ was corrected for dependence among means by using the correlation between the two means (Morris and DeShon, 2002). To interpret Cohen's $d$ an effect size of less than 0.33 is considered small, while 0.33 to 0.55 is considered moderate and effect sizes of 0.56 to 1.2 are considered large (Lipsey and Wilson, 1993). Comparisons were two-tailed and interpreted with a significance value of $\mathrm{p}<0.05$.

With the Jacobson and Truax methodology, the proportion of participants was determined who made a clinically significant change on the CES-D from baseline to post-treatment (Jacobson and Truax, 1991). First, the reliable change was calculated with the reliable change index (RCI). Jacobson and Truax suggest that subjects can 
be considered to have improved when they shift from a dysfunctional distribution to a functional one, and the reliable change scores exceed measurement error (calculated by dividing the difference between the pretest and posttest scores by the standard error of the measurement). Second, the recovery criterion was defined as a post-treatment score below the cut-off value of 16 for clinically relevant depressive symptoms (Beekman et al., 1997; Smit et al., 2006). Because we studied a population with a mild to moderate symptomatology, the mean score at baseline was already at the recovery criterion $(M=16.04$, range $1-43$, sd $=8.08)$. A clinically significant change on the CES-D is thus defined as having a reliable change between the measurements, which required a post-treatment score below the cut-off of 16 . Participants that had a clinically significant change were either coded 1 (implying a favourable treatment response, 'success') or o ('failure'). The binary outcome was used to calculate the odds ratio (OR) using logistic regression. Based on the clinically significant change proportions, the number needed to treat (NNT) was calculated (Cook and Sackett, 1995). To provide a more complete representation of the effects of the intervention, the outcomes were also analysed for intervention completers only (somewhat arbitrarily defined as participants that attended at least 9 sessions).

The process measures were expected to be mediators between the MBCT intervention and post-treatment levels of depressive symptomatology (CES-D). Mediation was performed for all process measures that were significantly different between the intervention and control condition in the ANOVA. Then, all steps outlined by Baron and Kenny (1986) were used. In the first step linear regression analysis was performed, with treatment group as independent variable and depressive symptoms post-treatment as the dependent variable. In the second step we tested the effect of the independent variable on the proposed mediators with linear regression analyses, with treatment group as independent variable and the residual change scores of the proposed mediators as the dependent variables. The third step tested the effect of the proposed mediator on the dependent variable. The indirect effect of the mediator on the outcome was assessed to examine whether an increase in psychological flexibility and mindfulness during the intervention would mediate the effects of the intervention on depressive symptomatology at post-intervention. Simple mediational analyses with bootstrapping procedures ( $n=5000$ bootstrap resamples) were used to assess the indirect effect of the mediator on the outcome (Preacher and Hayes, 2008). An indirect effect was considered significant in the case zero was not contained in the $95 \%$ confidence interval. 


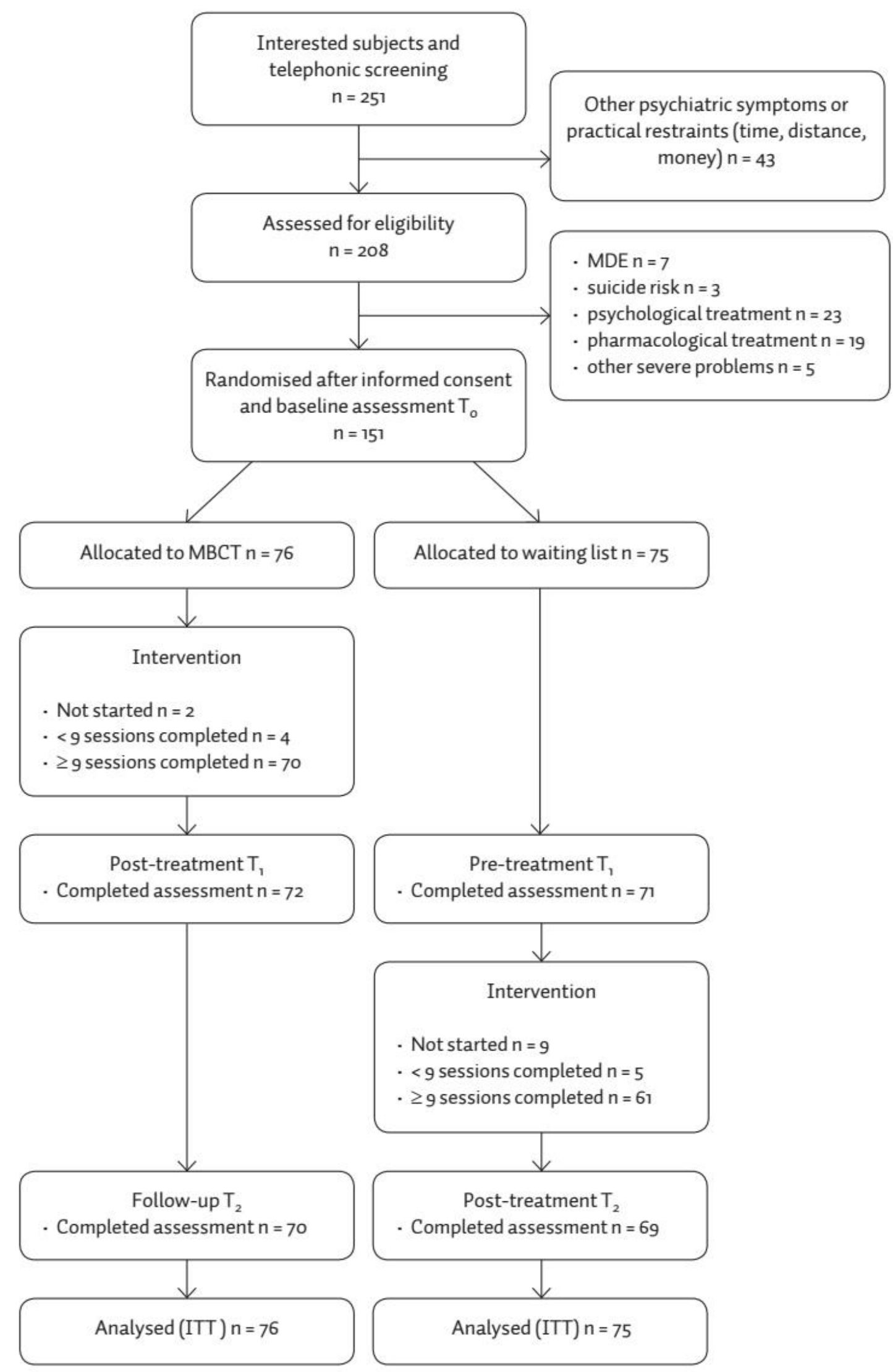

Figure 1 - Participant flow.

Note. $\mathrm{MDE}=$ Major depressive episode. 


\section{Results}

\section{Enrolment, treatment adherence, satisfaction and drop-out}

Figure 1 provides an overview of the flow of participants. A total of 251 persons were interested in the training. During telephone screening, 43 persons presented other psychiatric symptoms or practical restraints that precluded them from participation in the trial. The remaining 208 applicants were assessed for eligibility. Through interviewing, a further 57 were excluded. After signing the informed consent form, the included 151 participants were randomly assigned to the MBCT intervention ( $n=$ 76) and the waiting list condition $(n=75)$.

After randomisation, two participants decided not to start with the intervention due to time constraints $(n=1)$ and health problems $(n=1)$. Four other participants in the MBCT group ( $8 \%$ ) did not complete the intervention (attendance of at least 9 sessions). The reasons given for non-completion were that the intervention was too time consuming $(n=1)$, practicality reasons $(n=1)$, insufficient concentration $(n=1)$, and an unknown reason $(n=1)$. Two of the four community mental health centres evaluated the course resulting in anonymous evaluation forms of all participants, including those who did not participate in the study. Based on these results the intervention was evaluated as positive on a scale from 1 to $10(\mathrm{~m}=7.98, \mathrm{sd}=0.72$, $n=130)$.

At $T_{1}$ (post-treatment for the MBCT group and pre-treatment for the waiting list group), data was available for 143 participants (drop-out rate 5.3\%) and at $\mathrm{T}_{2}$ (follow-up for the MBCT group and post-treatment for the waiting list group), data was available for 139 participants (drop-out rate $7.9 \%$ ). There were no significant differences at baseline on all the measurements between participants who completed the assessments and those who did not complete all measures.

\section{Baseline characteristics}

Table 1 shows an overview of the participants' characteristics. Participants had a mean age of 48 years $(\mathrm{sd}=11.29$, range $20-81$ ) and were predominantly female $(78.1 \%)$. The majority was of Dutch origin ( $96 \%)$, had a paid job $(66.9 \%)$, and were living with a partner (75.5\%). The level of education was high for $65.5 \%$ of the participants and intermediate for $30.5 \%$. At the entry of the study, $2.6 \%$ met the criteria of mild MDE and $4 \%$ were diagnosed with moderate MDE. There were no significant differences at baseline between the MBCT group and the control group for any of the demographic variables or outcome measurements. Also, there were no significant changes in the waiting time period for the control group, comparing scores at baseline $\left(T_{0}\right)$ with pre-treatment measurement $\left(T_{1}\right)$, on any of the outcome measures. 
Table 1 - Baseline characteristics of the total sample, MBCT intervention and waiting list control condition.

\begin{tabular}{|c|c|c|c|c|c|c|c|}
\hline & & МBCT & $=76)$ & Waiting & st $(n=75)$ & Total (n & 151) \\
\hline & & $\mathbf{n}$ & $\%$ & $\mathbf{n}$ & $\%$ & $\mathbf{n}$ & $\%$ \\
\hline \multirow[t]{2}{*}{$\mathrm{Age}^{\mathrm{a}}$} & Mean (sd) & $\begin{array}{l}47.99 \\
(10.59)\end{array}$ & & $\begin{array}{l}47.88 \\
(12.02)\end{array}$ & & $\begin{array}{l}47.93 \\
(11.29)\end{array}$ & \\
\hline & Range & $24-74$ & & $20-81$ & & $20-81$ & \\
\hline \multirow[t]{2}{*}{ Gender $^{b}$} & Female & 59 & 77,6 & 59 & 78,7 & 118 & 78,1 \\
\hline & Male & 17 & 22,4 & 16 & 21,3 & 33 & 21,9 \\
\hline \multirow[t]{2}{*}{ Marital status ${ }^{b}$} & Single & 17 & 22,4 & 20 & 26,7 & 37 & 24,5 \\
\hline & Living with partner & 59 & 77,6 & 55 & 73,3 & 114 & 75,5 \\
\hline \multirow[t]{2}{*}{ Race $^{b}$} & Dutch & 71 & 93,4 & 74 & 98,7 & 145 & 96,0 \\
\hline & Other & 5 & 6,6 & 1 & 1,3 & 6 & 4,0 \\
\hline \multirow[t]{3}{*}{ Education $^{\text {b }}$} & Low & 2 & 2,6 & 4 & 5,3 & 6 & 4,0 \\
\hline & Middle & 21 & 27,6 & 25 & 33,3 & 46 & 30,5 \\
\hline & High & 53 & 69,7 & 46 & 61,3 & 99 & 65,5 \\
\hline \multirow[t]{2}{*}{ Employment $^{\mathrm{b}}$} & Paid & 53 & 69,7 & 48 & 64,0 & 101 & 66,9 \\
\hline & No paid & 23 & 30,3 & 27 & 36,0 & 50 & 33,1 \\
\hline \multirow[t]{2}{*}{ Diagnosis $^{b}$} & Mild MDE & 3 & 3,9 & 1 & 1,3 & 4 & 2,6 \\
\hline & Moderate MDE & 0 & 0,0 & 6 & 8,0 & 6 & 4,0 \\
\hline
\end{tabular}

Note. MDE, major depressive episode.

${ }^{a}$ No significant differences between intervention and control condition ( $t$-test with $p<0.05$ ).

${ }^{b}$ No significant differences between intervention and control condition (chi-square-test with $\mathrm{p}<0.05$ ).

\section{Treatment effects}

\section{Primary outcome}

The means and standard deviations for the primary outcome measure, the results of the ANCOVA and the effect sizes are presented in Table 2. Compared to the control condition, participants in the intervention condition reported significantly decreased depressive symptoms at post-treatment (Table 2). The effects of the intervention condition on depressive symptoms were maintained at follow-up compared to baseline measurement $[t(75)=-3.46, p<0.01]$. Moderate effect sizes were found at post-treatment $(d=0.50)$ and follow-up $(d=0.40)$. The control group also showed significant reductions in depression after they received the intervention at $\mathrm{T}_{2}[\mathrm{t}(74)=$ $-3.03, p<0.01]$. 
Table 2 - Means and standard deviations for outcome measures, results of ANCOVA for intervention effects, and Cohen's d.

\begin{tabular}{|c|c|c|c|c|c|c|c|}
\hline \multirow[b]{2}{*}{ Measures } & \multicolumn{2}{|c|}{$\begin{array}{l}\text { MBCT } \\
(n=76)\end{array}$} & \multicolumn{2}{|c|}{$\begin{array}{l}\text { Waiting list } \\
(n=75)\end{array}$} & \multirow[b]{2}{*}{$F(d f)^{b}$} & \multirow[b]{2}{*}{$\mathbf{p}$} & \multirow[b]{2}{*}{ d } \\
\hline & $M^{a}$ & $S^{a}$ & $M^{a}$ & $S^{a}$ & & & \\
\hline \multicolumn{8}{|l|}{ Primary outcome } \\
\hline CES-D baseline & 15.62 & 7.86 & 16.46 & 8.32 & & & \\
\hline CES-D post-treatment & 11.79 & 8.76 & 16.43 & 9.94 & $10.91(1)$ & 0.001 & 0.50 \\
\hline CES-D follow-up & 11.77 & 8.25 & 13.60 & 8.88 & & & \\
\hline \multicolumn{8}{|l|}{ Secondary outcomes } \\
\hline HADS-A baseline & 7.96 & 3.40 & 8.57 & 3.58 & & & \\
\hline HADS-A post-treatment & 6.14 & 3.52 & 8.22 & 3.89 & $12.42(1)$ & 0.001 & 0.56 \\
\hline HADS-A follow-upc & 4.88 & 2.88 & 6.54 & 3.84 & & & \\
\hline MHC-SF-EM baseline & 3.15 & 0.95 & 3.15 & 0.78 & & & \\
\hline MHC-SF-EM post-treatment & 3.46 & 0.85 & 3.19 & 0.88 & $5 \cdot 32(1)$ & 0.023 & 0.31 \\
\hline MHC-SF-EM follow-up ${ }^{c}$ & 3.54 & 0.95 & 3.61 & 0.78 & & & \\
\hline MHC-SF-SOC baseline & 2.38 & 1.04 & 2.28 & 0.96 & & & \\
\hline MHC-SF-SOC post-treatment & 2.72 & 1.08 & 2.49 & 1.05 & $1.62(1)$ & 0.206 & 0.22 \\
\hline MHC-SF-SOC follow-up ${ }^{c}$ & 2.91 & 1.12 & 2.85 & 0.86 & & & \\
\hline MHC-SF-PSY baseline & 2.92 & 0.89 & 2.96 & 1.00 & & & \\
\hline MHC-SF-PSY post-treatment & 3.26 & 0.98 & 2.90 & 1.11 & $8.60(1)$ & 0.004 & 0.34 \\
\hline MHC-SF-PSY follow-up ${ }^{c}$ & 3.37 & 0.93 & 3.41 & 0.89 & & & \\
\hline
\end{tabular}

Note. ANCOVA, Analysis of covariance; CES-D, Center for Epidemiologic Studies - Depression scale; HADS-A, Hospital Anxiety and Depression Scale - Anxiety subscale; MHC-SF, Mental Health Continuum - Short form; EM, emotional; SOC, social; PSY, psychological.

a Unadjusted condition means and standard deviations (sd).

${ }^{b}$ F-value, corrected for baseline values.

c Follow-up for intervention group and post-treatment for waiting list group.

\section{Clinically significant change}

The reliable change on the CES-D appeared to be a pre-post difference of at least 7 scale points. Clinically significant change was thus defined as a recovery condition of a score $\leq 16$ points on the CES-D $(n=138)$ and a RCI of 7 points. The proportion of participants with a score of $\geq 7$ at $T_{0}$ that reached a clinically significant change was $24 / 70(34 \%)$ in the intervention group, versus 10/68 (15\%) in the control condition [OR 3.026, 95\% confidence interval (CI) 1.316-6.961, $p<0.01$, NNT = 5.10, under an intention-to-treat analysis]. These results compare well with completers-only findings: OR 2.916, 95\% CI 1.252-6.795, p< 0.01, NNT = 5.26. 


\section{Secondary outcome measures}

The means and standard deviations for the secondary outcome measures, the results of the ANCOVA and the effect sizes are presented in Table 2. Compared to the control condition, participants in the intervention condition reported significantly decreased anxiety symptoms after the intervention. At follow-up, the effects of the intervention condition on anxiety symptoms were maintained compared to baseline measurement $[t(75)=-8.40, p<0.001]$. The effect sizes for anxiety symptoms at post-treatment $(d=0.56)$ and at follow-up $(d=0.97)$ were large. The control group also showed significant reductions in anxiety after they received the intervention at $\mathrm{T}_{2}[\mathrm{t}(74)=$ $-5.15, p<0.001]$.

At post treatment, significant improvements in emotional well-being and psychological well-being were found. The effects of the intervention condition on emotional and psychological well-being were maintained at follow-up compared to baseline measurement [emotional well-being $\mathrm{t}(75)=4.13, \mathrm{p}<0.001$; psychological well-being $\mathrm{t}(75)=5.20, \mathrm{p}<0.001]$. Effect sizes at post-treatment were small (emotional well-being, $d=0.31$ ) to moderate (psychological well-being, $d=0.34$ ). The effect sizes at follow-up were moderate (emotional well-being, $d=0.50$ ) to large (psychological well-being, $d=0.56$ ). No significant effects were found at post-treatment for social well-being, with a small effect size $(\mathrm{d}=0.22)$. At follow-up, there was a significant increase in social well-being compared to baseline measurement $[t(75)=5.58, p<$ $0.001]$, with a large effect size $(d=0.63)$.

The control group showed significant reductions on positive mental health after they received the intervention at $T_{2}$ [social well-being $t(74)=4.79, p<0.001$; emotional well-being $\mathrm{t}(74)=5.47, \mathrm{p}<0.001$; psychological well-being $\mathrm{t}(74)=6.21$, $\mathrm{p}<0.001]$.

\section{Process measures}

Table 3 shows all process measurements, the results of the ANOVA, and the effectsizes. Compared to the control condition, participants in the intervention condition showed significant improvement in psychological flexibility and all mindfulness facets (except for FFMQ Describing). The effects of the intervention condition on all process measures were maintained at follow-up compared to baseline measurement [psychological flexibility, $\mathrm{t}(75)=6.17, \mathrm{p}<0.001$; FFMQ Observing, $\mathrm{t}(75)=7.67$, $\mathrm{p}<0.001$; FFMQ Describing, $\mathrm{t}(75)=6.82, \mathrm{p}<0.001$; FFMQ Acting with awareness, $t(75)=6.20, p<0.001$; FFMQ Non-judging of inner experience, $t(75)=6.46, p<0.001$; FFMQ Non-reactivity to inner experience, $t(75)=8.97, p<0.001]$. The effect sizes post-treatment were small to large $(d=0.13-0.84)$ and large at follow-up $(d=0.71$ 1.03). 
Table 3 - Means and standard deviations for process measures, results of ANOVA and Cohen's for intervention effects.

\begin{tabular}{|c|c|c|c|c|c|c|c|}
\hline \multirow[b]{2}{*}{ Process outcomes } & \multicolumn{2}{|l|}{$\begin{array}{l}\text { MBCT } \\
(n=76)\end{array}$} & \multicolumn{2}{|c|}{$\begin{array}{l}\text { Waiting list } \\
(n=75)\end{array}$} & \multirow[b]{2}{*}{$F(d f)$} & \multirow[b]{2}{*}{$\mathbf{p}$} & \multirow[b]{2}{*}{ d } \\
\hline & M & SD & M & SD & & & \\
\hline AAQ-II baseline & 44.28 & 8.42 & 43.03 & 7.86 & & & \\
\hline AAQ-II post-treatment & 49.59 & 8.80 & 44.36 & 10.01 & $11.62(1)$ & 0.001 & 0.56 \\
\hline AAQ-II follow-up ${ }^{a}$ & 50.93 & 9.04 & 48.91 & 8.89 & & & \\
\hline FFMQ - Observe baseline & 24.77 & 5.58 & 25.21 & 5.77 & & & \\
\hline FFMQ - Observe post-treatment & 27.98 & 5.15 & 25.28 & 6.29 & $8.34(1)$ & 0.004 & 0.47 \\
\hline FFMQ-Observe follow-up ${ }^{a}$ & 28.46 & 4.54 & 29.07 & 4.74 & & & \\
\hline FFMQ - Describe baseline & 26.39 & 6.14 & 27.43 & 6.89 & & & \\
\hline FFMQ - Describe post-treatment & 28.60 & 5.56 & 27.80 & 6.35 & $0.68(1)$ & 0.410 & 0.13 \\
\hline FFMQ - Describe follow-up ${ }^{a}$ & 29.24 & 6.03 & 29.77 & 6.76 & & & \\
\hline FFMQ - ActAware baseline & 20.92 & 5.45 & 22.61 & 5.48 & & & \\
\hline FFMQ - ActAware post-treatment & $25 \cdot 31$ & 5.53 & 22.67 & 6.05 & $7.86(1)$ & 0.006 & 0.46 \\
\hline FFMQ - ActAware follow-upa & 25.08 & 5.51 & 26.31 & $5 \cdot 30$ & & & \\
\hline FFMQ - NonJudge baseline & 23.64 & 6.04 & 22.87 & 5.48 & & & \\
\hline FFMQ - NonJudge post-treatment & 27.68 & 5.16 & 24.72 & 5.66 & $11.28(1)$ & 0.001 & 0.55 \\
\hline FFMQ - NonJudge follow-up ${ }^{a}$ & 28.16 & 5.57 & 28.05 & 5.52 & & & \\
\hline FFMQ - NonReact baseline & 19.73 & 4.29 & $19 \cdot 31$ & 4.29 & & & \\
\hline FFMQ - NonReact post-treatment & 23.86 & 4.27 & 20.24 & 4.32 & $26.82(1)$ & 0.000 & 0.84 \\
\hline FFMQ - NonReact follow-upa & 24.24 & 4.04 & 23.54 & 4.22 & & & \\
\hline
\end{tabular}

Note. AAQ-II, Acceptance and Action Questionnaire-II; ANOVA, Analysis of variance; FFMQ, Five Facet Mindfulness Questionnaire; Observe, observing; Describe, describing; ActAware, acting with awareness; NonJudge, non-judging of inner experience; NonReact, non-reactivity to inner experience.

${ }^{a}$ Follow-up for intervention group and post-treatment for waiting list group.

\section{Mediational analyses}

Figure 2 shows the results of the first three steps of mediation. The FFMQ Describing was excluded from the mediational analyses, having no significantly different effect in the ANOVA. The first step shows that the intervention condition had significantly decreased depressive symptoms at post-treatment, compared to the control condition. Step two shows that the intervention condition was significantly improved in psychological flexibility and mindfulness compared to the control condition. In step 3 , all change scores of the process measures were significantly associated with the scores on the CES-D at post-treatment. In the last step of the mediational analysis, 
following Preacher \& Hayes (2008), results showed that the effect of the intervention on depressive symptoms was mediated by all process measures. Full mediation was found for improvement of psychological flexibility (direct effect $\beta=0.145, p=0.057$; indirect effect $\beta=-0.387,95 \% \mathrm{CI} 0.89-3.45)$, and mindfulness facets Observing (direct effect $\beta=0.132, \mathrm{p}=0.106$; indirect effect $\beta=-0.308,95 \% \mathrm{CI} 0.98-3.60$ ), and Non-reactivity to inner experience (direct effect $\beta=0.135, \mathrm{p}=0.108$; indirect effect $\beta=$ $-0.275,95 \%$ CI $0.83-3.73$ ). Partial mediation was found for improvement of mindfulness facets Acting with awareness (direct effect $\beta=0.176, p=0.044$; indirect effect $\beta=-0.160,95 \% C I 0.18-2.84$ ), and Non-judging of inner experience (direct effect $\beta=$ $0.208, \mathrm{p}=0.011$; indirect effect $\beta=-0.158,95 \% \mathrm{CI} 0.06-1.74$ ).
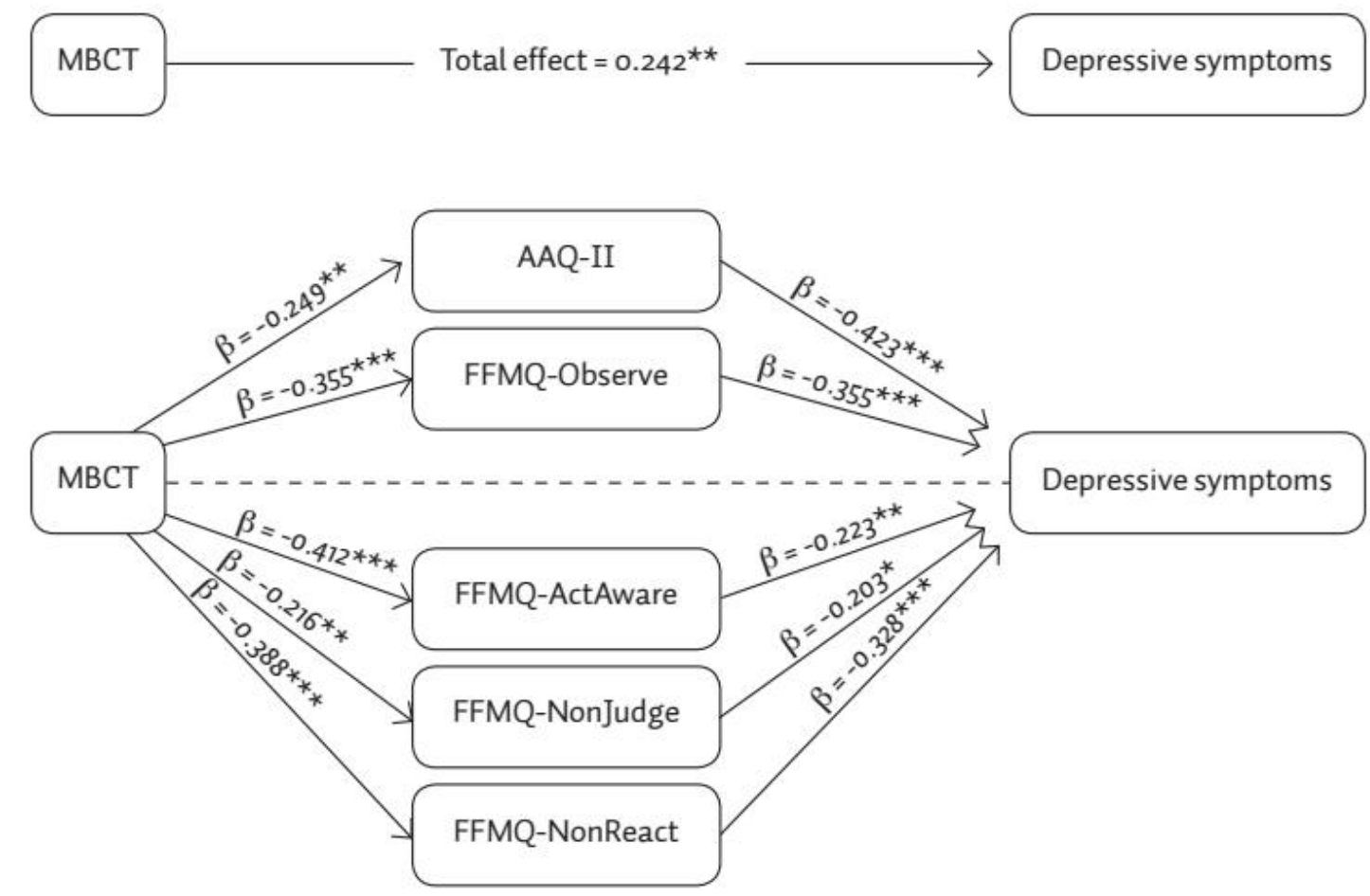

Figure 2 - Mediation model of psychological flexibility and mindfulness as mediators.

Note. AAQ-II, Acceptance and Action Questionnaire-II; FFMQ, Five Facet Mindfulness Questionnaire; Observe, observing; ActAware, acting with awareness; NonJudge, non-judging of inner experience; NonReact, non-reactivity to inner experience.

${ }^{*} \mathrm{p}<0.05 ;{ }^{* *} \mathrm{p}<0.01 ;{ }^{* \star *} \mathrm{p}<0.001$. 


\section{Discussion}

\section{Main findings}

To our best knowledge, this is the first study that evaluated MBCT as a public mental health intervention for adults with mild to moderate depressive symptomatology within a large pragmatic multi-site trial. The potential of offering MBCT to the community as a public mental health intervention is of importance, as the idea that $M B C T$ is only effective in patients suffering from recurrent depression is superseded. In the present study the participants suffered from mild to moderate depressive symptoms but differed from severe clinical samples, as indicated by the substantially lower mean degree of severity of depressive symptoms as measured by the CES-D and the MINI. This corresponds to the nature and structure of the community-based $M B C T$, offered in our study. The results from this study indicate that MBCT as a public mental health intervention is effective in reducing depressive symptoms. We found a moderate effect size for depressive symptoms at post-treatment in comparison with the control group $(d=0.50)$. This effect size is comparable to the effect size of 0.42 that was found for psychological treatments on subthreshold depression (Cuijpers and Smit, 2004). The only studies of MBCT in community-based samples to our knowledge are from Kaviani et al. (2012) and Cavanagh et al. (2013). Both conducted a randomised controlled trial in a sample of students comparing MBCT to a waiting list control group. They showed significant reductions of depressive and anxiety symptoms over time, with effect limited by low generalisability. Our study corroborates with these findings, further showing that MBCT seems to be effective in a population of adults with mild to moderate depressive symptomatology, and that $\mathrm{MBCT}$ can be used as a public mental health intervention in the community. Our finding that the results are maintained at 3 months follow-up is promising, but needs to be substantiated by longer follow-up measurements under controlled conditions. Offering a public mental health intervention in a positive framework might be less stigmatising for participants with depressive symptomatology or minor depression (Cuijpers et al., 2010). MBCT focuses on the enhancement of promoting positive skills and therefore has the potential to offer an alternative to stigmatisation.

The likelihood of a clinically significant change in depressive symptomatology in our study was substantially higher in the intervention condition compared to the waiting-list control group. As the presence of clinically relevant depressive symptoms is known to be an important risk factor for clinical depression (Cuijpers and Smit, 2004), this outcome suggests that MBCT implemented as a public mental health intervention for adults with mild to moderate depressive symptomatology may decrease the risk of developing a MDE. It is shown that the effects on clinical cases of interventions for people with sub-clinical symptomatology are most 
prominently found after longer periods of time (Cuijpers et al., 2008). However this result needs to be corroborated with longer follow-up measurement and the use of diagnostic instruments as outcome measures.

Moreover, the MBCT intervention resulted in significant reductions in anxiety symptoms with large effect sizes post-treatment and at the 3-month follow-up. The effect of MBCT on anxiety symptoms are consistent with the meta-analysis by $\bigvee \varnothing$ llestad et al. (2012), which found a large effect size $(g=0.83)$ for controlled studies of mindfulness and acceptance-based interventions for patients with anxiety symptoms. Strauss et al. (2014) found no effects on anxiety symptom severity in their meta-analysis, applying a more stringent definition and excluding trials with interventions based on Acceptance and Commitment Therapy. Our study supports the findings of $\mathrm{V} \varnothing$ llestad et al. (2012), indicating that MBCT as a public mental health intervention could be effective in not only reducing depressive symptoms, but also in significantly reducing anxiety symptoms post-treatment and, even more substantially, at the 3-month follow-up. As anxiety symptoms often coexist with depression and may precipitate depression (Horn and Wuyek, 2010), these findings could indicate that the application of MBCT has the potential to further decrease the incidence of depression. However, this needs to be substantiated by further research.

The results also show a significant effect on positive mental health. It thus seems that MBCT has the potential not only to reduce psychological distress, but also to improve emotional, psychological, and social (only at follow-up) well-being as well. This finding confirms earlier studies that show that MBCT can promote well-being in patients with anxiety or depression (Kaviani et al., 2012; Kuyken et al., 2008). The effects of MBCT on positive mental health are of importance. There is growing evidence that positive mental health and psychopathology are related but different dimensions of mental health (Lamers et al., 2012), and that positive mental health is a protective factor against mental illnesses (Keyes et al., 2010; Wood and Joseph, 2010). Several researchers suggest that aspects of psychological well-being (e.g. meaning, mastery, autonomy, goals) increase personal resilience (Fava and Tomba, 2009; Ruini and Fava, 2012; Ryff et al., 2012). The findings of this study suggest that adults with mild to moderate depressive symptomatology that participated in this public mental health intervention will be able to better cope with life adversity in the future. Moreover, offering a public mental health intervention in a positive framework might be less stigmatising for participants with depressive symptomatology (Cuijpers et al., 2010).

Mediational analyses show that the efficacy of MBCT compared to the control group on reducing post-treatment depressive symptoms is mediated by an increase in psychological flexibility and all mindfulness skills, except for the subscale 'describe'. Our results are in line with Kuyken et al. (2010), which showed that 15-month follow- 
up level of depression were mediated by mindfulness skills and self-compassion. Developing a compassionate attitude toward one's own negative thoughts and feelings mediated the effect of $\mathrm{MBCT}$ on depressive symptoms and relapse. These findings are also in line with earlier studies that demonstrated the association between low psychological flexibility (i.e. experiential avoidance) and mindfulness and psychopathology (Hayes et al., 2006; Kashdan and Rottenberg, 2010; McCarney et al., 2012). The increase of psychological flexibility and mindfulness that mediated the effects of $\mathrm{MBCT}$ on depressive symptomatology suggests that participants have gained additional adaptive emotion regulation skills in response to negative affect-producing stressors (Chiesa et al., 2014; Kashdan and Rottenberg, 2010).

The original MBCT training was designed for people with a history of depression and requires participants to commit to a 2.5-hour group session and to 45 to 60 minutes of mindfulness practice each day for 8 weeks (Segal et al., 2002). The current study suggests that mindfulness exercises of a total of 15 minutes a day may be effective for adults with mild to moderate depressive symptomatology. Much of the research on the effects of $\mathrm{MBCT}$ is conducted based on a clinical population. For the non-clinical population, mostly still working and active socially, the required time commitment on a weekly and daily basis may be a barrier to effectively integrate exercises into daily life. Carmody and Baer (2009) concluded in their review that the correlation between mean effect size and number of in-class hours was non-significant for both clinical and non-clinical samples. They suggested that adaptations that include less class time may be worthwhile for populations for whom reduction of psychological distress is an important goal and for whom longer time commitment may be a barrier to their ability or willingness to participate. Our findings are in line with a number of studies which have shown that short-term meditation can lead to more tolerance, and a lower distress of pain and perceived stress (e.g. Klatt et al., 2009; Liu et al., 2013). For example, Klatt et al. (2009) showed that a 60-minute training together with 20 minutes of daily practice of meditation can have a significant positive effect on levels of perceived stress in healthy working adults $(p=0.0025)$. Also, Cavanagh et al. (2013) effectively adapted their online MBCT to a brief intervention with daily mindfulness meditation practices of 10 minutes. Our finding that an 11-week MBCT training with 15 minutes of daily mindfulness meditation practice can sufficiently and significantly reduce depressive symptomatology seems promising from a public mental health perspective and is in line with Carmody and Baer (2009). The finding that the community-based MBCT intervention was very positively evaluated by the participants (scoring 7.98 out of 10), and that very few people dropped out of the intervention or dropped out of the study, underscores the feasibility and the attractiveness of the intervention. 


\section{Limitations}

Some limitations must also be acknowledged. First, for the design of the study as a waiting-list compared RCT, controlling for the influence of possible non-specific factors, such as attention and social interaction, was unlikely. Future research should use an active control intervention or an attention placebo controlled design to overcome this limitation. Secondly, the study used a short follow-up period of 3 months for which the follow-up was limited to a within group analysis. For ethical reasons, the time until the control group could receive the intervention was limited to 3 months. For future research, a longer follow-up (e.g. 1-year follow-up) is recommended to study the impact of MBCT on the incidence of depression. Third, the design was a pragmatic randomised controlled trial with self-referred participants, so the results of the study may have been influenced by a selection bias. All measures were self-report: no psychiatric diagnoses were available because participants were recruited from the general public. Generalisability of the findings to patients seeking treatment cannot be assumed. On the other hand, no restrictions were made to the level of depressive symptoms, as is customary to the procedures applied in community mental health centres. In this regard, the study was representative for standard general practice.

\section{Conclusion}

This study shows that MBCT as a public mental health intervention for adults with mild to moderate depressive symptomatology is effective by not only reducing depressive symptoms and anxiety symptoms, but also enhancing positive mental health and psychological flexibility. Furthermore, this study shows that the intervention is applicable and effective in a natural setting. 


\section{References}

Aldao, A. \& Nolen-Hoeksema, S. (2010). Specificity of cognitive emotion regulation strategies: A transdiagnostic examination. Behaviour Research and Therapy 48, 974-983.

Baer, R. A., Smith, G. T., Hopkins, J., Krietemeyer, J. \& Toney, L. (2006). Using self-report assessment methods to explore facets of mindfulness. Assessment 13, 27-45.

Baron, R. M. \& Kenny, D. A. (1986). The Moderator-Mediator Variable Distinction in Social Psychological Research. Conceptual, Strategic, and Statistical Considerations. Journal of Personality and Social Psychology 51, 1173-1182.

Beekman, A. T. F., Deeg, D. J. H., Van Limbeek, J., Braam, A. W., De Vries, M. Z. \& Van Tilburg, W. (1997). Criterion validity of the Center for Epidemiologic Studies Depression scale (CES-D): Results from a community-based sample of older subjects in the Netherlands. Psychological Medicine 27, 231-235.

Bohlmeijer, E., Ten Klooster, P. M., Fledderus, M., Veehof, M. \& Baer, R. (2011). Psychometric properties of the five facet mindfulness questionnaire in depressed adults and development of a short form. Assessment 18, 308-320.

Bond, F. W., Hayes, S. C., Baer, R. A., Carpenter, K. M., Guenole, N., Orcutt, H. K., ... Zettle, R. D. (2011). Preliminary Psychometric Properties of the Acceptance and Action Questionnaire-II: A Revised Measure of Psychological Inflexibility and Experiential Avoidance. Behavior Therapy $42,676-688$.

Carmody, J. \& Baer, R. A. (2009). How long does a mindfulness-based stress reduction program need to be? A review of class contact hours and effect sizes for psychological distress. Journal of Clinical Psychology 65, 627-638.

Cavanagh, K., Strauss, C., Cicconi, F., Griffiths, N., Wyper, A. \& Jones, F. (2013). A randomised controlled trial of a brief online mindfulness-based intervention. Behaviour Research and Therapy 51, 573-578.

Chiesa, A., Anselmi, R. \& Serretti, A. (2014). Psychological mechanisms of mindfulness-based interventions: What do we know? Holistic Nursing Practice 28, 124-148.

Chiesa, A. \& Serretti, A. (2011). Mindfulness based cognitive therapy for psychiatric disorders: A systematic review and meta-analysis. Psychiatry Research 187, 441-453.

Cook, R. J. \& Sackett, D. L. (1995). The number needed to treat: A clinically useful measure of treatment effect. British Medical Journal 310, 452-454.

Cuijpers, P., Beekman, A. T. F. \& Reynolds Iii, C. F. (2012). Preventing depression: A global priority. JAMA - Journal of the American Medical Association 307, 1033-1034.

Cuijpers, P. \& Smit, F. (2004). Subthreshold depression as a risk indicator for major depressive disorder: A systematic review of prospective studies. Acta Psychiatrica Scandinavica 109, 325-331.

Cuijpers, P., Smit, F., Oostenbrink, J., De Graaf, R., Ten Have, M. \& Beekman, A. (2007). Economic costs of minor depression: A population-based study. Acta Psychiatrica Scandinavica 115, 229-236.

Cuijpers, P., Van Straten, A., Smit, F., Mihalopoulos, C. \& Beekman, A. (2008). Preventing the onset of depressive disorders: A meta-analytic review of psychological interventions. American Journal of Psychiatry 165, 1272-1280.

Cuijpers, P., Van Straten, A., Warmerdam, L. \& Van Rooy, M. J. (2010). Recruiting participants for interventions to prevent the onset of depressive disorders: Possibile ways to increase participation rates. BMC Health Services Research 10, 181.

Dempster, A. P., Laird, N. M. \& Rubin, D. B. (1977). Maximum likelihood from incomplete data via the EM algorithm. Journal of the Royal Statistical Society Series B Methodological 39, 1-38.

Fava, G. A. \& Tomba, E. (2009). Increasing psychological well-being and resilience by psychotherapeutic methods. Journal of Personality $77,1903-1934$.

Fledderus, M., Bohlmeijer, E. T., Smit, F. \& Westerhof, G. J. (2010). Mental health promotion as a new goal in public mental health care: A randomized controlled trial of an intervention enhancing psychological flexibility. American Journal of Public Health 100, 2372-2378. 
Fledderus, M., Oude Voshaar, M. A. H., Ten Klooster, P. M. \& Bohlmeijer, E. T. (2012). Further evaluation of the psychometric properties of the acceptance and action questionnaire-II. Psychological Assessment 24, 925-936.

Haringsma, R., Engels, G. I., Beekman, A. T. F. \& Spinhoven, P. (2004). The criterion validity of the Center for Epidemiological Studies Depression Scale (CES-D) in a sample of self-referred elders with depressive symptomatology. International Journal of Geriatric Psychiatry 19, 558-563.

Hayes, S. C., Luoma, J. B., Bond, F. W., Masuda, A. \& Lillis, J. (2006). Acceptance and Commitment Therapy: Model, processes and outcomes. Behaviour Research and Therapy 44, 1-25.

Horn, P. J. \& Wuyek, L. A. (2010). Anxiety disorders as a risk factor for subsequent depression. International Journal of Psychiatry in Clinical Practice 14, 244-247.

Jacobson, N. S. \& Truax, P. (1991). Clinical significance: A statistical approach to defining meaningful change in psychotherapy research. Journal of Consulting and Clinical Psychology 59, 12-19.

Jané-Llopis, E., Barry, M., Hosman, C. \& Patel, V. (2005). Mental health promotion works: a review. Promotion \& education Suppl 2, 9-25, 61, 67.

Kabat-Zinn, J. (1990). Full catastrophe living: Using the wisdom of your body and mind to face stress, pain and illness. Delacorte: New York.

Kashdan, T. B. \& Rottenberg, J. (2010). Psychological flexibility as a fundamental aspect of health. Clinical Psychology Review 30, 865-878.

Kaviani, H., Hatami, N. \& Javaheri, F. (2012). The impact of Mindfulness-based Cognitive Therapy (MBCT) on mental health and quality of life in a sub-clinically depressed population. Archives of Psychiatry and Psychotherapy 14, 21-28.

Keyes, C. L. M., Dhingra, S. S. \& Simoes, E. J. (2010). Change in level of positive mental health as a predictor of future risk of mental Illness. American Journal of Public Health 100, 2366-2371.

Keyes, C. L. M., Wissing, M., Potgieter, J. P., Temane, M., Kruger, A. \& van Rooy, S. (2008). Evaluation of the mental health continuum-short form (MHC-SF) in Setswana-speaking South Africans. Clinical Psychology and Psychotherapy 15, 181-192.

Klatt, M. D., Buckworth, J. \& Malarkey, W. B. (2009). Effects of low-dose mindfulness-based stress reduction (MBSR-ld) on working adults. Health Education and Behavior 36, 601-614.

Kuyken, W., Byford, S., Taylor, R. S., Watkins, E., Holden, E., White, K., .. Teasdale, J. D. (2008). Mindfulness-Based Cognitive Therapy to Prevent Relapse in Recurrent Depression. Journal of Consulting and Clinical Psychology 76, 966-978.

Kuyken, W., Watkins, E., Holden, E., White, K., Taylor, R. S., Byford, S., ... Dalgleish, T. (2010). How does mindfulness-based cognitive therapy work? Behaviour Research and Therapy 48, 1105-1112.

Lamers, S. M., Westerhof, G. J., Bohlmeijer, E. T., Ten Klooster, P. M. \& Keyes, C. L. (2011). Evaluating the psychometric properties of the mental health Continuum-Short Form (MHC-SF). Journal of Clinical Psychology 67, 99-110.

Lamers, S. M. A., Westerhof, G. J., Kovács, V. \& Bohlmeijer, E. T. (2012). Differential relationships in the association of the Big Five personality traits with positive mental health and psychopathology. Journal of Research in Personality 46, 517-524.

Lipsey, M. W. \& Wilson, D. B. (1993). The efficacy of psychological, educational, and behavioral treatment: Confirmation from meta-analysis. American Psychologist 48, 1181-1209.

Liu, X., Wang, S., Chang, S., Chen, W. \& Si, M. (2013). Effect of Brief Mindfulness Intervention on Tolerance and Distress of Pain Induced by Cold-Pressor Task. Stress and Health 29, 199-204.

Ma, S. H. \& Teasdale, J. D. (2004). Mindfulness-Based Cognitive Therapy for Depression: Replication and Exploration of Differential Relapse Prevention Effects. Journal of Consulting and Clinical Psychology 72, 31-40.

McCarney, R. W., Schulz, J. \& Grey, A. R. (2012). Effectiveness of mindfulness-based therapies in reducing symptoms of depression: A meta-analysis. European Journal of Psychotherapy and Counselling 14, 279-299.

Morris, S. B. \& DeShon, R. P. (2002). Combining effect size estimates in meta-analysis with repeated measures and independent-groups designs. Psychological Methods 7, 105-125. 
Muñoz, R. F., Cuijpers, P., Smit, F., Barrera, A. Z. \& Leykin, Y. (2010). Prevention of major depression. Annual Review of Clinical Psychology 6, 181-212.

Piet, J. \& Hougaard, E. (2011). The effect of mindfulness-based cognitive therapy for prevention of relapse in recurrent major depressive disorder: A systematic review and meta-analysis. Clinical Psychology Review 31, 1032-1040.

Preacher, K. J. \& Hayes, A. F. (2008). Asymptotic and resampling strategies for assessing and comparing indirect effects in multiple mediator models. Behavior Research Methods 40, 879-891.

Radloff, L. S. (1977). The CES-D scale, a self report depression scale for reseach in the general population. Applied Psychological Measurement 1, 385-401.

Ruini, C. \& Fava, G. A. (2012). Role of Well-Being Therapy in Achieving a Balanced and Individualized Path to Optimal Functioning. Clinical Psychology and Psychotherapy 19, 291-304.

Ryff, C. D., Friedman, E. M., Morozink, J. A. \& Tsenkova, V. (2012). Psychological resilience in adulthood and later life: Implications for health. Annual Review of Gerontology and Geriatrics 32, 73-92.

Segal, Z. V., Williams, J. M. G. \& Teasdale, J. D. (2002). Mindfulness-based Cognitive Therapy for Depression: A New Approach to Preventing Relapse. The Guilford Press: New York.

Sheehan, D. V., Lecrubier, Y., Sheehan, K. H., Amorim, P., Janavs, J., Weiller, E., ... Dubar, G. C. (1998). The Mini-International Neuropsychiatric Interview (M.I.N.I.): the development and validation of a structured diagnostic psychiatric interview for DSM-IV and ICD-10. Journal of Clinical Psychiatry 59, 22-33.

Smit, F., Ederveen, A., Cuijpers, P., Deeg, D. \& Beekman, A. (2006). Opportunities for cost-effective prevention of late-life depression: An epidemiological approach. Archives of General Psychiatry $63,290-296$.

Spinhoven, P., Ormel, J., Sloekers, P. P. A., Kempen, G. I. J. M., Speckens, A. E. M. \& Van Hemert, A. M. (1997). A validation study of the hospital anxiety and depression scale (HADS) in different groups of Dutch subjects. Psychological Medicine 27, 363-370.

Strauss, C., Cavanagh, K., Oliver, A. \& Pettman, D. (2014). Mindfulness-based interventions for people diagnosed with a current episode of an anxiety or depressive disorder: A meta-analysis of randomised controlled trials. PLOS ONE 9, eg6rio.

Teasdale, J. D., Segal, Z. V., Williams, J. M. G., Ridgewaya, V. A., Soulsby, J. M. \& Lau, M. A. (2000). Prevention of relapse/recurrence in major depression by mindfulness-based cognitive therapy. Journal of Consulting and Clinical Psychology 68, 615-623.

Van Vliet, I. M. \& De Beurs, E. (2007). The MINI-International Neuropsychiatric Interview (M.I.N.I.). A brief structured diagnostic psychiatric interview for DSM-IV and ICD-10 psychiatric disorders [in Dutch]. Tijdschrift voor Psychiatrie 49, 393-397.

Vøllestad, J., Nielsen, M. B. \& Nielsen, G. H. (2012). Mindfulness- and acceptance-based interventions for anxiety disorders: A systematic review and meta-analysis. British Journal of Clinical Psychology 51, 239-260.

Williams, J. M. G., Crane, C., Barnhofer, T., Brennan, K., Duggan, D. S., Fennell, M. J. V., . . Russell, I. T. (2014). Mindfulness-based cognitive therapy for preventing relapse in recurrent depression: A randomized dismantling trial. Journal of Consulting and Clinical Psychology 82, 275-286.

Wood, A. M. \& Joseph, S. (2010). The absence of positive psychological (eudemonic) well-being as a risk factor for depression: A ten year cohort study. Journal of Affective Disorders 122, 213-217.

Zigmond, A. S. \& Snaith, R. P. (1983). The hospital anxiety and depression scale. Acta Psychiatrica Scandinavica 67, 361-370. 


\section{CHAPTER 3}
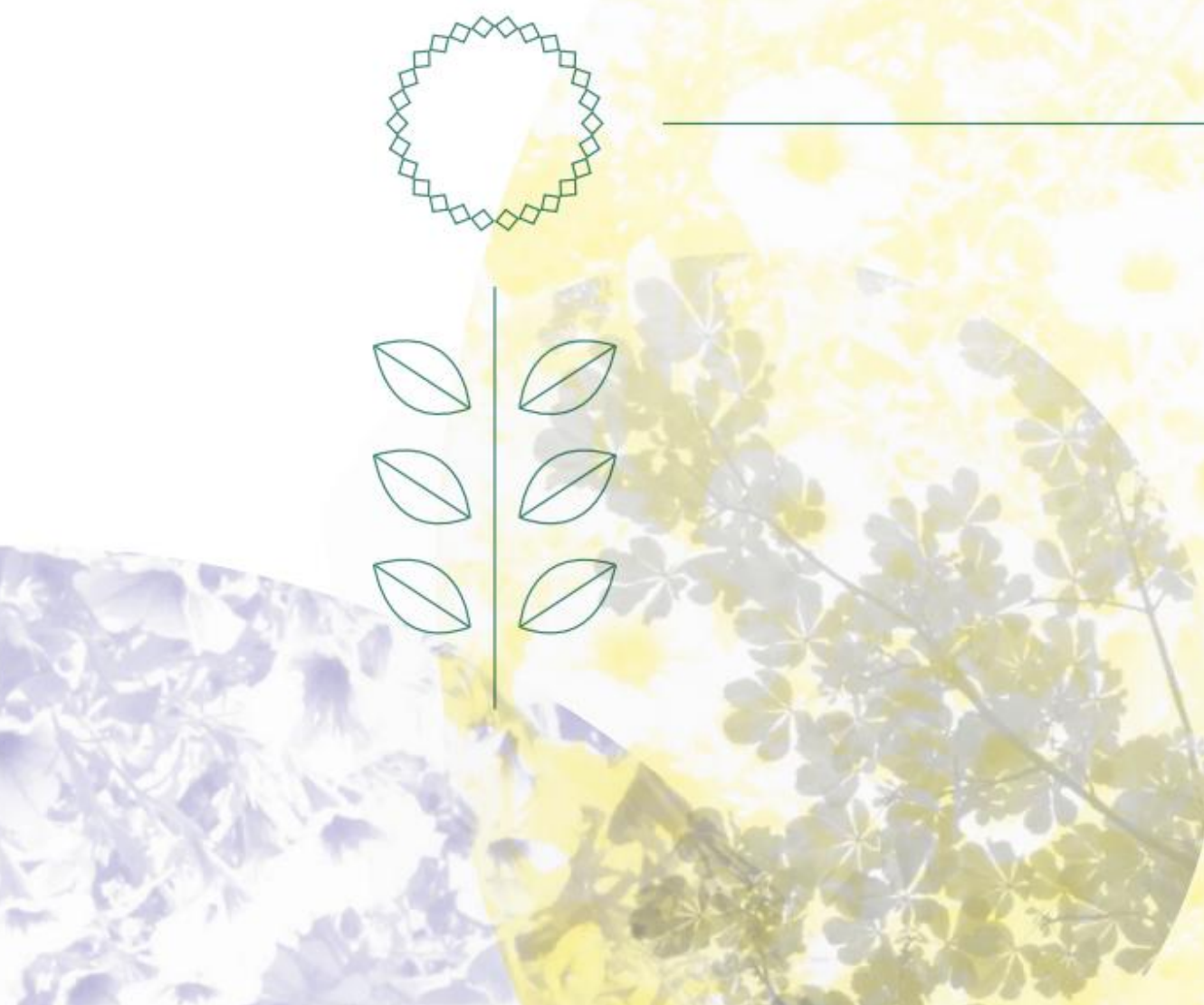

ye:-

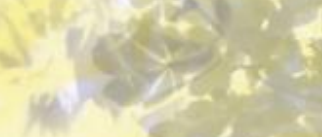

1002.

ate $x^{3}$ 


\section{Effectiveness of online mindfulness-}

based interventions in improving mental health: a review and meta-analysis of randomised controlled trials

Spijkerman, M.P.J., Pots, W.T.M. \& Bohlmeijer, E.T. (2016). Effectiveness of online mindfulness-based interventions in improving mental health: a review and metaanalysis of randomised controlled trials.

Clinical Psychology Review 45, 102-114
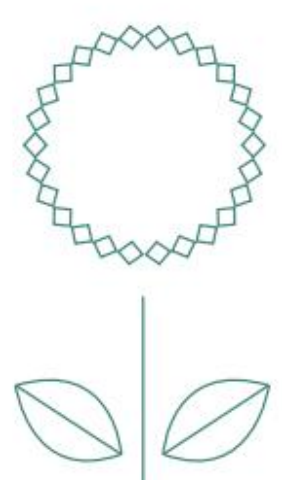


\section{Abstract}

Mindfulness-based interventions (MBIs) are increasingly being delivered through the Internet. Whereas numerous meta-analyses have investigated the effectiveness of face-to-face MBIs in the context of mental health and well-being, thus far a quantitative synthesis of the effectiveness of online MBIs is lacking. The aim of this meta-analysis was to estimate the overall effects of online MBIs on mental health. Fifteen randomised controlled trials were included in this study. A random effects model was used to compute pre-post between-group effect sizes, and the study quality of each of the included trials was rated. Results showed that online MBIs have a small but significant beneficial impact on depression $(g=0.29)$, anxiety ( $g=0.22$ ), well-being $(g=0.23$ ) and mindfulness ( $g=0.32)$. The largest effect was found for stress, with a moderate effect size $(g=0.51)$. For stress and mindfulness, exploratory subgroup analyses demonstrated significantly higher effect sizes for guided online MBIs than for unguided online MBIs. In addition, meta-regression analysis showed that effect sizes for stress were significantly moderated by the number of intervention sessions. Effect sizes, however, were not significantly related to study quality. The findings indicate that online MBIs have potential to contribute to improving mental health outcomes, particularly stress. Limitations, directions for future research and practical implications are discussed. 


\section{Introduction}

Although mindfulness has been employed for centuries within Buddhist traditions, it is only since the 1970's that mindfulness has become a target of therapeutic intervention for common psychological problems such as stress, worry, anxiety and depression (Keng, Smoski, \& Robins, 2011). Mindfulness could be defined as the ability to observe thoughts, bodily sensations or feelings in the present moment with an open and accepting orientation toward one's experiences (Bishop et al., 2004; Kabat-Zinn, 1990). Currently, mindfulness practices have been incorporated into various therapies in the field of mental health care, such as Mindfulness-Based Stress Reduction (MBSR; Kabat-Zinn, 1982; Kabat-Zinn, 1990), Mindfulness-Based Cognitive Therapy (MBCT; Segal, Williams, \& Teasdale, 2002), Dialectical Behaviour Therapy (DBT; Linehan, 1993), and Acceptance and Commitment Therapy (ACT; Hayes, Strosahl, \&Wilson, 1999). Through facilitating awareness and non-judgmental acceptance of moment-to-moment experiences, these mindfulness-based interventions (MBIs) are assumed to alleviate intense emotional states (Baer, 2003; Keng et al., 2011). Extensive descriptions of MBSR, MBCT, DBT and ACT as well as their underlying mechanisms of change can be found elsewhere (Baer, 2003; Bishop, 2002; Feigenbaum, 2007; Hayes, Luoma, Bond, Masuda, \& Lillis, 2006; Metcalf \& Dimidjian, 2014; Praissman, 2008; Ruiz, 2010).

In the past two decades, MBIs have become increasingly popular (Baer, 2003; Keng et al., 2011). Along with this growing interest in MBIs, there has been an exponential increase in the number of studies addressing the non-clinical and clinical utility of these interventions. As evidenced by a substantial number of meta-analyses, MBIs have proven effective in reducing psychological distress, most notably anxiety and depression, and improving well-being and quality of life in a broad range of populations, including healthy populations (Chiesa \& Serretti, 2009; Khoury, Sharma, Rush, \& Fournier, 2015), individuals with mental disorders (Chiesa \& Serretti, 2011; Klainin-Yobas, Cho, \& Creedy, 2012; McCarney, Schulz, \& Grey, 2012; Piet \& Hougaard, 2011; Strauss, Cavanagh, Oliver, \& Pettman, 2014; Vøllestad, Nielsen, \& Nielsen, 2012) and individuals suffering from chronic somatic illnesses (Abbott et al., 2014; Bohlmeijer, Prenger, Taal, \& Cuijpers, 2010; Cramer, Lauche, Paul, \& Dobos, 2012; Lauche, Cramer, Dobos, Langhorst, \& Schmidt, 2013; Ledesma \& Kumano, 2009; Piet, Würtzen, \&Zachariae, 2012; Veehof, Oskam, Schreurs, \& Bohlmeijer, 2011; Zainal, Booth, \& Huppert, 2013).

Previous meta-analyses have reported inconsistent findings with regard to the effects of MBIs on depression and anxiety, with effect sizes varying between 0.3 and o.8 (Abbott et al., 2014; Bohlmeijer et al., 2010; Cavanagh, Strauss, Forder, \& Jones, 2014; Cramer et al., 2012; Hofmann, Sawyer, Witt, \& Oh, 2010; Khoury et al., 2015; 
Klainin-Yobas et al., 2012; McCarney et al., 2012; Piet, Würtzen, et al., 2012; Strauss et al., 2014; Veehof et al., 2011; Vøllestad et al., 2012; Zainal et al., 2013). There are also multiple meta-analyses that have assessed the impact of MBIs on stress with effect sizes ranging from 0.4 to o.7 (Abbott et al., 2014; De Vibe, Bjørndal, Tipton, Hammerstrøm, \& Kowalski, 2012; Khoury et al., 2015; Zainal et al., 2013). Effects on mindfulness, as found in several earlier meta-analyses are more consistent and in the moderate range, between approximately 0.4 and 0.5 (Cavanagh et al., 2014; Khoury et al., 2015; Piet, Würtzen, et al., 2012; Visted, Vøllestad, Nielsen, \& Nielsen, 2014). More recently, Gotink et al. (2015) synthesized the results of meta-analyses that investigated the effectiveness of MBSR and MBCT as compared to waitlist controls and treatment as usual in different populations. They found an effect size of $0.37,0.49,0.51$ and 0.39 for depression, anxiety, stress and quality of life, respectively.

Not surprisingly, given the rapid development of information technologies, MBIs - like other psychotherapeutic interventions - are increasingly being delivered through the Internet. Online interventions have a number of advantages over face-to-face interventions. Online interventions: 1) are easily accessible, without long waiting lists; 2) available 24/7 to people in their own environment, saving traveling time and enabling people to work at their own pace; 3) permit users to remain anonymous without needing to adopt a patient role; 4) do not necessarily require involvement of a therapist educated in mindfulness; and 5) are less costly (Andersson \& Titov, 2014; Cuijpers et al., 2009). Moreover, a cross-sectional survey among 500 adults in the United States showed that many people prefer individual and online formats for mindfulness meditation interventions above group formats (Wahbeh, Svalina, \& Oken, 2014). The internet was found to be the first choice format for $42 \%$ of the participants, suggesting that, for many individuals, online MBIs may be an acceptable alternative to face-to-face formats.

While multiple randomised controlled trials (RCTs) have provided empirical evidence for the effectiveness of online MBIs in the context of mental health and well-being (e.g. Boettcher et al., 2014; Buhrman et al., 2013; Dowd et al., 2015; Ly et al., 2014; Pots et al., 2015; Trompetter, Bohlmeijer, Veehof, \& Schreurs, 2014; Zernicke et al., 2014), to our knowledge, no published meta-analyses have examined the specific effects of online-delivered MBIs on mental health outcomes. However, two published meta-analyses investigating the effects of MBIs did include studies that employed online interventions. The first investigated the impact of self-help interventions, including components of mindfulness, on mindfulness/acceptance, depression and anxiety (Cavanagh et al., 2014). Cavanagh et al. (2014) found that self-help interventions that included components of mindfulness had a beneficial impact on mindfulness/acceptance skills $(g=0.49)$, anxiety $(g=-0.33)$ and depression $(g=-0.37)$ compared to control conditions. Although the meta-analysis conducted 
by Cavanagh and colleagues (2014) included eight (out of fifteen) studies that used an online intervention (of which four were multi-component interventions), their findings were inconclusive regarding the effectiveness of online-delivered MBIs. The second meta-analysis conducted by Öst (2014) evaluated the effectiveness of ACT across various psychiatric and somatic disorders. This study, however, only used the primary outcome measure, resulting in an overall effect size of $g=0.42$ (Öst, 2014). In addition, only three of the sixty RCTs included in the study exclusively used online intervention. Finally, the meta-analysis of Öst (2014) did not examine the separate effects of ACT on depression, anxiety, stress or well-being nor the specific effects of online MBIs.

Since the publication of these two meta-analyses, both of which included data collected up until November 2013, a number of RCTs investigating the effectiveness of online MBIs have appeared in the scientific literature (e.g. Dowd et al., 2015; Pots et al., 2015; Trompetter et al., 2014; Zernicke et al., 2014). Based on the fact that most studies investigating the effects of online MBIs have been published in the last three years, and that interventions delivered through the Internet, in general, receive considerable attention nowadays (Barak, Klein, \& Proudfoot, 2009), we anticipate a further rise in the number of online-delivered MBIs in the upcoming years. Hence, we consider it timely and important to meta-analytically test the effectiveness of online MBIs in terms of mental health outcomes. Accordingly, the primary aim of this explorative meta-analysis was to estimate the overall effect of online MBIs on depression, anxiety, stress and well-being, in accordance with the Preferred Reporting Items for Systematic Reviews and Meta-Analyses (PRISMA) statement (Moher et al., 2009). MBSR, MBCT and ACT are the most frequently studied online MBIs and also the focus of this article. Since MBIs are based on the premise that enhancing mindfulness skills will contribute to better mental health outcomes, our secondary aim was to explore the effects of online MBIs on mindfulness.

\section{Method}

This study was conducted in accordance with the PRISMA statement for reporting systematic reviews and meta-analyses of studies that evaluate health care interventions (Moher et al., 2009).

\section{Search strategy}

A systematic literature search was conducted in three electronic databases: PsycINFO, PubMed and Web of Science. Each database was initially searched for English language journal articles from the first available date until 27 November 2014, using the following search terms: (mindful* or acceptance or meditation) and (intervention* 
or therap* or treatment* or program*) and (online or e-health or Internet* or web* or computer or app or apps) and (random* or trial or RCT or control*). In PsycINFO and PubMed, MesH terms and thesaurus terms were added, respectively (see the Appendix for the full search strings). During the preparation of the meta-analysis, the search was repeated three times to identify newly published trials. The last search was conducted on 23 March 2015.

In addition, three clinical trial registers (www.clinicaltrialsregister.eu, www.isrctn.com, and www.clinicaltrials.gov) were searched on 26 February 2015, to detect completed trials that had not yet been published (see the Appendix for the full search strings). We contacted six authors of potentially relevant records of which one author responded.

\section{Selection of studies}

After the removal of duplicates, the remaining titles were reviewed, and then the abstracts of the potentially relevant articles were screened. Finally, the full-texts of the selected articles were obtained and assessed for eligibility. The screening of titles, abstracts and full-text articles, respectively, was independently conducted by two authors (MS, WP). Disagreements between the authors were discussed until consensus was reached. If any disagreement persisted, the last author (EB) was consulted.

Due to the explorative nature of this meta-analysis, we opted for rather broad inclusion criteria. We included studies that: 1 ) employed MBIs (including MBSR, $\mathrm{MBCT}$ and $\mathrm{ACT}$ ) either with or without guidance; 2) administered the MBI via the Internet or a computer application (including virtual classrooms); 3) used validated outcome measures to examine the effects of the intervention on depression, anxiety, stress or well-being; 4) administered the intervention to a population 18 years and older; 4) used a control condition whether inactive or active; and 5) used a randomised controlled design.

Exclusion criteria were: 1) The intervention was merely a psycho-educational program and did not involve exercises for enhancing mindfulness or acceptance; 2) The intervention combined MBI and other forms of therapy (e.g. cognitive behavioural therapy), making it difficult to disentangle the effects of the MBI from the other included therapies; 3) The article did not provide sufficient data to calculate pre-post effect sizes per condition and the author was unable to provide this necessary data.

Five authors were contacted, all of whom provided additional data on request.

\section{Data extraction and quality assessment}

Data extraction was undertaken by the first author (MS) and checked by the second author (WP). Disagreements were resolved by discussion. For each included study, the following data were extracted: first author; country and year of publication; 
population characteristics, including type of sample, age, sex (\% female) and number of participants per condition; intervention characteristics, including type of intervention (e.g. MBSR, MBCT, ACT), guidance (with/without), delivery mode (e.g. website), number of sessions and duration in weeks; control group (e.g. waitlist); assessment times (i.e. pre, post, follow-up); and outcome measures for depression, anxiety, stress, well-being and mindfulness.

The methodological quality of each study was independently assessed by two authors (MS, WP), who used seven criteria based on the Jadad scale (Jadad et al., 1996) and the Cochrane Collaboration's tool for assessing risk of bias (Higgins, Altman, \&Sterne, 2011). The following criteria were applied: 1) adequate sequence generation and allocation concealment; 2) blinding of main outcome assessments, that is, outcome measures were administered online or by an independent person who was not involved in the study (blinding of participants was not possible in most cases); 3) reasons for drop-out and withdrawal were described; 4) handling of missing data, that is, intention-to-treat analyses were conducted, in which all randomised participants were included, or there were no drop-outs; 5) the sample size was based on an adequate power analysis; 6) study groups were similar with regard to prognostic indicators at baseline and this was explicitly assessed, or adjustments were made to correct for baseline imbalance; 7) diagnostic assessment of the primary outcome was conducted by a professional (not by self-reporting or screening), or there were no diagnostic assessments necessary for the recruitment (e.g. students).

One point was assigned for each criterion that was met, with a maximum score of 7. Disagreements between the two authors who assessed the quality of the studies were resolved by discussion. The quality of a study was assessed as "high" when all seven criteria were met, "medium" when five or six criteria were met, and "low" when four or less criteria were met.

Twelve authors were contacted because insufficient information was provided in the article with regard to the data extraction and/or to make an accurate quality assessment. Consequently, ten authors provided supplementary information.

\section{Calculation of effect sizes}

For each comparison between an online MBI and a control group, effect sizes were calculated per outcome variable, i.e. depression, anxiety, stress and well-being. For well-being, we also used instruments related to well-being such as life satisfaction (e.g. SWLS, QOLI). If more than one instrument was used to measure depression, anxiety, stress or well-being, we used the most valid instrument, so that each study outcome had one effect size. One study (Cavanagh et al., 2013) used the PHQ-4 to measure depression and anxiety. Since this questionnaire does not allow to calculate separate scores for depression and anxiety, we excluded this questionnaire. Addi- 
tionally, we calculated effect sizes for mindfulness measures whenever possible.

Two studies investigated the effectiveness of two different online MBIs compared to the same control group (Mak, Chan, Cheung, Lin, \& Ngai, 2015; Morledge et al., 2013). In these cases, we calculated an effect size for both comparisons. On the other hand, for the three studies that included two control groups and one experimental group (Pots et al., 2015; Trompetter et al., 2014; Wolever et al., 2012), we used only one control group to calculate an effect size. For these studies, we chose the inactive control condition (i.e. waitlist or no intervention) as this was the most common comparison group across all the studies. The number of studies using an active control condition (Pots et al., 2015; Trompetter et al., 2014) was too small to allow for subgroup analyses based on the type of control group (i.e. inactive versus active).

For each comparison, Hedge's g, i.e. Cohen's d corrected for small sample bias, was calculated per relevant outcome measure, using means and standard deviations. First, we calculated standardised pre-post effect sizes, using the formula $d=\left(M_{1}-M_{0}\right)$ $/ \mathrm{SD}_{\mathrm{o}}$, where $\mathrm{M}_{1}$ and $\mathrm{M}_{\mathrm{o}}$ are the Means at post- and pre-test, respectively, and $\mathrm{SD}_{\mathrm{o}}$ is the pre-test standard deviation. Since we were interested in obtaining the effect size of the experimental effect minus the effect in the control group, we calculated $d$ per condition, i.e. for the experimental condition $\left(d_{E}\right)$ and the control condition $\left(d_{c}\right)$. These ds represent how many standard deviations difference there is between the means of the pre- and the post-test of the respective condition. Subsequently, we calculated the difference between $d_{E}$ and $d_{C}, \Delta(d)$, which shows us with how many standard deviations the experimental condition changed more compared to the control condition. Finally, using the software program Comprehensive Meta Analysis (CMA) version 2.2.064, $\Delta$ (d) was corrected for small sample bias, indicated as Hedge's g. Values of g can be interpreted in a similar manner as values of d. Using a second-order meta-analysis, Lipsey and Wilson (1993) have shown that an effect size from 0.00 to 0.32 can be considered a small effect, 0.33 to 0.55 a moderate effect and 0.56 to 1.20 a large effect. Because there was too much variability in follow-up periods, we did not calculate effect sizes of the change between pre-test and (longerterm) follow-up.

\section{Meta-analytic procedures}

All meta-analytic analyses were conducted with CMA version 2.2.064. Due to the diversity in intervention and population characteristics (see Table 1) and the rather broad inclusion criteria, we expected considerable variability in effect sizes and levels of heterogeneity. Consequently, it was decided a priori to use the random effects model. The random effects model is based on the assumption that the effect size may differ between studies not only due to random error within studies, but also as a result of true variation in effect sizes between studies (Hedges \& Vevea, 1998). 
Five separate meta-analyses were performed for 1) depression, 2) anxiety, 3) stress, 4) well-being, and 5) mindfulness. Forest plots of pre-post between-group effect sizes were produced for each outcome variable, both with and without outliers. A study was considered an outlier when its $95 \%$ confidence interval (CI) was outside the $95 \% \mathrm{CI}$ of the overall mean effect size (on both sides). Outliers were identified through visual inspection of the forest plots. Subsequently, the analyses were repeated, but only with medium and high quality studies (including outliers).

Heterogeneity of effect sizes was examined using $Q$ and $I^{2}$ statistics. A significant $Q$ statistic $(p \leq 0.05)$ indicated significant heterogeneity, i.e. the presence of one or more variables that moderated the observed effect size. The $\mathrm{I}^{2}$ statistic was used to estimate the percentage of heterogeneity across the primary studies not attributable to random sample error alone. A value of $\% \%$ indicated no heterogeneity. Values of $25 \%, 50 \%$ and $75 \%$ reflected low, moderate and high degrees of heterogeneity, respectively (Higgins \& Thompson, 2002).

Pre-specified exploratory subgroup analyses were performed (including outliers) to examine differences in effect sizes based on: 1) intervention type: mindfulness or $\mathrm{ACT}$; 2) therapist guidance: with or without; and 3) population: healthy, psychological symptoms, or physical symptoms. The moderating effects of the study quality and number of intervention sessions on effect sizes were assessed using meta-regression analyses, according to the mixed effects model.

Publication bias was assessed in three ways. First, a funnel plot was created by plotting the overall mean effect size against study size. Whereas a symmetric distribution of studies around the effect size indicates the absence of publication bias, a higher concentration of studies on one side of the effect size than on the other indicates publication bias (Sterne, Egger, \& Moher, 2008). Second, a fail-safe N, a formal test of funnel plot asymmetry, was calculated for each analysis. The fail-safe $\mathrm{N}$ indicates the number of unpublished non-significant studies that would be required to lower the overall effect size below significance (Egger, Davey Smith, Schneider, \& Minder, 1997). The findings were considered robust if the fail-safe $N \geq 5 n+10$, where $\mathrm{n}$ is the number of comparisons (Rosenberg, 2005). Third, Duval and Tweedie's (2000) trim-and-fill procedure was applied. This procedure imputes the effect sizes of missing studies and produces an adjusted effect size accounting for these missing studies (Duval \& Tweedie, 2000). 


\section{Results}

\section{Selection of studies}

A flow diagram of the study selection process is presented in Figure 1. The electronic database searches produced 805 records after removal of duplicates. After reviewing the titles, we identified 150 potentially eligible records. Based on the abstracts, 34 of these 150 articles were selected for further examination. Full-text versions of these articles were obtained and assessed for eligibility. This led to the inclusion of 15 RCTs, totalling 17 comparisons of an online MBI with a control group (in two trials, two comparisons are made using a single control group). Additionally, 176 records were identified through searching trial registers, of which seven were assessed as potentially relevant. No unpublished data were made available.

\section{Description of included studies}

Four studies were conducted in the United States, four in Sweden, two in The Netherlands, and one each in the United Kingdom, Ireland, Austria/Switzerland, China, and Canada. Characteristics of the included trials are presented in Table 1.

\section{Population characteristics}

The total population comprised 236o participants of which 1211 participants were in the experimental conditions and 1149 in the control conditions ( 913 when excluding the control conditions not included in the meta-analysis). In all but one study (Hesser et al., 2012), the majority of the sample was female. All participants were adults, with a mean age ranging from 18 to 58 years. The total sample size ranged from 49 in a pilot study (Glück \& Maercker, 2011) to 551 in a large-scale trial (Morledge et al., 2013). Five of the 15 studies were conducted in a population with a somatic illness, including chronic pain $(n=3)$, tinnitus $(n=1)$ and cancer recovery patients $(n=1)$. In three studies, participants were characterized by psychological illnesses, i.e. anxiety $(n=1)$ or depression $(n=2)$. Non-clinical populations, such as students or employees, were used in the remaining seven studies.

\section{Intervention characteristics}

Eight of the 17 comparisons examined MBSR, two MBCT and five ACT. The 10 comparisons examining MBSR or MBCT used modified protocols instead of pure MBSR or $\mathrm{MBCT}$, in the sense that the intervention: 1) comprised more or less than eight sessions; 2) used shortened exercises; 3) was adapted to a specific target population (e.g. cancer recovery patients); and/or 4) did not involve a retreat. Two comparisons used an Internet-based mindfulness treatment, which could not be classified as MBSR or MBCT (Boettcher et al., 2014; Cavanagh et al., 2013). In nine comparisons, 


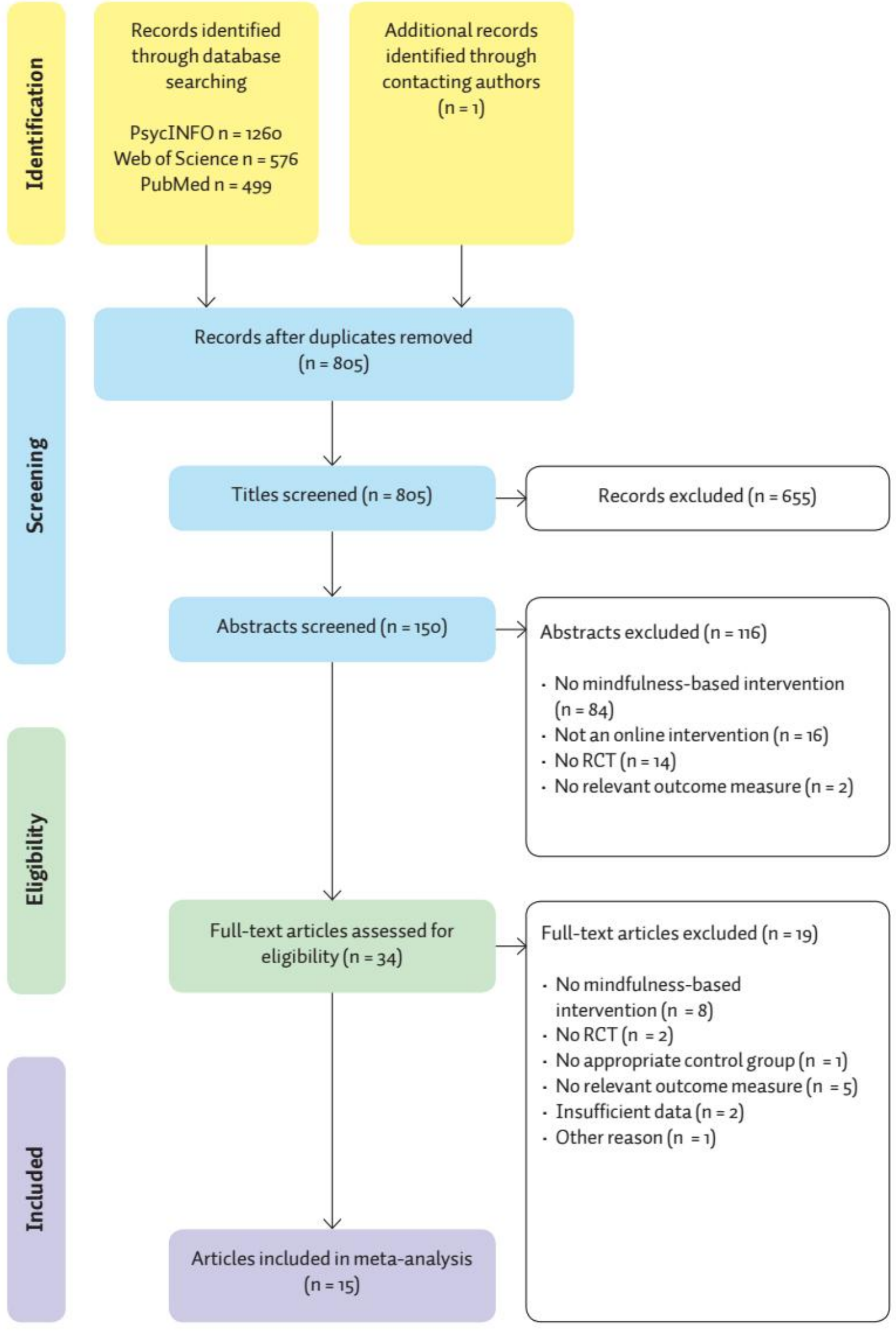

Figure 1 - Flowchart of the study selection process. 
therapist guidance was offered during the intervention. In five of these comparisons, guidance consisted of individual coaching and feedback (e.g. answering questions, feedback on assignments, positive encouragement) delivered through e-mail, an enclosed and encrypted webpage and/or telephone. In three comparisons, guidance was provided in the form of weekly 1- or 2-hour (online) classes (group-based), of which one study additionally provided (pre-programmed) individual e-mail coaching and feedback. In one comparison, participants were reinforced through messages posted on an online message board. MBIs were most commonly delivered via a website $(n=14)$. Other delivery modes included a smartphone application $(n=1)$ and a virtual online classroom ( $n=2)$. One comparison (Aikens et al., 2014) used a combination of a website and a virtual online classroom. Sessions were usually weekly, ranging from 2 to 12 sessions. The intervention duration varied from 2 to 12 weeks.

\section{Adherence}

Adherence to the intervention was addressed in ten studies, using various definitions of adherence (e.g. $100 \%$ of the sessions completed, $\geq 5$ sessions completed, or $6-8$ weeks). When adherence was defined as completion of all sessions, adherence rates varied between $39.5 \%$ and $92 \%$ (based on five studies).

\section{Comparison group}

Nine studies compared an online MBI to a waitlist control group, of which two studies (Pots et al., 2015; Trompetter et al., 2014) also included an active control group (i.e. expressive writing). In five studies, the control group received access to an online discussion forum $(n=3)$, a psycho-educational program $(n=1)$, or a behavioural activation program $(n=1)$. In the remaining study, the control group received no intervention.

\section{Outcomes}

Outcome measures were administered as follows: depression in 12 comparisons, anxiety in 11 comparisons, stress in 11 comparisons, well-being in 9 comparisons and mindfulness in 12 comparisons. All instruments had good psychometric properties. Eight studies reported follow-up data, with follow-up periods varying between 12 weeks and one year.

\section{Quality of studies}

The quality assessment scores ranged from 3 to 7 points (see Table 2). Most studies $(n=10)$ were of medium quality, three of low quality and two of high quality. All studies met the criteria of blinding and intention-to-treat analysis. Description of withdrawals/drop-outs (Criterion 3) was the most poorly rated, with only three studies meeting this criterion. 


\section{Meta-analysis}

The pre-post between-group effects for depression, anxiety, stress, well-being and mindfulness are presented in Table 3 . Below, the results are discussed per outcome measure.

\section{Effects on depression}

For depression (12 comparisons), a significant, small effect was observed ( $g=0.29$, $95 \% \mathrm{CI}: 0.13$ to $0.46, \mathrm{p}=0.001)$. The level of heterogeneity was moderate $\left(\mathrm{I}^{2}=58.35\right)$. Two outliers were detected (Boettcher et al., 2014; Ly et al., 2014). After omitting these studies from the analysis, we found a similar effect, with $g=0.27$ (95\% CI: 0.16 to $0.39, \mathrm{p}<0.001)$, and heterogeneity reduced substantially $\left(\mathrm{I}^{2}=6.33\right)$. When only studies scored as medium or high quality were included in the analysis (including outliers), a similar significant effect size was observed ( $g=0.28,95 \%$ CI: 0.08 to 0.47 , $p=0.005)$, with a substantial level of heterogeneity $\left(I^{2}=65.29\right)$.

\section{Effects on anxiety}

Based on 11 comparisons, we found a significant, small effect of online MBIs on anxiety, with $g=0.22$ ( $95 \% \mathrm{CI}: 0.05$ to $0.39, \mathrm{p}=0.010$ ) and no outliers. The level of heterogeneity was moderate $\left(I^{2}=56 \cdot 98\right)$. After removal of low quality studies from the analysis, the effect size was virtually the same ( $g=0.21,95 \% \mathrm{CI}: 0.03$ to 0.40 , $\mathrm{p}=0.022)$, and heterogeneity remained substantial $\left(\mathrm{I}^{2}=60.58\right)$.

\section{Effects on stress}

For stress (11 comparisons), a significant, moderate effect was found ( $g=0.51,95 \%$ $\mathrm{CI}: 0.26$ to $0.75, \mathrm{p}<0.001)$. Heterogeneity was considerable $\left(\mathrm{I}^{2}=82.46\right)$, and one outlier was detected (Wolever et al., 2012). After removal of the outlier, the effect size dropped to $g=0.39$ ( $95 \% \mathrm{CI}: 0.21$ to $0.57, \mathrm{p}<0.001$ ), but still remained in the moderate range, and the level of heterogeneity remained high $\left(\mathrm{I}^{2}=65.63\right)$. Also when studies of low quality were omitted from the analysis, the effect size for stress was in the moderate range ( $g=0.40,95 \% \mathrm{CI}: 0.20$ to $0.59, \mathrm{p}<0.001$ ), with substantial heterogeneity $\left(I^{2}=69 \cdot 41\right)$.

\section{Effects on well-being}

The overall mean effect size for 9 comparisons on well-being was $g=0.23$ ( $95 \% \mathrm{CI}$ : 0.09 to 0.38$)$. This effect was statistically significant $(\mathrm{p}=0.001)$ and can be considered a small effect. The level of heterogeneity was low to moderate $\left(\mathrm{I}^{2}=32.86\right)$, and no outliers were identified. After removal of low quality studies, the effect size for wellbeing slightly increased to $g=0.25$ ( $95 \% \mathrm{CI}$ : 0.10 to $0.40, p=0.001$ ), and heterogeneity was moderate $\left(I^{2}=36 \cdot 16\right)$. 


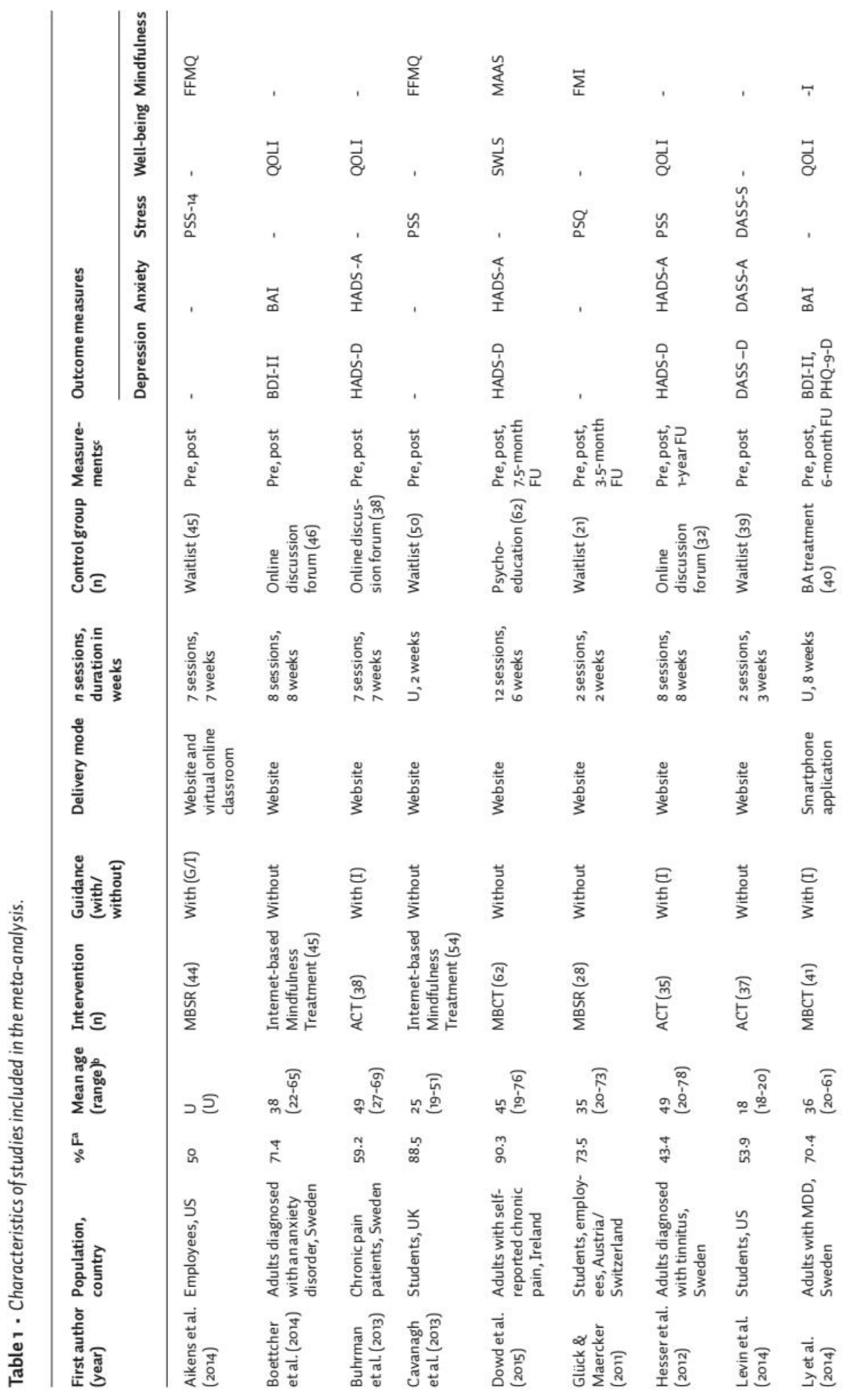




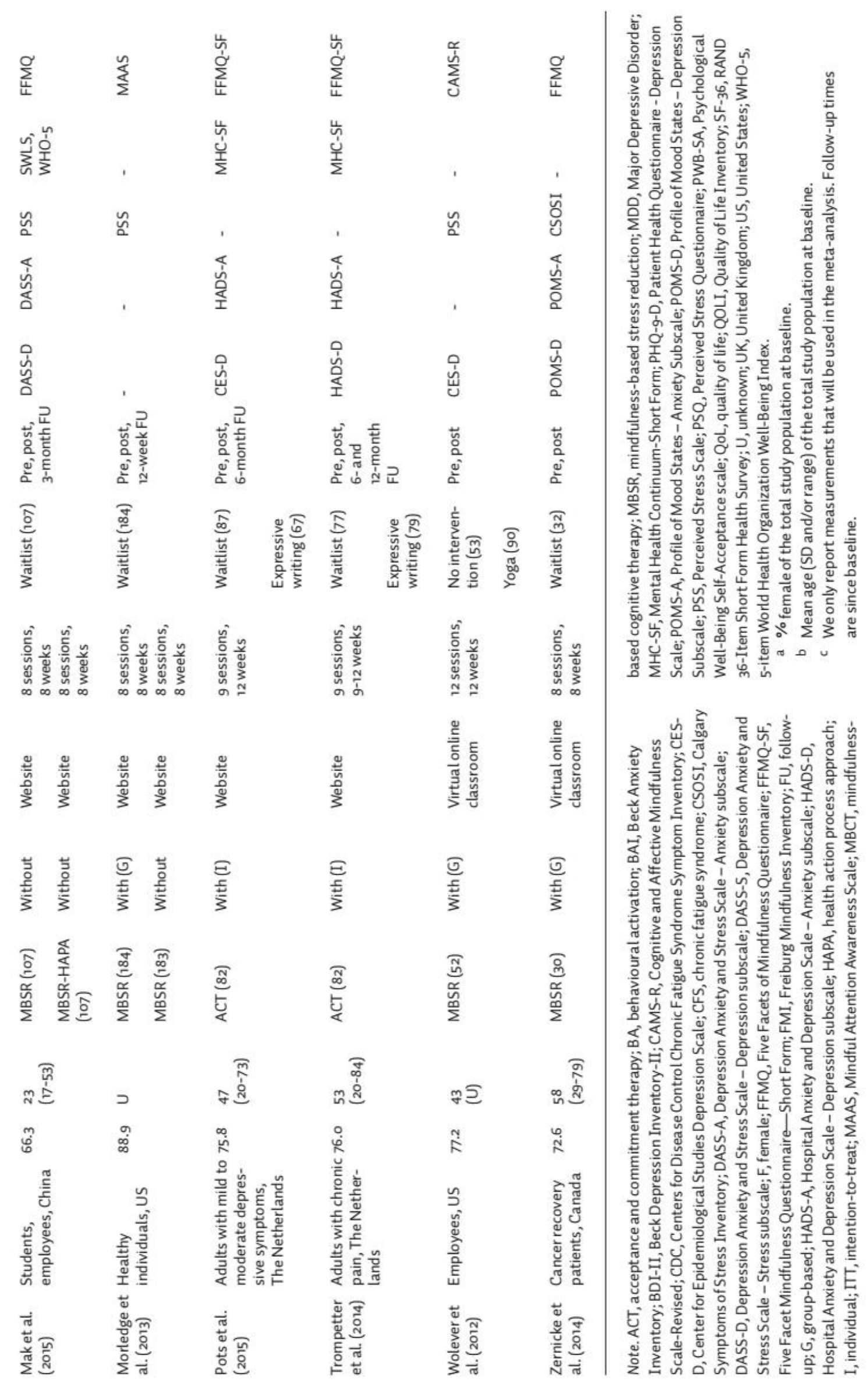




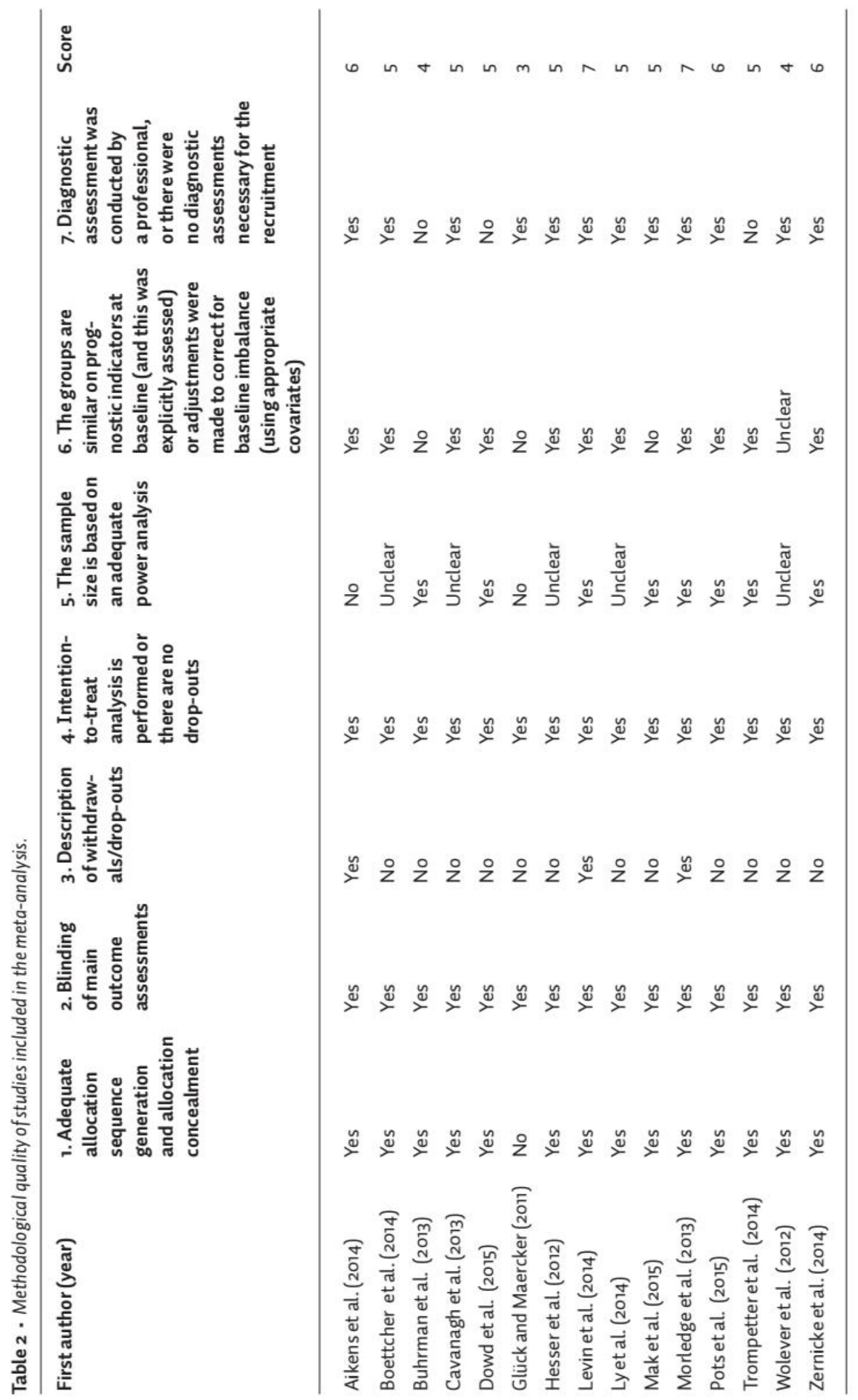


Table 3 - Pre-post between-group effects.

\begin{tabular}{|c|c|c|c|c|c|c|c|}
\hline \multirow[b]{2}{*}{ Outcome measures } & \multirow[t]{2}{*}{$\mathrm{N}_{\text {comp }}$} & \multirow{2}{*}{$\begin{array}{c}\text { Hedge's } \\
\text { g }\end{array}$} & \multirow[t]{2}{*}{$95 \% \mathrm{CI}$} & \multirow[t]{2}{*}{ Z } & \multicolumn{2}{|c|}{ Heterogeneity } & \multirow{2}{*}{$\begin{array}{c}\text { Fail-safe } \\
\text { N }\end{array}$} \\
\hline & & & & & Q-value & $\mathrm{I}^{2}$ & \\
\hline \multicolumn{8}{|c|}{ All studies (including outliers) } \\
\hline Depression & 12 & 0.29 & 0.13 to 0.46 & $3.44^{* *}$ & $26.41^{* *}$ & 58.35 & 76 \\
\hline Anxiety & 11 & 0.22 & 0.05 to 0.39 & $2.58^{\star}$ & $23.25^{\star}$ & 56.98 & 33 \\
\hline Stress & 11 & 0.51 & 0.26 to 0.75 & $4.07^{\star \star \star}$ & $57.01^{* \star *}$ & 82.46 & 225 \\
\hline Well-being & 9 & 0.23 & 0.09 to 0.38 & $3.23^{\star \star}$ & 11.92 & 32.86 & 28 \\
\hline Mindfulness & 12 & 0.32 & 0.23 to 0.42 & $6.60^{\star \star \star}$ & 12.53 & 12.23 & 145 \\
\hline \multicolumn{8}{|c|}{ All studies (excluding outliers) } \\
\hline Depression & 10 & 0.27 & 0.16 to 0.39 & $4.67^{\star \star \star}$ & 9.61 & 6.33 & 56 \\
\hline Stress & 10 & 0.39 & 0.21 to 0.57 & $4.20^{\star \star \star *}$ & $26.19^{\star *}$ & 65.63 & 125 \\
\hline Mindfulness & 11 & 0.30 & 0.21 to 0.39 & $6.51^{\star \star \star}$ & 6.62 & o & 101 \\
\hline \multicolumn{8}{|c|}{ Medium and high quality studies } \\
\hline Depression & 10 & 0.28 & 0.08 to 0.47 & $2.80^{\star *}$ & $25.93^{\star \star}$ & 65.29 & 48 \\
\hline Anxiety & 10 & 0.21 & 0.03 to 0.40 & $2.30^{*}$ & $22.83^{\star}$ & 60.58 & 24 \\
\hline Stress & 9 & 0.40 & 0.20 to 0.59 & $4.00^{* \star *}$ & $26.15^{\star \star}$ & 69.41 & 114 \\
\hline Well-being & 8 & 0.25 & 0.10 to 0.40 & $3.29^{\star \star}$ & $10.96^{\star *}$ & 36.16 & 29 \\
\hline Mindfulness & 10 & 0.32 & 0.21 to 0.43 & $5.78^{\star \star *}$ & 12.26 & 26.60 & 114 \\
\hline
\end{tabular}

Note. $\mathrm{N}_{\text {comp }}$, number of comparisons; $\mathrm{CI}$, confidence interval.

${ }^{\star} \mathrm{p}<0.05,{ }^{\star \star} \mathrm{p}<0.01,{ }^{\star \star *} \mathrm{p}<0.001$.

\section{Effects on mindfulness}

For mindfulness, we were able to compare the effects of an online MBI to a control condition in 10 studies, totalling 12 comparisons. The findings revealed that online MBIs have a significant impact on mindfulness, with a small effect size of $g=0.32$ ( $95 \%$ CI: 0.23 to $0.42, \mathrm{p}<0.001$ ). Heterogeneity was low $\left(\mathrm{I}^{2}=12.23\right.$ ). One outlier was identified (Aikens et al., 2014). After removal of this outlier, the observed effect size was virtually the same ( $g=0.30,95 \% C I$ : 0.21 to $0.39, p<0.001$ ), with absence of heterogeneity $\left(\mathrm{I}^{2}=0\right)$. When we included only studies of medium or high quality in the analysis, we found the same effect size for mindfulness ( $g=0.32,95 \% \mathrm{CI}: 0.21$ to $0.43, \mathrm{p}<0.001)$. The level of heterogeneity was low with $\mathrm{I}^{2}=26.60$.

\section{Subgroup analyses}

Exploratory subgroup analyses are presented in Table 4. For stress $(Q=20.12, d f=1$, $p<0.001)$ and mindfulness $(Q=5.50, d f=1, p=0.019)$, significantly higher effect sizes 
were found for online MBIs with therapist guidance than for online MBIs without therapist guidance, but effect sizes did not vary based on intervention type (i.e. mindfulness or ACT) or population (i.e. healthy, psychological symptoms or physical symptoms). For depression, anxiety and well-being, no significant differences between subgroups were found.

Table 4 • Subgroup analyses (including outliers).

\begin{tabular}{|c|c|c|c|c|c|c|c|}
\hline $\begin{array}{l}\text { Outcome } \\
\text { measure }\end{array}$ & Criterion & Subgroup & $\mathrm{N}_{\text {comp }}$ & Hedge's g & $95 \% \mathrm{CI}$ & $\mathrm{I}^{2}$ & $\mathbf{Z}$ \\
\hline \multirow[t]{7}{*}{ Depression } & Intervention type & Mindfulness & 7 & 0.21 & 0.01 to 0.42 & 68.44 & $2.01^{*}$ \\
\hline & & $\mathrm{ACT}$ & 5 & 0.40 & 0.15 to 0.66 & o & $3.10^{* *}$ \\
\hline & Guidance & With & 7 & 0.29 & 0.06 to 0.53 & 57.33 & $2.48^{*}$ \\
\hline & & Without & 5 & 0.29 & 0.03 to 0.55 & 66.12 & $2.18^{*}$ \\
\hline & Population & Healthy & 4 & 0.21 & -0.07 to 0.50 & 0 & 1.48 \\
\hline & & Psychological symptoms & 3 & 0.41 & 0.07 to 0.76 & 89.51 & $2.35^{*}$ \\
\hline & & Physical symptoms & 5 & 0.29 & 0.01 to 0.56 & o & $2.05^{*}$ \\
\hline \multirow[t]{7}{*}{ Anxiety } & Intervention type & Mindfulness & 6 & 0.11 & -0.10 to 0.31 & 60.55 & 1.01 \\
\hline & & $\mathrm{ACT}$ & 5 & 0.37 & 0.13 to 0.60 & 12.43 & $3.07^{\star *}$ \\
\hline & Guidance & With & 6 & 0.26 & 0.02 to 0.50 & 50.47 & $2.09^{*}$ \\
\hline & & Without & 5 & 0.19 & -0.06 to 0.43 & 65.73 & 1.46 \\
\hline & Population & Healthy & 3 & 0.10 & -0.19 to 0.39 & o & 0.69 \\
\hline & & Psychological symptoms & 3 & 0.41 & 0.09 to 0.72 & 81.11 & $2.52^{\star}$ \\
\hline & & Physical symptoms & 5 & 0.19 & -0.06 to 0.45 & 10.97 & 1.49 \\
\hline \multirow[t]{7}{*}{ Stress } & Intervention type & Mindfulness & 9 & 0.54 & 0.27 to 0.82 & 85.18 & $3.86^{\star \star *}$ \\
\hline & & $\mathrm{ACT}$ & 2 & 0.34 & 0.28 to 0.96 & 61.22 & 1.08 \\
\hline & Guidance & With & 5 & 0.89 & 0.65 to 1.12 & 75.96 & $7.41^{* * *}$ \\
\hline & & Without & 6 & 0.19 & -0.01 to $0.3^{8}$ & o & 1.88 \\
\hline & Population & Healthy & 9 & 0.47 & 0.20 to 0.73 & 85.06 & $3.41^{* *}$ \\
\hline & & Psychological symptoms & o & - & - & - & - \\
\hline & & Physical symptoms & 2 & 0.73 & 0.12 to 1.35 & o & $2.33^{*}$ \\
\hline \multirow[t]{7}{*}{ Well-being } & Intervention type & Mindfulness & 5 & 0.28 & 0.09 to 0.48 & 36.05 & $2.84^{\star \star}$ \\
\hline & & $\mathrm{ACT}$ & 4 & 0.17 & -0.06 to 0.40 & 40.59 & 1.42 \\
\hline & Guidance & With & 5 & 0.15 & -0.05 to 0.36 & 23.05 & 1.47 \\
\hline & & Without & 4 & 0.31 & 0.11 to 0.52 & 45.09 & $3.02^{* \star}$ \\
\hline & Population & Healthy & 2 & 0.19 & -0.02 to 0.41 & $\circ$ & 1.77 \\
\hline & & Psychological symptoms & 3 & 0.43 & 0.20 to 0.66 & 56.87 & $3.61^{* * *}$ \\
\hline & & Physical symptoms & 4 & $0 . n$ & -0.09 to 0.32 & o & 1.10 \\
\hline \multirow[t]{7}{*}{ Mindfulness } & Intervention type & Mindfulness & 10 & 0.31 & 0.20 to 0.42 & 8.09 & $5.57^{* * *}$ \\
\hline & & ACT & 2 & 0.39 & 0.15 to 0.63 & 55.25 & $3.21^{\star *}$ \\
\hline & Guidance & With & 6 & 0.43 & 0.30 to 0.56 & 20.33 & $6.60^{* * *}$ \\
\hline & & Without & 6 & 0.22 & 0.10 to 0.34 & $\circ$ & $3.63^{* \star *}$ \\
\hline & Population & Healthy & 8 & 0.32 & 0.21 to 0.42 & 11.71 & $5.84^{* * *}$ \\
\hline & & Psychological symptoms & 1 & 0.56 & 0.24 to 0.87 & o & $3.46^{* *}$ \\
\hline & & Physical symptoms & 3 & 0.23 & 0.01 to 0.45 & o & $2.09^{*}$ \\
\hline
\end{tabular}

Note. $\mathrm{N}_{\text {comp }}$, number of comparisons; $\mathrm{CI}$, confidence interval. ${ }^{*} \mathrm{p}<0.05,{ }^{* \star} \mathrm{p}<0.01,{ }^{* * *} \mathrm{p}<0.001$. 


\section{Meta-regression analysis}

Using meta-regression analysis, we found no evidence that effect sizes were moderated by study quality. For stress, the number of sessions had a significant positive influence on the effect size, with more sessions resulting in higher effect sizes. This was found when we included the outlier (slope: $0.10, Z=2.22, p=0.026$ ), but not when we excluded the outlier (slope: $0.04, Z=0.78, p=0.43$ ).

\section{Publication bias}

Some indication for publication bias was found. For anxiety, stress and well-being, funnel plots were somewhat skewed in favour of studies with a positive outcome. Furthermore, the fail-safe $\mathrm{N}$ indicated that the findings for depression, stress and mindfulness were robust, whereas the fail-safe numbers for anxiety (33) and wellbeing (28) were lower than required (respectively 65 and 55 ).

When omitting either outliers or low quality studies, the findings for stress and mindfulness were still found to be robust. After removing outliers, the fail-safe N (56) for depression was slightly lower than required (6o). When low quality studies were excluded from the analysis, findings did not appear robust for depression, anxiety and well-being, with fail-safe numbers of 48,24 and 29 , respectively.

After adjusting for potential publication bias with Duval and Tweedie's trimand-fill procedure, the effect sizes for depression, anxiety, stress, well-being and mindfulness remained the same. However, for depression, four studies were imputed after removal of outliers and the adjusted effect size was $g=0.18$ (95\% CI: 0.04 to 0.31). When only studies of medium or high quality were included in the analysis, two studies were imputed for stress and the effect size was adjusted to $g=0.30$ ( $95 \% \mathrm{CI}$ : 0.10 to 0.50$)$.

\section{Discussion}

\section{Main findings}

The aim of this explorative meta-analysis was to estimate the overall effects of online MBIs on depression, anxiety, stress, well-being (primary outcomes) and mindfulness (secondary outcome) compared to controls. When all studies were taken into account, we found small but significant effect sizes for depression, anxiety, well-being and mindfulness, and a significant moderate effect size for stress. Based on the fail-safe $\mathrm{N}$, the effects on depression, stress and mindfulness appear robust.

This meta-analysis shows the most promising findings for stress. The observed effect of online MBIs on stress, including the outlier, is comparable to the effect size found for traditional MBSR and MBCT $(d=0.51)$ as found in a recent systematic 
review and meta-analysis of systematic reviews of RCTs (Gotink et al., 2015). The fact that a considerably greater beneficial impact on stress was observed, relative to the other outcomes, can be explained as the majority of studies that administered a stress outcome measure employed MBSR (8/11), which was originally developed for reducing stress in people with chronic pain (Kabat-Zinn, 1982). However, the observed effect size for stress dropped from 0.51 to 0.39 after removal of one extreme positive outlier (Wolever et al., 2012), suggesting that the effect on stress may be somewhat overestimated. One potential explanation for the divergent findings of Wolever et al. (2012) is that the intervention duration in this particular study was relatively long (12 sessions) compared to the other studies (ranging from 2 to 8 sessions). We found a moderating effect of the number of sessions on the effectiveness of online MBIs in reducing stress, although this effect seemed to be driven by the aforementioned outlier (Wolever et al., 2012). Because only one study that evaluated an online MBI with 12 sessions was included in our meta-analysis, no definite conclusions can be drawn. Moreover, the study quality of Wolever et al. (2012) was low.

Contrary to the literature, which has demonstrated that online psychotherapeutic interventions are equally effective as face-to-face interventions (Barak, Hen, Boniel-Nissim, \& Shapira, 2008), the effect sizes for depression and anxiety in this meta-analysis were in general lower than the medium to large effect sizes found for face-to-face MBIs in previous research (e.g. Abbott et al., 2014; Cavanagh et al., 2014; Gotink et al., 2015; Hofmann et al., 2010; Khoury et al., 2015; Piet, Wurtzen, \& Zachariae, 2012; Vøllestad et al., 2012; Zainal et al., 2013). These findings may suggest that online MBIs are, as yet, not equally effective as traditional face-to-face MBIs in reducing depression and anxiety. Nevertheless, drawing any conclusions based on these findings would be premature since only a relatively small number of trials addressing the effectiveness of online MBIs on depression and anxiety could be included in the present meta-analysis.

Moreover, considerable variability existed across the studies, e.g. in terms of study population. It is possible that particular subgroups may benefit more from online delivered MBIs than other groups. For instance, a meta-analysis of Barak et al. (2008) showed that Internet-based psychotherapeutic interventions are more suitable for individuals with psychological symptoms than for individuals with physical symptoms. Although we did not find strong evidence for this notion, effect sizes appeared to be larger for populations with psychological symptoms (e.g. depression, anxiety) than for healthy populations or populations with physical symptoms (e.g. chronic pain) on all outcome measures, except for stress (for stress, comparisons were only possible for healthy populations and populations with physical symptoms). However, these differences did not reach statistical significance, possibly due to the small number of studies per subgroup. Since about half of the included 
studies ( $n=7)$ were conducted in healthy samples (e.g. students and employees), the effectiveness of online MBIs in alleviating depression and anxiety might be underestimated. Healthy populations are likely to have lower baseline scores on psychological symptoms, such as depression and anxiety, leading to less room for improvement compared to clinical populations. In other words, the small effect sizes for depression and anxiety may be attributed to a floor effect.

Another possible explanation for the small effect sizes of online MBIs compared to face-to-face MBIs has to do with adherence. Non-adherence occurs when people stop using the intervention or use the intervention in a way its developers did not intend. This is a common issue in online psychological interventions and may diminish the effectiveness of an intervention (Christensen, Griffiths, \& Farrer, 2009; Donkin et al., 2011; Wangberg, Bergmo, \& Johnsen, 2008). Adherence is especially relevant in mindfulness training, as regular practise is assumed essential for developing mindfulness skills (e.g. Carmody \& Baer, 2008). In those studies included in our meta-analysis that reported adherence, adherence rates varied between $35 \%$ and $92 \%$. Due to variations in definitions and measurements of adherence along with the lack of clarity around how adherence was measured (e.g. self-reported or using log-data), we were not able to systematically study whether adherence to the intervention is significantly associated with effectiveness. Hence, we cannot rule out that non-optimal adherence may have prevented (some of) the online MBIs from reaching their full potential in terms of mental health outcomes.

This poses the question as to how adherence to online MBIs may be enhanced. Previous research indicates that providing support has a positive influence on adherence and enhances the effectiveness of online psychological interventions (Andersson \& Cuijpers, 2009; Richards \& Richardson, 2012; Spek et al., 2007). Consistently, for stress and mindfulness, significantly larger effect sizes were found for online MBIs with therapist guidance ( $g=0.89$ and $g=0.43$, respectively) than for online MBIs without therapist guidance ( $g=0.19$ and $g=0.22$, respectively) (see also Table 4). However, we did not find a significant influence of therapist guidance on depression, anxiety and well-being. In this respect, we would like to stress that the subgroup analyses were underpowered and that these findings should be interpreted with caution.

Offering therapist guidance to participants of online MBIs may thus potentially improve adherence and treatment outcomes, however, not without a few disadvantages. For instance, involvement of a therapist is costly and may restrict the scalability of the intervention. These barriers may be overcome by using automated support instead of human support. Examples of automated support, which may be helpful in the context of online MBIs, are automated text messages and personalised experience stories. Such messages and stories can address participants' possible doubts 
about the mindfulness program and/or the restlessness and sleepiness they might be experiencing, by providing suggestions on how to successfully cope with these hindrances.

Automated support has proven effective in improving adherence and effectiveness of interventions (Furmark et al., 2009; Morgan, Jorm, \& Mackinnon, 2012; Titov et al., 2010). In addition, a recent RCT (Kelders, Bohlmeijer, Pots, \& Van GemertPijnen, 2015) suggests that automated support may be as effective as human support, when enriched with persuasive e-health technologies such as text messages, interaction, tailoring and personalisation (for an overview, see Oinas-Kukkonen \& Harjumaa, 2009). In another recent study (Kelders et al., 2015), a human-supported web-based ACT intervention and an automated-supported we-based ACT intervention, both of which aimed to aid people with mild to moderate depressive symptoms, were compared to one another in terms of adherence and effectiveness. This comparison showed similar adherence rates as well as similar improvements in depression and anxiety after six months. That persuasive e-health technologies may enhance adherence and effectiveness of online interventions is also confirmed by a systematic review of adherence to web-based interventions (Kelders, Kok, Ossebaard, \& Van Gemert-Pijnen, 2012).

With respect to study quality, we found that when low quality studies were omitted from the analysis, virtually the same effects were found for each outcome measure except for stress for which the effect size dropped from 0.51 to 0.40 . However, the meta-regression analysis indicated that there was no significant relationship between the methodological quality of the studies and effect sizes for any of the outcome measures. While this finding is in line with previous meta-analyses investigating the effects of MBIs (Bohlmeijer et al., 2010; Hofmann et al., 2010; Klainin-Yobas et al., 2012; Powers, Zum Vörde Sive Vörding, \& Emmelkamp, 2009; Strauss et al., 2014; Veehof et al., 2011), there are also meta-analyses which indicate that higher quality studies yield smaller effect sizes (A-Tjak et al., 2015; Khoury et al., 2013). Nonetheless, we recommend researchers conducting RCTs on online MBIs to comply with the criteria for designing high quality trials, in order to build a body of sound scientific knowledge on the effectiveness of online MBIs.

\section{Limitations and directions for future research}

This meta-analysis had several limitations. First, despite the growing empirical literature on the effectiveness of online MBIs in terms of mental health outcomes, we were only able to include a relatively small number of RCTs in our meta-analysis. Second, the effect sizes of the included studies varied considerably per outcome, which may be explained by differences in study characteristics, such as population, intervention type (e.g. ACT, MBSR or MBCT), and outcome measures. The small 
number of studies and substantial variability across studies warrants caution in interpreting and generalising the observed effect sizes. Third, although we conducted several subgroup analyses in order to explore potential moderators of the effects of online MBIs, it must be acknowledged that these analyses were underpowered and that the findings should be interpreted tentatively. Fourth, given the small number of studies and the fact that only two studies concerned MBCT, it was not possible to conduct separate meta-analyses for $A C T, M B S R$ and $M B C T$, respectively. These interventions use somewhat different approaches, for example, MBCT and ACT incorporate elements of cognitive behavioural therapy as opposed to MBSR. Furthermore, ACT uses mindfulness techniques, but does not require meditation, whereas MBCT and MBSR are meditation-based. Hence, the interventions might not be equally effective. Finally, it was not possible to conduct a meta-analysis of the long-term effects of online MBIs because of the high variability in follow-up periods (ranging from 12 weeks to 1 year). This is considered important, because multiple trials have shown that effects of online MBIs are maintained up to one year after baseline (e.g. Hesser et al., 2012; Pots et al., 2015).

Given the widespread attention for mindfulness and the potential value of online MBIs for clinical practice, additional research to establish the beneficial effects of online MBIs and to gain insight in their moderators of effectiveness is warranted. Future research might focus on a number of specific areas, including: 1) testing whether the observed beneficial effects of online MBIs on depression, anxiety, stress, well-being and mindfulness are maintained over time; 2) assessing the clinical utility of online MBIs across various subgroups (e.g. psychological versus somatic illnesses) and in various (clinical) populations; and 3 ) identifying moderators of the effects of online MBIs (e.g. type of intervention: ACT, MBSR or MBCT; delivery mode: smartphone versus computer).

In addition, we encourage researchers in the field to take into account study quality criteria. Although most studies were of satisfactory quality, only two studies (Levin, Pistorello, Seeley, \& Hayes, 2014; Morledge et al., 2013) could be classified as high quality. In particular, the description of withdrawals/drop-outs (Criterion 3 ) and the sample size being based on an adequate power analysis (Criterion 5 ) were often not adequately addressed. Finally, we strongly recommend researchers to not only report on study dropouts, but to address adherence to the intervention as well (e.g. number of sessions completed and length of time practiced). Given the dose-response relationship that has been found for the use of online interventions (Christensen et al., 2009; Donkin et al., 2011; Wangberg et al., 2008), adherence seems an important factor to take into account when considering the effectiveness of online MBIs. This finding is corroborated in the study of Trompetter et al. (2014) which yielded significantly greater gains for adherers than for non-adherers. 


\section{Conclusions and implications}

To our knowledge, this is the first meta-analysis that evaluates the specific effects of online MBIs on mental health and well-being. It has been argued that online interventions in the context of public mental health are a promising strategy to alleviate psychological symptomatology and reduce the prevalence of severe mental health problems (Barak et al., 2008; Fledderus, Bohlmeijer, Pieterse, \& Schreurs, 2012; Pots et al., 2015; Ybarra \& Eaton, 2005). Our findings, in turn, contribute to a better understanding of the effectiveness of online MBIs. Although research exploring the effectiveness of online MBIs is still in its infancy, we conclude that there is emerging evidence that online MBIs have the potential to improve mental health outcomes, most notably stress.

We found small effects for most outcomes (i.e. depression, anxiety, well-being, and mindfulness). Nonetheless, the wide reach and low cost of online MBIs may facilitate improved mental health and well-being in many people (with psychological distress). Online MBIs may be used in various manners and for various purposes. For instance, online MBIs might be an acceptable and useful alternative for people who may benefit from cultivating their mindfulness skills, but cannot be reached with traditional (individual or group-based) face-to-face formats (e.g. Wahbeh et al., 2014). In addition, online MBIs may be offered to individuals who are on a waitlist to receive a face-to-face MBI. Furthermore, online MBIs may be integrated in other (online) psychotherapeutic interventions (e.g. cognitive behavioural therapy) aimed at decreasing distress and/or enhancing well-being (e.g. Bohlmeijer et al., 2010; Veehof et al., 2011). 


\section{References}

A-Tjak, J. G. L., Davis, M. L., Morina, N., Powers, M. B., Smits, J. A. J., \& Emmelkamp, P. M. G. (2015). A meta-analysis of the efficacy of acceptance and commitment therapy for clinically relevant mental and physical health problems. Psychotherapy and Psychosomatics, 84, 30-36.

Abbott, R. A., Whear, R., Rodgers, L. R., Bethel, A., Thompson Coon, J., Kuyken, W., et al. (2014). Effectiveness of mindfulness-based stress reduction and mindfulness based cognitive therapy in vascular disease: A systematic review and meta-analysis of randomised controlled trials. Journal of Psychosomatic Research, 76, 341-351.

Aikens, K. A., Astin, J., Pelletier, K. R., Levanovich, K., Baase, C. M., Park, Y. Y., et al. (2014). Mindfulness goes to work: Impact of an online workplace intervention. Journal of Occupational and Environmental Medicine, 56, 721-731.

Andersson, G., \& Cuijpers, P. (2009). Internet-based and other computerized psychological treatments for adult depression: A meta-analysis. Cognitive Behaviour Therapy, 38, 196-205.

Andersson, G., \& Titov, N. (2014). Advantages and limitations of internet-based interventions for common mental disorders. World Psychiatry, 13, 4-11.

Baer, R. A. (2003). Mindfulness training as a clinical intervention: A conceptual and empirical review. Clinical Psychology: Science and Practice, 10, 125-143.

Barak, A., Hen, L., Boniel-Nissim, M., \& Shapira, N. (2008). A comprehensive review and a meta-analysis of the effectiveness of internet-based psychotherapeutic interventions. Journal of Technology in Human Services, 26, 109-160.

Barak, A., Klein, B., \& Proudfoot, J. G. (2009). Defining internet-supported therapeutic interventions. Annals of Behavioral Medicine, 38, 4-17.

Bishop, S. R. (2002). What do we really know about mindfulness-based stress reduction? Psychosomatic Medicine, 64, 71-84.

Bishop, S. R., Lau, M., Shapiro, S., Carlson, L., Anderson, N. D., Carmody, J., et al. (2004). Mindfulness: A proposed operational definition. Clinical Psychology: Science and Practice, 17, 230-241.

Boettcher, J., Åström, V., Påhlsson, D., Schenström, O., Andersson, G., \& Carlbring, P. (2014). Internet-based mindfulness treatment for anxiety disorders: A randomized controlled trial. Behavior Therapy, 45, 241-253.

Bohlmeijer, E., Prenger, R., Taal, E., \& Cuijpers, P. (2010). The effects of mindfulness-based stress reduction therapy on mental health of adults with a chronic medical disease: A meta-analysis. Journal of Psychosomatic Research, 68, 539-544.

Buhrman, M., Skoglund, A., Husell, J., Bergstrom, K., Gordh, T., Hursti, T., et al. (2013). Guided internetdelivered acceptance and commitment therapy for chronic pain patients: A randomized controlled trial. Behaviour Research and Therapy, 51, 307-315.

Carmody, J., \& Baer, R. A. (2008). Relationships between mindfulness practice and levels of mindfulness, medical and psychological symptoms and well-being in a mindfulness-based stress reduction program. Journal of Behavioral Medicine, 31, 23-33.

Cavanagh, K., Strauss, C., Cicconi, F., Griffiths, N., Wyper, A., \& Jones, F. (2013). A randomised controlled trial of a brief online mindfulness-based intervention. Behaviour Resesearch and Therapy, 51, 573-578.

Cavanagh, K., Strauss, C., Forder, L., \& Jones, F. (2014). Can mindfulness and acceptance be learnt by self-help? A systematic review and meta-analysis of mindfulness and acceptance-based self-help interventions. Clinical Psychology Review, 34, 118-129.

Chiesa, A., \& Serretti, A. (2009). Mindfulness-based stress reduction for stress management in healthy people: A review and meta-analysis. Journal of Alternative and Complementary Medicine, 15, 593-600.

Chiesa, A., \& Serretti, A. (2011). Mindfulness-based cognitive therapy for psychiatric disorders: A systematic review and meta-analysis. Psychiatry Research, 187, 441-453. 
Christensen, H., Griffiths, K. M., \& Farrer, L. (2009). Adherence in internet interventions for anxiety and depression. Journal of Medical Internet Research, 11, e13.

Cramer, H., Lauche, R., Paul, A., \& Dobos, G. (2012). Mindfulness-based stress reduction for breast cance: A systematic review and meta-analysis. Current Oncology, 19, e343-352.

Cuijpers, P., Marks, I. M., van Straten, A., Cavanagh, K., Gega, L., \& Andersson, G. (2009). Computer-aided psychotherapy for anxiety disorders: A meta-analytic review. Cognitive Behaviour Therapy, 38, 66-82.

De Vibe, M., Bjørndal, A., Tipton, E., Hammerstrøm, K. T., \& Kowalski, K. (2012). Mindfulness based stress reduction (mbsr) for improving health, quality of life, and social functioning in adults. Campbell Systematic Reviews, 3.

Donkin, L., Christensen, H., Naismith, S. L., Neal, B., Hickie, I. B., \& Glozier, N. (2011). A systematic review of the impact of adherence on the effectiveness of e-therapies. Journal of Medical Internet Research, 13, e52.

Dowd, H., Hogan, M. J., McGuire, B. E., Davis, M. C., Sarma, K. M., Fish, R. A., et al. (2015). Comparison of an online mindfulness-based cognitive therapy intervention with online pain management psychoeducation: A randomized controlled study. Clinical Journal of Pain, 31, 517-527.

Duval, S., \& Tweedie, R. (2000). Trim and fill: A simple funnel-plot-based method of testing and adjusting for publication bias in meta-analysis. Biometrics, 56, 455-463.

Egger, M., Davey Smith, G., Schneider, M., \& Minder, C. (1997). Bias in meta-analysis detected by a simple, graphical test. BMJ, 315, 629-634.

Feigenbaum, J. (2007). Dialectical behaviour therapy: An increasing evidence base. Journal of Mental Health, 16, 51-68.

Fledderus, M., Bohlmeijer, E. T., Pieterse, M. E., \& Schreurs, K. M. (2012). Acceptance and commitment therapy as guided self-help for psychological distress and positive mental health: $A$ randomized controlled trial. Psychological Medicine, 42, 485-495.

Furmark, T., Carlbring, P., Hedman, E., Sonnenstein, A., Clevberger, P., Bohman, B., et al. (2009). Guided and unguided self-help for social anxiety disorder: Randomised controlled trial. British Journal of Psychiatry, 195, 440-447.

Glück, T. M., \& Maercker, A. (2011). A randomized controlled pilot study of a brief web-based mindfulness training. BMC Psychiatry, 11, 175.

Gotink, R. A., Chu, P., Busschbach, J. J., Benson, H., Fricchione, G. L., \& Hunink, M. G. (2015). Standardised mindfulness-based interventions in healthcare: An overview of systematic reviews and meta-analyses of rcts. PLoS One, 10, eo124344.

Hayes, S. C., Luoma, J. B., Bond, F. W., Masuda, A., \& Lillis, J. (2006). Acceptance and commitment therapy: Model, processes and outcomes. Behaviour Research and Therapy, 44, 1-25.

Hayes, S. C., Strosahl, K., \& Wilson, K. G. (1999). Acceptance \& commitent therapy: An experiential approach to behavior change. New York: Guilford Press.

Hedges, L. V., \& Vevea, J. L. (1998). Fixed-and random-effects models in meta-analysis. Psychological Methods, 3, 486-504.

Hesser, H., Gustafsson, T., Lundén, C., Henrikson, O., Fattahi, K., Johnsson, E., et al. (2012). A randomized controlled trial of internet-delivered cognitive behavior therapy and acceptance and commitment therapy in the treatment of tinnitus. Journal of Consulting and Clinical Psychology, $80,649-661$.

Higgins, J. P., \& Thompson, S. G. (2002). Quantifying heterogeneity in a meta-analysis. Statistics in Medicine, 21, 1539-1558.

Higgins, J. P. T., Altman, D. G., \& Sterne, J. A. C. (2011). Assessing risk of bias in included studies. In J. P. T. Higgins \& S. Green (Eds.), Cochrane Handbook for Systematic Reviews of Interventions (5.1.0 ed.). Available from www.cochrane-handbook.org.

Hofmann, S. G., Sawyer, A. T., Witt, A. A., \& Oh, D. (2010). The effect of mindfulness-based therapy on anxiety and depression: A meta-analytic review. Journal of Consulting and Clinical Psychology, $78,169-183$. 
Jadad, A. R., Andrew Moore, R., Carroll, D., Jenkinson, C., Reynolds, D. J. M., Gavaghan, D. J., et al. (1996). Assessing the quality of reports of randomized clinical trials: Is blinding necessary? Controlled Clinical Trials, 17, 1-12.

Kabat-Zinn, J. (1982). An outpatient program in behavioral medicine for chronic pain patients based on the practice of mindfulness meditation: Theoretical considerations and preliminary results. General Hospital Psychiatry, 4, 33-47.

Kabat-Zinn, J. (1990). Full catastrophe living: Using the wisdom of your body and mind to face stress, pain and illness. New York: Delacorte.

Kelders, S. M., Bohlmeijer, E. T., Pots, W. T., \& Van Gemert-Pijnen, J. E. (2015). Comparing human and automated support for depression: Fractional factorial randomized controlled trial. Behaviour Research and Therapy, 72, 72-80.

Kelders, S. M., Kok, R. N., Ossebaard, H. C., \& Van Gemert-Pijnen, J. E. (2012). Persuasive system design does matter: A systematic review of adherence to web-based interventions. Journal of Medical Internet Research, 14, e152.

Keng, S. L., Smoski, M. J., \& Robins, C. J. (2011). Effects of mindfulness on psychological health: A review of empirical studies. Clinical Psychology Review, 31, 1041-1056.

Khoury, B., Lecomte, T., Fortin, G., Masse, M., Therien, P., Bouchard, V., et al. (2013). Mindfulness-based therapy: A comprehensive meta-analysis. Clinical Psychology Review, 33, 763-771.

Khoury, B., Sharma, M., Rush, S. E., \& Fournier, C. (2015). Mindfulness-based stress reduction for healthy individuals: A meta-analysis. Journal of Psychosomatic Research, 78, 519-528.

Klainin-Yobas, P., Cho, M. A., \& Creedy, D. (2012). Efficacy of mindfulness-based interventions on depressive symptoms among people with mental disorders: A meta-analysis. International Journal of Nursing Studies, 49, 109-121.

Lauche, R., Cramer, H., Dobos, G., Langhorst, J., \& Schmidt, S. (2013). A systematic review and meta-analysis of mindfulness-based stress reduction for the fibromyalgia syndrome. Journal of Psychosomatic Research, 75, 500-510.

Ledesma, D., \& Kumano, H. (2009). Mindfulness-based stress reduction and cancer: A meta-analysis. Psychooncology, 18, 571-579.

Levin, M. E., Pistorello, J., Seeley, J. R., \& Hayes, S. C. (2014). Feasibility of a prototype web-based acceptance and commitment therapy prevention program for college students. Journal of American College Health, 62, 20-30.

Linehan, M. (1993). Cognitive-behavioral treatment of borderline personality disorder. New York: Guilford Press.

Lipsey, M. W., \& Wilson, D. B. (1993). The efficacy of psychological, educational, and behavioral treatment: Confirmation from meta-analysis. American Psychologist, 48, 1181-1209.

Ly, K. H., Truschel, A., Jarl, L., Magnusson, S., Windahl, T., Johansson, R., et al. (2014). Behavioural activation versus mindfulness-based guided self-help treatment administered through a smartphone application: A randomised controlled trial. BMJ Open, 4, eoo3440.

Mak, W. W., Chan, A. T., Cheung, E. Y., Lin, C. L., \& Ngai, K. C. (2015). Enhancing web-based mindfulness training for mental health promotion with the health action process approach: Randomized controlled trial. Journal of Medical Internet Research, 17, e8.

McCarney, R. W., Schulz, J., \& Grey, A. R. (2012). Effectiveness of mindfulness-based therapies in reducing symptoms of depression: A meta-analysis. European Journal of Psychotherapy \& Counselling, 14, 279-299.

Metcalf, C. A., \& Dimidjian, S. (2014). Extensions and mechanisms of mindfulness-based cognitive therapy: A review of the evidence. Australian Psychologist, 49, 271-279.

Moher, D., Liberati, A., Tetzlaff, J., Altman, D. G., Altman, D., Antes, G., et al. (2009). Preferred reporting items for systematic reviews and meta-analyses: The prisma statement. PloS Medicine, 6, elooooog7.

Morgan, A. J., Jorm, A. F., \& Mackinnon, A. J. (2012). Email-based promotion of self-help for subthreshold depression: Mood memos randomised controlled trial. British Journal of Psychiatry, 200, 412-418. 
Morledge, T. J., Allexandre, D., Fox, E., Fu, A. Z., Higashi, M. K., Kruzikas, D. T., et al. (2013). Feasibility of an online mindfulness program for stress management - a randomized, controlled trial. Annals of Behavioral Medicine, 46, 137-148.

Oinas-Kukkonen, H., \& Harjumaa, M. (2009). Persuasive systems design: Key issues, process model, and system features. Communications of the Association for Information Systems, 24, 485-50o.

Öst, L. G. (2014). The efficacy of acceptance and commitment therapy: An updated systematic review and meta-analysis. Behaviour Research and Therapy, 61, 105-121.

Piet, J., \& Hougaard, E. (2011). The effect of mindfulness-based cognitive therapy for prevention of relapse in recurrent major depressive disorder: A systematic review and meta-analysis. Clinical Psychology Review, 31, 1032-1040.

Piet, J., Wurtzen, H., \& Zachariae, R. (2012). The effect of mindfulness-based therapy on symptoms of anxiety and depression in adult cancer patients and survivors: A systematic review and meta-analysis. Journal of Consulting and Clinical Psychology, 80, 1007-1020.

Piet, J., Würtzen, H., \&Zachariae, R. (2012). The effect of mindfulness-based therapy on symptoms of anxiety and depression in adult cancer patients and survivors: A systematic review and meta-analysis. Journal of Consulting and Clinical Psychology, 80, 1007-1020.

Pots, W. T., Fledderus, M., Meulenbeek, P. A., Ten Klooster, P. M., Schreurs, K. M., \& Bohlmeijer, E. T. (2015). Acceptance and commitment therapy as a web-based intervention for depressive symptoms: Randomised controlled trial. British Journal of Psychiatry, 208, 69-77.

Powers, M. B., Zum Vörde Sive Vörding, M. B., \& Emmelkamp, P. M. G. (2009). Acceptance and commitment therapy: A meta-analytic review. Psychotherapy and Psychosomatics, 78, 73-80.

Praissman, S. (2008). Mindfulness-based stress reduction: A literature review and clinician's guide. Journal of the American Academy of Nurse Practitioners, 20, 212-216.

Richards, D., \& Richardson, T. (2012). Computer-based psychological treatments for depression: A systematic review and meta-analysis. Clinical Psychology Review, 32, 329-342.

Rosenberg, M. S. (2005). The file-drawer problem revisited: A general weighted method for calculating fail-safe numbers in meta-analysis. Evolution, 59, 464-468.

Ruiz, F. J. (2010). A review of acceptance and commitment therapy (act) empirical evidence: Correlational, experimental psychopathology, component and outcome studies. International Journal of Psychology \& Psychological Therapy, 10, 125-162.

Segal, Z. V., Williams, J. M. G., \& Teasdale, J. D. (2002). Mindfulness-based cognitive therapy for depression: A new approach to preventing relapse. New York: The Guilford Press.

Spek, V., Cuijpers, P., Nyklicek, I., Riper, H., Keyzer, J., \& Pop, V. (2007). Internet-based cognitive behaviour therapy for symptoms of depression and anxiety: A meta-analysis. Psychological Medicine, 37, 319-328.

Sterne, J. A. C., Egger, M., \& Moher, D. (2008). Addressing reporting biases. In J. P. Higgins \& S. Green (Eds.), Cochrane handbook for systematic reviews of interventions: Cochrane book series. Chichester, UK: John Wiley \& Sons.

Strauss, C., Cavanagh, K., Oliver, A., \& Pettman, D. (2014). Mindfulness-based interventions for people diagnosed with a current episode of an anxiety or depressive disorder: A meta-analysis of randomised controlled trials. PLoS One, 9, eg6110.

Titov, N., Andrews, G., Davies, M., McIntyre, K., Robinson, E., \& Solley, K. (2010). Internet treatment for depression: A randomized controlled trial comparing clinician vs. Technician assistance. PLoS One, 5, elog39.

Trompetter, H. R., Bohlmeijer, E. T., Veehof, M. M., \& Schreurs, K. M. G. (2014). Internet-based guided self-help intervention for chronic pain based on acceptance and commitment therapy: A randomized controlled trial. Journal of Behavioral Medicine, 38, 66-80.

Veehof, M. M., Oskam, M. J., Schreurs, K. M., \& Bohlmeijer, E. T. (2011). Acceptance-based interventions for the treatment of chronic pain: A systematic review and meta-analysis. Pain, 152, 533-542.

Visted, E., Vøllestad, J., Nielsen, M. B., \& Nielsen, G. H. (2014). The impact of group-based mindfulness training on self-reported mindfulness: A systematic review and meta-analysis. Mindfulness, 6, 501-522. 
Vøllestad, J., Nielsen, M. B., \& Nielsen, G. H. (2012). Mindfulness- and acceptance-based interventions for anxiety disorders: A systematic review and meta-analysis. British Journal of Clinical Psychology, 51, 239-260.

Wahbeh, H., Svalina, M. N., \& Oken, B. S. (2014). Group, one-on-one, or internet? Preferences for mindfulness meditation delivery format and their predictors. Open Medicine Journal, 1, 66-74.

Wangberg, S. C., Bergmo, T. S., \& Johnsen, J.-A. K. (2008). Adherence in internet-based interventions. Patient Preference and Adherence, 2, 57-65.

Wolever, R. Q., Bobinet, K. J., McCabe, K., Mackenzie, E. R., Fekete, E., Kusnick, C. A., et al. (2012). Effective and viable mind-body stress reduction in the workplace: A randomized controlled trial. Journal of Occupational Health Psychology, 17, 246-258.

Ybarra, M. L., \& Eaton, W. W. (2005). Internet-based mental health interventions. Mental Health Services Research, 7, 75-87.

Zainal, N. Z., Booth, S., \& Huppert, F. A. (2013). The efficacy of mindfulness-based stress reduction on mental health of breast cancer patients: A meta-analysis. Psychooncology, 22, 1457-1465.

Zernicke, K. A., Campbell, T. S., Speca, M., McCabe-Ruff, K., Flowers, S., \& Carlson, L. E. (2014). A randomized wait-list controlled trial of feasibility and efficacy of an online mindfulness-based cancer recovery program: The etherapy for cancer applying mindfulness trial. Psychosomatic Medicine, 76, 257-267. 


\title{
Appendix: Full electronic search strategies
}

\author{
Search strategy: PsycINFO (EBSCO) \\ \#1 TI (mindful* OR acceptance OR meditation) OR AB (mindful* OR acceptance OR meditation) \\ OR KW (mindful* OR acceptance OR meditation) \\ \#2 DE "Mindfulness" OR DE "Acceptance and Commitment Therapy" OR DE "Meditation" \\ \#3 TI (intervention* OR therap* OR treatment* OR program*) OR AB (intervention* OR therap* \\ OR treatment* OR program*) OR KW (intervention* OR therap* OR treatment* OR program*) \\ \#4 TI (online OR e-health OR Internet* OR web* OR computer* OR app OR apps) OR AB (online \\ OR e-health OR Internet* OR web* OR computer* OR app OR apps) OR KW (online OR \\ e-health OR Internet* OR web* OR computer* OR app OR apps) \\ \#5 DE "Mobile Devices" OR DE "Computers" \\ \#6 \#6: DE "Online Therapy" OR DE "Computer Assisted Therapy" \\ \#7 \#7: TI (random* OR trial OR RCT OR control*) OR AB (random* OR trial OR RCT OR control*) \\ OR KW (random* OR trial OR RCT OR control*) \\ \#8 DE "Clinical trials" OR DE "Treatment Effectiveness Evaluation" \\ \#9 \#1 OR \#2 \\ $\# 10 \quad \# 4 \mathrm{OR} \# 5$ \\ $\# 11 \quad \#_{3} \mathrm{AND} \#_{10}$ \\ $\# 12 \quad \# 11$ OR \#6 \\ $\# 13 \quad \# 7$ OR \#8 \\ \#14 \#9 AND \#12 AND \#13 \\ \#15 \#14 (Filters: English, journal article)
}

Search strategy: Web of Science

\#1 $\quad$ TS $=$ (mindful* OR acceptance OR meditation)

\#2 TS=(intervention* OR therap* OR treatment* OR program*)

\#3 TS=(online OR e-health OR Internet* OR web* OR computer* OR app OR apps)

\#4 TS=(random* OR trial OR RCT OR control*)

$\#_{5} \quad \#_{1}$ AND \# 2 AND \# 3 AND \#4

\#6 \#5 (Filters: English, journal article)

\section{Search strategy: PubMed}

\#1 mindful*[tiab] OR acceptance[tiab] OR meditation[tiab]

\#2 "Mindfulness"[Mesh] OR "Acceptance and Commitment Therapy"[Mesh] OR "Meditation"[Mesh]

\#3 intervention*[tiab] OR therap*[tiab] OR treatment*[tiab] OR program*[tiab]

\#4 online[tiab] OR e-health[tiab] OR Internet*[tiab] OR web*[tiab] OR computer*[tiab] OR app[tiab] OR apps[tiab]

\#5 "Computers"[Mesh] OR "Mobile Applications"[Mesh]

\#6 random*[tiab] OR trial[tiab] OR RCT[tiab] OR control*[tiab]

\#7 "Controlled Clinical Trial"[Mesh] OR "Randomized Controlled Trial"[Mesh] OR "Random Allocation"[Mesh] OR "Treatment Outcome"[Mesh]

$\# 8 \quad \#_{1} \mathrm{OR} \# 2$

$\# 9 \quad \#_{4} \mathrm{OR} \# 5$

$\# 10 \quad \# 6 \mathrm{OR} \# 7$

\#11 \#3 AND \#8 AND \#9 AND \#10

\#12 \#11 (Filters: English, journal article)

Search strategy: www.clinicaltrialsregister.eu

\#1 (mindfulness OR acceptance OR meditation) 
\#2 (online OR Internet OR e-health OR computer OR web OR app)

\#3 (intervention OR therapy OR treatment OR program)

\#4 \#1 AND \# 2 AND \#3

Search strategy: www.isrctn.com

\#1 (mindfulness OR acceptance OR meditation)

\#2 (online OR Internet OR e-health OR computer OR web-based OR app)

\#3 (intervention OR therapy OR treatment OR program)

\#4 \#1 AND \# 2 AND \#3

Search strategy: www.clinicaltrials.gov

\#1 (mindfulness OR acceptance OR meditation)

\#2 (online OR Internet OR e-health OR computer OR web)

\#3 (RCT OR random OR control)

\#4 \# AND \#2 AND \#3

\#5 \#4 (Filters: closed studies, interventional studies, adult, senior) 


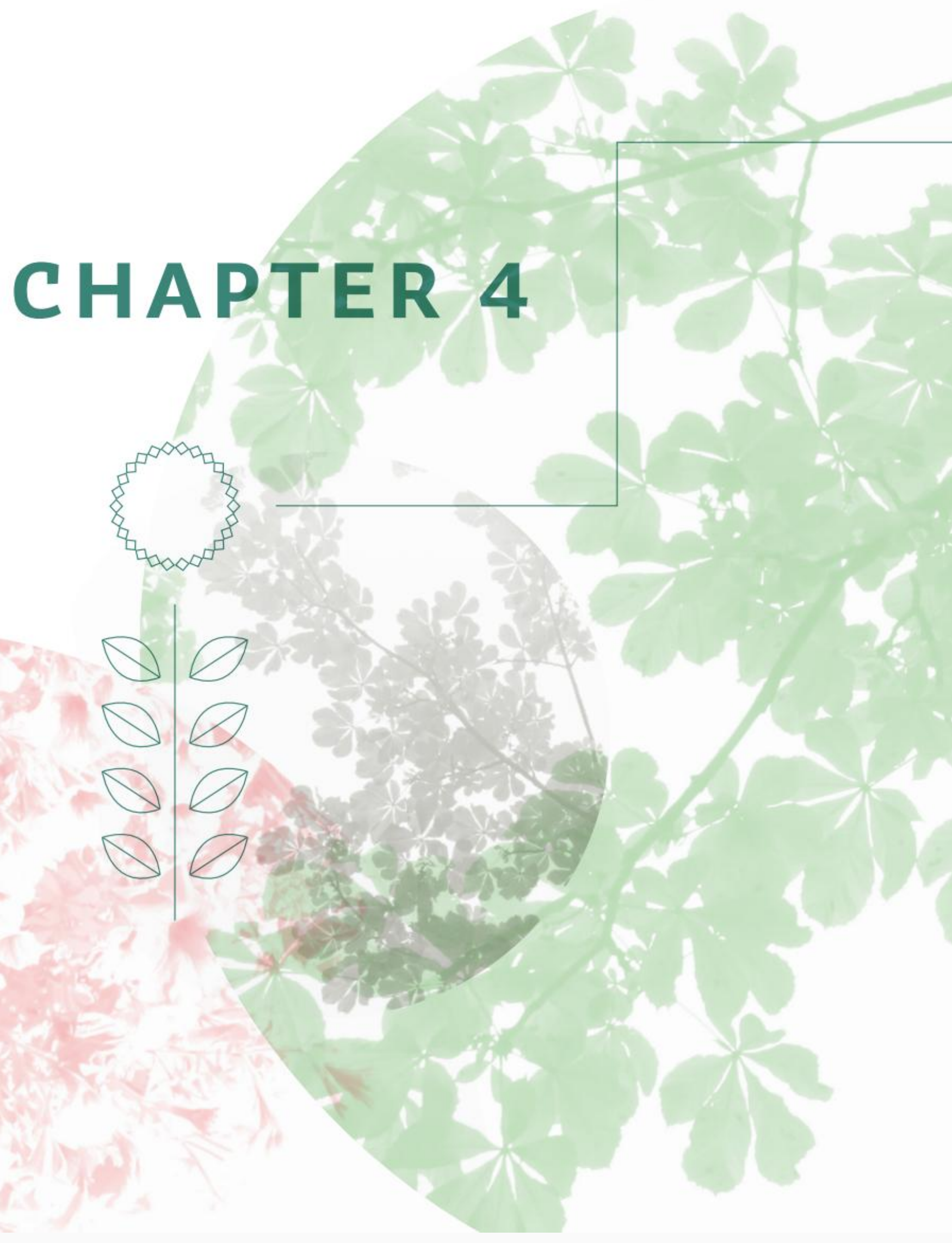




\section{Development of a web-based intervention for the indicated prevention of depression}

Kelders, S.M., Pots, W.T.M, Oskam, M. J., Bohlmeijer, E.T. \& van Gemert-Pijnen, J. E. W. C. (2013). Development of a web-based intervention for the indicated prevention of depression.

BMC Medical Informatics and Decision Making 13, 1-11

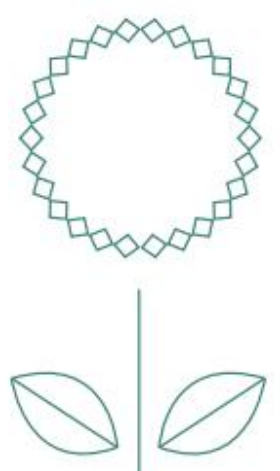




\section{Abstract}

To reduce the large public health burden of the high prevalence of depression, preventive interventions targeted at people at risk are essential and can be costeffective. Web-based interventions are able to provide this care, but there is no agreement on how to best develop these applications and often the technology is seen as a given. This seems to be one of the main reasons that web-based interventions do not reach their full potential. The current study describes the development of a web-based intervention for the indicated prevention of depression, employing the CeHRes (Center for eHealth Research and Disease Management) roadmap.The goals are to create a user-friendly application which fits the values of the stakeholders and to evaluate the process of development. The employed methods are a literature scan and discussion in the contextual inquiry; interviews, rapid prototyping and a requirement session in the value specification stage; and user-based usability evaluation, expert-based usability inspection and a requirement session in the design stage. The contextual inquiry indicated that there is a need for easily accessible interventions for the indicated prevention of depression and web-based interventions are seen as potentially meeting this need. The value specification stage yielded expected needs of potential participants, comments on the usefulness of the proposed features and comments on two proposed designs of the web-based intervention. The design stage yielded valuable comments on the system, content and service of the web-based intervention. Overall, we found that by developing the technology, we successfully (re)designed the system, content and service of the web-based intervention to match the values of stakeholders. This study has shown the importance of a structured development process of a web-based intervention for the indicated prevention of depression because: 1) it allows the development team to clarify the needs, that have to be met for the intervention to be of use to the target audience; and 2) it yields feedback on the design of the application that is broader than colour and buttons, but encompasses comments on the quality of the service that the application offers. 


\section{Background}

To reduce the large public health burden of the high prevalence of depression, preventive interventions targeted at people at risk (indicated prevention) are essential and can be cost-effective (Andrews et al., 2004, Cuijpers et al., 2008). However, recruiting participants for interventions to prevent the onset of depressive disorders is quite a challenge (Cuijpers et al., 2010). Developing and implementing web-based interventions provide an opportunity to overcome this challenge by tackling the reasons for the low participation rates (Cuijpers et al., 2010; Postel et al., 2010; Riper et al., 2007). For example, web-based interventions can decrease the stigma associated with a (mental) health condition by providing a certain degree of anonymity (Griffiths et al., 2006; Postel et al., 2011). Advantages of web-based interventions can be seen not only in the broader reach, but also in increasing convenience for the users, the opportunity to provide information in an interactive and timely manner and cost-effectiveness (Eysenbach, 2001; Griffiths et al., 2006; Tate et al., 2009). Meta-analyses on web-based interventions for the treatment and indicated prevention of mental health complaints have shown that these interventions, on average, are effective in reducing the severity of mental health complaints (Andrews et al., 2010; Barak et al., 2008).

However, not all web-based interventions show these positive effects. In many cases the effects are less than expected and the implementation of these interventions in regular care is lacking (Black et al., 2011; Christensen et al., 2011; Riper et al., 2007; van Gemert-Pijnen et al., 2011). It seems that the problem of non-adherence, i.e. participants not following the intervention protocol, is one of the issues behind the lacking effect (Christensen et al., 2009; Eysenbach, 2005; Kelders et al., 2012). Studies have shown that better adherence is associated with better (clinical) outcomes of an intervention (see for a systematic review Donkin et al., 2011). The reasons behind non-adherence are still unclear, although there are many proposed reasons. Important proposed reasons are: issues with the usability of the application (Eysenbach, 2005); issues regarding the attunement of the goals of the technology with the aims of the participants (Kelders et al., 2011; van Gemert-Pijnen et al., 2011); and implementation issues as the lack of clarity in the costs-benefit structure and integrating the technology in usual care and daily life (Nijland, 2011; van Limburg et al., 2011).

Web-based applications are developed at a startling rate, but there is no scientifically underpinned agreement on how to best develop these applications (Pagliari, 2007). Many web-based interventions seem to be designed ad hoc; there is a presumed problem for which technology is supposed to be the solution, or the technology is used as a starting point and is developed because of the technological possibility, not because of the needs of the target group. In many cases, the content 
of these web-based interventions has been the subject of research and consists of evidence-based therapies, but when creating a web-based intervention based on this content, the technology is seen as a given. This ad hoc design and a lack of a holistic overview, in which the human and technological context is given a prominent place, seems to be one of the main reasons that web-based interventions do not reach their full potential in terms of adherence and outcomes (Pagliari, 2007; van Gemert-Pijnen et al., 2011; Yusof et al., 2008).

In a recent viewpoint paper (van Gemert-Pijnen et al., 2011) a holistic framework to improve the uptake and impact of eHealth technologies was proposed. This framework is aimed at overcoming the problems described in the earlier paragraphs. The framework is based on persuasive technology theories, human centred design approaches and business modelling. Persuasive technology refers to the capacity of technology to influence behaviour and is used in eHealth research to understand the role of technology in changing behaviour (Fogg, 2003; Oinas-Kukkonen and Harjumaa, 2009). Human centred design advocates the systematic, continuous consultation of potential users during the whole design process (Gould and Lewis, 1985) and has been shown to have a positive effect especially on user satisfaction and on fitting to user needs (Kujala, 2003). Business modelling stems from commercial strategic management (Bartholomew, 2011) and focuses on value creation with stakeholders. In eHealth this approach can be used to make the development of eHealth technology value-driven, i.e. creating technology that matches the values of and makes sense to the different stakeholders (van Limburg et al., 2011). The framework includes the CeHres (Center for eHealth Research and Disease Management) roadmap for the development of eHealth technologies (Figure 1). This development strategy prescribes a structured development of not only the content of an intervention, but also of the system (or technology) and the service (including the long term implementation). Differences with other development approaches (e.g. Intervention Mapping (Bartholomew, 2011) and the RE-AIM framework (Glasgow et al., 1999; Kinzie et al., 2002)) lie in the specific focus of the development of the technology and the fit between technology and the content; and in the attention for implementation during the whole process of development. In this paper, we describe a study into the development of a web-based intervention for the indicated prevention of depression, focusing on the first steps of development according to the CeHRes roadmap: contextual inquiry, value specification and design. Important to note is that, although the roadmap describes separate activities with a summative evaluation as the last activity, the roadmap is iterative in nature and prescribes continuous evaluation. This is done through formative cycles that reflect on and inform the development process and check whether the intended goals of the technology are reached. According to the roadmap, each development process should have multidisciplinary project manage- 
ment that facilitates between the creators and the users of the technology. In this study, the project management team consisted of researchers, a clinical psychologist working at the University and at a mental health institute, a developer of the course "Living to the Full" (which is the basis for the content of the web-based intervention) who is working at the University, a web-designer and technical programmers.

In the contextual inquiry, information is gathered from the intended users and their environment to see whether there is a need for technology and how this technology might fit into the daily routines of the intended users. In this study, this is done by conducting a literature scan, combined with discussions with the project management team.

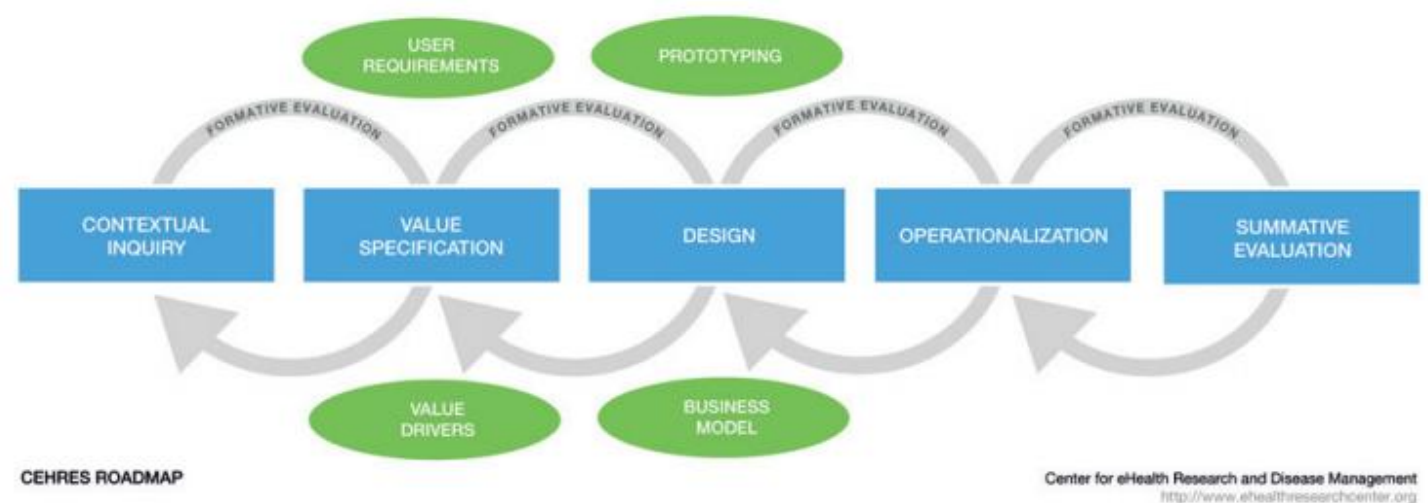

Figure 1 . CeHRes Roadmap for eHealth development.

The value specification builds on the results of the contextual inquiry and here the key stakeholders determine and rank their values. These values are cooperatively translated into requirements of the technology. In this study, this was done by conduction interviews combined with rapid prototyping (Kinzie et al., 2002) with prospective users, and a requirement session with the project development team, consisting of researchers, designers and programmers.

In the design step, (a prototypical version of) the technology is developed, based on the requirements. The framework states that the quality of the design can be assessed at the levels system quality (user friendly application that matches the end-users' role and task), content quality (providing meaningful and persuasive information) and service quality (providing an adequate and feasible service that fits the context) (DeLone and McLean, 2003). In our study, this was done by conducting 
an expert-based usability inspection, a user-based usability evaluation and a requirement session with the project development team.

The goal of this study is twofold. The first goal is to create a user-friendly application which fits the values of the stakeholders and which can be implemented in daily routine. Our second goal is to evaluate the process of development. The significance of this study is obvious for the application being developed, but additionally, the results regarding the actual application and the process of development can be used as a vantage point when developing similar web-based applications.

In the following sections, of each step of the CeHRes roadmap that we undertook, the method and results will be described. We have chosen to present these sections per step, to increase clarity and to retain the iterative process of the development. The study was conducted between January and March 2010.

\section{Contextual inquiry}

\section{Methods}

To gain a better insight in the context of web-based interventions for the indicated prevention of depression, we performed an exploratory literature scan to gain an overview of the context of our to be developed web-based intervention. Specific goals of the literature scan were: gaining insight in the need for a web-based intervention for the indicated prevention of depression, in features that might enhance the effect of a web-based intervention, and in the effectiveness of the course that provides the content for the web-based intervention. Additionally, we discussed the goals of the project and the needs of project team with the project management team.

\section{Results}

The literature scan revealed a supposed need for easily accessible interventions for the indicated prevention of depression (Beekman et al., 2010; Cuijpers et al., 2008; Cuijpers et al., 2010; Hollon et al., 2002; Riper et al., 2007; van Baal et al., 2008; van Voorhees et al., 2011). Web-based guided self-help interventions are frequently mentioned as potentially meeting this need (Beekman et al., 2010; Cuijpers et al., 2010; Riper et al., 2007; van Voorhees et al., 2011). However, this need is mostly stated by care professionals and researchers, for the intended clients.

Our literature scan for features that might enhance the effect of a web-based intervention found strong indications that providing support is essential for an intervention to be effective (Andersson and Cuijpers, 2009; Mohr et al., 2011). Furthermore, there is indicative evidence for the effectiveness of using additional text messages to communicate with participants (Webb et al., 2010). This is strengthened by the 
results of a systematic review that shows that periodic prompts can enhance the effectiveness of web-based interventions (Fry and Neff, 2009). Lastly, diaries for self-monitoring have been shown to be successful in a variety of mental health conditions (Thiele et al., 2002) and have been advocated as a useful persuasive technology feature (Fogg, 2003; Oinas-Kukkonen and Harjumaa, 2009).

The content of the web-based intervention that will be developed, is based on the self-help book "Living to the Full" (Bohlmeijer and Hulsbergen, 2008). This intervention is based on acceptance and commitment therapy (ACT; Hayes et al., 1999) and mindfulness (Kabat-Zinn, 1990, 1994), and targets experiential avoidance that can be considered as a generic risk factor for mental illnesses (Biglan et al., 2008). Several studies show the effectiveness of this intervention as indicated prevention for depression for a similar target group as is intended for the web-based intervention (Bohlmeijer et al., 2011; Fledderus et al., 2012; Fledderus et al., 2010). Studies of Bohlmeijer et al. (2011) and Fledderus et al. (2010) showed that "Living to the Full" as a group course is effective in substantially reducing depressive symptomatology and improving positive mental health in adults. A second study of Fledderus et al. (2012) showed that the ACT-based intervention as a self-help program with both minimal and extensive email support was effective for people with mild to moderate depressive symptomatology.

During the discussion of the goals of our development study and the needs of the project team, we decided that the web-based intervention that will be developed, is, at this stage, primarily a research tool. The reasons for this decision were on the one hand practical: there was no specific implementation setting for which the intervention could be developed (e.g. there was no participating Mental Health Care Organization). On the other hand, this decision was made because the project team wanted to be able to experiment with the web-based intervention, which was decided to be most practical when the intervention would be developed as a research tool. In line with this decision, the most important stakeholder groups were decided on. Because of the choice for a research implementation setting, researchers were deemed an important stakeholder group and, in this stage, care providers were less prominent in the development process. Again, this decision was, in part, a practical decision. The care providers in the research setting were a member of the project team (who was involved) and students of the University who will participate in the counselling of future participants of the intervention, but were not known at the time of the study. 


\section{Value specification}

\section{Methods}

In this stage it was determined which values (in this respect, values are anything that a stakeholder deems important related to the goals of the web-based intervention and can be socio-economical as well as behavioural) the prospective users deem important and how they could be implemented in the design of the intervention. This was done by investigating the expected needs of prospective end-users, i.e. people with mild depressive symptoms who were willing to participate in a preventive intervention, using interviews combined with rapid prototyping. Needs are seen as an amplification of the often more abstract values and are expected to be easier voiced by the participants. To translate the expected needs into requirements, a requirement session with the project management team was held.

Interviews combined with rapid prototyping

Semi-structured interviews were performed to identify general expected needs of the target group and specific expected needs regarding the usefulness of the features that came forward in the contextual inquiry. The interviews were combined with rapid prototyping (Kinzie et al., 2002). In total, 18 interviews were conducted, which was a similar number of participants used in the study described by Kinzie et al. (2002). The interview participants were people that were interested in participating in a previous study into the effectiveness of "Living to the Full" as a guided self-help format with e-mail support (Fledderus et al., 2012), but could not enrol in that study because the maximum number of participants was reached. All participants received a gift voucher for their participation. Prior to the interview, the interviewer explained the goal and process of the interview, obtained permission to audio record the interview and each interviewee signed an informed consent. A typical interview lasted about 45 minutes.

The interview scheme was based upon eHealth and Human Centered Design literature (Gulliksen et al., 2003; Kinzie et al., 2002; Kukafka et al., 2003; van Gemert-Pijnen et al., 2011) and consisted of three parts. Part one focused on previous experience with the content of the intervention and with web-based interventions in general to assess the background and experience of the participants. Additionally, the expected needs were discussed by asking three cruxes that the web-based intervention had to satisfy and one aspect that would be a reason to quit (or not to start) the intervention. Rapid prototyping was part two of the interview and focused on the usefulness of three features that were available as a paper prototype, i.e. text message coaching, online diary and support in the form of a feedback message. Furthermore, satisfaction with the design and usefulness of the general application was 
assessed by asking the participants to comment on two different designs of a general home page, a personal homepage and a page with an assignment within the course. The general homepage of the first design and the personal homepage of the second design are presented in Figure 2. All paper prototypes can be found in Additional file 1. Part three assessed demographics, such as age, education and Internet experience. The interviews were analysed within 48 hours after the interview had taken place (Holtzblatt et al., 2005). We used an inductive thematic analysis to identify patterns in the responses (Braun and Clarke, 2006). All analyses were done by two independent coders ( $\mathrm{SK}$ and $\mathrm{MO}$ ).

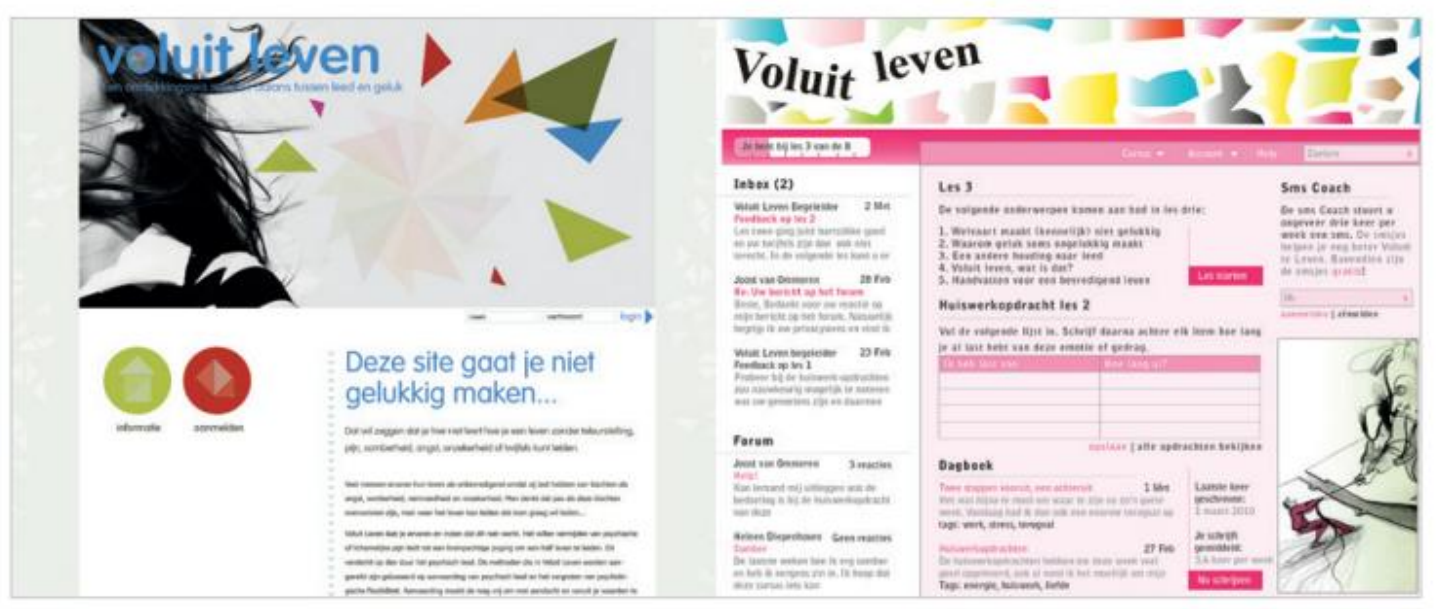

Figure 2 - Paper Prototypes: General Homepage, First Design (Left); Personal Homepage, Second Design (right).

\section{Requirement session}

The results of the interviews and rapid prototyping were communicated to the designers and programmers by means of a report written by the researchers. The report consisted of the thematic analyses of the responses of the participants. The responses were summarized and quantified where possible, but no interpretation was made by the researchers. During the discussion of the results with the project development team, the responses were translated into requirements. The researchers addressed the expected needs and categorised comments of the participants, and clarified them when they were unclear to the designers and programmers. The designers and programmers used their expertise to fit the expected need or comment in a requirement. The researchers verified whether the requirement did truly meet the expected need or comment by checking the actual comments as they were expressed by the participants. 


\section{Results}

\section{Participants}

The mean age of the 18 participants was 45 years (range $26-62, s d=10), 78 \%(n=14)$ was female and $78 \%(n=14)$ completed at least higher vocational education. Of the participants 50\% $(n=9)$ had no experience with mindfulness or meditation, $33 \%$ $(n=6)$ had experience with meditation, $6 \%(n=1)$ had experience with mindfulness and $11 \%(n=2)$ had experience with the course "Living to the Full”. Self-reported experience with using the Internet was high for $39 \%(n=7)$, medium for $56 \%(n=10)$ and low for $6 \%(n=1)$ of the participants. After the interview $78 \%(n=14)$ indicated that they would use the course when available as a web-based intervention, $6 \%$ $(n=1)$ would not use the application and $17 \%(n=3)$ were not sure whether they would use the application or not.

\section{Expected needs}

The expected needs that were mentioned by the participants were inductively categorised (Table 1; see Additional file 2 for an overview of the specific expected needs). Almost all participants $(n=17)$ expected a need for professional support and feedback. These needs were formulated as, for example, "The system needs to provide contact with a counsellor" ( $n=8)$ or "The application needs to provide feedback on your progress" $(n=8)$. A need specifically targeted at the system itself was expected by $78 \%$ ( $n$ = 14). "The application needs to be user friendly" was the most often mentioned need $(n=12)$. Content needs were expected by 13 participants $(72 \%)$, where they most often needed the content to have added value $(n=8)$ and be effective $(n=6)$. A service need was expected by $56 \%(n=10)$. Service needs related to the process of receiving care through technology, for example, "The course needs to have a flexible time planning" $(n=5)$ or "The course needs to have a fixed endpoint" $(n=3)$. Finally, 8 respondents (44\%) expected a need for contact with others using the application (peer support).

Table 1 - Categories of expected needs of prospective users.

\begin{tabular}{lll}
\hline Category & $\mathbf{n}$ & $\%$ \\
\hline Professional support and feedback & 17 & 94 \\
System & 14 & 78 \\
Content & 13 & 72 \\
Service & 10 & 56 \\
Peer support & 8 & 44 \\
\hline
\end{tabular}




\section{Usefulness of features}

Of the participants, $67 \%(n=12)$ indicated that they would use text message coaching. $11 \%(n=2)$ would not use this feature and for the remaining $22 \%(n=4)$ using this feature would depend on the content of the text messages. Reminders $(n=11)$ are seen as the most useful content for the text messages, although assignments $(n=6)$ and motivation $(n=4)$ were also seen as useful content. The possibility of an online diary was received with mixed reactions. Of the respondents, $44 \%(n=8)$ would definitely use it and saw it as a pleasant addition. However, $56 \%(n=10)$ would only use the diary when it is a part of an assignment in the course. All respondents indicated that support in the form of feedback messages would be useful and essential. Feedback is expected on assignments, but furthermore, it could be useful for gaining new insights, support and motivation. Several respondents $(n=5)$ stress the importance of the feedback being personal.

\section{Feedback on design}

For both the homepage and the exercise, the first paper prototype was preferred ( $56 \%$ and $77 \%$ respectively). Positive associations with the first prototype were "professional” ( $(n=5)$, "calm" ( $(n=5)$ and "clear" $(n=3)$. Negative associations were less common, but "bleak" was mentioned by 2 respondents. For the second prototype the most mentioned association was "busy" $(n=5)$, followed by "cheerful" $(n=4)$. Furthermore, comments of the participants on both paper prototypes regarded the appropriateness and attractiveness of the used images, shapes, header, colour, font, text, buttons, menu structure and process indicator. An example of the written comments of two participants on two paper prototypes is presented in Additional file 3.

\section{Requirement session}

The results of the interviews were discussed with the project development team. The expected needs of the participants that were not yet met in the design of the web-based intervention were discussed regarding urgency, feasibility and desirability according to the stakeholders (participants, researchers, designers and programmers). This resulted in requirements regarding the system, the service and peer support, where for example for peer support, it was chosen not to fully incorporate the need of the participants for "contact with others using the application" but to include prewritten experiences of people who used the self-help book "Living to the Full". The comments of participants regarding the appropriateness and attractiveness of the used images, shapes, header, colour, font, text, buttons, menu structure and process indicator were discussed and translated into requirements that were implemented in the prototype. Finally, a time planning for building the prototype was agreed upon, including another requirement session where the progress was discussed. 


\section{Design}

\section{Methods}

A working prototype of the web-based intervention was developed according to the requirements specified in the value specification stage. The prototype consisted of the registration procedure, the first lesson of the course and the personal home page with the features: testimonials, diary, text message coach, overview of completed assignments and overview of feedback. The prototype was evaluated on system quality, content quality and service quality by conducting an expert-based usability inspection and a user-based usability evaluation. In line with the recommendation by Jaspers (Jaspers, 2009), we have chosen to employ both a user-based and an expert-based evaluation method. We have investigated issues identified by these methods and the overlap of identified issues by both methods. This knowledge will help us make founded choices on which methods to use in similar development processes. Issues that came forward using these evaluation methods were combined into points for improvement, which were discussed with the development team to translate these points into requirements and prioritise these requirements.

\section{User-based usability evaluation}

We employed a scenario-based think aloud protocol (Jaspers, 2009), i.e. prospective users were guided through the application by means of scenarios that pose a problem or task that may be solved or completed by using the program, and respondents were instructed to verbalise their thoughts during the whole test. We conducted usability tests with ten prospective users, which is deemed sufficient to identify the main usability problems (Nielsen, 1994). Participants were recruited using online advertisements and were part of the target group of the web-based intervention. All participants received a gift voucher for their participation. Prior to the usability test each participant signed an informed consent. All usability tests were recorded and coded retrospectively. The material (audio and video) was reviewed and comments were identified by the researcher. Comments were defined as relevant verbalisations of a thought, problems encountered by the participants, tasks that were completed smoothly and relevant feedback the participant provided during the interview. These comments were analysed using a coding scheme following the work of DeLone and McLean (DeLone and McLean, 2003) and Van Gemert-Pijnen (van Gemert-Pijnen et al., 2011), which distinguishes between system quality, content quality and service quality. System quality refers to the user friendliness of the application, including the placement of buttons and the lay-out of the application. Content quality refers to the usefulness and persuasiveness of the information presented in the application, including spelling and understandability of all texts in the application. Service quality 
refers to the process of care given by the application, including the registration procedure and features that have (not) been included.

\section{Expert-based usability inspection}

The cognitive walkthrough method (Jaspers, 2009) was used to assess the usability of the application by experts. In a cognitive walkthrough, experts analyse and evaluate the steps a typical user would take when trying to reach a certain goal. Important in a cognitive walkthrough is that it is specifically guided by user tasks or goals. The experts that carried out the cognitive walkthrough were all eHealth researchers and were working at the University of Twente. One of the experts is a clinical psychologist and has expert knowledge about the target group. Issues were coded using the same coding scheme as used for user-based usability evaluation. To check for differences in coding, $20 \%$ of the results from the user-based usability evaluation and $20 \%$ of the results of the expert-based usability inspection were coded by two researchers (SK and JvG). The interrater reliability, measured by Cohen's kappa, was 0.84 for the categories "content", "system" and "service", and o.go for the categories "positive", "neutral" and "negative".

\section{Requirement session}

The report written by the researchers contained the points for improvement, which were the summarized comments from both evaluation methods. The points for improvement were clustered along different parts of the prototype. For each point, the non-interpreted issue was present, but in some cases, a recommendation was added by the researcher, based on the results of the evaluation methods. During the discussion of the results with the project development team, the points for improvement were translated into requirements. The researchers addressed the points for improvement and clarified them when they were unclear to the designers and programmers. The designers and programmers used their expertise to fit the point for improvement in a requirement. The researchers checked whether the requirement did truly meet the point for improvement as it was expressed by the participants. Finally, a prioritisation was made considering on the one hand the frequency and urgency of an issue and on the other hand the prediction of time and effort to implement the new requirement.

\section{Results}

\section{Participants}

The mean age of the 10 participants of the usability test was 38 years (range 24-53, $\mathrm{sd}=11), 90 \%(\mathrm{n}=9)$ was female and $70 \%(\mathrm{n}=7)$ completed at least higher vocational 
education. The cognitive walkthrough was carried out by 8 participants, who can be categorised as usability experts $(n=7)$ and a target group expert $(n=1)$ (Jaspers, 2009).

Evaluation of system, content and service quality

In total, both methods yielded 476 comments, virtually equally distributed between the user-based and expert-based evaluation method (respectively $52 \%(n=246)$ and $48 \%$ $(n=230))$. Table 2 shows the distribution of the comments over system, content and service quality and the amount of positive (+), neutral (+/-) and negative (-) comments.

Table 2. Number of comments yielded from user-based and expert-based methods.

\begin{tabular}{|c|c|c|c|c|c|c|c|c|c|}
\hline & \multicolumn{4}{|c|}{ Users } & \multicolumn{4}{|c|}{ Experts } & \multirow[t]{2}{*}{ Total } \\
\hline & + & $+1-$ & - & total & + & $+1-$ & - & total & \\
\hline System & 50 & 7 & 98 & 155 & 26 & 2 & 99 & 127 & 282 \\
\hline Content & 18 & 7 & 35 & 60 & 7 & 2 & 57 & 66 & 126 \\
\hline Service & 9 & 2 & 20 & 31 & 2 & 3 & 34 & 39 & 70 \\
\hline Total & 77 & 16 & 153 & 246 & 35 & 7 & 190 & 232 & 478 \\
\hline
\end{tabular}

Chi-square analyses show that there were no significant differences in the distribution of comments on system, content and service between both evaluation methods $\left(X^{2}=3.57 ; p=0.168\right)$. There was a significant difference in the distribution of positive, neutral and negative comments between both evaluation methods $\left(X^{2}=22.9 ; p<0.001\right)$, where the user-based method yielded relatively more positive comments and the expert-based method yielded relatively more negative comments.

The subject of positive comments was similar between users and experts. Positive comments on the content were mainly on the texts, which were evaluated as recognizable and easy to read, as well as on the exercises (e.g. the audio mindfulness exercise and choosing a picture that represents your motto and placing this on the homepage), which were deemed fun and useful. Positive comments on the system were mainly on the ease of use of the application (e.g. easy to find specific features, no explanation required) and on the appearance (e.g. fresh, pleasant). Positive comments on the service were mainly on features that were included as the possibility to personalise the homepage through the exercises and text message coaching. Neutral comments were infrequent and were made on, for example, the 
content of texts and the procedure of doing exercises. All negative comments were clustered on subject and transformed into points for improvement, where multiple comments on the same subject were combined in one point for improvement. The points for improvement were clustered along different parts of the prototype. An overview of points for improvements that arose from the user-based method, the expert-based method and both methods can be found in Table 3 .

Table 3 - Points for improvement from user-based evaluation method, expert-based evaluation method and both evaluation methods.

\begin{tabular}{lcccc}
\hline & Users & Experts & Both & Total \\
\hline Registration & 2 & 16 & 11 & 29 \\
Textual & 2 & 8 & 4 & 14 \\
Lesson 1 & 2 & 5 & 6 & 13 \\
Cockpit & 3 & 3 & 5 & 11 \\
Exercise 1 ("Backpack with suffering") & 1 & 2 & 5 & 8 \\
Exercise 2 ("Choose your motto and picture") & 3 & 1 & 3 & 7 \\
Diary & 2 & 5 & 0 & 7 \\
Login & 0 & 3 & 1 & 4 \\
Mydata & 2 & 1 & 1 & 4 \\
Overview of exercises & 0 & 3 & 1 & 4 \\
Exercise 3 ("Audio: Bodyscan") & 1 & 2 & 0 & 3 \\
Other & 0 & 5 & 1 & 6 \\
Total & 18 & 55 & 38 & 110 \\
\hline
\end{tabular}

Of the points for improvement, $49 \%$ came forward in both methods, $16 \%$ came forward only in the user-based method and 35\% came forward only in the expertbased method. Most points for improvement were found in the registration ( $26 \%$ ). Many of these were on the content (e.g. be consistent in answering categories, the answering categories for "most important complaint" are too limited, some questions are unclear) and layout (e.g. reduce the amount of scrolling needed, align all answering categories). Furthermore, many textual and spelling points for improvement were found (13\%). Lesson 1 and the cockpit also received a substantial number of points for improvement ( $12 \%$ and $10 \%$, respectively). In the lesson, many layout improvements were deemed necessary (e.g. the text is too small, the line distance and alignment is not consistent). Furthermore, the procedure could be improved 
(e.g. it is a lot of reading before the lesson becomes interactive, the table of contents doesn't completely reflect the contents). On the cockpit, there were improvements needed on labelling (e.g. the "cockpit" needs to be renamed or introduced, "chapter" should be "lesson") and in the system (e.g. the "back" button of the browser needs to function correctly within the application, exercise 1 remains visible when the user returns to the cockpit). The number of points for improvement on the other parts ranged from three to eight and covered issues as the unwanted display of an error message when not selecting an image for your motto (Exercise 2), a lack of instruction for the diary and "the lay-out differs between browsers" (Other).

\section{Requirement session}

The points for improvement were discussed with the project development team, consisting of researchers, designers and programmers. Requirements and suggestions to meet the points for improvement were formulated. It was decided to build the full web-based intervention based on these requirements and suggestions. Furthermore, a detailed planning was made of when which parts of the intervention would be ready and would be available for error-checking. Additionally, different points for improvement of service quality were discussed with the project management team (including a care provider and a developer of the course "Living to the Full") to reach a decision on how to implement these points for improvement. This was done, for example, for "be clearer to participants on when telephone screening is needed for the registration procedure" and "is it always necessary that an exercise is completed before a participant can go to the next page?”.

\section{Discussion}

This study was aimed at creating a user-friendly application which fits the values of the involved stakeholders and which can be implemented in daily routine, and at evaluating the process of development. Regarding the application, we can say that the holistic view, incorporating system, content and service as well as the perspectives and values of the different stakeholders provided the opportunity to investigate and develop the technology not as merely a tool, but as an essential part of the care it is intended to provide. The methods (user-based and expert-based) used in this study seem to provide valuable feedback that reaches further than only comments on colour or the lay-out of buttons; rather it encompasses the context of the intervention. An important advantage of the user-based methods proved to be that the target audience assesses the prototype from their view and context. The experts seem to be successful in assessing the prototype from the point of view 
of a participant (hence the large overlap between results from the user-based and expert-based methods), but also seemed to have a more comprehensive view based on their experience. This strengthens the recommendation from literature (e.g. Jaspers, 2009) to involve both experts and users in the development of web-based interventions.

The overall process of development was satisfactory. The combination of iterative stages provided more insights in the goals and processes of the technology we were developing than the separate stages. Each step yielded insights that build on the knowledge from earlier steps and shaped the next step. It must be noted that the methods and steps we have described are not truly separate, but can be viewed as continuous. This entails that the results of the methods used in a step, can be used as input for different steps. For example, the interviews held in the value specification step, have also provided information on the context, which would, ideally be gathered in the contextual inquiry step.

\section{Limitations}

A limitation of this study is that it did not involve all stakeholders in all stages. The users were not involved in the contextual inquiry, while the CeHRes roadmap advocates this. We did conduct a literature scan, but we have not assessed the actual need for a web-based intervention of the target audience. We have tried to overcome this limitation by addressing the needs and expectations of the target audience in the value specification phase. Furthermore, care providers have not been included as stakeholder in this study. This decision was made in the contextual inquiry where we decided to take research as the implementation setting. This choice was not ideal, because research is only an intermediate implementation setting, not a final, viable, long term setting. We have coped with this limitation by including a care provider in the project management team, but nonetheless, when developing this application further for a different implementation setting, care providers need to be involved.

Another limitation is that the target users that were involved in this study were mainly highly educated women with almost half of these ( $46 \%$ ) between the ages of 40 and 49 years old. This seems a very specific group for an indicated prevention intervention for which no specific target group is formulated and therefore the results may not be generalisable to other web-based interventions. However, the group that participated in this study is the group that is reached by many web-based interventions (e.g. Balmford et al., 2008; Eysenbach, 2005; Kelders et al., 2011) and therefore seems appropriate for this specific web-based intervention. However, when a web-based intervention is intended for other groups, such as men or young people, attention should be paid to involve these people in the development of that intervention. 
A further limitation regarding the participants in this study is that all methods have been conducted with small groups of participants and may therefore not be generalisable. However, the number of participants in all methods match the recommended numbers (see Jaspers, 2009; Kinzie et al., 2002; Nielsen, 1994). We feel that the results of this study can be of value to others who are developing a web-based intervention by taking our results as a vantage point. Especially for an intervention targeted at the same audience it is reasonable to believe that the expected needs are similar. Additionally, it may well be that the values we identified are generalisable to the target audience of other web-based interventions as the users of these interventions are most often similar (overrepresentation of highly educated females; e.g. Fledderus et al., 2012). However, our results should only be taken as a starting point and should be verified in the target audience of the intervention. This iterative process is a core concept in the CeHRes roadmap (van Gemert-Pijnen et al., 2011) and covers not only the expected needs and usefulness of certain features, but also the effectiveness of the intervention. To assess the effectiveness of the intervention, it is necessary not only to look at the effectiveness of the intervention as a whole, but also to investigate the active ingredients of the intervention (Collins et al., 2007).

We have not assessed whether developing a web-based intervention using the CeHRes roadmap is better than developing a web-based intervention in a different way. In our view, this will always remain an issue, because it seems difficult, if not impossible or undesirable, to develop two web-based interventions using different methods, but using the same ideas or content as a starting point. However, we can say that using the methods in this study, we have been able to clarify expected needs for this web-based intervention and we have been able to adapt the intervention to these needs. Additionally, in this study we have not assessed the effectiveness or impact of the intervention. Therefore, we cannot say whether the intervention is successful in reducing depressive symptoms in the target group of the intervention. Future research will need to assess whether the target users will actually use the intervention and whether it has the intended effects. To gain information on how to redesign or refine the web-based intervention to better reach these effects, it is important to employ methods that objectively measure usage, yield qualitative feedback on the satisfaction of users and assess the (clinical) effectiveness.

\section{Conclusion}

This study has shown the importance of a structured development process of a webbased intervention for the indicated prevention of depression because: 1) it allows the development team to clarify the needs that have to be met for the intervention 
to be of use to the target audience; and 2) it yields feedback on the design of the application that is broader than colour and buttons, but encompasses comments on the quality of the service that the application offers. In this study, specific examples of what the structured development process has generated are: more attention to the process and the flow of participants in the application (what do the participants exactly have to do in each lesson, when can they proceed to the next lesson, when do they get reminders etc.); prevented us from creating a complex menu structure in which the users would have lost their way as they indicated in the rapid prototyping stage; the idea that text message coaching can not only be used for reminding participants, but also act as a short assignment and as motivation. Overall, by developing the technology, not only technical aspects are developed, but the whole process, including system, content and service is (re)designed to match the values of stakeholders. 


\section{References}

Andersson, G. \& Cuijpers, P. (2009). Internet-based and other computerized psychological treatments for adult depression: A meta-analysis. Cognitive Behaviour Therapy 38, 196-205.

Andrews, G., Cuijpers, P., Craske, M. G., McEvoy, P. \& Titov, N. (2010). Computer therapy for the anxiety and depressive disorders is effective, acceptable and practical health care: A meta-analysis. PLOS ONE 5, e13196.

Andrews, G., Issakidis, C., Sanderson, K., Corry, J. \& Lapsley, H. (2004). Utilising survey data to inform public policy: Comparison of the cost-effectiveness of treatment of ten mental disorders. British Journal of Psychiatry 184, 526-533.

Balmford, J., Borland, R. \& Benda, P. (2008). Patterns of use of an automated interactive personalized coaching program for smoking cessation. Journal of Medical Internet Research 10, e54.

Barak, A., Hen, L., Boniel-Nissim, M. \& Shapira, N. (2008). A comprehensive review and a meta-analysis of the effectiveness of internet-based psychotherapeutic interventions. Journal of Technology in Human Services 26, 109-160.

Bartholomew, L. K. (2011). Planning health promotion programs: an intervetnion mapping approach. JosseyBass: San Francisco, CA.

Beekman, A. T., Smit, F., Stek, M. L., Reynolds III, C. F. \& Cuijpers, P. C. (2010). Preventing depression in high-risk groups. Current Opinion in Psychiatry 23, 8-11.

Biglan, A., Hayes, S. C. \& Pistorello, J. (2008). Acceptance and commitment: Implications for prevention science. Prevention Science 9, 139-152.

Black, A. D., Car, J., Pagliari, C., Anandan, C., Cresswell, K., Bokun, T., ... Sheikh, A. (2011). The impact of ehealth on the quality and safety of health care: A systematic overview. PLoS Medicine 8, e1000387.

Bohlmeijer, E. T., Fledderus, M., Rokx, T. A. J. J. \& Pieterse, M. E. (2011). Efficacy of an early intervention based on acceptance and commitment therapy for adults with depressive symptomatology: Evaluation in a randomized controlled trial. Behaviour Research and Therapy 49, 62-67.

Bohlmeijer, E. T. \& Hulsbergen, M. (2008). Voluit Leven. Mindfulness of de kunst van het ervaren, nu als praktisch hulpboek [in Dutch], Boom: Amsterdam.

Braun, V. \& Clarke, V. (2006). Using thematic analysis in psychology. Qualitative Research in Psychology 3, 77-101.

Christensen, H., Griffiths, K. M. \& Farrer, L. (2009). Adherence in internet interventions for anxiety and depression. Journal of Medical Internet Research 11, e13.

Christensen, H., Reynolds, J. \& Griffiths, K. M. (2011). The use of e-health applications for anxiety and depression in young people: Challenges and solutions. Early Intervention in Psychiatry 5, 58-62.

Collins, L. M., Murphy, S. A. \& Strecher, V. (2007). The Multiphase Optimization Strategy (MOST) and the Sequential Multiple Assignment Randomized Trial (SMART). New Methods for More Potent eHealth Interventions. American Journal of Preventive Medicine 32, S112-S118.

Cuijpers, P., Van Straten, A., Smit, F., Mihalopoulos, C. \& Beekman, A. (2008). Preventing the onset of depressive disorders: A meta-analytic review of psychological interventions. American Journal of Psychiatry 165, 1272-1280.

Cuijpers, P., Van Straten, A., Warmerdam, L. \& Van Rooy, M. J. (2010). Recruiting participants for interventions to prevent the onset of depressive disorders: Possibile ways to increase participation rates. BMC Health Services Research $10,181$.

DeLone, W. H. \& McLean, E. R. (2003). The DeLone and McLean model of information systems success: A ten-year update. Journal of Management Information Systems 19, 9-30.

Donkin, L., Christensen, H., Naismith, S. L., Neal, B., Hickie, I. B. \& Glozier, N. (2011). A Systematic Review of the Impact of Adherence on the Effectiveness of e-Therapies. Journal of Medical Internet Research 13, e52.

Eysenbach, G. (2001). What is e-health? Journal of Medical Internet Research 3, ezo.

Eysenbach, G. (2005). The law of attrition. Journal of Medical Internet Research 7, en1. 
Fledderus, M., Bohlmeijer, E. T., Pieterse, M. E. \& Schreurs, K. M. G. (2012). Acceptance and commitment therapy as guided self-help for psychological distress and positive mental health: A randomized controlled trial. Psychological Medicine 42, 485-495.

Fledderus, M., Bohlmeijer, E. T., Smit, F. \& Westerhof, G. J. (2010). Mental health promotion as a new goal in public mental health care: A randomized controlled trial of an intervention enhancing psychological flexibility. American Journal of Public Health 100, 2372-2378.

Fogg, B. J. (2003). Persuasive technology: using computers to change what we think and do. Morgan Kaufmann Publishers: Boston.

Fry, J. P. \& Neff, R. A. (2009). Periodic prompts and reminders in health promotion and health behavior interventions: Systematic review. Journal of Medical Internet Research 11, e16.

Glasgow, R. E., Vogt, T. M. \& Boles, S. M. (1999). Evaluating the public health impact of health promotion interventions: The RE-AIM framework. American Journal of Public Health 89, 1322-1327.

Gould, J. D. \& Lewis, C. (1985). Designing for usability: Key principles and what designers think. Communications of the $A C M$ 28, 300-311.

Griffiths, F., Lindenmeyer, A., Powell, J., Lowe, P. \& Thorogood, M. (2006). Why are health care interventions delivered over the internet? A systematic review of the published literature. Journal of medical Internet research [electronic resource] 8, e10.

Gulliksen, J., Göransson, B., Boivie, I., Blomkvist, S., Persson, J. \& Cajander, Å. (2003). Key principles for user-centred systems design. Behaviour and Information Technology 22, 397-409.

Hayes, S. C., Strosahl, K. \& Wilson, K. G. (1999). Acceptance \& Commitent Therapy: An experiential approach to behavior change. Guilford Press: New York.

Hollon, S. D., Muñoz, R. F., Barlow, D. H., Beardslee, W. R., Bell, C. C., Bernal, G., .. Sommers, D. (2002). Psychosocial intervention development for the prevention and treatment of depression: Promoting innovation and increasing access. Biological Psychiatry 52, 610-630.

Holtzblatt, K., Wendell, J. B. \& Wood, S. (2005). Rapid contextual design: a how-to guide to hey techniques for user-centered design. Morgen Kaufmann: San Francisco, CA.

Jaspers, M. W. M. (2009). A comparison of usability methods for testing interactive health technologies: Methodological aspects and empirical evidence. International Journal of Medical Informatics $78,340-353$.

Kabat-Zinn, J. (1990). Full catastrophe living: Using the wisdom of your body and mind to face stress, pain and illness. Delacorte: New York.

Kabat-Zinn, J. (1994). Wherever you go, there you are: Mindfulness meditation in everyday life. Hyperion: New York.

Kelders, S. M., Kok, R. N., Ossebaard, H. C. \& Van Gemert-Pijnen, J. E. W. C. (2012). Persuasive system design does matter: A systematic review of adherence to web-based interventions. Journal of Medical Internet Research 14, e152.

Kelders, S. M., Van Gemert-Pijnen, J. E. W. C., Werkman, A., Nijland, N. \& Seydel, E. R. (2011). Effectiveness of a web-based intervention aimed at healthy dietary and physical activity behavior: $\mathrm{A}$ randomized controlled trial about users and usage. Journal of Medical Internet Research 13, e32.

Kinzie, M. B., Cohn, W. F., Julian, M. F. \& Knaus, W. A. (2002). A user-centered model for Web site design: Needs assessment, user interface design, and rapid prototyping. Journal of the American Medical Informatics Association 9, 320-330.

Kujala, S. (2003). User involvement: A review of the benefits and challenges. Behaviour and Information Technology 22, 1-16.

Kukafka, R., Johnson, S. B., Linfante, A. \& Allegrante, J. P. (2003). Grounding a new information technology implementation framework in behavioral science: A systematic analysis of the literature on IT use. Journal of Biomedical Informatics 36, 218-227.

Mohr, D. C., Cuijpers, P. \& Lehman, K. (2011). Supportive Accountability: A Model for Providing Human Support to Enhance Adherence to eHealth Interventions. Journal of Medical Internet Research 13, e30.

Nielsen, J. (1994). Estimating the number of subjects needed for a thinking aloud test. International Journal of Human - Computer Studies 41, 385-397. 
Nijland, N. (2011). Grounding eHealth: towards a holistic framework for sustainable eHealth technologies (Doctoral Dissertation). University of Twente: Enschede, The Netherlands.

Oinas-Kukkonen, H. \& Harjumaa, M. (2009). Persuasive systems design: Key issues, process model, and system features. Communications of the Association for Information Systems 24, 485-500.

Pagliari, C. (2007). Design and evaluation in ehealth: Challenges and implications for an interdisciplinary field. Journal of Medical Internet Research 9, e15.

Postel, M. G., De Haan, H. A., Ter Huurne, E. D., Becker, E. S. \& De Jong, C. A. J. (2010). Effectiveness of a web-based intervention for problem drinkers and reasons for dropout: Randomized controlled trial. Journal of Medical Internet Research 12, e68.

Postel, M. G., De Haan, H. A., Ter Huurne, E. D., Becker, E. S. \& De Jong, C. A. J. (2011). Characteristics of problem drinkers in E-therapy versus face-to-face treatment. American Journal of Drug and Alcohol Abuse 37, 537-542.

Riper, H., Smit, F., Van der Zanden, R., Conijn, B., Kramer, J. \& Mutsaers, K. (2007). E-mental health. High tech, high touch, high trust. Programmeringstudie e-mental health in opdracht van het ministerie van VWS. Trimbos-instituut: Utrecht.

Tate, D. F., Finkelstein, E. A., Khavjou, O. \& Gustafson, A. (2009). Cost effectiveness of internet interventions: Review and recommendations. Annals of Behavioral Medicine 38, 40-45.

Thiele, C., Laireiter, A. R. \& Baumann, U. (2002). Diaries in clinical psychology and psychotherapy: A selective review. Clinical Psychology and Psychotherapy 9, 1-37.

van Baal, P. H. M., van den Berg, M., Tariq, L., Hoogenveen, R. T., Schoemaker, C. G., Schuit, A. J. \& de Wit, G. A. (2008). Economic evaluation of prevention. Modelling the cost-effectiveness of increasing alcohol taxes and of prevention of major depression. In Economische evaluatie van preventie. Modelleringstudies naar de kosteneffectiviteit van accijnsverhogingen op alcohol en van depressiepreventie. RIVM (National Institut efor Public Health and the Environment): Bilthoven, The Netherlands.

van Gemert-Pijnen, J. E., Nijland, N., van Limburg, M., Ossebaard, H. C., Kelders, S. M., Eysenbach, G. \& Seydel, E. R. (2011). A holistic framework to improve the uptake and impact of eHealth technologies. Journal of Medical Internet Research 13, en1.

van Limburg, M., van Gemert-Pijnen, J. E., Nijland, N., Ossebaard, H. C., Hendrix, R. M. \& Seydel, E. R. (2011). Why business modeling is crucial in the development of eHealth technologies. Journal of Medical Internet Research 13, e124.

Van Voorhees, B. W., Mahoney, N., Mazo, R., Barrera, A. Z., Siemer, C. P., Gladstone, T. R. G. \& Muñoz, R. F. (2011). Internet-Based Depression Prevention over the Life Course: A Call for Behavioral Vaccines. Psychiatric Clinics of North America 34, 167-183. 


\section{Additional files}

Additional file 1. All Paper Prototypes: General Homepage, Exercise and Menu Overlay, First Design; General homepage, Personal Homepage and Exercise, Second Design.

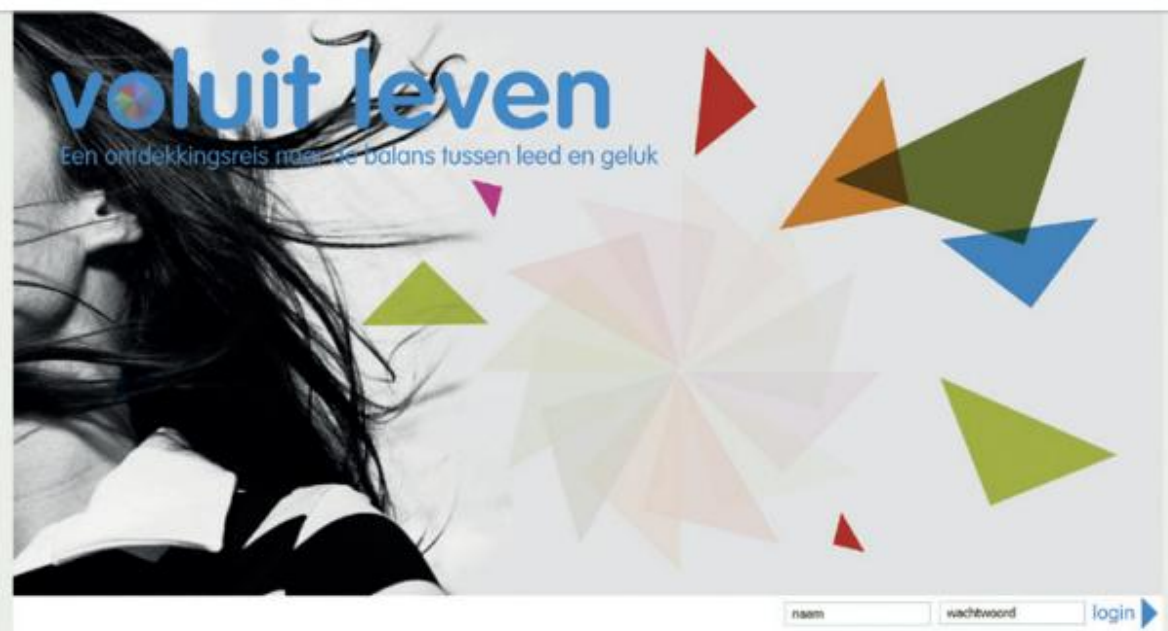

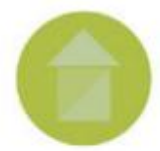

informatie

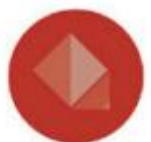

aonmelden
Deze site gaat je niet gelukkig maken...

Dat wil zeggen dat je hier niet leert hoe je een leven zonder teleurstelling. pịn, somberheid, angst, onzekerheid of twiffels kunt leiden.

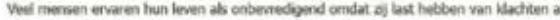

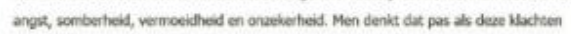

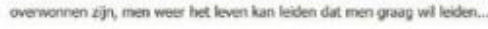

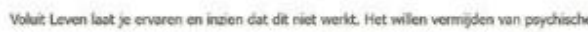

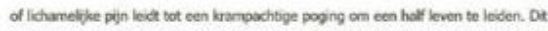

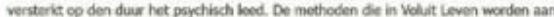

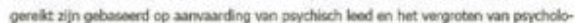

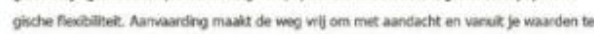

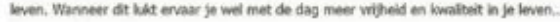




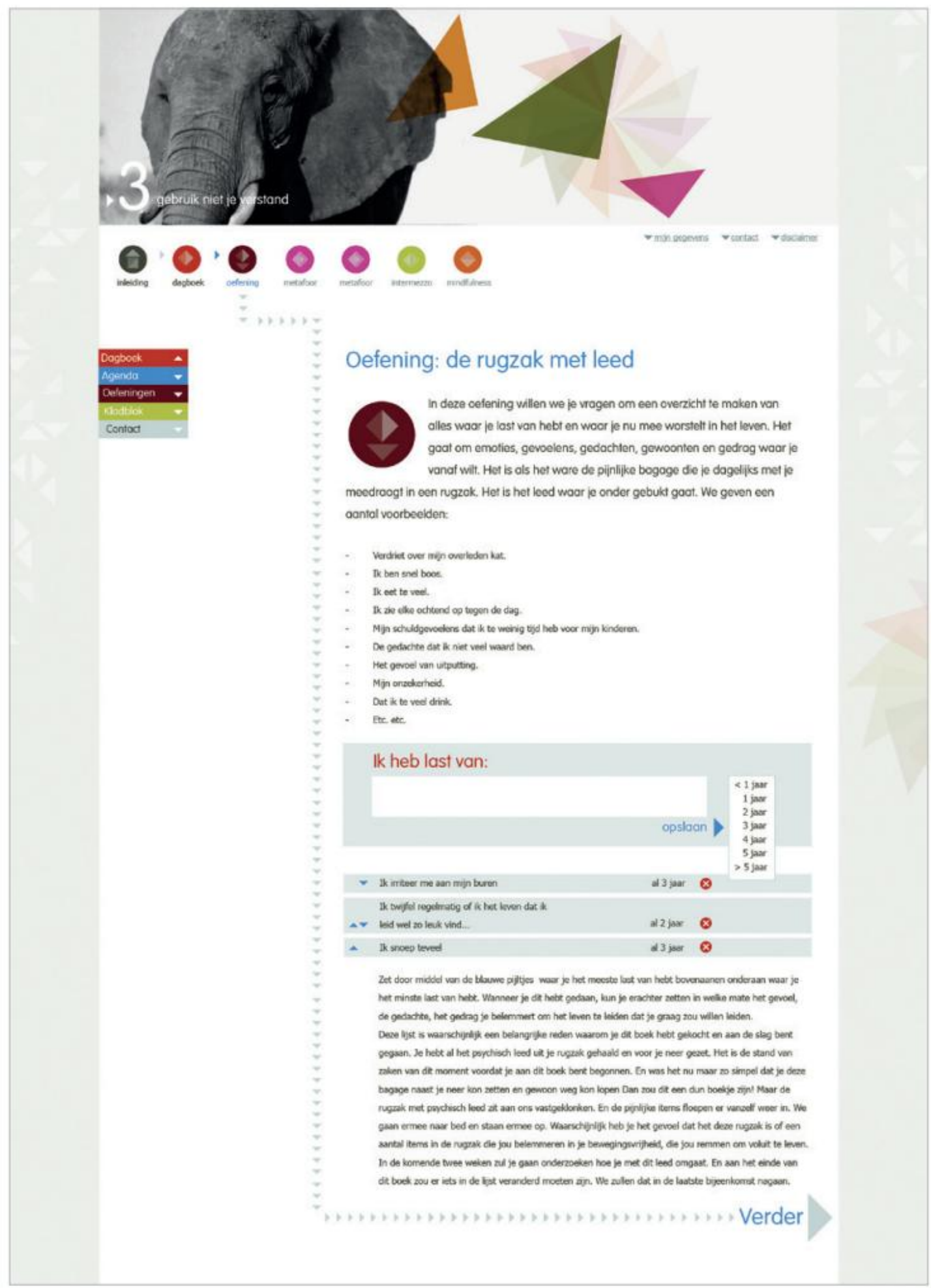




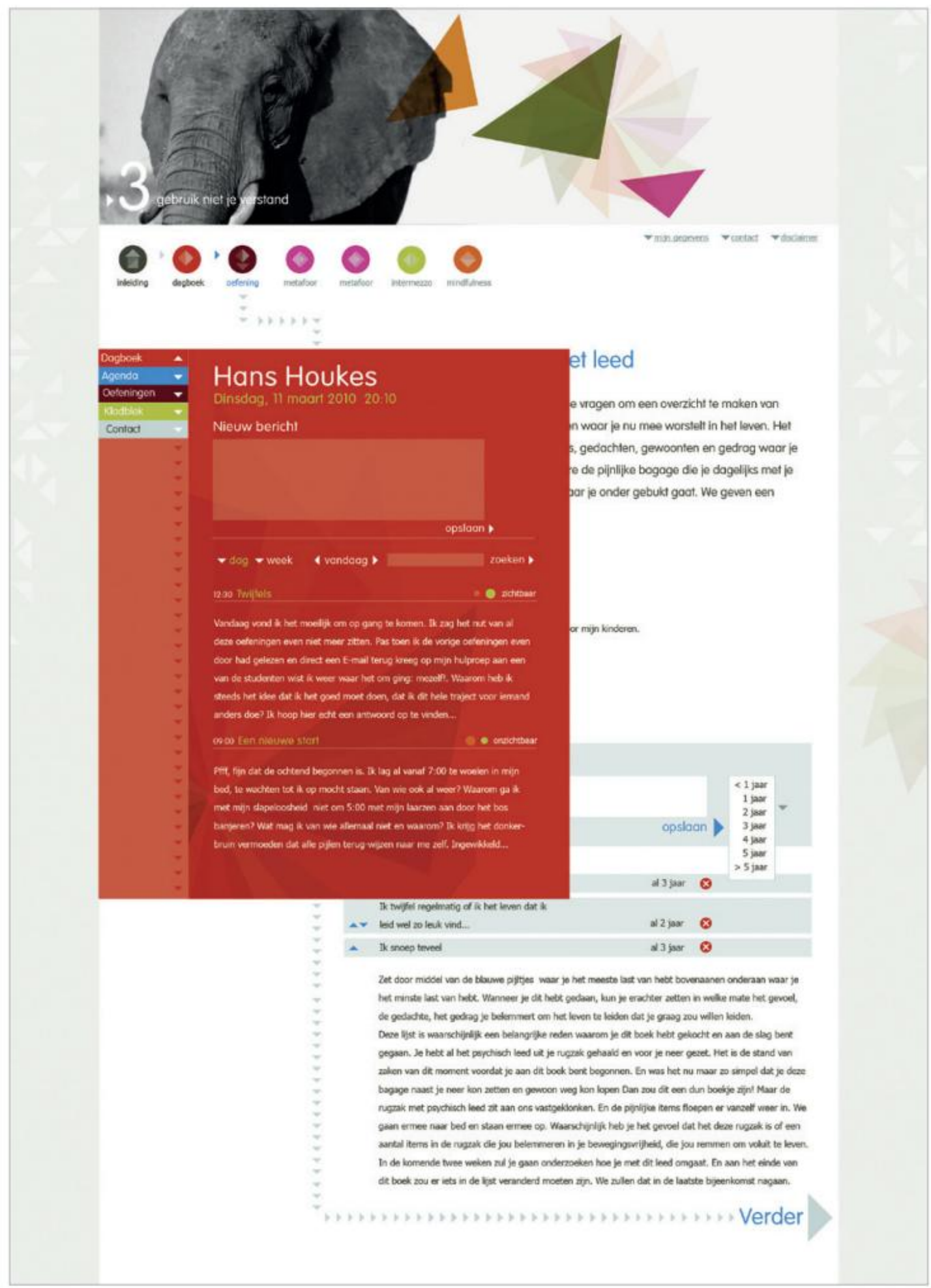




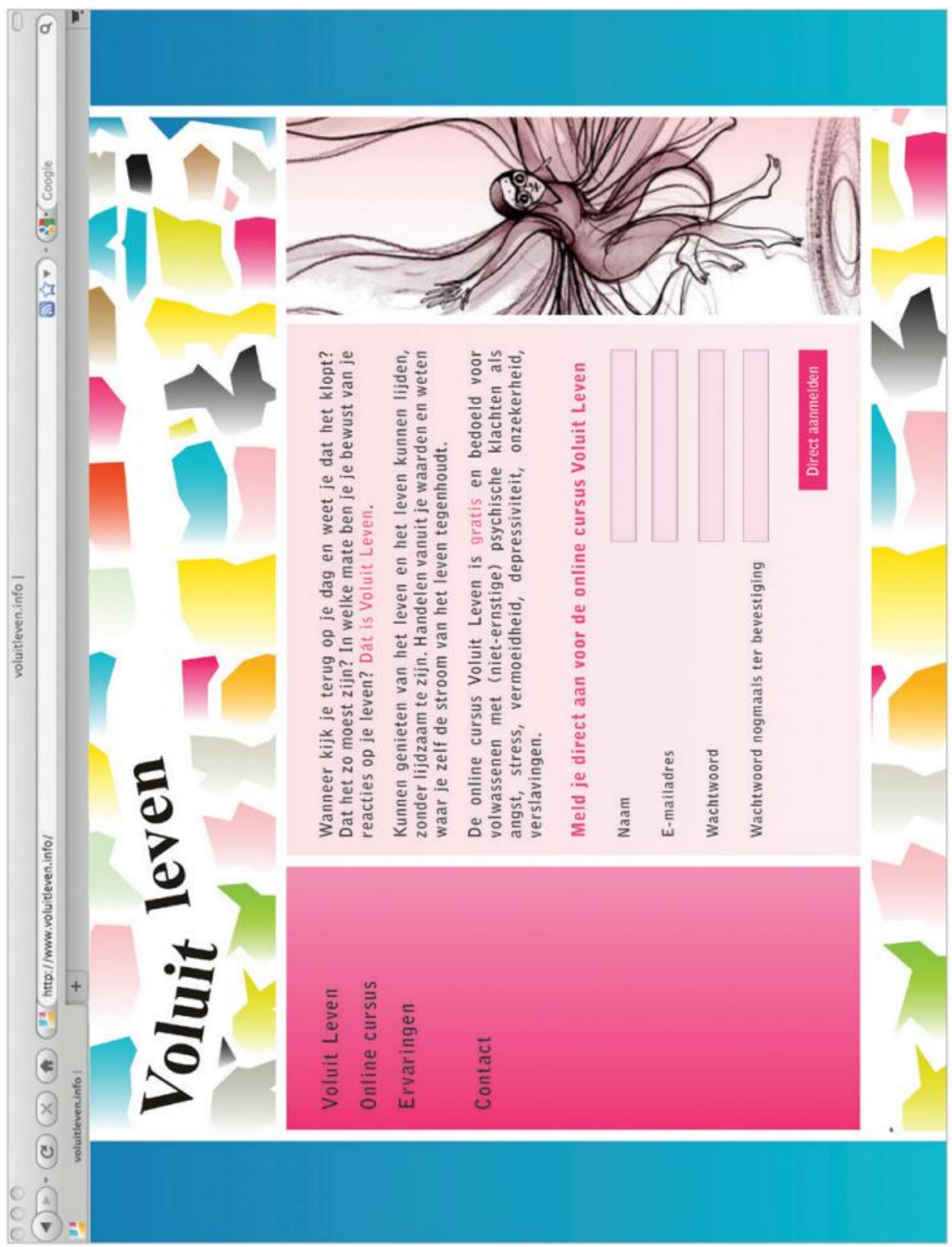




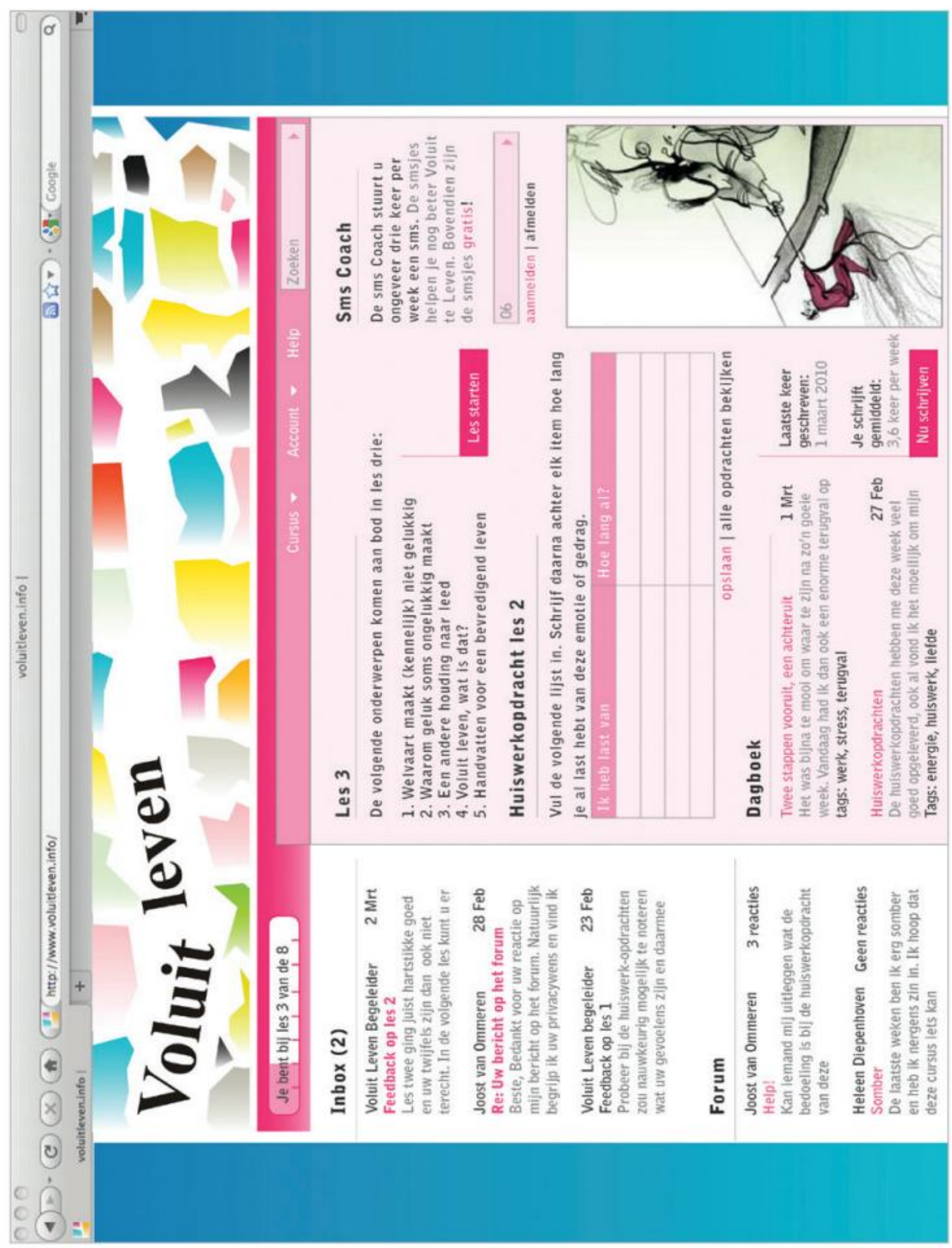




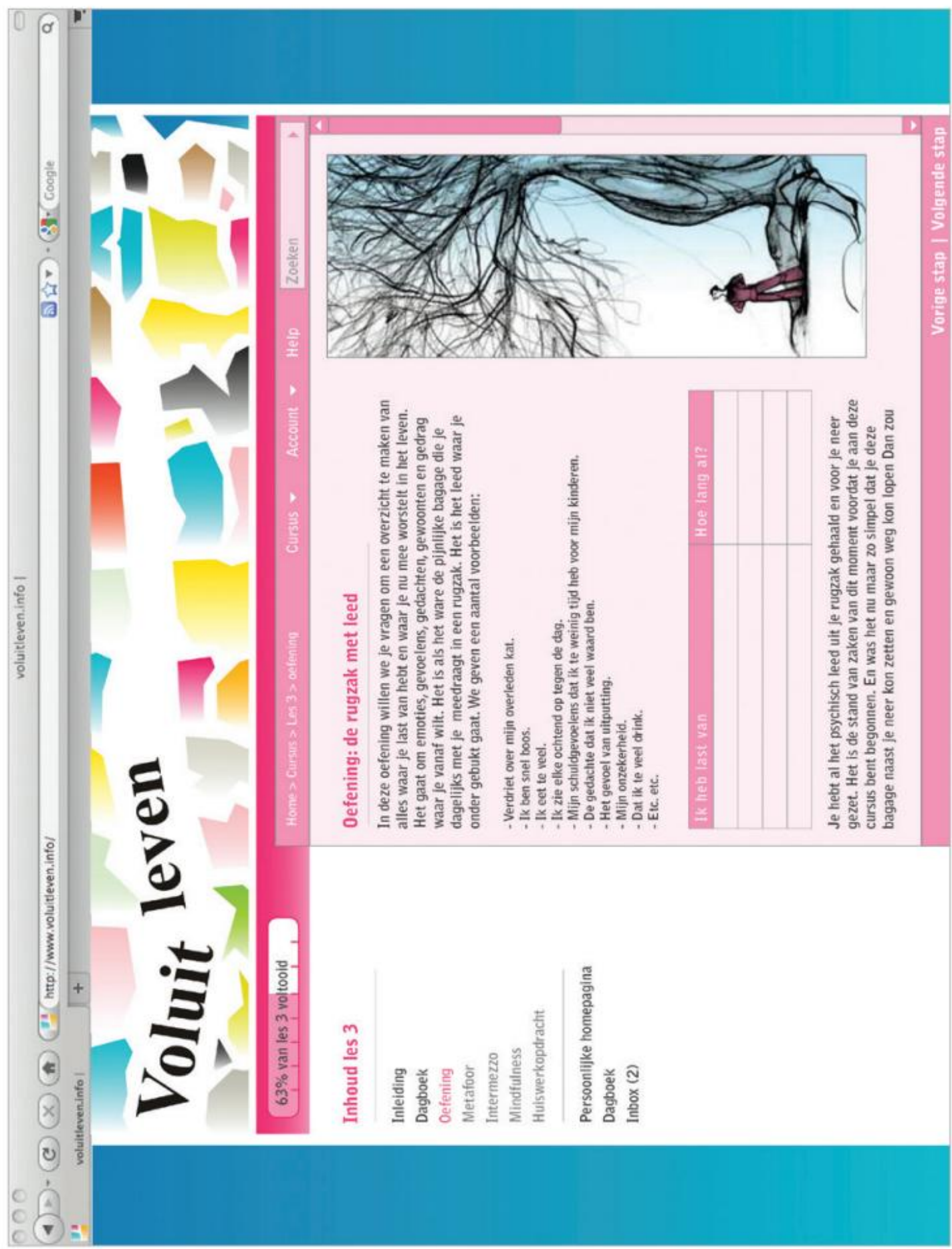


Additional file 2 - Overview of Expected Needs of Prospective Users.

\begin{tabular}{|c|c|c|}
\hline Expected Needs & $\mathbf{n}$ & $\%$ \\
\hline Support and feedback & 17 & 94 \\
\hline Contact with counsellor & 8 & 44 \\
\hline Feedback & 8 & 44 \\
\hline Personal attention & 3 & 17 \\
\hline Specific instructions & 3 & 17 \\
\hline Chat function & 1 & 6 \\
\hline Being taken seriously & 1 & 6 \\
\hline Application & 14 & 78 \\
\hline User friendly & 12 & 67 \\
\hline Attractive & 5 & 28 \\
\hline Encouragement to complete the course & 3 & 17 \\
\hline Content & 13 & 72 \\
\hline Added value & 8 & 44 \\
\hline Effective & 6 & 33 \\
\hline To-the-point & 3 & 17 \\
\hline Focus on real world & 1 & 6 \\
\hline Varied assignments & 1 & 6 \\
\hline Service & 10 & 56 \\
\hline Flexible time planning & 5 & 28 \\
\hline Fixed endpoint & 3 & 17 \\
\hline Limited time behind pc & 1 & 6 \\
\hline No need to go outdoors & 1 & 6 \\
\hline Flexible pace & 1 & 6 \\
\hline Anonymity & 1 & 6 \\
\hline Combines well with other activities & 1 & 6 \\
\hline Aftercare & 1 & 6 \\
\hline Social support & 8 & 44 \\
\hline Contact with others using the application & 8 & 44 \\
\hline
\end{tabular}


LIVING TO THE FULLEST!

Additional file 3 - Example of Written Comments of Two Participants on Two Paper Prototypes.

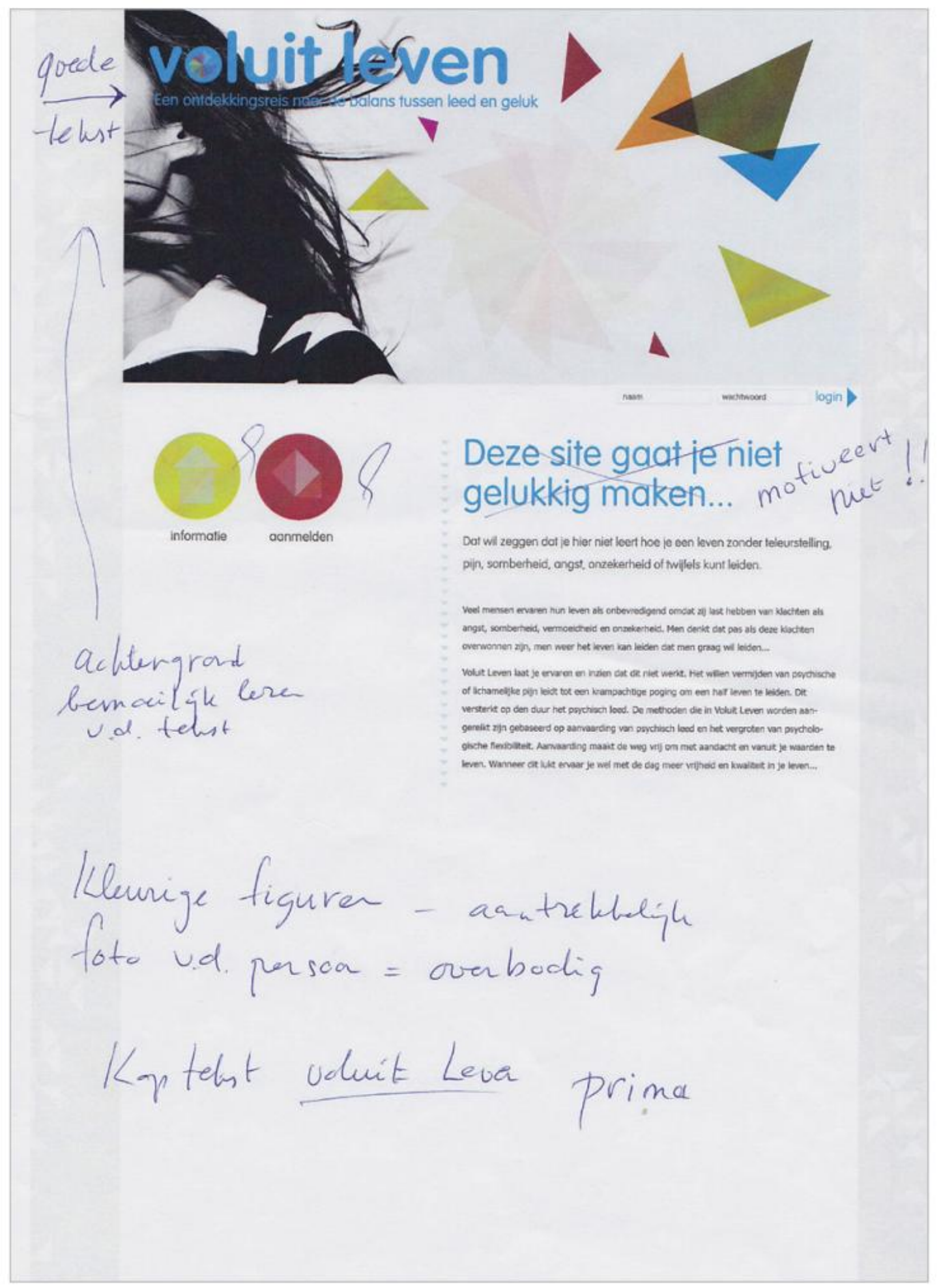

120 


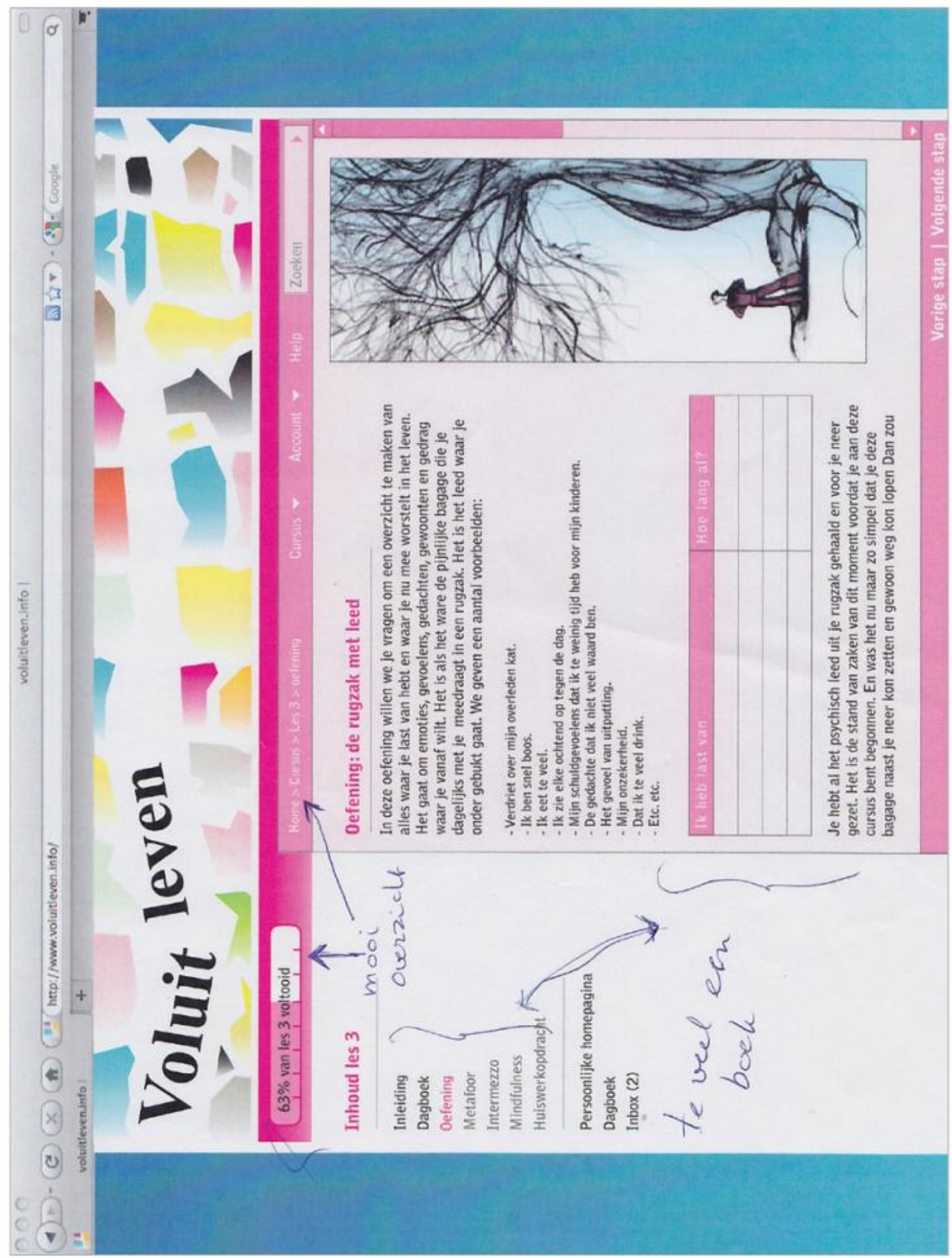




\section{CHAPTER 5}

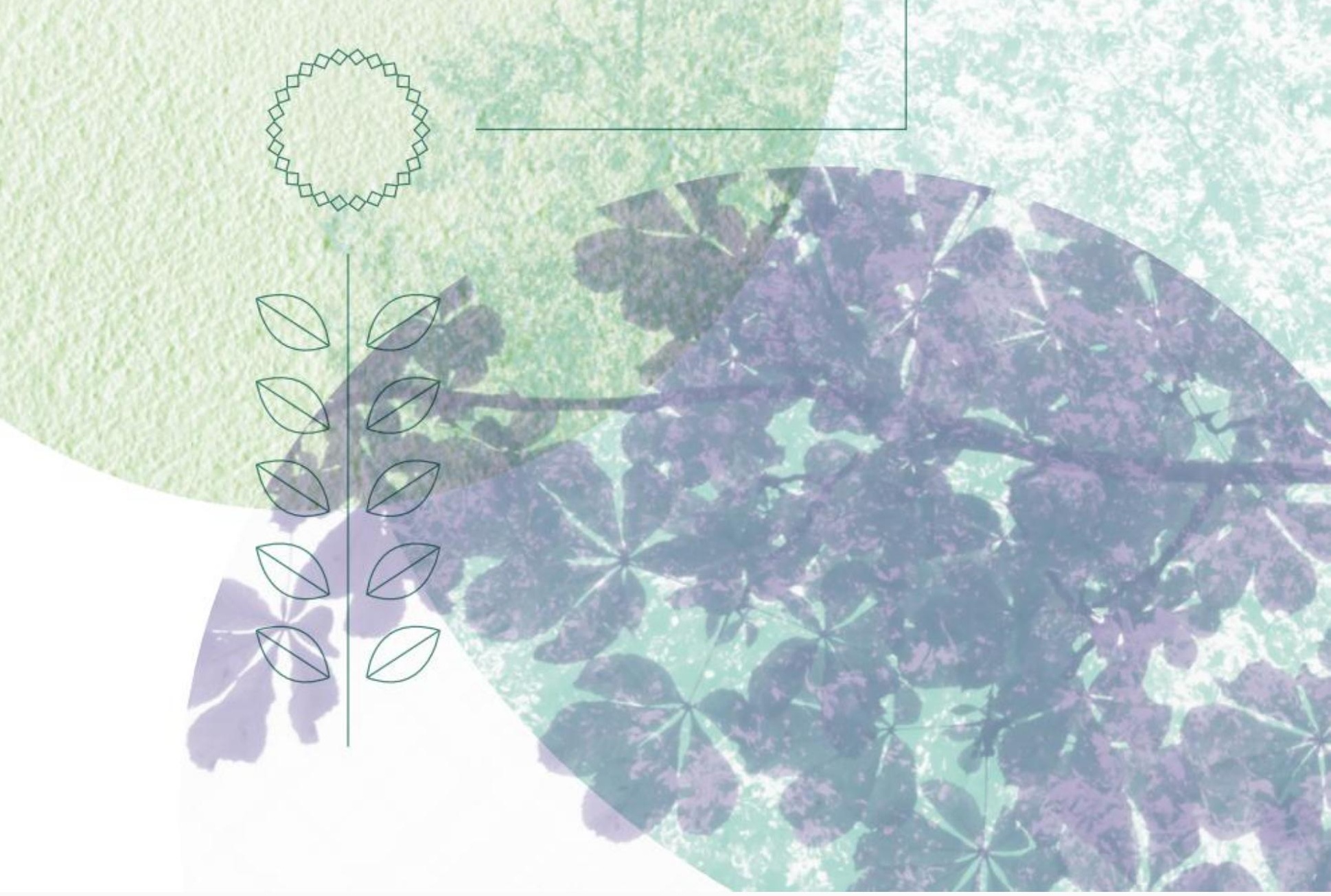

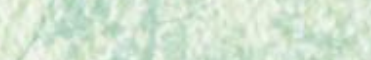




\section{Acceptance and commitment}

therapy as a web-based intervention for depressive symptoms: randomised controlled trial

Pots, W.T.M., Fledderus, M., Meulenbeek, P.A.M., ten Klooster, P.M., Schreurs, K.M.G. \& Bohlmeijer, E.T. (2015). Acceptance and commitment therapy as a webbased intervention for depressive symptoms: randomised controlled trial. British Journal of Psychiatry 208, 69-77. 


\section{Abstract}

Depression is a highly prevalent disorder, causing a large burden of disease and substantial economic costs. Web based self-help interventions seem promising in promoting mental health. The aim is to compare the efficacy of a guided web-based intervention based on acceptance and commitment therapy (ACT) with an active control (expressive writing) and a waiting-list control condition (Netherlands Trial Register NTR2736). Adults with depressive symptoms from the general population were randomised to ACT $(n=82)$, expressive writing $(n=67)$ or waiting-list control $(n=87)$. The main outcome was reduction in depressive symptoms assessed with the Center for Epidemiological Studies - Depression scale. Significant reductions in depressive symptoms were found following the ACT intervention, compared with the control group (Cohen's $d=0.56$ ) and the expressive writing intervention $(d=0.36)$. The effects were sustained at 6-month and 12-month follow-up. Acceptance and commitment therapy as a web-based public mental health intervention for adults with depressive symptoms can be effective and applicable. 


\section{Introduction}

Depression is a highly prevalent disorder with a large impact on quality of life and high economic costs (Muñoz et al., 2010). The 12-month prevalence of a depressive disorder in The Netherlands is estimated at 5.6\% for ages $18-64$ years (Bijl et al., 1998). The most important risk factor for developing major depressive disorder is the presence of clinically relevant depressive symptoms (Cuijpers and Smit, 2004). Public mental health interventions targeting people with such symptoms are a promising strategy to reduce the prevalence of depression. These interventions can be successful (Muñoz et al., 2010), but recruitment is a challenge (Cuijpers et al., 2010). Web-based interventions provide an opportunity to overcome this challenge by tackling the reasons for low participation rates, such as stigma associated with mental disorders or restrictions in time (Andersson and Cuijpers, 2008; Cuijpers et al., 2010). Meta-analyses have shown that web-based interventions based on cognitive-behavioural therapy (CBT) are effective for the prevention of full-blown depression (Arnberg et al., 2014; Richards and Richardson, 2012). One specific CBT intervention is acceptance and commitment therapy (ACT; Hayes et al., 2006). ACT is an evidence-based treatment that focuses on promoting psychological flexibility, defined as the ability to act effectively in accordance with personal values even in the presence of life adversities (Hayes et al., 2012). Despite the efficacy of ACT in various self-help formats (Cavanagh et al., 2014), its efficacy as a web-based self-help intervention for adults with depressive symptoms or mild depressive disorder has not been studied. Our aim was to examine the effects of such an intervention in a large randomised controlled trial. This is the first study to compare this therapy not only with awaiting-list control but also with an active control condition based on expressive writing (Pennebaker, 1997), and to evaluate its outcomes up to a year later.

\section{Method}

The study was approved by an independent medical ethics committee for research in mental health settings in The Netherlands (METiGG; NL33619.097.100) and recorded in The Netherlands Trial Register (NTR2736). It is a pragmatic, randomised controlled trial with three arms: the ACT intervention, an active control condition (expressive writing) and a waiting-list control condition. Based on previous results randomisation was stratified according to gender, education (low versus middlehigh) and age ( $\leq 50$ versus $>50$ years) (Bohlmeijer et al., 2011b; Fledderus et al., 2012a), using a computer-generated list that was concealed from the investigators. A sample size of 50 participants per condition was needed to detect an effect size of 
0.40 (Cohen's d) for the primary outcome (Bohlmeijer et al., 2011b; Fledderus et al., 2012a), with a statistical power of $(1-\beta)=0.80$ in a two-tailed test $(\pi<0.05)$. Taking into account a drop-out rate of $40 \%$ for web-based interventions (Christensen et al., 2009), 235 people were needed for randomisation. At 6 months after baseline those in the waiting-list control group received either the ACT intervention or the expressive writing intervention, which were offered as a choice. The assessment points were at baseline $\left(T_{0}\right)$, post-treatment 3 months after baseline $\left(T_{1}\right)$ and at 6 months $\left(T_{2}\right)$ and 12 months $\left(T_{3}\right)$ after baseline. Only participants in the intervention conditions received a follow-up measurement at $T_{3}$, since the waiting-list group received their intervention after $T_{2}$ and were excluded from later analyses.

\section{Participants and procedure}

Participants were recruited in January and February 2011 through advertisements in Dutch national newspapers and on the Internet, asking for participation in research on coping with negative emotions through the use of a free web-based intervention. A webpage created for the purpose of this study included an outline of the study design and an option to register for participation in the trial. Within the webpage candidates filled out a secured computerised informed consent form. After informed consent had been received, initial screening was conducted online for checking the inclusion and exclusion criteria by use of a self-report questionnaire in a fully automated computerised assessment battery. Individuals were invited to participate if they met the following inclusion criteria: an age of 18 years or older with mild to moderate depressive symptoms, defined as a score above 10 on the Dutch version of the Center for Epidemiological Studies - Depression scale (CESD-D; Fledderus et al., 2012a), and completion of the baseline measurement ( $\left.T_{0}\right)$. Applicants were excluded if they reported few depressive symptoms ( $\leq 10$ on the CES-D), had received psychological or psychopharmacological treatment for a mental complaint within the past 3 months, had reading or writing problems due to insufficient Dutch language skills or were unable to invest approximately $30 \mathrm{~min}$ per day up to $3 \mathrm{~h}$ per week in the intervention and daily practice. Furthermore, applicants were excluded if diagnosed with a current severe mental disorder or had a moderate to high suicide risk according to the Dutch version of the Mini International Neuropsychiatric Interview (MINI) and the Sheehan Disability Scale (SDS) (Leon et al., 1997; Sheehan et al., 1998; van Vliet and De Beurs, 2007). These individuals were excluded because they would require more intensive treatment, and were advised to consult their general practitioner. After passing the initial screening procedure all participants were contacted by telephone for a semi-structured interview using the MINI and the SDS. Together with the initial screening this constituted the baseline assessment. Five Master's degree students in psychology conducted the telephone interviews. They attended 
a 1-day workshop and were supervised during the assessments by a licensed clinical psychologist (W.T.M.P.). Decisions on participation in the study, based on the initial screening and the interview, were provided to the candidates at the end of the clinical interview with further information on participation or, when excluded, information on how to obtain adequate care. All approved participants received an email with instructions on how and when to log into the system.

All clinical interviewers were masked to the randomised condition. Participants were randomised after the clinical interview, and at $\mathrm{T}_{2}$ the contact information needed for the clinical interview was held separately from the results of the randomisation procedure. Additionally, all interviewers were instructed to state explicitly at the start of the interview that they were unaware of which condition the participants were randomised to and that this masking was needed to ensure the interview was conducted in a non-biased manner.

\section{Interventions}

\section{Acceptance and commitment therapy}

The web-based ACT intervention was based on a self-help intervention, "Living to the Full" (Bohlmeijer and Hulsbergen, 2013). This intervention has shown to be effective in promoting psychological flexibility, both as a group course and as a self-help intervention with email support (Bohlmeijer et al., 2011b; Fledderus et al., 2012a). People who are psychologically flexible also score highly on acceptance, which is seen as a more effective strategy for regulating negative emotions and thoughts than experiential avoidance, i.e. persistent and generally fruitless attempts to avoid unwanted private experiences such as feelings, thoughts and bodily sensations (Aldao and Nolen-Hoeksema, 2010; Kashdan and Rottenberg, 2010). The web-based intervention comprised nine online modules, divided into three parts. The modules were based on six core processes of ACT that together promote psychological flexibility. These core processes are acceptance (active and aware embracing of aversive internal experiences); cognitive defusion (creating a context in which undesirable functions of thoughts disappear); contact with the present moment (mindfulness); self as context (the sense of oneself as the observer of one's thoughts, feelings and experiences); values (choosing values in different life domains); and committed action (commitment to choices on the basis of these values) (Hayes et al., 2012). In the first part of the intervention participants reflect on their avoidance and control strategies and whether these are effective in the long run. In the second part participants learn how to stay in contact with their present experiences without trying to avoid or control them. Cognitive defusion and experiencing self as context are practised. In the third part the focus is on becoming aware of one's most important personal values and 
making decisions based on these values. An additional focus is relapse prevention, which includes self-management and action plans. Each module uses experiential exercises and metaphors to illustrate the ACT process, as well as text messages, tailored stories for motivation and an option to personalise the homepage. Furthermore, participants were encouraged to practise daily mindfulness exercises, designed to reduce stress (Kabat-Zinn, 1990). These exercises lasted on average 10-15 min and were provided on audio, downloadable within the web-based intervention. For a comprehensive description of the development of the intervention, see Kelders et al. (2013).

\section{Active control condition}

The active control condition was a web-based intervention based on Pennebaker's expressive writing paradigm (Pennebaker, 1997), which has shown small effects on various mental health outcomes (Frattaroli, 2006). Expressive writing generally involves asking participants to write about a highly stressful experience (particularly their deepest thoughts and feelings), usually in three or four sessions. We extended and adapted Pennebaker's method into a web-based format equivalent to that of the ACT intervention, comprising nine online sessions presented in three parts. Each session started with a psycho educational paragraph on emotions and emotion regulation, followed by instructions in the expressive writing method. This consisted of writing about emotional experiences for 15-30 min on at least 3 days a week. The first three sessions focused on expressive writing about negative experiences. In sessions four to six participants looked back at their experiences with expressive writing in the first part, and focused on emotion regulation and reappraisal of emotions; these modules were based on Gross's process model of emotion regulation (Gross and Thompson, 2007), and were added to extend the intervention to 9 weeks. In the last three modules of the intervention participants focused on writing about positive experiences and self-management for preventive purposes.

\section{Waiting-list control}

Participants in the waiting-list control group were offered no intervention but were free to access other forms of care (as were all participants once they were eligible). They were instructed that, should they encounter symptomatic deterioration or other difficulties over the course of the study, they were to seek help from their family, general practitioner or other sources, as they normally would. Six months after baseline these participants could start a web-based intervention of their choice. Based on the preliminary results of this study at $T_{2}$, the participants were advised to start with ACT. 


\section{Counselling}

Participants in the web-based ACT and expressive writing interventions were instructed to complete one session per week, and had 12 weeks in total to complete the nine sessions. After completing a session, participants wrote an email to their counsellor reflecting on the process and using the opportunity to ask questions. They could proceed to the next session after receiving personal feedback from their counsellor. The feedback messages of the counsellors contained the key learning points and goals of the completed session, feedback on the key exercises and a preview of the following session. The feedback was accompanied by positive and encouraging support. Participants received automatic email messages when they completed a session, when personal feedback was received, and to remind them to finish a session or to start a new session. A session was fulfilled when all exercises were completed.

Five psychology Master's degree students provided the email support in both the experimental and the active control conditions. They attended a 2-day workshop from licensed doctors of clinical psychology with ample experience in CBT, ACT and expressive writing (K.M.G.S. and W.T.M.P.), in which they studied the web-based interventions and practised writing emails in the roles of both client and counsellor. Each counsellor provided personal feedback to 25-30 participants during the intervention, supervised by a clinical psychologist. The counsellors were given a total of sh on the counselling of an individual participant.

\section{Assessment}

The primary outcome measure was depressive symptoms measured by the Dutch version of the CES-D (20 items, total score o-60). Higher scores mean more depressive symptoms (Haringsma et al., 2004; Radloff, 1977). Secondary outcome measures were diagnostic classification, anxiety symptoms, positive mental health, psychological flexibility and mindfulness. The diagnostic classification was assessed with the MINI (Sheehan et al., 1998; van Vliet and de Beurs, 2007), supplemented with the SDS to measure the severity of the disorder (Leon et al., 1997). Severity was defined as at least two areas of functioning with severe role impairment due to the disorder according to the SDS. The MINI and SDS assessments were conducted by telephone at $\mathrm{T}_{0}$ and $\mathrm{T}_{2}$. Anxiety was measured with the Hospital Anxiety and Depression Scale - Anxiety subscale (HADS-A; 7 items, total score o-21). Higher scores mean more anxiety symptoms (Spinhoven et al., 1997; Zigmond and Snaith, 1983). Positive mental health was measured with the Mental Health Continuum - Short Form (MHC-SF; 14 items each scored o- 5), which measures three dimensions of positive mental health: emotional, social and psychological well-being (Lamers et al., 2011). In this study the total MHC-SF score was used, with higher scores indicating greater emotional, social and psychological well-being (Keyes et al., 2008; Lamers et al., 
2011). The Acceptance and Action Questionnaire-II (AAQ-II; 10 items, total score $10-$ 70) was used to measure the participants' willingness to be in contact with negative private events, their acceptance of these events and the ability to live according to their values. Higher scores indicate greater psychological flexibility (Bond et al., 2011; Fledderus et al., 2012b). The Five Facet Mindfulness Questionnaire - Short Form (FFMQ-SF; 24 items, total score 24-120) was used to measure mindfulness in five dimensions: observing, describing, acting with awareness, non-judging and non-reactivity. Facet scores range from 5 to 25 (except for observing, which ranges from 4 to 20), with higher scores indicating greater mindfulness (Baer et al., 2006; Bohlmeijer et al., 2011a).

\section{Statistical analysis}

The Consolidated Standards of Reporting Trials (CONSORT) guidelines for randomised trials were followed (Moher et al., 2001), and the analyses were done using SPSS version 20 for Windows. Intention-to-treat (ITT) analyses were performed using the SPSS Missing Value Analysis to impute all missing data on the continuous measures with the expectation-maximisation method. This method estimates the unmeasured data based on maximum likelihood estimates using observed data on all continuous outcome measures in an iterative process (Dempster et al., 1977). To provide a comprehensive picture of the effects of the intervention, the outcomes were analysed based on ITT as well as for those completing treatment only. The completers group was defined as participants who completed at least the first six sessions, as these sessions of the ACT intervention dealt with each of the six ACT processes. One-way analysis of variance (ANOVA) and chi-squared tests were conducted to examine baseline differences between the ACT intervention and the two control conditions. To examine the differences between the conditions on all the outcome measures, a 3 (group) $\times 3$ (time) repeated measures ANOVA was used. In the case of significant timegroup interactions, post hoc analysis of covariance (ANCOVA) was used on the change scores of the outcome measures with baseline scores as covariates. To examine the change from $T_{2}$ to $T_{3}$ in the two intervention groups, a 2 (group) $\times 2$ (time) repeated measures ANOVA and paired t-tests were used. Differences in non-study treatment (participants receiving other forms of care during the trial) were analysed using chi-squared tests on the proportions of non-study treatment between the three conditions.

Effect sizes at post-intervention were calculated with Cohen's $d$ using the means and the standard deviations of the measurements of the conditions. Cohen's d is calculated as 


$$
\frac{(\text { mean } 1-\text { mean 2) }}{\mathrm{sd}_{\text {pooled }}}
$$

where

$$
s d_{\text {pooled }}=\sqrt{\left(\frac{\left(s d_{1}^{2}+s d_{2}^{2}\right)}{2}\right)}
$$

To interpret Cohen's d, effect sizes up to 0.49 were considered small, $0.50-0.79$ moderate, $0.80-1.29$ large and above 1.30 very large (Cohen, 1992). For the changes within the groups $\left(T_{2}\right.$ versus $\left.T_{3}\right)$ Cohen's $d$ was corrected for dependence among means by using the correlation between the two means (Morris and DeShon, 2002). Comparisons were two-tailed and interpreted with a significance of $\mathrm{p}<0.05$.

Using the Jacobson \& Truax method we determined the proportion of participants who made a clinically significant change on the CES-D from baseline to post-treatment (Jacobson and Truax, 1991). First, the reliable change was calculated with the reliable change index, calculated as

$$
\left(x_{2}-x_{1}\right) / S_{\text {diff }}
$$

where $x_{1}$ and $x_{2}$ are the individual's post-test and pre-test scores and $S_{\text {diff }}$ is the standard error of difference between the two test scores; $S_{\text {diff }}$ can be computed directly from the standard error of measurement $S_{E}$ as $\sqrt{ }\left(2\left(S_{E}\right)^{2}\right)$, where $S_{E}=s d \sqrt{ }\left(1-r_{x x}\right)$ and $r_{x x}$ is the test-retest reliability of the measure. Second, the recovery criterion was defined as a post-treatment score below the cut-off value of 16 for clinically relevant depressive symptoms. The score of 16 has been established in previous research as the cut-off indicating the presence of clinically relevant depressive symptoms (Beekman et al., 1997; Smit et al., 2006). A clinically significant change on the CES-D is thus defined as a reliable change between the measurements and a post-treatment score below 16. Participants who had a clinically relevant change were coded 1 (implying favourable treatment response, 'success') or o ('failure'). The binary outcome was used to calculate the odds ratio (OR) using logistic regression. Based on the clinically significant change proportions, the number needed to treat (NNT) was calculated (Cook and Sackett, 1995).

In addition to the clinically significant change, the effect on diagnosis assessed with the MINI and the SDS was analysed using logistic regression. We calculated favourable treatment response when a participant was free from depression or did not develop a diagnosed depression at $\mathrm{T}_{2}$ ('success'). This yields a binary outcome with failure coded as o and success as 1 . This binary outcome was then used to obtain odds ratios and the NNT. Differences between the proportions of change within the diagnosis group were analysed using chi-squared tests for comparisons between the two intervention conditions and the waiting-list control. 


\section{Results}

A total of 436 persons were assessed for eligibility, of whom 93 were excluded based on initial screening criteria (Figure 1). Owing to a computer error, data concerning 43 of those excluded were not stored. Reasons for exclusion based on data for the other 50 persons were insufficient time to participate $(34 \%, n=17)$, few depression symptoms $(28 \%, n=14)$, current psychological or medication treatment $(20 \%, n=10)$, problems with the Dutch language $(16 \%, n=8)$, and age less than 18 years $(2 \%, n=1)$. A total of 343 participants met the initial screening criteria for inclusion and were contacted by telephone for a structured interview. On the basis of this interview 58 participants were excluded because of the seriousness of their disorder $(n=24)$, a high suicide risk $(n=23)$, current psychopharmacological treatment $(n=1)$ or refusal to participate $(n=10)$. A further 29 respondents could not be reached by telephone at the agreed time. Of the 256 included participants, 20 did not complete the baseline measurement. Hence a total of 236 participants were randomised.

After randomisation two participants (one in each intervention group) did not start the intervention for unknown reasons. Twelve participants in the ACT group did not complete the intervention (attended fewer than six sessions), compared with 17 in the expressive writing group. The main reasons given for non-completion were personal problems and that the intervention was too time-consuming. Adherence was $84 \%$ for the ACT group, compared with $76 \%$ for the expressive writing group. In the ACT group $73 \%$ of the participants completed all nine sessions, compared with $63 \%$ in the expressive writing group; the difference was not significant $\left(X^{2}(1, n=149)\right.$ $=1.87, \mathrm{p}>0.05)$. Analyses of non-study treatment revealed no significant difference between the conditions: at $\left.T_{1} X^{2}(2, n=175)=1.36, p>0.05\right]$; at $T_{2} X^{2}(2, n=202)=2.01$, $p>0.05$. Treatment-completer analyses revealed similar results between completer and non-completer groups with regard to demographic variables and outcome measures. Also, per protocol analysis revealed similar outcomes. Therefore, only the results for the total sample on the imputed data are reported (with exception of the logistic regression of the diagnostic classification).

At $T_{1}$ (post-treatment for the intervention conditions) data were available for 199 participants (drop-out rates: ACT 13\%, expressive writing 25\%, waiting list $10 \%$ ) and at $\mathrm{T}_{2}$ (6-month follow-up for the intervention conditions and pre-intervention for the waiting-list condition) data were available for 205 participants (drop-out rates: ACT $11 \%$, expressive writing $21 \%$, waiting list $9 \%$ ). At $\mathrm{T}_{3}$ (12-month follow-up for the intervention conditions) data were available for 118 of the 149 participants (drop-out rates: $\mathrm{ACT} 13 \%$, expressive writing $30 \%$ ). 


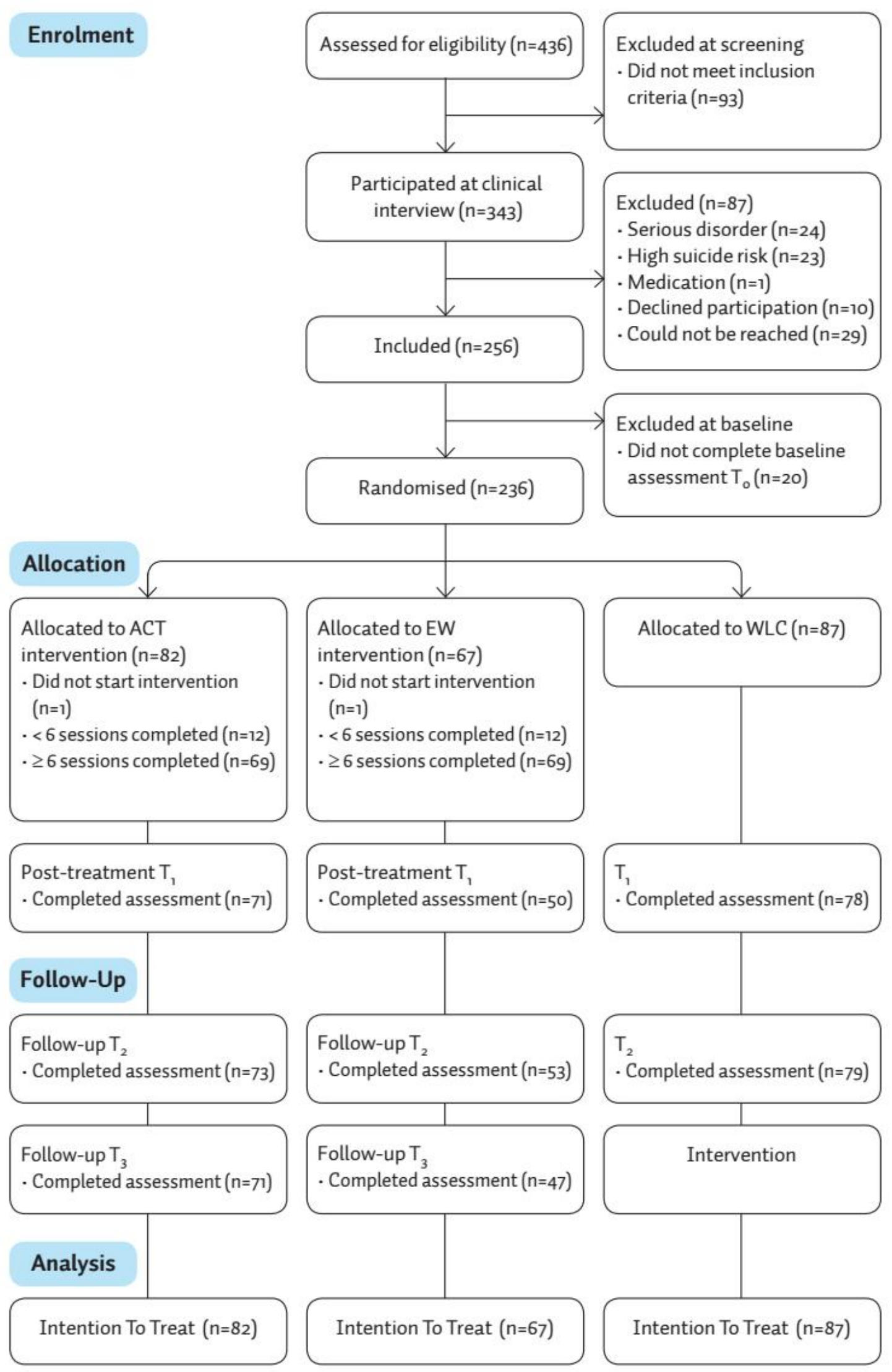

Figure 1 - Study profile. 


\section{Baseline characteristics}

Table 1 gives an overview of the participants' characteristics. Eligible participants had a mean age of 46.8 years ( $\mathrm{sd}=12.1$, range $20-73$ ), most were women $(76 \%)$, and two-thirds (66\%) had a high level of education. Mean baseline score on the CES-D was 26.73 ( $\mathrm{sd}=8.38$ ). Of the 236 participants, $95(40 \%)$ were diagnosed with a mood disorder. Owing to a programming error in the randomisation procedure the number of participants in each condition differed. There was no significant difference at baseline between the conditions for any of the demographic variables or outcome measures, indicating a successful randomisation, except for gender: $X^{2}(2, n=236)=$ $22.78, p<0.00$. A comparison of the results based on the analyses with versus without gender as a covariate revealed similar results. Therefore, only the results without gender as covariate are reported (additional analyses with gender as covariate are available from the authors on request).

\section{Outcomes}

Means and standard deviations for all outcome measures at baseline, post-treatment and follow-up and the results of the repeated measures are presented in Table 2. Figure 2 shows the repeated measure ANOVA for the primary outcome measure, the CES-D score. For all outcome measures significant interactions were found, except for the mindfulness facets observing $\left(F_{(2,233)}=2.38, p=0.05\right)$ and acting with awareness $\left(F_{(2,233)}=1.16, p=0.33\right)$. Post hoc ANCOVA revealed that participants in the ACT intervention improved significantly more from $T_{0}$ to $T_{1}$ on all outcome measures compared with the waiting-list control group (all $p<0.01$ ), except for the mindfulness facet acting with awareness $(p=0.62)$. Compared with the expressive writing intervention post hoc ANCOVA revealed that participants in the ACT group improved significantly more from $T_{0}$ to $T_{1}$ on all outcome measures (all $p<0.05$ ), except for the mindfulness facets observing $(p=0.85)$, describing $(p=0.12)$ and acting with awareness $(p=0.63)$. No significant improvement was found from $T_{1}$ to $T_{2}$ for any of the conditions on any outcome measure.

Additional analyses from $T_{0}$ to $T_{2}$ showed that the ACT intervention group improved significantly more on all outcome measures compared with the waiting-list condition (all $p<0.05)$ except for positive mental health $(p=0.06)$ and the mindfulness facets observing $(p=0.34)$ and acting with awareness $(p=0.06)$. This indicates that the ACT intervention shows a significant improvement over time compared with the waiting-list condition. The expressive writing intervention group only showed a significant improvement from baseline to follow-up compared with the waitinglist condition for psychological flexibility and the mindfulness facets describing, non-judging of inner experience and non-reactivity to inner experience (all $p<0.05$ ). 
Table 1 Baseline characteristics of the participants.

\begin{tabular}{|c|c|c|c|c|}
\hline & $\begin{array}{l}\text { Total } \\
(n=236)\end{array}$ & $\begin{array}{l}\text { ACT } \\
(n=82)\end{array}$ & $\begin{array}{l}E W \\
(n=67)\end{array}$ & $\begin{array}{l}\text { WLC } \\
(n=87)\end{array}$ \\
\hline Age, years: mean $(s d)^{a}$ & $46.85(12.06)$ & $45.15(10.78)$ & $46.73(12.65)$ & $48.54(12.63)$ \\
\hline Range & $20-73$ & $21-69$ & $20-69$ & $21-73$ \\
\hline \multicolumn{5}{|l|}{ Gender, $n(\%)^{b}$} \\
\hline Female & $179(75.8)$ & $76(92.7)$ & $40(59.7)$ & $63(72.4)$ \\
\hline Male & $57(24.2)$ & $6(7.3)$ & $27(40.3)$ & $24(27.6)$ \\
\hline \multicolumn{5}{|l|}{ Marital status, $n(\%)^{a, c}$} \\
\hline Single & $81(35.5)$ & $28(36.4)$ & $27(32.8)$ & $32(36.8)$ \\
\hline Living with partner & $147(64.5)$ & $49(63.6)$ & $43(67.2)$ & $55(63.2)$ \\
\hline \multicolumn{5}{|l|}{ Nationality, $n(\%)^{a, c}$} \\
\hline Dutch & $204(89.5)$ & $68(88.3)$ & $59(92.2)$ & $77(88.5)$ \\
\hline Other & $24(10.5)$ & $9(11.7)$ & $5(7.8)$ & $10(11.5)$ \\
\hline \multicolumn{5}{|l|}{ Education, $n(\%)$} \\
\hline High $^{a}$ & $157(66.5)$ & $55(67.1)$ & $45(67.2)$ & $57(65.5)$ \\
\hline Middle $^{a}$ & $75(31.8)$ & $24(29.3)$ & $22(32.8)$ & $29(33.3)$ \\
\hline Low & $4(1.7)$ & $3(3.7)$ & $\circ(0.0)$ & $1(1.1)$ \\
\hline \multicolumn{5}{|l|}{ Daily activities, $n(\%)^{a, c}$} \\
\hline Paid job & $150(65.8)$ & $57(74.0)$ & $41(64.1)$ & $52(59.8)$ \\
\hline No job & $78(34.2)$ & $20(26.0)$ & $23(35.9)$ & $35(40.2)$ \\
\hline \multicolumn{5}{|l|}{ Primary diagnosis, $n(\%)$} \\
\hline No diagnosis ${ }^{a}$ & $97(41.1)$ & $36(43.9)$ & $22(32.8)$ & $39(44.8)$ \\
\hline Major Depressive episode & $18(7.6)$ & $7(8.6)$ & $6(9.0)$ & $5(5.8)$ \\
\hline Recurrent depression ${ }^{a}$ & $67(25.9)$ & $21(25.6)$ & $21(31.3)$ & $19(21.8)$ \\
\hline Dysthymic disorder & $5(2.1)$ & $\circ(0.0)$ & $3(4.5)$ & $2(2.3)$ \\
\hline Other mood disorders & $11(4.7)$ & $2(2.4)$ & $4(6.0)$ & $5(5.8)$ \\
\hline Anxiety disorder ${ }^{a}$ & $44(18.6)$ & $16(19.5)$ & $11(16.4)$ & $17(19.5)$ \\
\hline \multicolumn{5}{|l|}{ Comorbidity, $n(\%)$} \\
\hline Mood disorder ${ }^{\mathrm{a}}$ & $54(22.9)$ & $22(26.8)$ & $17(25.4)$ & $15(17.2)$ \\
\hline Anxiety disorders & $3(1.3)$ & $\circ(0.0)$ & $3(4.5)$ & $\circ(0.0)$ \\
\hline $\begin{array}{l}\text { History of diagnostic } \\
\text { classification, } \mathrm{n}(\%)^{a}\end{array}$ & $55(23.3)$ & $19(23.2)$ & $11(16.4)$ & $25(28.7)$ \\
\hline
\end{tabular}

Note. ACT, acceptance and commitment therapy; EW, expressive writing; WLC, waiting-list control.

${ }^{a}$ No significant difference between intervention and control condition $(p>0.05)$.

b Significant difference between intervention and control condition $(p<0.05)$.

c Owing to a computer error data on marital status, nationality and daily activities of 5 participants in the ACT group and 3 participants in the EW group were mistakenly deleted. 
Table 2 - Outcome measure scores and repeated measures analysis of variance.

\begin{tabular}{|c|c|c|c|c|c|c|c|c|c|c|}
\hline \multirow[b]{3}{*}{ CES-D } & \multirow[b]{3}{*}{$\mathrm{ACT}$} & \multicolumn{8}{|c|}{ Score, mean (sd) } & \multirow{3}{*}{$\begin{array}{l}F^{a} \\
3.07^{*}\end{array}$} \\
\hline & & \multicolumn{2}{|c|}{ Pre-treatment } & \multicolumn{2}{|c|}{ Post-treatment } & \multicolumn{2}{|c|}{ Follow-up 1} & \multicolumn{2}{|c|}{ Follow-up 2} & \\
\hline & & 26.70 & 8.02 & 14.68 & 8.05 & 14.42 & 9.78 & 15.07 & 8.66 & \\
\hline & EW & 27.07 & 9.17 & 17.51 & 7.87 & 16.06 & 8.78 & 14.96 & 9.59 & \\
\hline & WLC & 26.51 & 8.16 & 19.34 & 8.55 & 17.71 & 10.72 & & & \\
\hline \multirow[t]{3}{*}{ HADS-A } & $\mathrm{ACT}$ & 8.73 & 3.01 & 6.15 & 3.25 & 6.49 & 3.57 & 6.28 & 3.50 & $3.50^{* *}$ \\
\hline & EW & 9.20 & 2.86 & 7.47 & 3.23 & 7.22 & 3.60 & 6.78 & 3.44 & \\
\hline & WLC & 8.70 & 2.99 & 7.82 & 3.62 & 7.45 & 3.76 & & & \\
\hline \multirow[t]{3}{*}{ MHC-SF } & $\mathrm{ACT}$ & 2.47 & 0.76 & 3.03 & 0.89 & 3.05 & 0.95 & 3.13 & 0.94 & $2.70^{*}$ \\
\hline & EW & 2.36 & 0.75 & 2.74 & 0.75 & 2.83 & 0.83 & 2.90 & 0.86 & \\
\hline & WLC & 2.45 & 0.80 & 2.69 & 0.86 & 2.84 & 0.93 & & & \\
\hline \multirow[t]{3}{*}{ AAQ-II } & $\mathrm{ACT}$ & 40.66 & 8.07 & 47.74 & 9.24 & 47.97 & 10.28 & 48.86 & 10.28 & $4.95^{\star \star \star}$ \\
\hline & EW & 38.93 & 6.88 & 44.15 & 7.24 & 45.40 & 8.11 & 45.72 & 8.54 & \\
\hline & WLC & 40.37 & 9.37 & 43.04 & 9.60 & 43.82 & 10.11 & & & \\
\hline \multirow[t]{3}{*}{ FFMQ-obs } & $\mathrm{ACT}$ & 14.66 & 2.96 & 15.80 & 3.00 & 15.63 & 2.88 & 18.86 & 3.58 & 2.38 \\
\hline & EW & 14.14 & 2.83 & 15.37 & 2.54 & 15.37 & 2.38 & 18.30 & 2.87 & \\
\hline & WLC & 14.61 & 3.18 & 14.99 & 3.10 & 15.33 & 3.01 & & & \\
\hline \multirow[t]{3}{*}{ FFMQ-des } & $\mathrm{ACT}$ & 17.28 & 3.68 & 18.67 & 3.33 & 18.51 & 3.80 & 15.73 & 3.00 & $3.76^{\star \star \star}$ \\
\hline & EW & 16.81 & 3.46 & 17.69 & 3.47 & 18.16 & 3.25 & 15.21 & 2.47 & \\
\hline & WLC & 17.70 & 3.22 & 17.69 & 3.85 & 17.84 & 3.90 & & & \\
\hline \multirow[t]{3}{*}{ FFMQ-act } & ACT & 15.44 & 3.74 & 15.66 & 2.60 & 16.64 & 3.49 & 16.49 & 3.59 & 1.16 \\
\hline & EW & 14.67 & 3.25 & 15.41 & 2.85 & 15.80 & 3.07 & 15.84 & 3.56 & \\
\hline & WLC & 15.14 & 3.69 & $15 \cdot 30$ & 3.81 & 15.70 & 3.80 & & & \\
\hline \multirow[t]{3}{*}{ FFMQ-nj } & $\mathrm{ACT}$ & 13.84 & 3.41 & 16.21 & 3.41 & 16.21 & 3.51 & 16.63 & 3.73 & $3.80^{\star \star}$ \\
\hline & EW & 13.71 & 3.13 & 15.14 & 2.82 & 15.30 & 2.85 & 15.53 & 3.25 & \\
\hline & WLC & 13.84 & 3.72 & 14.77 & 4.29 & 14.44 & 3.79 & & & \\
\hline \multirow[t]{3}{*}{ FFMQ-nr } & $\mathrm{ACT}$ & 13.94 & 3.41 & 16.83 & 2.57 & 16.72 & 3.16 & 16.80 & 3.02 & $8.94^{* * *}$ \\
\hline & EW & 14.37 & 3.06 & 15.90 & 2.59 & 15.98 & 2.55 & 16.15 & 2.65 & \\
\hline & WLC & 14.38 & 2.78 & 14.69 & 3.39 & 15.11 & 3.26 & & & \\
\hline
\end{tabular}

Note. AAQ-II, Acceptance and Action Questionnaire-II; ACT, acceptance and commitment therapy; CES-D, Center for Epidemiologic Studies - Depression scale; EW, expressive writing; FFMQ, Five Facet Mindfulness Questionnaire (obs, observing; des, describing; act, acting with awareness; nj, non-judging of inner experience; $n r$, non-reactivity to inner experience); HADS-A, Hospital Anxiety and Depression Scale - Anxiety subscale; MHC-SF, Mental Health Continuum - Short Form; WLC, waiting-list control.

a Time $x$ group analysis of variance.

${ }^{*} p<0.05,{ }^{* *} p<0.01,{ }^{* * *} p<0.001$. 


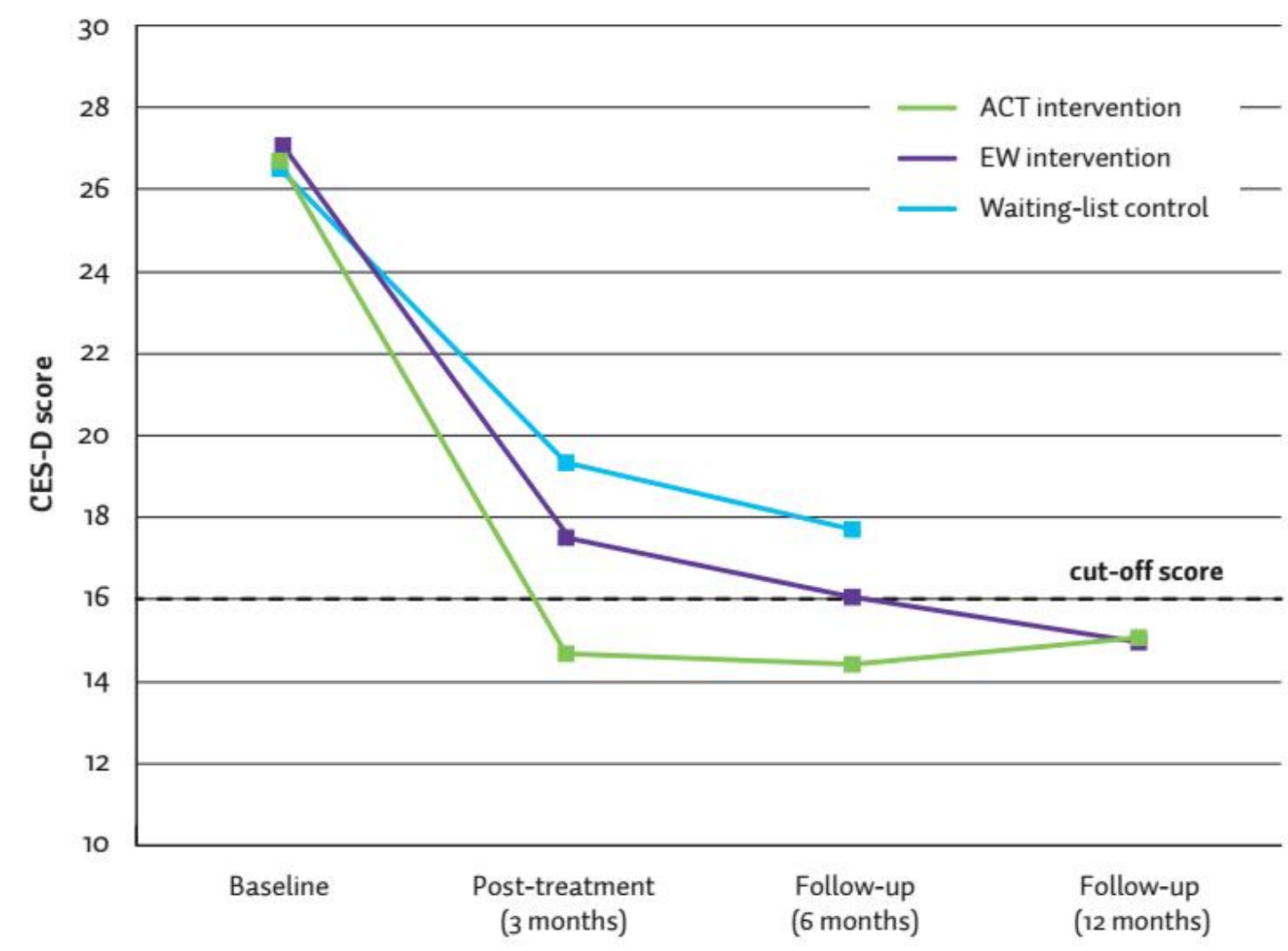

Figure 2 - Time x group repeated measures analysis of variance on Center for Epidemiologic StudiesDepression scale (CES-D).

Note. ACT, acceptance and commitment therapy; EW, expressive writing.

In Table 3 the effect sizes between the conditions at post-intervention $\left(T_{1}\right)$ and 6-month follow-up $\left(T_{2}\right)$ are presented. At $T_{1}$ moderate and small effect sizes were found on all the outcome measures for the ACT intervention compared with the waiting-list condition, and small effect sizes compared with the expressive writing intervention. For the latter intervention small effect sizes were found compared with the waiting-list condition. At $\mathrm{T}_{2}$ small effect sizes were found on all the outcome measures for the ACT intervention compared with the other two conditions. The expressive writing intervention also showed small effect sizes compared with the waiting-list condition.

\section{Effect maintenance}

Repeated measures ANOVA revealed no significant difference between the ACT and expressive writing interventions on the change in outcome measures from $T_{1}$ to $T_{3}$. Also, within-group analyses showed no significant change for both interventions on all outcome measures. Overall, both interventions showed maintenance of the effects on the primary and secondary measures from 6 months to 12 months of follow-up. 
Table 3 - Effect sizes (Cohen's d) between conditions post-treatment and at 6-month follow-up.

\section{Post-treatment 6-month follow-up \\ ACT-WLC ACT-EW EW-WLC ACT-WLC ACT-EW EW-WLC}

Primary outcome

$\begin{array}{lllllll}\text { CES-D } & 0.56 & 0.36 & 0.22 & 0.32 & 0.18 & 0.17\end{array}$

Secondary outcomes

HADS-A

0.49

0.41

0.10

0.26

0.20

0.06

MHC-SF

0.39

0.35

0.06

0.22

0.25

0.01

AAQ-II

0.50

0.43

0.13

0.41

0.28

0.17

FFMQ-SF

0.50

0.38

0.19

0.43

0.29

0.20

Note. AAQ-II, Acceptance and Action Questionnaire-II; ACT, acceptance and commitment therapy; CES-D, Center for Epidemiologic Studies - Depression scale; EW, expressive writing; FFMQ-SF, Five Facet Mindfulness Questionnaire - Short Form; HADS-A, Hospital Anxiety and Depression Scale Anxiety subscale; MHC-SF, Mental Health Continuum - Short Form; WLC, waiting-list control.

\section{Clinically significant change}

The reliable change on the CES-D turned out to be a pre-treatment to post-treatment difference of at least 7 scale points. The proportion of participants who reached a clinically significant change post-treatment on the CES-D in the ACT intervention was $54 \%(n=44)$ versus $26 \%(n=23)$ in the waiting-list control group $(O R=3.22,95 \%$ CI 1.69 $-6.14, p<0.001 ; N N T=3.7)$. In the expressive writing intervention $21(31 \%)$ reached a clinically significant change, resulting in a non-significant difference compared with the waiting-list control $(p=0.50)$. Comparison of the two interventions on clinically significant change post-treatment on the CES-D resulted in a significant difference in favour of $\mathrm{ACT}(\mathrm{OR}=2.54,95 \% \mathrm{CI} 1.29-4.98$, p < 0.01; NNT = 4.5). At 6-month follow-up no significant difference was found for the proportion of participants who reached a clinically significant change on the CES-D in the ACT ( $52 \%, n=43)$ and expressive writing interventions $(36 \%, n=24)$ versus the waiting-list group $(44 \%, n=38)$. Comparison of the two interventions on clinically significant change at follow-up resulted in a significant difference in favour of $\mathrm{ACT}(\mathrm{OR}=1.98,95 \% \mathrm{CI} 1.02-3.83, \mathrm{p}<0.05 ; \mathrm{NNT}=6.0)$.

\section{Effects on diagnostic classification}

Table 4 presents the results of the proportions of success for the MINI diagnoses $(n=204)$. Logistic regressions on the proportions of success were all non-significant, showing similar results of favourable outcome on the MINI. Chi-squared 
tests revealed significant proportional improvement in the diagnosis of recurrent depression for both $\mathrm{ACT}\left(\mathrm{X}^{2}(1, \mathrm{n}=152)=6.03, \mathrm{P}<0.01\right)$ and the expressive writing intervention $\left(X^{2}(1, n=132)=15.9, p<0.001\right)$ compared with the waiting-list condition. Comparison of the two interventions on proportions of improvement in the diagnosis of recurrent depression was non-significant $\left(X^{2}(1, n=124)=2.83, p=0.09\right)$.

Table 4 - Improvement according to diagnostic category ${ }^{a}$.

Number participants improved

\begin{tabular}{|c|c|c|c|c|c|c|}
\hline \multirow[b]{2}{*}{ Diagnosis } & \multicolumn{2}{|c|}{$\begin{array}{l}\text { ACT } \\
(n=72)\end{array}$} & \multicolumn{2}{|c|}{$\begin{array}{l}\text { EW } \\
(n=52)\end{array}$} & \multicolumn{2}{|c|}{$\begin{array}{l}\text { WLC } \\
(n=80)\end{array}$} \\
\hline & $T_{0}$ & $\mathrm{~T}_{2}$ & $T_{0}$ & $\mathrm{~T}_{2}$ & $\mathrm{~T}_{\mathrm{o}}$ & $\mathrm{T}_{2}$ \\
\hline No diagnosis & 36 & 51 & 22 & 40 & 39 & 49 \\
\hline Major depressive episode & 7 & 2 & 6 & 0 & 5 & 1 \\
\hline Recurrent depression & 21 & 7 & 21 & 4 & 19 & 14 \\
\hline Dysthymic disorder & ० & 1 & 3 & o & 2 & ० \\
\hline Other mood disorders & 2 & 2 & 4 & 1 & 5 & 2 \\
\hline Anxiety disorder & 16 & 9 & 11 & 7 & 17 & 14 \\
\hline
\end{tabular}

Note. ACT, acceptance and commitment therapy; EW, expressive writing; $T_{0}$, baseline; $T_{2}, 6$-month follow-up; WLC, waiting-list control.

a As measured by the Mini International Neuropsychiatric Interview and the Sheehan Disability Scale at $T_{0}$ and $T_{2}$ based on per protocol analysis $(n=204 ; 86 \%)$.

\section{Discussion}

This is the first study to investigate the effects of a web-based ACT intervention for adults with mild to moderate depressive symptoms in a randomised controlled trial. The effects of the intervention were compared with a waiting list and an active control condition and were measured at post-treatment and at 6-month and 12-month follow-up. Overall, the results show that in the short term the ACT intervention was significantly more effective on the primary outcome measure and most of the secondary outcome measures than both the waiting-list control and the expressive writing intervention, but that both interventions had similar effects on depressive symptoms and secondary measures after 6 months and 12 months.

In comparison with the waiting-list control, participants receiving ACT showed significantly larger reductions of depressive symptoms post-treatment. The effect 
size at that point was moderate and at the 6-month follow-up it was small. The likelihood of a clinically significant change in depressive symptoms at the post-treatment assessment was substantially higher in the ACT group, compared with both the waiting-list (NNT = 3.7) and the expressive writing groups (NNT = 4.5). Moreover, comparison of the expressive writing and waiting-list control groups resulted in a non-significant difference. This outcome suggests that ACT was superior to both the active intervention and the waiting-list control in directly decreasing clinically relevant depressive symptoms. This is relevant, as the presence of such symptoms is the most important risk factor for major depressive disorder and is associated with considerable economic costs (Cuijpers et al., 2007). Moreover, the ACT intervention resulted in significantly larger post-treatment reductions in anxiety and improvements in positive mental health in comparison with the waiting-list condition. The effect sizes were in the small range at post-treatment and in the small (non-significant) range at 6-month follow-up.

\section{Interpretation of the findings}

The findings at post-treatment are in line with findings from other studies of web-based interventions for adult depression (Arnberg et al., 2014; Richards and Richardson, 2012). The smaller effects at 6-month follow-up appear to be largely due to further recovery in the waiting-list condition. An explanation could be that the waiting-list group anticipated starting the intervention directly after filling in the questionnaire at follow-up. The maintenance of the effect in the ACT intervention is similar to findings in earlier studies about the effect of ACT on depression (Bohlmeijer et al., 2011b; Fledderus et al., 2012a). Furthermore, this study shows that the effects of ACT were maintained at 12-month follow-up. Our results from the diagnostic classification analysis showed no significant difference in the proportions of success, meaning that in all conditions fewer participants were diagnosed with a mood disorder post-treatment. However, additional analyses showed a significant difference for both interventions on the diagnosis of recurrent depression compared with the waiting-list control. This finding suggests that both interventions may be especially effective for people with recurrent depression, but this needs to be interpreted with caution as our study was not powered for this. For the ACT intervention a possible explanation could be that mindfulness is a substantial component of the therapy. In all lessons participants are invited to meditate in order to increase awareness and acceptance of negative emotions and thoughts. It has been shown that meta-cognitive awareness, i.e. seeing thoughts as mental events that come and go, is an important process contributing to the prevention of the recurrence of depression in people with a history of depression (Teasdale et al., 2002). As the participants in the ACT intervention showed a larger increase in mindfulness in comparison with the 
waiting list condition, this could explain the differential effects on recurrent depression at 6-month follow-up. However, these results must be interpreted with care and need to be supported by more research.

\section{Effectiveness of expressive writing}

Although it was found that the ACT intervention had larger post-treatment effects for depressive symptoms than the expressive writing intervention, no difference was found at follow-up. This was contrary to our hypothesis, in which we expected ACT to have a larger treatment effect both post treatment and at follow-up. One explanation could be that the attention and emotional support through email contact in both interventions contributed to the effects. Another explanation is that expressive writing is also an effective treatment. Expressive writing has often been applied as an intervention, in which people write about emotional events on at least 3 days a week for 15-30 min for 3 or 4 consecutive days (Pennebaker, 1997). However, to make the format more equal to the ACT intervention the intervention was extended to 9 weeks. To enhance adherence to this longer intervention many components were added, one of which was psycho-education on emotion regulation based on Gross's model. We found significant improvements from baseline to 6-month follow-up for the expressive writing group in psychological flexibility and the mindfulness facets describing, non-judging of inner experience and non-reactivity to inner experience compared with the waiting-list control. Writing about negative emotions for many weeks and writing about positive events may help people to regulate emotions in a way that is similar to ACT, i.e. diminishing avoidance and increasing acceptance of emotions. Some authors have suggested that emotional writing may yield larger effects (Nazarian and Smyth, 2013; Niles et al., 2014), but there is no consensus on the mechanisms of change. Our findings suggest that a comprehensive expressive writing intervention could be an effective web-based intervention, but more research is needed to support this.

\section{Adherence}

Adherence to treatment was high, with only 13 out of 82 (16\%) participants in the ACT group attending fewer than six sessions and $73 \%$ completing all nine sessions. The web-based intervention was designed using the Center for eHealth Research and Disease Management road map to achieve a user-friendly application that fits the values of the stakeholders and to evaluate the process of development (Kelders et al., 2013). The high level of adherence in our study may indicate feasibility and compatibility with the wishes of our participants. Furthermore, effort was made to include persuasive technology in the design of the web-based interventions, as persuasive design has been shown to be positively related to adherence (Kelders et al., 2012). 


\section{Strengths and limitations}

A strength of our study is that the ACT intervention was compared not only with a waiting-list condition but also with an active control condition in the form of a web-based expressive writing intervention. Non-specific treatment factors such as attention and emotional support are generally recognised to be important for therapeutic effect (Martin et al., 2000). Also, inclusion criteria were kept broad to enhance generalisability with respect to the general population and the external validity of the study. Moreover, adding semi-structured interviews as diagnostic measures enhanced the reliability of the outcome measures. Finally, high attrition rates are common in studies of web-based interventions (Eysenbach, 2005). In our study treatment adherence was as high as $84 \%$, in contrast to many previous internet studies that showed lower adherence rates (Christensen et al., 2009).

Some limitations also apply. The first and most important is that the recruitment strategy raises the possibility of self-selection bias (i.e. self-referral and motivation for time investment). This, and the fact that our participants tended to have high levels of education relative to the general public, raise questions on generalisability. It is a common finding in internet studies that highly educated women are especially prone to apply for guided web-based self-help interventions (Eysenbach, 2005). Second, there was no assessment of interrater reliability of the diagnostic classification in this study. However, previous studies have provided justification for this method of assessing psychiatric disorders (Evans et al., 2004). A third limitation is the absence of competence measures of the counsellors because of the risk of not following the treatment protocol. However, all counsellors were supervised and the treatment protocol was highly standardised. Fourth, although we standardised the interventions to make them equivalent, the format of the interventions differed somewhat between treatments. The expressive writing intervention relied almost exclusively on text-based material, whereas the ACT used more experiential exercises (mindfulness exercises and interactive material) and relied more on a mix of text-based and picture-based material, which is in line with the theoretical underpinnings of the ACT model (Hayes et al., 2006; Hayes et al., 2012).

\section{Study implications}

The clinical implications of this trial are that ACT can be effective as a web-based public mental health intervention for people with mild to moderate depressive symptoms, at least for women with medium to high levels of education. Although ACT was found to have larger effects than expressive writing in the short term, our findings also suggest that a comprehensive web-based expressive writing therapy might be a promising public mental health intervention. This needs to be corroborated in future studies. 


\section{References}

Aldao, A. \& Nolen-Hoeksema, S. (2010). Specificity of cognitive emotion regulation strategies: A transdiagnostic examination. Behaviour Research and Therapy 48, 974-983.

Andersson, G. \& Cuijpers, P. (2008). Pros and cons of online cognitive-behavioural therapy. British Journal of Psychiatry 193, 270-271.

Arnberg, F. K., Linton, S. J., Hultcrantz, M., Heintz, E. \& Jonsson, U. (2014). Internet-delivered psychological treatments for mood and anxiety disorders: A systematic review of their efficacy, safety, and cost-effectiveness. PLoS ONE 9, eg8118.

Baer, R. A., Smith, G. T., Hopkins, J., Krietemeyer, J. \& Toney, L. (2006). Using self-report assessment methods to explore facets of mindfulness. Assessment 13, 27-45.

Beekman, A. T. F., Deeg, D. J. H., Van Limbeek, J., Braam, A. W., De Vries, M. Z. \& Van Tilburg, W. (1997). Criterion validity of the Center for Epidemiologic Studies Depression scale (CES-D): Results from a community-based sample of older subjects in the Netherlands. Psychological Medicine 27, 231-235.

Bijl, R. V., Ravelli, A. \& Van Zessen, G. (1998). Prevalence of psychiatric disorder in the general population: Results of the Netherlands Mental Health Survey and Incidence Study (NEMESIS). Social Psychiatry and Psychiatric Epidemiology 33, 587-595.

Bohlmeijer, E. \& Hulsbergen, M. (2013). A beginner's guide to mindfulness: live in the moment. Open University Press: Oxford.

Bohlmeijer, E., Ten Klooster, P. M., Fledderus, M., Veehof, M. \& Baer, R. (2011a). Psychometric properties of the five facet mindfulness questionnaire in depressed adults and development of a short form. Assessment 18, 308-320.

Bohlmeijer, E. T., Fledderus, M., Rokx, T. A. J. J. \& Pieterse, M. E. (2011b). Efficacy of an early intervention based on acceptance and commitment therapy for adults with depressive symptomatology: Evaluation in a randomized controlled trial. Behaviour Research and Therapy 49, 62-67.

Bond, F. W., Hayes, S. C., Baer, R. A., Carpenter, K. M., Guenole, N., Orcutt, H. K., ... Zettle, R. D. (2011). Preliminary Psychometric Properties of the Acceptance and Action Questionnaire-II: A Revised Measure of Psychological Inflexibility and Experiential Avoidance. Behavior Therapy 42, 676-688.

Cavanagh, K., Strauss, C., Forder, L. \& Jones, F. (2014). Can mindfulness and acceptance be learnt by self-help?: A systematic review and meta-analysis of mindfulness and acceptance-based self-help interventions. Clinical Psychology Review 34, 118-129.

Christensen, H., Griffiths, K. M. \& Farrer, L. (2009). Adherence in internet interventions for anxiety and depression. Journal of Medical Internet Research 11, e13.

Cohen, J. A. (1992). Power primer. Psychological Bulletin 112, 155-159.

Cook, R. J. \& Sackett, D. L. (1995). The number needed to treat: A clinically useful measure of treatment effect. British Medical Journal 310, 452-454.

Cuijpers, P. \& Smit, F. (2004). Subthreshold depression as a risk indicator for major depressive disorder: A systematic review of prospective studies. Acta Psychiatrica Scandinavica 109, 325-331.

Cuijpers, P., Smit, F., Oostenbrink, J., De Graaf, R., Ten Have, M. \& Beekman, A. (2007). Economic costs of minor depression: A population-based study. Acta Psychiatrica Scandinavica 115, 229-236.

Cuijpers, P., Van Straten, A., Warmerdam, L. \& Van Rooy, M. J. (2010). Recruiting participants for interventions to prevent the onset of depressive disorders: Possibile ways to increase participation rates. BMC Health Services Research 10, 181.

Dempster, A. P., Laird, N. M. \& Rubin, D. B. (1977). Maximum likelihood from incomplete data via the EM algorithm. Journal of the Royal Statistical Society Series B Methodological 39, 1-38.

Evans, M., Kessler, D., Lewis, G., Peters, T. J. \& Sharp, D. (2004). Assessing mental health in primary care research using standardized scales: Can it be carried out over the telephone? Psychological Medicine 34, 157-162. 
Eysenbach, G. (2005). The law of attrition. Journal of Medical Internet Research 7, en1.

Fledderus, M., Bohlmeijer, E. T., Pieterse, M. E. \& Schreurs, K. M. G. (2012a). Acceptance and commitment therapy as guided self-help for psychological distress and positive mental health: A randomized controlled trial. Psychological Medicine 42, 485-495.

Fledderus, M., Oude Voshaar, M. A. H., Ten Klooster, P. M. \& Bohlmeijer, E. T. (2012b). Further evaluation of the psychometric properties of the acceptance and action questionnaire-II. Psychological Assessment 24, 925-936.

Frattaroli, J. (2006). Experimental disclosure and its moderators: A meta-analysis. Psychological Bulletin 132, 823-865.

Gross, J. J. \& Thompson, R. A. (2007). Emotion regulation: conceptual foundations. In Handbook of Emotion Regulation (ed. J. J. Gross), pp. 3-24. Guilford Press: New York.

Haringsma, R., Engels, G. I., Beekman, A. T. F. \& Spinhoven, P. (2004). The criterion validity of the Center for Epidemiological Studies Depression Scale (CES-D) in a sample of self-referred elders with depressive symptomatology. International Journal of Geriatric Psychiatry 19, 558-563.

Hayes, S. C., Luoma, J. B., Bond, F. W., Masuda, A. \& Lillis, J. (2006). Acceptance and Commitment Therapy: Model, processes and outcomes. Behaviour Research and Therapy 44, 1-25.

Hayes, S. C., Strosahl, K. D. \& Wilson, K. G. (2012). Acceptance and Commitment Therapy: the Process and Practice of Mindful Change. Guilford Press: New York.

Jacobson, N. S. \& Truax, P. (199). Clinical significance: A statistical approach to defining meaningful change in psychotherapy research. Journal of Consulting and Clinical Psychology 59, 12-19.

Kabat-Zinn, J. (1990). Full catastrophe living: Using the wisdom of your body and mind to face stress, pain and illness. Delacorte: New York.

Kashdan, T. B. \& Rottenberg, J. (2010). Psychological flexibility as a fundamental aspect of health. Clinical Psychology Review 30, 865-878.

Kelders, S. M., Kok, R. N., Ossebaard, H. C. \& Van Gemert-Pijnen, J. E. W. C. (2012). Persuasive system design does matter: A systematic review of adherence to web-based interventions. Journal of Medical Internet Research 14, e152.

Kelders, S. M., Pots, W. T., Oskam, M. J., Bohlmeijer, E. T. \& Van Gemert-Pijnen, J. E. (2013). Development of a web-based intervention for the indicated prevention of depression. BMC Medical Informatics and Decision Making 13, 1-11.

Keyes, C. L. M., Wissing, M., Potgieter, J. P., Temane, M., Kruger, A. \& van Rooy, S. (2008). Evaluation of the mental health continuum-short form (MHC-SF) in Setswana-speaking South Africans. Clinical Psychology and Psychotherapy 15, 181-192.

Lamers, S. M., Westerhof, G. J., Bohlmeijer, E. T., Ten Klooster, P. M. \& Keyes, C. L. (2011). Evaluating the psychometric properties of the mental health Continuum-Short Form (MHC-SF). Journal of Clinical Psychology 67, 99-110.

Leon, A. C., Olfson, M., Portera, L., Farber, L. \& Sheehan, D. V. (1997). Assessing psychiatric impairment in primary care with the Sheehan Disability Scale. International Journal of Psychiatry in Medicine 27, 93-105.

Martin, D. J., Garske, J. P. \& Katherine Davis, M. (2000). Relation of the therapeutic alliance with outcome and other variables: A meta-analytic review. Journal of Consulting and Clinical Psychology $68,438-450$.

Moher, D., Schulz, K. F., Altman, D. G. \& Lepage, L. (2001). The CONSORT statement: Revised recommendations for improving the quality of reports of parallel-group randomized trials. Annals of Internal Medicine 134, 657-662.

Morris, S. B. \& DeShon, R. P. (2002). Combining effect size estimates in meta-analysis with repeated measures and independent-groups designs. Psychological Methods 7, 105-125.

Muñoz, R. F., Cuijpers, P., Smit, F., Barrera, A. Z. \& Leykin, Y. (2010). Prevention of major depression. Annual Review of Clinical Psychology 6, 181-212.

Nazarian, D. \& Smyth, J. M. (2013). An experimental test of instructional manipulations in expressive writing interventions: Examining processes of change. Journal of Social and Clinical Psychology $32,71-96$. 
Niles, A. N., Haltom, K. E. B., Mulvenna, C. M., Lieberman, M. D. \& Stanton, A. L. (2014). Randomized controlled trial of expressive writing for psychological and physical health: The moderating role of emotional expressivity. Anxiety, Stress and Coping 27, 1-17.

Pennebaker, J. W. (1997). Writing about emotional experiences as a therapeutic process. Psychological Science 8, 162-166.

Radloff, L. S. (1977). The CES-D scale, a self report depression scale for reseach in the general population. Applied Psychological Measurement 1, 385-401.

Richards, D. \& Richardson, T. (2012). Computer-based psychological treatments for depression: A systematic review and meta-analysis. Clinical Psychology Review 32, 329-342.

Sheehan, D. V., Lecrubier, Y., Sheehan, K. H., Amorim, P., Janavs, J., Weiller, E., ... Dubar, G. C. (1998). The Mini-International Neuropsychiatric Interview (M.I.N.I.): the development and validation of a structured diagnostic psychiatric interview for DSM-IV and ICD-10. Journal of Clinical Psychiatry 59, 22-33.

Smit, F., Ederveen, A., Cuijpers, P., Deeg, D. \& Beekman, A. (2006). Opportunities for cost-effective prevention of late-life depression: An epidemiological approach. Archives of General Psychiatry 63, 290-296.

Spinhoven, P., Ormel, J., Sloekers, P. P. A., Kempen, G. I. J. M., Speckens, A. E. M. \& Van Hemert, A. M. (1997). A validation study of the hospital anxiety and depression scale (HADS) in different groups of Dutch subjects. Psychological Medicine 27, 363-370.

Teasdale, J. D., Moore, R. G., Hayhurst, H., Pope, M., Williams, S. \& Segal, Z. V. (2002). Metacognitive awareness and prevention of relapse in depression: Empirical evidence. Journal of Consulting and Clinical Psychology 70, 275-287.

Van Vliet, I. M. \& De Beurs, E. (2007). The MINI-International Neuropsychiatric Interview (M.I.N.I.). A brief structured diagnostic psychiatric interview for DSM-IV and ICD-10 psychiatric disorders [in Dutch]. Tijdschrift voor Psychiatrie 49, 393-397.

Zigmond, A. S. \& Snaith, R. P. (1983). The hospital anxiety and depression scale. Acta Psychiatrica Scandinavica $67,367-370$. 


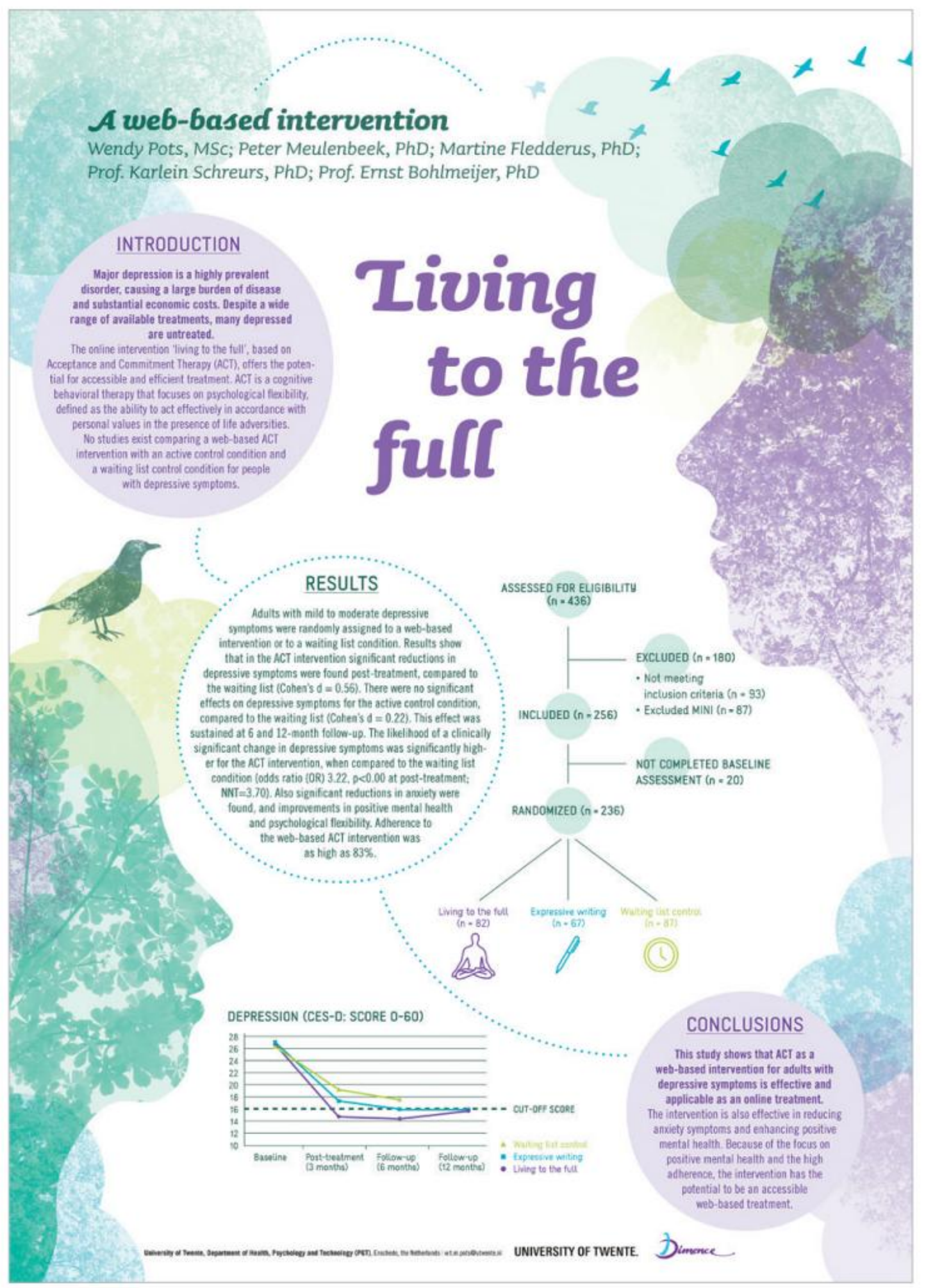

Note. RINO groep posterprijs, KNP congres, 14 april 2014. 
CHAPTER 5 


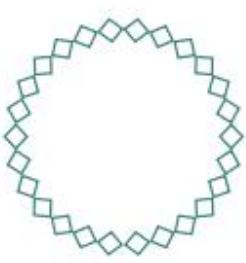

0

$0 \varnothing$

$0 \varnothing$

00

$0 \varnothing$

$0 \varnothing$

CHAPTER 6 


\section{How and for whom does}

web-based Acceptance and

Commitment Therapy work?

Mediation and moderation

analyses of web-based ACT for

depressive symptoms

Pots, W.T.M.,Trompetter, H.R., Schreurs, K.M.G. \& Bohlmeijer, E.T. (2016).

How and for whom does web-based Acceptance and Commitment Therapy work?

Mediation and moderation analyses of web-based ACT for depressive symptoms.

BMC Psychiatry 16,158 


\section{Abstract}

Acceptance and Commitment Therapy (ACT) has been demonstrated to be effective in reducing depressive symptoms. However, little is known how and for whom therapeutic change occurs, specifically in web-based interventions. This study focuses on the mediators, moderators and predictors of change during a web-based ACT intervention. Data from 236 adults from the general population with mild to moderate depressive symptoms, randomised to either web-based ACT $(n=82)$ or one of two control conditions (web-based Expressive Writing (EW; $n=67)$ and a waiting list $(n=$ 87)), were analysed. Single and multiple mediation analyses, and exploratory linear regression analyses were performed using PROCESS and linear regression analyses, to examine mediators, moderators and predictors on pre- to post- and follow-up treatment change of depressive symptoms. The treatment effect of ACT versus the waiting list was mediated by psychological flexibility and two mindfulness facets. The treatment effect of ACT versus EW was not significantly mediated. The moderator analyses demonstrated that the effects of web-based ACT did not vary according to baseline patient characteristics when compared to both control groups. However, higher baseline depressive symptoms and positive mental health and lower baseline anxiety were identified as predictors of outcome across all conditions. Similar results were found for follow-up. The findings of this study corroborate the evidence that psychological flexibility and mindfulness are distinct process mechanisms that mediate the effects of web-based ACT intervention. The results indicate that there are no restrictions to the allocation of web-based $\mathrm{ACT}$ intervention and that web-based ACT can work for different subpopulations. 


\section{Background}

Acceptance and Commitment Therapy (ACT) is an empirically based behavioural cognitive therapy that uses acceptance and mindfulness strategies together with commitment and behaviour change strategies, to increase the ability to act in accordance with personal values in the presence of life adversities (Hayes et al., 2006; Hayes et al., 2012). The effectiveness of ACT in reducing depressive symptoms has been established in several recent meta-analyses (A-Tjak et al., 2015; Öst, 2014; Ruiz, 2010). In addition, there is growing evidence for the efficacy of web-based ACT interventions (Bricker et al., 2013; Buhrman et al., 2013; Hesser et al., 2012; Lappalainen et al., 2014; Pots et al., 2015; Trompetter et al., 2014). Besides evaluating the efficacy and effectiveness of psychological treatments, randomised controlled trials can be valuable in revealing how (i.e. mediators) and for whom (i.e. moderators) therapeutic change occurs. Mediators and moderators are important for the further optimization of treatments and their clinical and cost-effectiveness. Specifically, knowledge of mediators of change enables treatment components to be included that are crucial to recovery (Kazdin, 2007). Furthermore, both nonspecific treatment predictors and specific treatment moderators indicate who benefits from treatment, or under which conditions treatment works best (Kraemer et al., 2002). Moderators and predictors can help tailor interventions to different subpopulations with possibly different causal mechanisms of disorders and ultimately improve clinical decision about treatment (Kraemer et al., 2002; MacKinnon, 2011). Research into potential mediators and moderators of action in web-based ACT interventions is, however, in its infancy. The focus of this study is therefore on the possible mediators, moderators and predictors of change during a web-based ACT intervention.

The central therapeutic mechanism in ACT is psychological flexibility, which is the ability to act in accordance with intrinsically motivating values or goals while being in contact with the present moment (Hayes et al., 2006; Hayes et al., 2012). People who are psychological flexible also score high on acceptance, which is seen as a more effective strategy for regulating negative emotions and thoughts than experiential avoidance, i.e. the persistent and generally fruitless attempts to avoid unwanted private experiences such as feelings, thoughts, and bodily sensations (Aldao and Nolen-Hoeksema, 2010; Hayes et al., 2006; Kashdan and Rottenberg, 2010). In the model underlying ACT, psychological flexibility is defined by six interrelated therapeutic processes: acceptance, cognitive defusion, contact with the present moment, self-as-context, values, and committed action. Mindfulness is taught in the context of the first four processes of the ACT model, where exercises are used to enhance an observing and non-judging self, together with the awareness and acceptance of unwanted private experiences such as thoughts and feelings. Mindfulness is 
often referred to as intentionally paying attention to present moment experiences in a non-judgemental way (Kabat-Zinn, 1990). Mindfulness, as measured by the Five Facet Mindfulness Questionnaire (FFMQ), is conceptualized in several facets, which are observing (noticing or attending to internal and external experiences), describing (labelling internal experiences with words), acting with awareness (attending to one's activities of the moment), non-judging of inner experience (taking a non-evaluative stance toward thoughts and feelings), and non-reactivity to inner experience (allowing thoughts and feelings to come and go). Baer et al. (2006) stated that non-reactivity and non-judging may be seen as ways of operationalizing acceptance. They found a correlation of $r=.49$ between the Acceptance and Action Questionnaire-II (AAQ-II; measuring psychological flexibility) and the non-judging facet of the FFMQ. Although the AAQ-II and FFMQ are not meant to measure the same construct, Fledderus et al. (2012b) found that the AAQ-II was positively related to mindfulness facets and positive mental health and negatively related to depression and anxiety. The mindfulness facets correlated significantly with the AAQ-II, with - in agreement with Bear (2006) - the strongest relation between AAQ-II and non-judging. The strongest relation between AAQ-II and non-judging $(r=.54)$ suggest that the AAQ-II and the non-judging facet of the FFMQ measure are related but distinct constructs. The results also showed that the AAQ-II explains additional variance in relevant outcomes such as depression, anxiety, and positive mental health. To date, there are several studies that have suggested that augmented mindfulness mediates the effects of an ACT intervention (e.g. Forman et al., 2012; Kocovski et al., 2013; McCracken and Gutiérrez-Martínez, 2011). Research into psychological flexibility as a mediator in ACT interventions confirms that psychological flexibility is a core component of the theoretical framework of ACT (e.g. Forman et al., 2012; Hayes et al., 2006; Kashdan and Rottenberg, 2010; Niles et al., 2014; Wicksell et al., 2011).

As opposed to face-to-face, web-based interventions offer advantages in availability and accessibility, but non-adherence or high drop-out is an issue (Christensen et al., 2009; Eysenbach, 2005). Some researchers have suggested that in web-based interventions specificity of effects can account for the higher drop-out rates that are common in web-based interventions (Christensen et al., 2009; MacKinnon, 2011). Given this higher specificity of effects and possible individual variability in self-support via web-based interventions, it is important to study moderators and predictors. Yet, little is known about the moderators of change of web-based ACT interventions, and research of moderator and predictor analyses of web-based ACT interventions for depressions is lacking. There is some limited research on moderators and predictors in face-to-face ACT interventions for anxiety, indicating that factors such as socio-demographic characteristics do not moderate or predict outcome, and that factors such as mood disorder comorbidity may be predictors and/or moderators (Davies et al., 2015; 
Niles et al., 2014; Wolitzky-Taylor et al., 2012). Also, a study by Flaxman and Bond (2010) on worksite stress management training (SMT), based on ACT, showed that the impact of SMT was significantly moderated by baseline distress. For mediators of change, the number of studies doing formal mediational analyses of psychological flexibility and mindfulness in web-based ACT interventions is small (Bricker et al., 2013; Trompetter et al., 2015). In the study of Trompetter et al. (2015) improvements in psychological flexibility mediated the effect of web-based ACT on psychological distress, further substantiating that psychological flexibility is a core component of the ACT model. Also, Bricker et al. (2013) found that acceptance processes mediated the effects on smoking cessation through greater acceptance of physical urges, cognitions, and emotions.

The aim of the current study was to identify mediators, moderators and predictors of participant improvement in the web-based ACT intervention in a recently published randomised controlled trial (RCT; Pots et al., 2015). The primary objective was to test the hypothesis that pre- to post-treatment changes in psychological flexibility and mindfulness mediated the effects of the web-based ACT intervention on depressive symptoms. A second objective was to explore which participant baseline characteristics and baseline symptoms moderated or predicted treatment effects. These latter analyses were considered exploratory given the lack of research so far.

\section{Methods}

\section{Participants and procedure}

The sample for the current study stems from the original sample in the RCT on the effectiveness of web-based public mental health intervention based on ACT (Pots et al., 2015). The study was approved by an independent medical ethics committee for research in mental health settings in The Netherlands (METiGG; number NL33619.097.100). In addition, this study has been recorded in the Dutch primary trial register for clinical trials (The Netherlands Trial Register, NTR2736). In brief, participants with mild to moderate depressive symptoms were recruited through advertisements in Dutch national newspapers and on the Internet. A webpage created for the purpose of this study included an outline of the study design, and a registration possibility for participation in the trial. Within the webpage candidates filled out a secured computerised informed consent. After receiving the informed consent, initial screening was conducted online for checking the in- and exclusion criteria by use of a self-report questionnaire in a fully automated computerised assessment battery. Study inclusion criteria were: a) an age of 18 years or older with mild to moderate depressive symptoms $>10$ on the Dutch version of the Center of Epidemiological 
Studies - Depression Scale (CES-D; Fledderus et al., 2012a), and b) completion of the baseline measurement. Applicants were excluded if on initial screening they reported: a) few depressive symptoms ( $\leq 10$ on the CES-D); b) received psychological or psychopharmacological treatment for mental complaints within the last three months; c) reading or writing problems due to insufficient Dutch language skills; and d) an inability to invest approximately 30 minutes per day up to three hours per week in the intervention and daily practices. Furthermore, participants with severe psychopathology were excluded when diagnosed with a current severe mental disorder or when having a moderate to high suicide risk, according to the Dutch version of the Mini International Neuropsychiatric Interview (Sheehan et al., 1998; Van Vliet and De Beurs, 2007) and the Sheehan Disability Scale (Leon et al., 1997).

Of the 436 persons that were assessed for eligibility, 236 participants were randomly allocated to either ACT $(n=82)$, an active control condition based on Expressive Writing $(E W ; n=67)$ or to a waiting list control condition (WLC; $n=87$ ). Figure 1 shows the CONSORT flow of the participants. Baseline characteristics can be found in Table 1 . Due to a programming error in the randomisation procedure, the number of participants in each condition differed. There were no significant differences at baseline between the conditions for any of the demographic variables or outcome measures, except for gender $\left[X^{2}(2, n=236)=22.78, p<0.00\right.$; percentage of female was higher in the ACT group, followed by WLC, followed by EW].

\section{Intervention}

In the current study an ACT-protocol called "Living to the Full” (Bohlmeijer and Hulsbergen, 2013) was used, which has an explicit focus on mindfulness (Bohlmeijer and Hulsbergen, 2013; Pots et al., 2015). The web-based ACT intervention comprised of nine online modules, which could be worked through in nine to twelve weeks. This self-help intervention has shown to be effective in reducing depressive and anxiety symptoms, and improving positive mental health, psychological flexibility and mindfulness, as a group course and as a bibliotherapy intervention with e-mail support (Bohlmeijer et al., 2011b; Fledderus et al., 2012a; Fledderus et al., 2010). The nine sessions are based on six core processes of ACT that together promote psychological flexibility (Hayes et al., 2012). Each module uses experiential exercises and metaphors to illustrate the ACT processes, text messages, tailored stories for motivation, and an option to personalise the homepage. Furthermore, participants were encouraged to practice daily mindfulness exercises that were provided on audio, downloadable within the web-based intervention. Weekly e-mail counselling by trained graduate psychology students was offered for personal feedback, encouragement and support. For a comprehensive description of the development of the intervention see Kelders et al. (2013). 


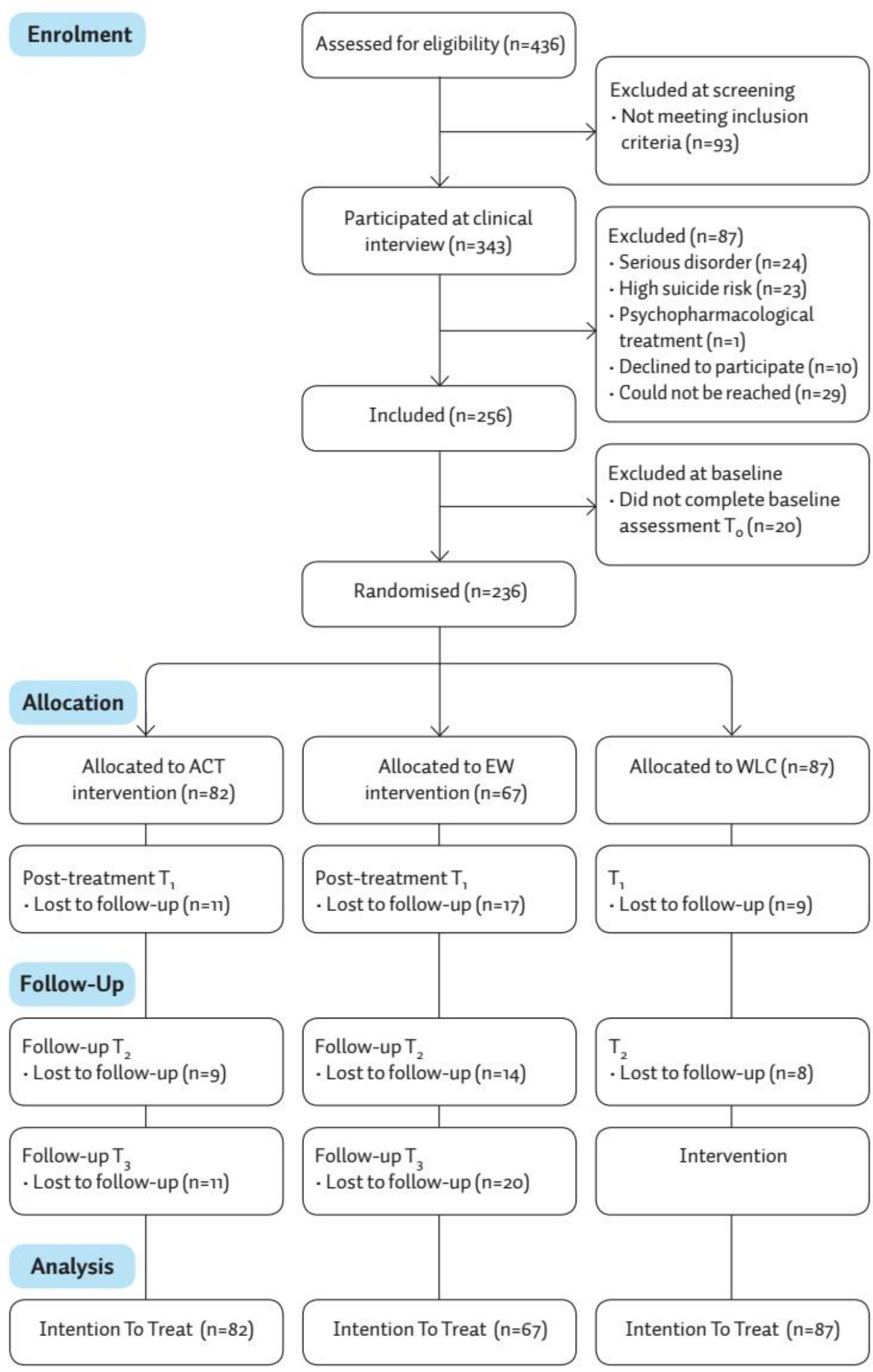

Figure 1 - CONSORT flow of the participants. 
Table 1 Baseline characteristics.

\begin{tabular}{|c|c|c|c|c|}
\hline Characteristics & $\begin{array}{l}\text { Total } \\
(n=236)\end{array}$ & $\begin{array}{l}\text { ACT } \\
(n=82)\end{array}$ & $\begin{array}{l}\text { EW } \\
(n=67)\end{array}$ & $\begin{array}{l}\text { WLC } \\
(n=87)\end{array}$ \\
\hline Age, mean, y (SD) & $46.85(12.06)$ & $45.15(10.78)$ & $46.73(12.65)$ & $48.54(12.63)$ \\
\hline Range & $20-73$ & $21-69$ & $20-69$ & $21-73$ \\
\hline \multicolumn{5}{|l|}{ Gender, No. (\%) } \\
\hline Female & $179(75.8)$ & $76(92.7)$ & $40(59.7)$ & $63(72.4)$ \\
\hline Male & $57(24.2)$ & $6(7 \cdot 3)$ & $27(40.3)$ & $24(27.6)$ \\
\hline \multicolumn{5}{|l|}{ Education, No. (\%) } \\
\hline High & $157(66.5)$ & $55(67.1)$ & $45(67.2)$ & $57(65.5)$ \\
\hline Middle & $75(31.8)$ & $24(29 \cdot 3)$ & $22(32.8)$ & $29(33.3)$ \\
\hline Low & $4(1.7)$ & $3(3.7)$ & $\circ(0.0)$ & $1(1.1)$ \\
\hline \multicolumn{5}{|l|}{ Diagnosis, No. (\%) } \\
\hline No diagnosis & $97(41.1)$ & $36(43.9)$ & $22(32.8)$ & $39(44.8)$ \\
\hline Major depressive episode & $18(7.6)$ & $7(8.6)$ & $6(9.0)$ & $5(5.8)$ \\
\hline Recurrent depression & $61(25.9)$ & $21(25.6)$ & $21(31.3)$ & $19(21.8)$ \\
\hline Dysthymic disorder & $5(2.1)$ & $o(0.0)$ & $3(4 \cdot 5)$ & $2(2.3)$ \\
\hline Other mood disorders & $11(4.7)$ & $2(2.4)$ & $4(6.0)$ & $5(5.8)$ \\
\hline Anxiety disorder & $44(18.6)$ & $16(19.5)$ & $11(16.4)$ & $17(19.5)$ \\
\hline \multicolumn{5}{|l|}{ Comorbidity, No. (\%) } \\
\hline Mood disorder & $54(22.9)$ & $22(26.8)$ & $17(25.4)$ & $15(17.2)$ \\
\hline Anxiety disorder & $3(1.3)$ & $o(0.0)$ & $3(4.5)$ & $0(0.0)$ \\
\hline
\end{tabular}

Note. $\mathrm{ACT}=$ Acceptance and Commitment Therapy, $\mathrm{EW}=$ Expressive Writing, $\mathrm{WLC}=$ Waiting List Condition.

The active control condition was a web-based Expressive Writing (EW) intervention based on Pennebaker's expressive writing paradigm (Pennebaker, 1997), and comprised of nine online sessions that could be worked through in nine to twelve weeks. Every session started with psycho-education on emotions and emotion regulation, and was followed by instructions of the method of EW. In general, people were instructed to write about emotional experiences for 15 to 30 minutes on at least three days during one week. E-mail counselling was offered similarly to the web-based ACT intervention.

Participants in the waiting list condition were offered no intervention and were free to access other forms of care. Six months after baseline these participants could start a web-based intervention of choice. 


\section{Summary of previously reported results from the RCT}

As reported in Pots et al. (2015), analyses using repeated measures ANOVA showed that the web-based ACT intervention was superior to both the active control condition (EW intervention) and the WLC, on all outcome measures (depressive symptoms, anxiety and positive mental health) and process variables (psychological flexibility and mindfulness, except for the mindfulness facets observing and acting with awareness). The effect sizes at post-treatment were small to moderate and ranged from 0.35 to 0.56 . The effects of the web-based ACT intervention were maintained at 6- and 12-month follow up. However, at follow-up there were no significant differences between the web-based ACT intervention and the web-based EW intervention or the WLC. Also, as there was no restricted access to care for the WLC, non-study treatment was checked but revealed no difference between the conditions. Overall, the results showed that on the short term the web-based ACT intervention was significantly more effective to both control conditions, but that both web-based interventions had similar effects on depressive symptoms and secondary measures at 6- and 12-month follow-up.

\section{Measures}

In this study, we used the assessments of the RCT performed at baseline and at post-treatment 3 months after baseline (directly after the intervention). The process variables were used as possible mediators. All other measures functioned as possible moderators/predictors of change and were assessed at baseline, prior to randomisation.

As outcome measures the Dutch version of the Center of Epidemiological Studies - Depression Scale was used. The CES-D (20 items, score o-60) measures symptoms of depression in the general population. Respondents rated to what extent they experienced depressive symptoms in the previous week. Higher scores mean more depressive symptoms (Haringsma et al., 2004; Radloff, 1977), and a score of 16 or higher is indicative of clinically relevant depressive symptoms (Beekman et al., 1997; Smit et al., 2006).

Measures for mediation were the Acceptance and Action Questionnaire-II (AAQ-II), measuring psychological flexibility, and the Five Facet Mindfulness QuestionnaireShort Form (FFMQ-SF), measuring mindfulness. The AAQ-II (10 items, score 10 - 70) measures the subject's willingness to be in contact with negative private events, the acceptance of these events, and the ability to live according to his/her values. Higher scores indicate more psychological flexibility (Bond et al., 2011; Fledderus et al., 2012b). The FFMQ-SF (24 items, score 24 - 120) was used to measure mindfulness in five sub-dimensions: 1 ) observing ( 4 items), defined in terms of noticing or attending to internal and external experiences; 2 ) describing ( 5 items), defined in 
terms of labelling internal experiences with words; 3 ) acting with awareness ( 5 items), defined in terms of attending to one's activities of the moment; 4) non-judging of inner experience ( 5 items), defined in terms of taking a non-evaluative stance toward thoughts and feelings; and 5) non-reactivity to inner experience ( 5 items), defined in terms of allowing thoughts and feelings to come and go, without getting caught up in or carried away by them. Facet scores range from 4 to 25 (except for observing, which ranges from 4 to 20 ), with higher scores indicating more mindfulness (Baer et al., 2006; Bohlmeijer et al., 2011a).

Measures for moderation and prediction were demographic variables, anxiety symptoms, positive mental health, and diagnostic classification. Demographic variables that were assessed as possible moderators/predictors were age, gender, and educational level. For depressive symptoms the baseline score of the CES-D was also used as a moderator/predictor. For anxiety symptoms the Hospital Anxiety and Depression Scale - Anxiety subscale (HADS-A; 7 items, score 0-21) was used, measuring symptoms of anxiety. Higher scores mean more anxiety symptoms (Spinhoven et al., 1997; Zigmond and Snaith, 1983). Positive mental health was measured with the Mental Health Continuum- Short Form (MHC-SF; 14 items, score o- 5) measuring three dimensions of positive mental health: 1) emotional well-being, defined as the presence of positive feelings/satisfaction with life; 2) social well-being, defined as positive functioning in community life; and 3) psychological well-being, defined as positive functioning in individual life. In this study the total MHC-SF score was used, with higher scores indicating greater emotional, social and psychological well-being (Keyes et al., 2008; Lamers et al., 2011). Lastly, diagnostic classification was measured with the Dutch version of the Mini-International Neuropsychiatric Interview (MINI) (Sheehan et al., 1998; van Vliet and de Beurs, 2007), supplemented with the Sheehan Disability Scale (SDS; Leon et al., 1997) to measure the severity of a disorder. Severity was defined as at least two areas of role functioning with severe role impairment due to the disorder according to the SDS. The MINI and SDS were conducted by telephone at baseline. Diagnostic classifications that were assessed as possible moderators/predictors were current disorder, current depressive disorder, and comorbidity.

\section{Analyses}

Statistical analyses were in agreement with the Intention-to-treat (ITT) principle. ITT analyses were performed using the SPSS Missing Value Analysis to impute all missing data on the continuous measures with the expectation-maximization (EM) method (Dempster et al., 1977). Available data was $96.6 \%$ at baseline and $84.5 \%$ at post-treatment. Prior to the main analyses, one-way analysis of variance (ANOVA) and chi-square tests were conducted to examine baseline differences in all potential 
moderators/predictor variables between the web-based ACT intervention and the two control conditions. Overall, significance of mediators, moderators and predictors were interpreted at $\mathrm{p}<0.05$. The statistical analyses were performed using SPSS 20.

Both simple and multiple mediation analyses were performed using PROCESS (Hayes, 2013). PROCESS is based on regression-based path-analytic framework and combines mediation and moderation into one conditional process model. PROCESS estimates the indirect effect and bias-corrected confidence intervals (CI). All analyses were based on 5000 bootstrapping samples. An indirect effect was considered significant when the $\mathrm{CI}$ did not include zero. The change score (baseline to post-treatment, baseline to 6 months, baseline to 12 months) on the outcome measure CES-D was entered as the dependent variable. The dummy variable representing treatment $(A C T=1, W L C=0$ or $E W=0$ ) and the potential mediator (measured as the change score baseline to post-treatment, baseline to 6 months, baseline to 12 months) were entered as independent variables. To control for variation in outcome score the baseline score of CES-D was entered as a covariate. Analyses were done separately for the web-based ACT intervention compared to WLC, and the webbased ACT intervention compared to the web-based EW intervention.

Linear regression models were applied for moderation variables using the PROCESS macro for SPSS. During the analyses, each potential moderator was mean centered. The change score (baseline to post-treatment, baseline to 6 months, baseline to 12 months) on the outcome measure CES-D was entered as the dependent variable. The dummy variable representing treatment, the mean centered potential moderator, and the treatment by mean centered moderator variable were entered as independent variables. To control for variation in outcome score, also in the moderator analyses, the baseline score of CES-D was entered as a covariate. Analyses were done separately for the web-based ACT intervention compared to WLC, and compared to the web-based EW intervention. In general, when there was a significant interaction effect the variable entered was interpreted as being a moderator of change.

For the predictor analyses linear regression analyses across all three groups were performed using SPSS 22, with the change score of depressive symptoms (baseline to post-treatment, baseline to 6 months, baseline to 12 months) as the dependent variable and the presumed predictors as independent variables. The outcome was adjusted for baseline values of depression by adding the baseline score of the CES-D as an additional independent variable in the regression. Also, multiple linear regressions were performed by simultaneously entering all proposed predictors as independent variables into the multiple regression. On account of the exploratory nature of the moderator and predictor analyses the borderline $\mathrm{p}$-value was not adjusted. 


\section{Results}

\section{Mediation analyses}

Table 2 shows the results of the outcomes of the simple mediation analyses. The web-based ACT intervention, as compared to the WLC, showed significantly greater improvement on the primary outcome measure post-treatment (total treatment effect $\beta=-4.73, p<0.001$ ). The bootstrap results for the indirect effects of the webbased ACT intervention on the WLC showed that changes in psychological flexibility and all mindfulness facets (except acting with awareness) significantly mediated the effect of the web-based ACT intervention on depressive symptoms post-treatment. Figure 2 shows the results of the multiple mediation model, comparing the web-based ACT intervention to the WLC. When all mediators were entered into the model, three process variables remained significant mediators of the effect on depressive symptoms: psychological flexibility (indirect effect $\beta=-1.6910,95 \%$ $C I-2.96$ to -0.76 ), and the mindfulness facets observing (indirect effect $\beta=-0.4283$, $95 \% \mathrm{CI}-1.17$ to -0.02 ), and non-judging of inner experience (indirect effect $\beta=-0.5179$, $95 \%$ CI -1.52 to -0.10). Follow-up analyses from $T_{0}$ to $T_{2}$ revealed somewhat different outcomes, with no significant mediating effect for the mindfulness facet observing (indirect effect $\beta=-0.2854,95 \% \mathrm{CI}-1.12$ to 0.40 ) and acting with awareness (indirect effect $\beta=-0.5182,95 \% C I-1.50$ to 0.10 ). Psychological flexibility (indirect effect $\beta=$ $-1.9441,95 \%$ CI -3.52 to -0.77 ), and the mindfulness facets describing (indirect effect $\beta=-1.0622,95 \% \mathrm{CI}-2.35$ to -0.17 ), non-judging of inner experience (indirect effect $\beta=$ $-1.7709,95 \%$ CI -3.32 to -0.82 ) and non-reactivity to inner experience (indirect effect $\beta=$ $-1.7766,95 \%$ CI -3.30 to -0.74 ) remained significant mediators. When all mediators were entered into the multiple mediation model, results on the follow-up analyses showed three remaining process variables: psychological flexibility (indirect effect $\beta$ $=-1.5605,95 \%$ CI -3.17 to -0.56 ) and the mindfulness facets describing (indirect effect $\beta$ $=-0.4896,95 \% \mathrm{CI}-1.63$ to -0.00 ) and non-judging of inner experience (indirect effect $\beta=$ $-0.9862,95 \%$ CI -2.23 to -0.23$)$.

The total treatment effect of the web-based ACT intervention compared to the web-based EW intervention showed a significantly greater improvement on the primary outcome measure post-treatment $(\beta=-2.70, p=0.030)$. When the indirect effects of the web-based ACT intervention were compared with the web-based EW intervention, only change in the mindfulness facet non-reactivity to inner experience appeared as mediator of the effect on depressive symptoms. When all mediators were entered in the multiple mediation model no significant mediators remained. In the follow-up analyses the mindfulness facet non-reactivity to inner experience remained a significant mediator in the simple mediation model $\left(T_{0}\right.$ to $T_{2}$, indirect effect $\beta=-0.9792,95 \% \mathrm{CI}-2.24$ to $-0.20 ; \mathrm{T}_{0}$ to $\mathrm{T}_{3}$, indirect effect $\beta=-0.8149,95 \% \mathrm{CI}$ 
-1.92 to -0.09). When all mediators were entered in the multiple mediation model, only from $T_{0}$ to $T_{1}$ the mindfulness facet non-reactivity to inner experience remained a significant mediator (indirect effect $\beta=-0.5194,95 \%-1.54$ to -0.00). No significant mediators remained for $\mathrm{T}_{0}$ to $\mathrm{T}_{2}$ and $\mathrm{T}_{0}$ to $\mathrm{T}_{3}$.

Table 2. Outcomes of simple mediation analyses assessing indirect effects of the ACT intervention through process variables on the outcome measure CES-D compared to both control conditions ${ }^{a}$.

\begin{tabular}{|c|c|c|c|c|c|c|}
\hline & \multirow[b]{2}{*}{ c'-path } & \multirow[b]{2}{*}{ a-path } & \multirow[b]{2}{*}{ b-path } & \multicolumn{3}{|c|}{$\begin{array}{l}\text { Bootstrap results for indirect } \\
\text { effects }(95 \% \mathrm{CI})\end{array}$} \\
\hline & & & & $a b$ & Lower & Upper \\
\hline \multicolumn{7}{|l|}{ ACT vs WLC } \\
\hline Psychological flexibility & $-2.81^{*}$ & $4 \cdot 39^{\star \star \star}$ & $-0.44^{\star \star \star *}$ & $-1.9220^{*}$ & -3.22 & -1.01 \\
\hline FFMQ-observing & $-4.18^{\star \star \star}$ & $0.76^{\star}$ & $-0.73^{\star \star}$ & $-0.5584^{*}$ & -1.37 & -0.09 \\
\hline FFMQ-describing & $-4.16^{\star \star \star}$ & $1.41^{\star \star}$ & -0.41 & $-0.5740^{*}$ & -1.56 & -0.04 \\
\hline FFMQ - acting with awareness & $-4.73^{\star \star *}$ & 0.05 & -0.14 & -0.0064 & -0.34 & 0.16 \\
\hline $\begin{array}{l}\text { FFMQ - non-judging of } \\
\text { inner experience }\end{array}$ & $-3.89^{\star \star}$ & $1.43^{*}$ & $-0.59^{\star \star \star}$ & $-0.8483^{*}$ & -2.06 & -0.04 \\
\hline $\begin{array}{l}\text { FFMQ - non-reactivity to } \\
\text { inner experience }\end{array}$ & $-3.41^{\star \star}$ & $2.58^{\star \star \star}$ & $-0.51^{\star \star}$ & $-1.3224^{\star}$ & -2.54 & -0.53 \\
\hline \multicolumn{7}{|l|}{ ACT vs EW } \\
\hline Psychological flexibility & -2.02 & 1.89 & $-0.36^{\star \star \star}$ & -0.6816 & -1.50 & 0.09 \\
\hline FFMQ-observing & $-2.72^{\star}$ & -0.08 & -0.24 & 0.0184 & -0.14 & 0.40 \\
\hline FFMQ-describing & $-2.52^{*}$ & 0.52 & -0.36 & -0.1872 & -0.89 & 0.07 \\
\hline FFMQ - acting with awareness & $-2.70^{\star}$ & -0.53 & 0.00 & -0.0005 & -0.00 & 0.33 \\
\hline $\begin{array}{l}\text { FFMQ - non-judging of } \\
\text { inner experience }\end{array}$ & -1.92 & 0.94 & $-0.83^{\star \star \star}$ & -0.7794 & -1.91 & 0.02 \\
\hline $\begin{array}{l}\text { FFMQ - non-reactivity to } \\
\text { inner experience }\end{array}$ & -1.72 & $1.37^{\star \star}$ & $-0.72^{\star \star \star}$ & $-0.9876^{*}$ & -2.12 & -0.31 \\
\hline
\end{tabular}

Note. $\mathrm{ACT}=$ Acceptance and Commitment Therapy; WLC = Waiting List Condition; $\mathrm{EW}=$ Expressive Writing; CES-D = Center for Epidemiological Studies - Depression Scale; FFMQ = Five Facet Mindfulness Questionnaire.

${ }^{a}$ Values are unstandardised Betas.

* Significant at $\mathrm{p}<0.05, * *$ Significant at $\mathrm{p}<0.01, * * *$ Significant at $\mathrm{p}<0.001$. 


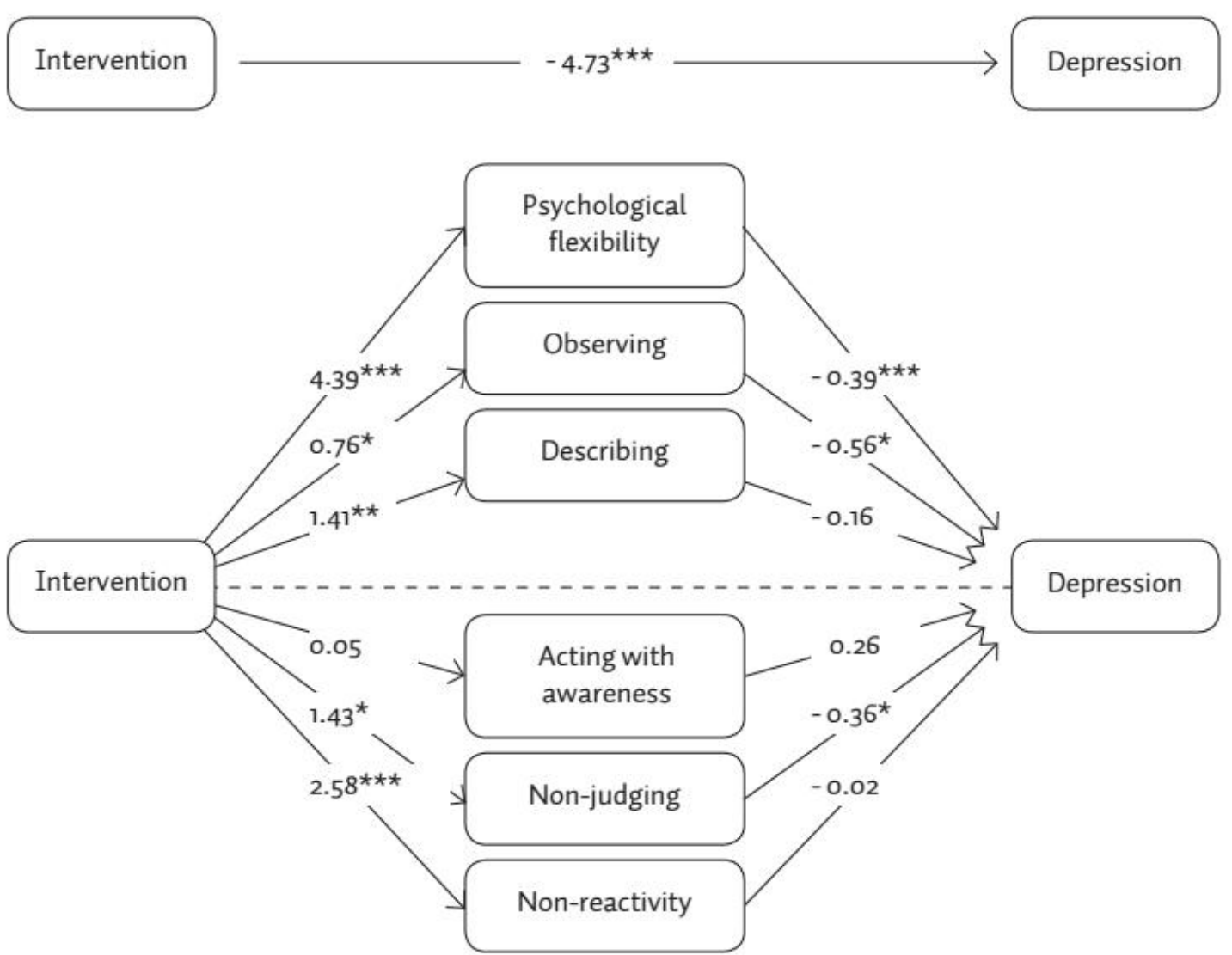

Figure 2. Multiple mediation of psychological flexibility and mindfulness as mediators of the ACT intervention compared to the WLC.

Note. Describe, describing; Observe, observing; Act aware, acting with awareness; Non-judging, non-judging of inner experience; Non-reactivity, non-reactivity to inner experience.

${ }^{\star} \mathrm{p}<.05,{ }^{\star \star} \mathrm{p}<.01,{ }^{\star \star *} \mathrm{p}<.001$.

\section{Moderation and predictor analyses}

Table 3 shows the results of the moderator analyses for the web-based ACT intervention compared to the WLC and the web-based EW intervention respectively from baseline to post-treatment. Compared to both control conditions no single significant moderator emerged, indicating that there were no significant differences for demographic characteristics, psychological measures, diagnostic classification or comorbidity. Follow-up analyses showed similar results, except for current disorder at $\mathrm{T}_{\mathrm{o}}$ when comparing the web-based $\mathrm{ACT}$ intervention versus the web-based $\mathrm{EW}$ intervention from baseline to 12 months $\left(T_{0}\right.$ to $T_{3}$ ). Participants in the web-based ACT intervention having a current disorder at $\mathrm{T}_{0}$ had more change in depressive symptoms from baseline to 12 months, when compared to the web-based EW intervention. No other significant moderators were found from $T_{0}$ to $T_{2}$ or from $T_{0}$ to $T_{3}$, for both $A C T$ versus $W L C$ and $A C T$ versus $E W$. 
Table 3- Results of moderator analyses of the ACT intervention on the outcome measure CES-D post-treatment compared to both control conditions ${ }^{a}$.

\begin{tabular}{|c|c|c|c|c|c|c|c|c|}
\hline & \multicolumn{4}{|c|}{ ACT vs WLC } & \multicolumn{4}{|c|}{ ACT vs EW } \\
\hline & \multirow[b]{2}{*}{ b } & \multicolumn{2}{|c|}{$95 \% \mathrm{CI}$} & \multirow[b]{2}{*}{$\mathbf{p}$} & \multirow[b]{2}{*}{ b } & \multicolumn{2}{|c|}{$95 \% \mathrm{CI}$} & \multirow[b]{2}{*}{$\mathbf{p}$} \\
\hline & & lower & upper & & & lower & upper & \\
\hline \multicolumn{9}{|l|}{ Demographic characteristics } \\
\hline Age & -0.101 & -0.32 & 0.12 & 0.366 & -0.026 & -0.24 & 0.19 & 0.810 \\
\hline Gender & -1.253 & -10.29 & 7.78 & 0.785 & -0.375 & -9.20 & 8.45 & 0.933 \\
\hline Educational level & 1.190 & -0.70 & 3.08 & 0.215 & 0.429 & -1.55 & 2.40 & 0.668 \\
\hline \multicolumn{9}{|l|}{ Psychological measures } \\
\hline Depression (CES-D) & -0.228 & -0.53 & 0.07 & 0.133 & -0.118 & -0.39 & 0.15 & 0.393 \\
\hline Anxiety (HADS-A) & -0.675 & -1.40 & 0.05 & 0.068 & -0.267 & -1.03 & 0.50 & 0.492 \\
\hline Emotional well-being (MHC) & 0.223 & -2.08 & 2.53 & 0.849 & -1.014 & -3.71 & 1.68 & 0.459 \\
\hline Psychological well-being (MHC) & 0.556 & -1.91 & 3.02 & 0.656 & -0.589 & -3.21 & 2.04 & 0.658 \\
\hline Social well-being (MHC) & 0.325 & -1.99 & 2.64 & 0.782 & 0.767 & -1.69 & 3.23 & 0.539 \\
\hline \multicolumn{9}{|l|}{ Diagnostic classification } \\
\hline Current disorder & 1.640 & -3.47 & 6.75 & 0.527 & -1.332 & -6.54 & 3.88 & 0.614 \\
\hline Current depressive disorder & 1.294 & -3.99 & 6.58 & 0.629 & 0.767 & $-4 \cdot 39$ & 5.93 & 0.769 \\
\hline Comorbidity & 0.775 & -5.49 & 7.04 & 0.807 & -1.195 & -7.08 & 4.69 & 0.689 \\
\hline
\end{tabular}

Note. $\mathrm{ACT}=$ Acceptance and Commitment Therapy; WLC = Waiting List Condition; EW = Expressive Writing; CES-D = Center for Epidemiological Studies - Depression Scale; HADS-A = Hamilton Anxiety and Depression Scale - Anxiety subscale; MHC = Mental Health Continuum.

${ }^{a}$ Values are unstandardized Betas.

Table 4 shows the variables that were statistically significant predictors of change in depressive symptoms over time across all conditions. Participants reporting more depressive symptoms and positive mental health (emotional, social and psychological well-being) at baseline had greater change in depressive symptoms from pre- to post-treatment, adjusted for baseline depressive symptoms. However, participants with more anxiety symptoms had less change in depressive symptoms post-treatment, adjusted for baseline depressive symptoms. No predictor effects were found for age, gender, level of education, or diagnostic classification. When all proposed predictors were entered into the multivariate regression model only baseline depressive and anxiety symptoms and emotional well-being remained significant predictors. Follow-up results on the predictor analyses showed similar results on 
the univariate analyses from $T_{0}$ to $T_{2}$. Follow-up results from $T_{0}$ to $T_{3}$ showed lack of significant predictor effect for baseline anxiety, supplemented with an additional significant predictor of level of education from pre- to 12 months. This indicates that participants reporting a higher level of education at baseline, more depressive symptoms and positive mental health (emotional, social and psychological wellbeing) at baseline experienced a greater change in depressive symptoms from pre- to 12 months, adjusted for baseline depressive symptoms. When all proposed predictors were entered into the multivariate model somewhat different results appeared. At 6 months follow-up baseline depressive and anxiety symptoms and psychological well-being remained significant predictors of change in depressive symptoms, and at 12 months follow-up only baseline depressive symptoms and psychological wellbeing remained a significant predictor of change in depressive symptoms.

Table 4 - Results of predictor analyses on the outcome measure CES-D over time collapsing across all conditions ${ }^{a}$.

\begin{tabular}{|c|c|c|c|c|c|c|}
\hline & \multicolumn{3}{|c|}{ univariate } & \multicolumn{3}{|c|}{ multivariate } \\
\hline & $T_{0}-T_{1}$ & $T_{0}-T_{2}$ & $T_{0}-T_{3}$ & $T_{0}-T_{1}$ & $T_{0}-T_{2}$ & $T_{0}-T_{3}$ \\
\hline \multicolumn{7}{|l|}{ Demographic characteristics } \\
\hline Age & 0.004 & 0.085 & 0.014 & 0.017 & 0.059 & -0.012 \\
\hline Gender & -1.806 & -2.407 & -1.394 & -1.314 & -1.689 & -0.562 \\
\hline Educational level & -0.627 & -0.781 & $-1.230^{*}$ & -0.330 & -0.498 & -0.908 \\
\hline \multicolumn{7}{|l|}{ Psychological measures } \\
\hline Depression (CES-D) & $-0.619^{\star \star *}$ & $-0.628^{\star \star *}$ & $-0.594^{\star \star \star}$ & $-0.835^{\star \star *}$ & $-0.816^{\star \star \star *}$ & $-0.804^{\star \star *}$ \\
\hline Anxiety (HADS-A) & $0.825^{\star * *}$ & $0.784^{\star \star}$ & 0.398 & $0.662^{\star \star *}$ & $0.733^{\star \star}$ & 0.374 \\
\hline $\begin{array}{l}\text { Emotional well-being } \\
(\mathrm{MHC})\end{array}$ & $-2.993^{\star \star \star}$ & $-2.833^{\star \star \star}$ & $-2.626^{\star \star}$ & $-1.711^{*}$ & -1.018 & -0.590 \\
\hline $\begin{array}{l}\text { Psychological well-being } \\
(\mathrm{MHC})\end{array}$ & $-3.153^{\star * *}$ & $-3.549^{* * *}$ & $-4.089^{\star \star \star}$ & -1.406 & $-2.074^{\star}$ & $-3.356^{\star *}$ \\
\hline Social well-being (MHC) & $-2.109^{\star \star \star}$ & $-2.170^{\star \star}$ & $-2.582^{\star \star}$ & -0.514 & -0.628 & -0.359 \\
\hline \multicolumn{7}{|l|}{ Diagnostic classification } \\
\hline Current disorder & 0.415 & -0.678 & 0.271 & -0.093 & -0.620 & -0.122 \\
\hline Current depressive disorder & 0.004 & -0.684 & 1.201 & -1.578 & -1.158 & 1.264 \\
\hline Comorbidity & 1.447 & -0.755 & -0.668 & 0.451 & -1.628 & -2.160 \\
\hline
\end{tabular}

Note. CES-D, Center for Epidemiological Studies - Depression Scale; HADS-A, Hamilton Anxiety and Depression Scale - Anxiety subscale; MHC, Mental Health Continuum.

a Outcome adjusted for baseline value of depression; Values are unstandardized Betas.

${ }^{\star} \mathrm{p}<0.05,{ }^{\star *} \mathrm{p}<0.01,{ }^{* \star *} \mathrm{p}<0.001$. 


\section{Discussion}

The aim of the present study was to examine why and how a web-based ACT intervention for depressive symptoms is effective, and to identify for whom and under what circumstances this intervention has differential effects. Psychological flexibility and two mindfulness facets were the strongest mediators of the treatment effect of the ACT intervention versus the WLC. Only change in the mindfulness facet non-reactivity to inner experience appeared as mediator of the treatment effect of the ACT intervention versus the web-based EW intervention. The moderator analyses demonstrated that the effects of the web-based ACT intervention on depressive symptoms did not vary according to baseline patient characteristics when compared to both control groups. However, baseline symptoms and positive mental health were identified as predictors of outcome when collapsing across all conditions. Follow-up results on mediation and moderation/prediction were somewhat similar.

The findings of this study on mediation corroborate the evidence that psychological flexibility and mindfulness are distinct process mechanisms that mediate the effect of web-based ACT intervention on depressive symptoms. This confirms the relevance of psychological flexibility as the central therapeutic mechanism in the ACT model and further strengthens the theoretical framework of ACT (Hayes et al., 2012). Furthermore, our results give empirical support for mindfulness as an important mediator in the ACT model. Both the processes of psychological flexibility and mindfulness can be considered within a broader context of emotion regulation as a transdiagnostic mechanism of change in mindfulness- and acceptance based interventions (e.g. Chiesa et al., 2013; Farb et al., 2012; Hölzel et al., 2011). Chiesa et al. (2014) suggest that such interventions may in general enhance positive emotional regulation strategies, as well as self-compassion levels, and decrease rumination and experiential avoidance. They specifically suggested that short-term mindfulness meditation practitioners might achieve these benefits by means of a top-down regulation of the prefrontal areas on limbic areas (Chiesa et al., 2013). Hence, the studies on the broader context of emotion regulation endorse mindfulness and psychological flexibility processes as an emotion regulation process within the theoretical framework of ACT.

Mediation analyses of ACT versus EW showed that no mediators remained significant in the follow-up analyses of the multiple mediation, compared to the simple mediation. In the previously reported results from the RCT (Pots et al., 2015), we found that the web-based ACT intervention had similar effects on depressive symptoms and secondary measures after 6 and 12 months compared to the EW intervention. Additionally, the web-based EW condition showed significant improvement compared with the WLC from baseline to follow-up ( $T_{0}$ to $\left.T_{2}\right)$ for psychological 
flexibility and some facets of mindfulness, and no significant improvement on other outcome measures. The results of the current study show that there is no unique mediator of change when comparing the web-based ACT intervention versus the web-based EW intervention. These findings, combined with the results from the $R C T$, suggest that the web-based EW intervention shares important therapeutic mechanisms with the web-based ACT intervention, notably psychological flexibility and facets of mindfulness. This lack of specificity is not an isolated finding. A recent meta-analysis of Goyal et al. (2014) found that studies comparing a mindfulness intervention with comparison conditions (active controls) showed no significant differences on the FFMQ or other mindfulness measures. Goldberg et al. (2015) compared a Mindfulness-based Stress Reduction program with an active control condition that did not include the instruction in mindfulness meditation, and a waiting list control. In accordance with our results their active control condition also showed an improvement over time on the FFMQ, suggesting that their active control condition did induce mindfulness although it was not specifically trained. The literature on the processes of change in EW is however still in its infancy. Some researchers have indicated that acceptance or experiential disclosure is an important mechanism (Frattaroli, 2006; Pennebaker, 1997). The results of this study confirm the concept that acceptance is a process mechanism of EW as both psychological flexibility and mindfulness encompass acceptance, but future studies are necessary to shed more light on the therapeutic mechanisms in EW. This might, however, prove difficult in lack of a solid theoretical model underlying EW.

A second aim of this study was to explore moderators and predictors of change to identify characteristics of those likely to benefit. Since none of the potential moderators were significant for ACT versus WLC and these effects were maintained in the follow-up analyses, the results indicate that there are no restrictions to the allocation of the web-based ACT intervention and that the web-based ACT intervention can work for different subpopulations. This is a significant finding, as web-based interventions can have a large incremental impact in availability and accessibility of interventions (Andersson and Titov, 2014; Andrews et al., 2010; Barak et al., 2008). To attain this impact, however, it is highly necessary for web-based interventions to ensure treatment adherence in addition to treatment effectiveness. The high adherence in this study might indicate feasibility and compatibility with the users by using a holistic approach based on persuasive technology, such as the usage of SMS support, multi-media, feedback and tailoring, which has been shown to be positively related to adherence (Kelders et al., 2012). This might indicate that the persuasive technology used in the web-based ACT intervention made the intervention more suitable to individual's needs. When comparing the web-based ACT intervention versus the web-based EW intervention similar results were found, except for a single 
moderator at 12 months follow-up, notably having a current disorder at baseline. This result is interesting as it indicates that having a current disorder at baseline is predictive of more improvement in depressive symptoms at 12 months follow-up, when receiving ACT versus EW. No other moderators were found for ACT versus EW. In short, the results suggest that the level of symptoms is more severe ACT seems to be more beneficial at long term follow-up, compared to EW. In addition to moderator analyses the predictor analyses indicated that participants reporting more depressive symptoms, and emotional, psychological and social well-being at baseline, had greater change in depressive symptoms from pre- to post-treatment. However, having more anxiety symptoms at baseline seemed to lead to less change in depressive symptoms from pre-to post-treatment. Follow-up results showed similar results on the univariate analyses. In the multivariate model baseline depressive and anxiety symptoms, and psychological well-being remained significant predictors of change in depressive symptoms at 6 months follow-up, and only baseline depressive symptoms and psychological well-being remained a significant predictor of change in depressive symptoms at 12 months follow-up. These results indicate that people with more depressive symptoms and positive psychological functioning have the best opportunities for improvement. This latter finding is in accordance with the finding that positive mental health is a protective factor against mental illness (Keyes et al., 2010; Wood and Joseph, 2010).

Previous studies in different populations on moderators and predictors of face-toface ACT interventions are sparse and non-conclusive on what moderators and predictors are specific to help tailor interventions to different subpopulations (Davies et al., 2015; Flaxman and Bond, 2010; Fledderus et al.; 2012a, Niles et al.; 2014, Turner et al., 2007; Wolitzky-Taylor et al., 2012). Some studies found that greater baseline symptoms, neuroticism, and experiential avoidance are moderators or predictors, but the results are not consistent (e.g. Fledderus et al., 2012a; Niles et al., 2014; Turner et al., 2007; Wolitzky-Taylor et al., 2012). A recent meta-analysis on 49 randomised controlled trails comparing computerised CBT (CCBT) to other therapies and waiting list controls, sheds some light on moderators of web-based interventions (Grist and Cavanagh, 2013). The findings indicated that the benefit of CCBT decreases as age increases. Also type of control group moderated effect sizes, with higher effect sizes for comparisons of CCBT with inactive controls. Interestingly, no further significant moderating effect were found for any of the variables (demographic characteristics, severity of symptoms, type of intervention, type of support). A highly prevalent and important potential moderator is comorbidity. In this study, comorbidity was not found to be a moderator or predictor of effects, which is of importance because the majority of web-based interventions have targeted specific disorders. Interestingly, Wolitzky et al. (2012) found that ACT outperformed Cognitive Behaviour Therapy (CBT) among 
those with comorbid mood disorders. In contrast, Niles et al. (2014) also compared CBT with ACT and found no such effect. Despite the importance of these findings on face-to-face interventions, it is to be expected that results of face-to-face ACT interventions are not comparable to web-based ACT interventions as these latter are expected to place a greater burden on self-efficacy or resilience. The fact that in the current study there were no moderators found when comparing ACT versus WLC, suggests that web-based ACT can work for different subpopulations. As the analyses were exploratory, the results should be interpreted with caution. Overall, we can conclude that the studies so far that have included moderator and predictor analyses in web-based ACT interventions are sparse and mostly exploratory, highlighting the necessity of research in this area. Future research should include more formal moderator and predictor analyses to be able to better tailor individuals to treatment.

This study has several limitations. First, the RCT was not powered for the moderator analyses. Since the analyses were exploratory and post-hoc, the absence of significant moderators could well be a result of inadequate statistical power (Simon and Perlis, 2010). However, our study is in accordance with the methodological requirements of exploratory moderator analyses (Pincus et al., 2011). Second, all mediation analyses were performed using baseline to follow-up measurements of both processes and outcomes, precluding an evaluation of temporal precedence. Future studies could use more sophisticated designs to address temporal precedence of the process variables, such as a cross-lagged panel design (Fledderus et al., 2013). Third, the original sample in the RCT was predominantly female with a rather high level of education, recruited from the general Dutch community through newspapers. This restricts the generalisability of the results to gender and education. The fact that our sample was fairly homogeneous could also be an argument for the fact that no moderators were found (Flaxman and Bond, 2010). Fourth, in this study we focused on the overarching processes of the theoretical model ACT, psychological flexibility and mindfulness. Nowadays, more specific measures of the individual core processes of ACT such as the Engaged Living Scale (Trompetter et al., 2013) measuring an engaged response style, are available.

\section{Conclusions}

Overall, this study was the first to assess mediators, moderators and predictors of change in a web-based ACT treatment for depressive symptoms. The findings demonstrate that web-based ACT is successful in enhancing psychological flexibility and facets of mindfulness and that these changes mediate the short and long-term effects on depressive symptoms. The findings also demonstrate that at present there is no 
reason to exclude people from web-based ACT, though when the level of depressive symptoms is more severe web-based ACT seems to be more beneficial at long term follow-up, compared to web-based EW. Furthermore, higher levels of depressive symptoms and higher levels of psychological well-being predict better long term outcomes. 


\section{References}

A-Tjak, J. G. L., Davis, M. L., Morina, N., Powers, M. B., Smits, J. A. J. \& Emmelkamp, P. M. G. (2015). A meta-analysis of the efficacy of acceptance and commitment therapy for clinically relevant mental and physical health problems. Psychotherapy and Psychosomatics 84, 30-36.

Aldao, A. \& Nolen-Hoeksema, S. (2010). Specificity of cognitive emotion regulation strategies: A transdiagnostic examination. Behaviour Research and Therapy 48, 974-983.

Andersson, G. \& Titov, N. (2014). Advantages and limitations of Internet-based interventions for common mental disorders. World Psychiatry 13, 4-11.

Andrews, G., Cuijpers, P., Craske, M. G., McEvoy, P. \& Titov, N. (2010). Computer therapy for the anxiety and depressive disorders is effective, acceptable and practical health care: A meta-analysis. PLoS ONE 5, el3196.

Baer, R. A., Smith, G. T., Hopkins, J., Krietemeyer, J. \& Toney, L. (2006). Using self-report assessment methods to explore facets of mindfulness. Assessment 13, 27-45.

Barak, A., Hen, L., Boniel-Nissim, M. \& Shapira, N. (2008). A comprehensive review and a metaanalysis of the effectiveness of internet-based psychotherapeutic interventions. Journal of Technology in Human Services 26, 109-160.

Beekman, A. T. F., Deeg, D. J. H., Van Limbeek, J., Braam, A. W., De Vries, M. Z. \& Van Tilburg, W. (1997). Criterion validity of the Center for Epidemiologic Studies Depression scale (CES-D): Results from a community-based sample of older subjects in the Netherlands. Psychological Medicine 27, 231-235.

Bohlmeijer, E. \& Hulsbergen, M. (2013). A beginner's guide to mindfulness: live in the moment. Open University Press: Oxford.

Bohlmeijer, E., Ten Klooster, P. M., Fledderus, M., Veehof, M. \& Baer, R. (2011a). Psychometric properties of the five facet mindfulness questionnaire in depressed adults and development of a short form. Assessment 18, 308-320.

Bohlmeijer, E. T., Fledderus, M., Rokx, T. A. J. J. \& Pieterse, M. E. (2011b). Efficacy of an early intervention based on acceptance and commitment therapy for adults with depressive symptomatology: Evaluation in a randomized controlled trial. Behaviour Research and Therapy 49, 62-67.

Bond, F. W., Hayes, S. C., Baer, R. A., Carpenter, K. M., Guenole, N., Orcutt, H. K., ... Zettle, R. D. (2011). Preliminary Psychometric Properties of the Acceptance and Action Questionnaire-II: A Revised Measure of Psychological Inflexibility and Experiential Avoidance. Behavior Therapy $42,676-688$.

Bricker, J., Wyszynski, C., Comstock, B. \& Heffner, J. L. (2013). Pilot randomized controlled trial of web-based acceptance and commitment therapy for smoking cessation. Nicotine and Tobacco Research 15, 1756-1764.

Buhrman, M., Skoglund, A., Husell, J., Bergström, K., Gordh, T., Hursti, T., ... Andersson, G. (2013). Guided internet-delivered acceptance and commitment therapy for chronic pain patients: A randomized controlled trial. Behaviour Research and Therapy 51, 307-315.

Chiesa, A., Anselmi, R. \& Serretti, A. (2014). Psychological mechanisms of mindfulness-based interventions: What do we know? Holistic Nursing Practice 28, 124-148.

Chiesa, A., Serretti, A. \& Jakobsen, J. C. (2013). Mindfulness: Top-down or bottom-up emotion regulation strategy? Clinical Psychology Review 33, 82-96.

Christensen, H., Griffiths, K. M. \& Farrer, L. (2009). Adherence in internet interventions for anxiety and depression. Journal of Medical Internet Research 11, e13.

Davies, C. D., Niles, A. N., Pittig, A., Arch, J. J. \& Craske, M. G. (2015). Physiological and behavioral indices of emotion dysregulation as predictors of outcome from cognitive behavioral therapy and acceptance and commitment therapy for anxiety. Journal of Behavior Therapy and Experimental Psychiatry 46, 35-43.

Dempster, A. P., Laird, N. M. \& Rubin, D. B. (1977). Maximum likelihood from incomplete data via the EM algorithm. Journal of the Royal Statistical Society Series B Methodological 39, 1-38. 
Eysenbach, G. (2005). The law of attrition. Journal of Medical Internet Research 7, en1.

Farb, N. A. S., Anderson, A. K. \& Segal, Z. V. (2012). The mindful brain and emotion regulation in mood disorders. Canadian Journal of Psychiatry 57, 70-77.

Flaxman, P. E. \& Bond, F. W. (2010). Worksite stress management training: Moderated effects and clinical significance. Journal of Occupational Health Psychology 15, 347-358.

Fledderus, M., Bohlmeijer, E. T., Fox, J. P., Schreurs, K. M. G. \& Spinhoven, P. (2013). The role of psychological flexibility in a self-help acceptance and commitment therapy intervention for psychological distress in a randomized controlled trial. Behaviour Research and Therapy 51, 142-151.

Fledderus, M., Bohlmeijer, E. T., Pieterse, M. E. \& Schreurs, K. M. G. (2012a). Acceptance and commitment therapy as guided self-help for psychological distress and positive mental health: A randomized controlled trial. Psychological Medicine 42, 485-495.

Fledderus, M., Bohlmeijer, E. T., Smit, F. \& Westerhof, G. J. (2010). Mental health promotion as a new goal in public mental health care: A randomized controlled trial of an intervention enhancing psychological flexibility. American Journal of Public Health 100, 2372-2378.

Fledderus, M., Oude Voshaar, M. A. H., Ten Klooster, P. M. \& Bohlmeijer, E. T. (2012b). Further evaluation of the psychometric properties of the acceptance and action questionnaire-II. Psychological Assessment 24, 925-936.

Forman, E. M., Chapman, J. E., Herbert, J. D., Goetter, E. M., Yuen, E. K. \& Moitra, E. (2012). Using Session-by-Session Measurement to Compare Mechanisms of Action for Acceptance and Commitment Therapy and Cognitive Therapy. Behavior Therapy 43, 341-354.

Frattaroli, J. (2006). Experimental disclosure and its moderators: A meta-analysis. Psychological Bulletin 132, 823-865.

Goldberg, S. B., Wielgosz, J., Dahl, C., Schuyler, B., MacCoon, D. S., Rosenkranz, M., ... Davidson, R. J. (2015). Does the Five Facet Mindfulness Questionnaire Measure What We Think It Does? Construct Validity Evidence From an Active Controlled Randomized Clinical Trial. Psychological Assessment, article in press.

Goyal, M., Singh, S., Sibinga, E. M. S., Gould, N. F., Rowland-Seymour, A., Sharma, R., ... Haythornthwaite, J. A. (2014). Meditation programs for psychological stress and well-being: A systematic review and meta-analysis. JAMA Internal Medicine 174, 357-368.

Grist, R. \& Cavanagh, K. (2013). Computerised cognitive behavioural therapy for common mental health disorders, what works, for whom under what circumstances? A systematic review and meta-analysis. Journal of Contemporary Psychotherapy 43, 243-251.

Haringsma, R., Engels, G. I., Beekman, A. T. F. \& Spinhoven, P. (2004). The criterion validity of the Center for Epidemiological Studies Depression Scale (CES-D) in a sample of self-referred elders with depressive symptomatology. International Journal of Geriatric Psychiatry 19, 558-563.

Hayes, A. (2013). Introduction to mediation, moderation and conditional process analysis. Guilford Press: New York.

Hayes, S. C., Luoma, J. B., Bond, F. W., Masuda, A. \& Lillis, J. (2006). Acceptance and Commitment Therapy: Model, processes and outcomes. Behaviour Research and Therapy 44, 1-25.

Hayes, S. C., Strosahl, K. D. \& Wilson, K. G. (2012). Acceptance and Commitment Therapy: the Process and Practice of Mindful Change. Guilford Press: New York.

Hesser, H., Gustafsson, T., Lundén, C., Henrikson, O., Fattahi, K., Johnsson, E., ... Andersson, G. (2012). A randomized controlled trial of internet-delivered cognitive behavior therapy and acceptance and commitment therapy in the treatment of tinnitus. Journal of Consulting and Clinical Psychology 80, 649-661.

Hölzel, B. K., Lazar, S. W., Gard, T., Schuman-Olivier, Z., Vago, D. R. \& Ott, U. (2011). How does mindfulness meditation work? Proposing mechanisms of action from a conceptual and neural perspective. Perspectives on Psychological Science 6, 537-559.

Kabat-Zinn, J. (1990). Full catastrophe living: Using the wisdom of your body and mind to face stress, pain and illness. Delacorte: New York.

Kashdan, T. B. \& Rottenberg, J. (2010). Psychological flexibility as a fundamental aspect of health. Clinical Psychology Review 30, 865-878. 
Kazdin, A. E. (2007). Mediators and mechanisms of change in psychotherapy research. Annual Review of Clinical Psychology 3, 1-27.

Kelders, S. M., Kok, R. N., Ossebaard, H. C. \& Van Gemert-Pijnen, J. E. W. C. (2012). Persuasive system design does matter: A systematic review of adherence to web-based interventions. Journal of Medical Internet Research 14, e152.

Kelders, S. M., Pots, W. T., Oskam, M. J., Bohlmeijer, E. T. \& Van Gemert-Pijnen, J. E. (2013). Development of a web-based intervention for the indicated prevention of depression. BMC Medical Informatics and Decision Making 13, 1-11.

Keyes, C. L. M., Dhingra, S. S. \& Simoes, E. J. (2010). Change in level of positive mental health as a predictor of future risk of mental Illness. American Journal of Public Health 100, 2366-2371.

Keyes, C. L. M., Wissing, M., Potgieter, J. P., Temane, M., Kruger, A. \& van Rooy, S. (2008). Evaluation of the mental health continuum-short form (MHC-SF) in Setswana-speaking South Africans. Clinical Psychology and Psychotherapy 15, 181-192.

Kocovski, N. L., Fleming, J. E., Hawley, L. L., Huta, V. \& Antony, M. M. (2013). Mindfulness and acceptance-based group therapy versus traditional cognitive behavioral group therapy for social anxiety disorder: A randomized controlled trial. Behaviour Research and Therapy 51, 889-898.

Kraemer, H. C., Wilson, G. T., Fairburn, C. G. \& Agras, W. S. (2002). Mediators and moderators of treatment effects in randomized clinical trials. Archives of General Psychiatry 59, 877-883.

Lamers, S. M., Westerhof, G. J., Bohlmeijer, E. T., Ten Klooster, P. M. \& Keyes, C. L. (2011). Evaluating the psychometric properties of the mental health Continuum-Short Form (MHC-SF). Journal of Clinical Psychology 67, 99-110.

Lappalainen, P., Granlund, A., Siltanen, S., Ahonen, S., Vitikainen, M., Tolvanen, A. \& Lappalainen, R. (2014). ACT Internet-based vs face-to-face? A randomized controlled trial of two ways to deliver Acceptance and Commitment Therapy for depressive symptoms: An 18-month follow-up. Behaviour Research and Therapy 61, 43-54.

Leon, A. C., Olfson, M., Portera, L., Farber, L. \& Sheehan, D. V. (1997). Assessing psychiatric impairment in primary care with the Sheehan Disability Scale. International Journal of Psychiatry in Medicine 27, 93-105.

MacKinnon, D. P. (2011). Integrating mediators and moderators in research design. Research on Social Work Practice 21, 675-681.

McCracken, L. M. \& Gutiérrez-Martínez, O. (2011). Processes of change in psychological flexibility in an interdisciplinary group-based treatment for chronic pain based on Acceptance and Commitment Therapy. Behaviour Research and Therapy 49, 267-274.

Niles, A. N., Burklund, L. J., Arch, J. J., Lieberman, M. D., Saxbe, D. \& Craske, M. G. (2014). Cognitive mediators of treatment for social anxiety disorder: Comparing acceptance and commitment therapy and cognitive-behavioral therapy. Behavior Therapy 45, 664-677.

Öst, L. G. (2014). The efficacy of Acceptance and Commitment Therapy: An updated systematic review and meta-analysis. Behaviour Research and Therapy 61, 105-121.

Pennebaker, J. W. (1997). Writing about emotional experiences as a therapeutic process. Psychological Science 8, 162-166.

Pincus, T., Miles, C., Froud, R., Underwood, M., Carnes, D. \& Taylor, S. J. (2011). Methodological criteria for the assessment of moderators in systematic reviews of randomised controlled trials: A consensus study. In BMC Medical Research Methodology.

Pots, W. T. M., Fledderus, M., Meulenbeek, P. A. M., Klooster ten, P. M., Schreurs, K. M. \& Bohlmeijer, E. T. (2015). Acceptance and commitment therapy as a web-based intervention for depressive symptoms: randomised controlled trial. British Journal of Psychiatry 208, 69-77.

Radloff, L. S. (1977). The CES-D scale, a self report depression scale for reseach in the general population. Applied Psychological Measurement 1, 385-401.

Ruiz, F. J. (2010). A review of acceptance and commitment therapy (ACT) empirical evidence: Correlational, experimental psychopathology, component and outcome studies. International Journal of Psychology and Psychological Therapy 10, 125-162. 
Sheehan, D. V., Lecrubier, Y., Sheehan, K. H., Amorim, P., Janavs, J., Weiller, E., ... Dubar, G. C. (1998). The Mini-International Neuropsychiatric Interview (M.I.N.I.): the development and validation of a structured diagnostic psychiatric interview for DSM-IV and ICD-10. Journal of Clinical Psychiatry 59, 22-33.

Simon, G. E. \& Perlis, R. H. (2010). Personalized medicine for depression: Can we match patients with treatments? American Journal of Psychiatry 167, 1445-1455.

Smit, F., Ederveen, A., Cuijpers, P., Deeg, D. \& Beekman, A. (2006). Opportunities for cost-effective prevention of late-life depression: An epidemiological approach. Archives of General Psychiatry 63, 290-296.

Spinhoven, P., Ormel, J., Sloekers, P. P. A., Kempen, G. I. J. M., Speckens, A. E. M. \& Van Hemert, A. M. (1997). A validation study of the hospital anxiety and depression scale (HADS) in different groups of Dutch subjects. Psychological Medicine 27, 363-370.

Trompetter, H. R., Bohlmeijer, E. T., Fox, J. P. \& Schreurs, K. M. G. (2015). Psychological flexibility and catastrophizing as associated change mechanisms during online Acceptance \& Commitment Therapy for chronic pain. Behaviour Research and Therapy 74, 50-59.

Trompetter, H. R., Bohlmeijer, E. T., Veehof, M. M. \& Schreurs, K. M. G. (2014). Internet-based guided self-help intervention for chronic pain based on Acceptance and Commitment Therapy: A randomized controlled trial. Journal of Behavioral Medicine 38, 66-80.

Trompetter, H. R., Ten Klooster, P. M., Schreurs, K. M. G., Fledderus, M., Westerhof, G. J. \& Bohlmeijer, E. T. (2013). Measuring values and committed action with the engaged living scale (ELS): Psychometric evaluation in a nonclinical sample and a chronic pain sample. Psychological Assessment 25, 1235-1246.

Turner, J. A., Holtzman, S. \& Mancl, L. (2007). Mediators, moderators, and predictors of therapeutic change in cognitive-behavioral therapy for chronic pain. Pain 127, 276-286.

Van Vliet, I. M. \& De Beurs, E. (2007). The MINI-International Neuropsychiatric Interview (M.I.N.I.). A brief structured diagnostic psychiatric interview for DSM-IV and ICD-10 psychiatric disorders [in Dutch]. Tijdschrift voor Psychiatrie 49, 393-397.

Wicksell, R. K., Olsson, G. L. \& Hayes, S. C. (2011). Mediators of change in Acceptance and Commitment Therapy for pediatric chronic pain. Pain 152, 2792-2801.

Wolitzky-Taylor, K. B., Arch, J. J., Rosenfield, D. \& Craske, M. G. (2012). Moderators and non-specific predictors of treatment outcome for anxiety disorders: A comparison of cognitive behavioral therapy to acceptance and commitment therapy. Journal of Consulting and Clinical Psychology 80, 786-799.

Wood, A. M. \& Joseph, S. (2010). The absence of positive psychological (eudemonic) well-being as a risk factor for depression: A ten year cohort study. Journal of Affective Disorders 122, 213-217.

Zigmond, A. S. \& Snaith, R. P. (1983). The hospital anxiety and depression scale. Acta Psychiatrica Scandinavica 67, 361-370. 


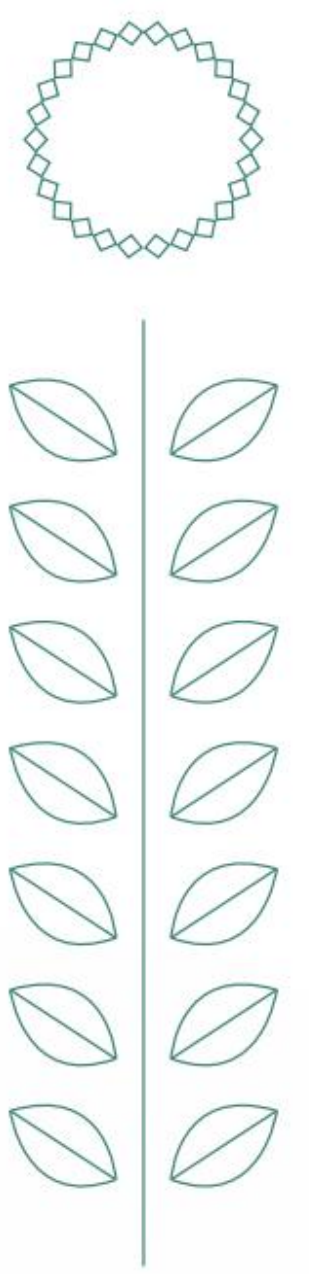

\section{CHAPTER 7}




\section{Web-based Acceptance and}

Commitment Therapy versus

Expressive Writing and waitlist in depression: economic evaluation alongside a three-armed randomised trial

Pots, W.T.M., Smit, F., Meulenbeek, P.A.M. \& Bohlmeijer, E.T. Web-based Acceptance and Commitment Therapy versus Expressive Writing and waitlist in depression: economic evaluation alongside a three-armed randomised trial. Submitted. 


\section{Abstract}

Web-based interventions are assumed to be cost-effective, but trials reporting on cost-effectiveness are scarce. This study was set out as a health-economic evaluation alongside a randomised controlled trial of a web-based Acceptance and Commitment Therapy (ACT) intervention for depressive symptoms. ACT has been demonstrated to be effective in reducing depressive symptoms. Mild to moderately depressed adults from the general population were randomised to web-based ACT ( $n=82$ ), an active control condition (web-based Expressive Writing; EW; $n=67$ ) and a waiting list control condition (WLC; $n=87$ ). Follow-up was at 6 months post baseline, with an extended follow-up for only ACT versus EW at 12 months. Treatment response was defined as a clinically significant change in depressive symptoms and quality-adjusted life years (QALYs) gained. Cost-effectiveness and cost-utility analyses showed that web-based ACT was the dominant intervention in comparison with web-based EW at 6 months. This effect recedes at 12 months, where ACT is no longer significantly more effective than EW. Compared to the WLC, the ACT intervention was not significantly more effective in increasing health gains at 6 months, and the likelihood of the web-based ACT intervention being superior was $39 \%$ and $33 \%$ for cost-effectiveness and cost-utility respectively. Sensitivity analyses showed that with realistically lower intervention costs web-based ACT was the dominant intervention compared to both control conditions. In conclusion, the web-based ACT intervention is the dominant intervention in comparison with EW at 6 months, and with lower intervention costs superior in costs compared to both EW and WLC. 


\section{Introduction}

Depression is a common disorder characterised by depressed mood or loss of interest, impairing one's personal, social and professional functioning. In The Netherlands depression is a highly prevalent disorder (De Graaf et al., 2012a), that has a large adverse impact on quality of life, yielding high economic costs (De Graaf et al., 2012b). From both a health and an economic perspective, the burden to society is significant. By the year 2030 depression's disease burden is expected to rank first in high-income countries (Mathers and Loncar, 2006). Besides evaluating the efficacy and effectiveness of psychological treatments, economic evaluations are necessary to determine the cost-effectiveness of interventions. However, the evidence-base for cost-effective treatment and prevention of depression is limited (Mihalopoulos and Chatterton, 2015), and even more so for web-based intervention (Donker et al., 2015; Hedman et al., 2012). To address this issue, we conducted cost-effectiveness and cost-utility analyses of a web-based Acceptance and Commitment Therapy (ACT) intervention alongside the randomised controlled trial (RCT). ACT is a behavioural cognitive therapy that uses acceptance and mindfulness strategies together with commitment and behaviour change strategies (Hayes et al., 2006; Hayes et al., 2012). Its efficacy is empirically supported by a series of meta-analyses, most recently A-Tjak et al. (2015), Hacker et al. (2016), Öst, (2014), and Ruiz (2010). Web-based interventions are expected to reduce the costs of health service delivery as they generally involve minimal involvement of clinicians. The primary objective of our study was to test the hypothesis that the web-based ACT intervention would lead to better health (i.e. reduction in depressive symptoms and improvement in quality of life) against lower costs.

\section{Methods}

\section{Design}

The study was designed as a pragmatic randomised trial in three parallel groups: the web-based ACT intervention ( $n=82$ ), an active control condition called the web-based Expressive Writing $(E W)$ intervention $(n=67)$, and a waiting list control condition $(W L C ; n=87)$. Measurements were taken at baseline, at 3 months follow-up (posttreatment), and at 6 months post baseline. An extended follow-up was taken for comparison of $A C T$ versus $E W$ at 12 months, since the $W L C$ received their intervention after 6 months and were excluded from further analyses. A sample size of 50 participants per condition was needed to detect an effect size of 0.40 (Cohen's d) in depressive symptom severity (primary outcome) with a statistical power of $(1-\beta)=0.80$ in a two-tailed test $(p<0.05)$. Anticipating a worst-case scenario of a $40 \%$ drop-out for 
web-based interventions, 235 people were needed for randomisation. The study was approved by an independent medical ethics committee for research in mental health settings in The Netherlands (METiGG; number NL33619.097.100) and recorded in the Dutch primary trial register for clinical trials (The Netherlands Trail Register, NTR2736). The clinical findings of this trial are reported in detail elsewhere (Pots et al., 2015).

\section{Participants and procedure}

Participants with mild to moderate depressive symptoms were recruited through advertisements in Dutch national newspapers and on the Internet in January and February 2011. To be included, participants must be 18 years or older with mild to moderate depressive symptoms ( $>10$ on the Dutch version of the Center of Epidemiological Studies Depression Scale; CES-D (Fledderus et al., 2012)). At initial screening, participants were excluded when: a) reporting too few depressive symptoms ( $\leq 10$ on the CES-D); b) receiving psychological or pharmacological treatment for mental complaints within the last three months; $c$ ) having problems reading or writing Dutch; and d) being unable to invest approximately three hours per week in the intervention and daily exercises. Furthermore, participants with severe psychopathology were excluded when diagnosed with a current severe mental disorder or when having a moderate to high suicide risk, according to the Dutch version of the Mini International Neuropsychiatric Interview (Sheehan et al., 1998; Van Vliet and De Beurs, 2007) and the Sheehan Disability Scale (Leon et al., 1997). Those meeting the exclusion criteria were advised to consult their general practitioner.

Participants were randomised after the clinical interview and baseline measurement. Randomisation was conducted centrally by an independent statistician using a computer-generated randomisation list and stratified for gender, education (low versus middle-high) and age ( $\leq 50$ versus $>50$ ). A total of 436 persons were assessed for eligibility (For flowchart, see Appendix A). Of the eligible respondents, 93 persons were excluded based on initial screening criteria conducted within the automated computerised assessment battery. A total of 343 participants met the initial screening criteria for inclusion and were contacted by phone for a structured interview. Based on the clinical interview, 58 participants were excluded, and a further 29 respondents could not be reached. Of the 256 included participants, 20 did not complete the baseline measurement. Hence a total of 236 participants were randomised.

\section{Interventions}

Patients in ACT and in the active control condition (EW) were both offered an online intervention, which could be worked through in nine to twelve weeks. The web-based ACT intervention "Living to the Full" is a self-help intervention that has shown to 
be effective as a group course and as a bibliotherapeutic intervention with e-mail support (Bohlmeijer et al., 2011; Fledderus et al., 2012; Fledderus et al., 2010). The sessions of the web-based ACT intervention are based on six core processes of ACT that together promote psychological flexibility (Hayes et al., 2012). Each session is exemplified with experiential exercises and metaphors to illustrate the ACT processes, text messages, tailored stories for motivation, and an option to personalise the homepage. Furthermore, participants were encouraged to practice daily mindfulness exercises that were provided on audio, downloadable within the web-based intervention. A comprehensive description of the development of the intervention can be found elsewhere (Kelders et al., 2013).

The active control condition was a web-based EW intervention based on Pennebaker's expressive writing paradigm (Pennebaker, 1997). A meta-analysis of Frattaroli (2006) shows that EW has small effects (unweighted $r$ - effect size $=0.075$, range $-0.291-0.592$; comparable to $d=0.151$ ) on various mental health outcomes. EW generally involves asking participants to write about a highly stressful experience (particularly their deepest thoughts and feelings), usually in 3 to 4 sessions. We extended and adapted the method of Pennebaker into an intervention format equal to the ACT intervention. Every session started with psycho-education on emotions and emotion regulation based on Gross' process model of emotion regulation (Gross and Thompson, 2011), and was followed by instructions of the method of EW. The EW intervention was also web-based and comprised of nine online sessions. In general, people were instructed to write about emotional experiences for 15 to 30 minutes on at least three days during one week. Support was provided for participants in both intervention conditions by trained graduate psychology students, by means of weekly email feedback. Counselling comprised of personal feedback on key learning points and exercises, accompanied by positive encouragement and support. The feedback was offered once a week after participants completed a session. Each counsellor spent an average of 20 minutes on each participant providing feedback and answering questions by means of email.

\section{Waitlist control condition}

Participants randomised to the waitlist condition were offered no intervention, but had unlimited access to other forms of care. Six months after baseline these participants could start a web-based intervention of choice, after completing the six-month follow-up questionnaire. 


\section{Outcome measures}

\section{Clinical outcome measures}

The primary clinical outcome for the CEA was severity of depressive symptoms as measured by the Dutch version of the Center of Epidemiological Studies - Depression Scale (CES-D). The CES-D consists of 20 items (range o-6o) and measures symptoms of depression in the general population. Respondents rate to what extent they have experienced depressive symptoms in the previous week. Higher scores indicate more depressive symptoms (Haringsma et al., 2004; Radloff, 1977), and a score of 16 or higher is used as a cut-off level for clinically relevant depressive symptoms (Beekman et al., 1997; Smit et al., 2006).

For the CUA health-related quality of life was measured by the EuroQol - 5 Dimension (EQ-5D) of the EuroQol group. The EQ-5D consists of five health state dimensions (mobility, self-care, usual activities, pain/discomfort, and anxiety/ depression). Respondents have to indicate his or her own health state on a three level scale: no problems, some problems, and extreme problems. Quality of life is expressed by calculating an overall utility score that refers to the value that individuals or society may place on a particular health state. The utility is anchored between $\circ$ (death) and 1 (full health). This study used the Dutch tariff to value health states (Lamers et al., 2005). The utility scores are used to calculate quality adjusted lifeyears (QALYs) by weighing the amount of time spend in a particular health condition by the utility.

\section{Economic evaluation measures}

The health-economic analyses adopted the societal perspective encompassing 1) intervention costs, 2) costs for health care use, 3) participant's out of pocket payments, and 4) costs stemming from productivity losses. The web-based ACT intervention was compared with the web-based EW intervention and the WLC. Data was collected with the Trimbos and Institute of Medical Technology Assessment Questionnaire for Costs for Psychiatry (TIC-P; Hakkaart-Van Roijen et al., 2002) for three periods: the month prior to randomisation, and the months prior to the 6 and 12 months follow-up. The TIC-P asks respondents to note down the number of contacts with health care providers, the type of medication used, and the number of days absent of work and work cutback days with lesser efficiency while at work. The TIC-P is widely used as a measure of quantifying the economic costs of health care uptake and productivity losses, and is a valid and reliable instrument for people with mild to moderate health problems (Bouwmans et al., 2013). The cumulative costs were calculated using the mean area under the curve method (Matthews et al., 1990): $\left(T_{2}-T_{1}\right) \times\left(C_{1}+C_{2}\right) / 2+\left(T_{4}-T_{3}\right) \times\left(C_{3}+C_{4}\right) / 2$, where the time interval between 
the measurement points $(T)$ is 6 months and these periods of time are weighted by the average cost $(C)$ of the corresponding measurements. The various cost types are presented in more detail below.

\section{Intervention costs}

The per-participant costs of the interventions were calculated assuming that in the future the web-based ACT intervention would be offered in the community care setting. The per-participant intervention costs were estimated to be $€ 483$ for ACT and $€ 314$ for EW, consisting of licence costs, leisure time costs, counselling costs, and costs for hosting, maintenance and upgrading the intervention. For a comprehensive description of the calculation of the costs, see Appendix B.

\section{Health care costs}

The costs related to healthcare service uptake consisted of the number of health care units (e.g. sessions at a psychologist or psychiatrist, visits to the general practitioner or company doctor, appointments with a physiotherapist), multiplied by their standard cost price, according to the Dutch guidelines for health-economic evaluations (Hakkaart-Van Roijen et al., 2010). These costs were originally calculated for the reference year 2009, but were indexed for the year 2011 based on the Statistics Netherlands consumer price index (Table 1). To these were added the costs of medication (antidepressants, anxiolytics and hypnotics), calculated as the cost price per standard daily dose, as reported in the Dutch Pharmacotherpeutic Compass (Zorginstituut Nederland, 2014b) and the Dutch Health Care Insurance Board (Zorginstituut Nederland, 2014a), including $6 \%$ tax (not deductible by patients), and multiplied by the number of prescription days, plus the pharmacist's dispensing costs of $€ 6$ per prescription.

\section{Participants' out-of-pocket costs}

The patient's out of pocket costs (direct non-medical costs) are limited to the costs of making trips to and from health services. These costs were calculated as the average distance to a health service provider multiplied by the costs per $\mathrm{km}(€ 0.20)$ plus the costs for parking (€3).

\section{Costs owing to productivity losses}

Productivity losses occur when people do not go to their work (absenteeism) and when their productivity is reduced while at work (presenteeism). The corresponding costs were calculated according to a human capital approach (Rice \& Cooper, 1967). The mean productivity costs per hour per paid employer were $€ 34.75$ (Zorginstituut Nederland, 2015). 
Table 1 Direct medical and direct non-medical costs by health service type.

\begin{tabular}{|c|c|c|c|c|}
\hline \multirow[b]{2}{*}{ Health service type } & \multicolumn{2}{|c|}{$\begin{array}{l}\text { Direct medical costs } \\
\text { (in } 2011 € \text { ) }\end{array}$} & \multicolumn{2}{|c|}{$\begin{array}{l}\text { Direct nonmedical costs } \\
\text { (in } 2011 € \text { ) }\end{array}$} \\
\hline & Unit & Unit cost price ${ }^{a}$ & $\mathrm{Km}^{\mathrm{b}}$ & Unit cost price \\
\hline General practitioner - standard consult & Contact & 29.02 & 1.1 & 3.22 \\
\hline Company doctor or social worker & Contact & 67.37 & 5.0 & 4 \\
\hline Physiotherapist & Contact & 37.31 & 2.2 & 3.44 \\
\hline $\begin{array}{l}\text { Private practice psychiatrist, } \\
\text { psychotherapist, psychologist }^{c}\end{array}$ & Contact & 89.83 & 7.0 & 4.4 \\
\hline Community mental health service $^{d}$ & Contact & 179.30 & 7.0 & 4.4 \\
\hline Alternative treatment ${ }^{e}$ & Contact & 52.55 & 5.0 & 4 \\
\hline Home care & Hour & 36.28 & NA & NA \\
\hline Informal care (family, friends, self-help) ${ }^{f}$ & Hour & 12.96 & NA & NA \\
\hline
\end{tabular}

Note: NA, not applicable.

${ }^{a}$ Integral unit cost prices indexed from 2009 to the year 2011 (with consumer price index obtained from Statistics Netherlands).

${ }^{b}$ Based on average distances $(\mathrm{km})$ for receiving treatment, multiplied by the costs per $\mathrm{km}(€ \mathrm{\epsilon}, 20)$ and added with parking costs $(€ 3)$.

c Valued as the mean cost of professionals.

d Psychiatrist, psychotherapist and psychologist in community mental health service.

e Valued as average of Cesar/Mensendieck therapy, acupuncturist and homeopath (Hakkaart-van Roijen et al., 2010).

f Valued as domestic help (Hakkaart-van Roijen et al., 2010).

\section{Analyses}

Data were analysed in agreement with the Intention-to-treat (ITT) principle. ITT analyses were performed using the SPSS Missing Value Analysis to impute all missing data on the continuous measures with the expectation-maximization (EM) method (Dempster et al., 1977). Available data was $96.6 \%$ at baseline, $84.5 \%$ post-treatment, $86.9 \%$ at 6 -month follow-up, and $88.2 \%$ at 12 -month follow-up. Overall, significance was tested at $p<0.05$. The analyses were performed using SPSS 20 and Stata 13.

\section{Analysis of clinical and generic outcomes}

Treatment response was defined as clinically significant change (Jacobson and

Truax, 1991) in the depressive CES-D symptoms. First, the reliable change was calculated with the Reliable Change Index (RCI). Second, the recovery criterion was 
defined as a post-treatment score below the cut-off value of 16 for clinically relevant depressive symptoms. The score of 16 has been found in previous research as the cut-off score to indicate the presence of clinically relevant depressive symptoms (Beekman et al., 1997; Smit et al., 2006). A clinically significant change on the CES-D is thus defined as having a reliable change between the measurements and having a post-treatment score below the cut-off of 16 . Participants that had a clinically relevant change were either coded 1 (implying favourable treatment response, 'success') or o ('failure').

Quality adjusted life years (QALYs) were calculated using the mean area under the curve method (Matthews et al., 1990): $\left(T_{2}-T_{1}\right) \times\left(U_{1}+U_{2}\right) / 2+\left(T_{4}-T_{3}\right) \times\left(U_{3}+U_{4}\right) / 2$, where the time interval between the measurement points $(T)$ is 6 months and these periods of time are weighted by the average utility $(U)$ of the corresponding measurements. The QALY allows comparison of conditions over time in terms of average changes in health related quality of life. Statistical differences were calculated with Kruskal-Wallis H Test.

\section{Cost-effectiveness and cost-utility analysis}

The economic evaluation consisted of both a cost-utility analysis and a costeffectiveness analysis. In each case, the incremental cost-effectiveness ratio (ICER) was determined. The ICER was calculated as $\left(C_{1}-C_{0}\right) /\left(E_{1}-E_{0}\right)$, where $C$ are the average per-participant costs and $E$ is the effect, and the subscripts 1 and o refer to the intervention and control group respectively. The cost-effectiveness ICER indicates the costs per clinically relevant change in depressive symptoms. The cost-utility ICER indicates costs per QALY gained. Baseline differences between the ACT intervention and the two control conditions on the number of days that people were absent from their work prompted baseline-adjusted cost-effectiveness analyses. To handle stochastic uncertainty in the ICER, non-parametric bootstrapping was conducted to produce a scatter of 5000 simulated ICERs over the so called cost-effectiveness plane, with differences in costs on the vertical axis and differences in effects on the horizontal axis. Therefore, four quadrants appear. When the simulated ICERs are mainly in the south-east (SE) quadrant, more effect is gained for lower costs by the intervention relative to the comparator condition. This is the most favourable (dominant) outcome. In the north-west (NW) quadrant less effect is gained for additional costs (inferior quadrant), and in the south-west quadrant less effect is gained for lower costs. When the ICERs appear in the north-east (NE) quadrant, more effect is gained for additional costs. The additional health gains then need to be weighed against the additional costs. To decide whether higher costs outweigh the effect, a cost-effectiveness acceptability curve is generated. This acceptability curve represents the probability that the intervention is deemed cost-effective relative to 
the control condition given varying ceilings for the willingness to pay (WTP) for one case of depression showing clinically significant improvement or for gaining one quality-adjusted-life-year.

\section{Sensitivity analysis}

The analyses were repeated with lower intervention costs, assuming that the intervention is implemented in primary care with automated (not human) support, instead of human support. Results from a parallel study by Kelders et al. (2015) on the same web-based ACT intervention showed that there was no difference in the level of improvement from baseline to follow-up between the automated support and human support. Therefore intervention costs were recomputed by subtracting the costs of counselling. The costs were further lowered by excluding the Euro-value placed on leisure time, because the recent Dutch health-economic guideline assumes that the value of leisure time of the participants is already incorporated in the QALY, and double counting should be avoided (Zorginstituut Nederland, 2015). The intervention costs resulted therefore in a total of $€ 81$ for both interventions.

\section{Results}

\section{Participants' characteristics}

The mean age of participants was 46.8 years, and the sample was predominantly female $(76 \%)$ with a bachelor or above level of education (66.5\%). About two thirds of the participants had a paid job (65.8\%). The mean baseline score on the CES-D was 26.73 ( $\mathrm{sd}=8.38$ ), indicating a significant level of depressive symptoms. Of the 236 participants, 95 participants (40.3\%) were diagnosed with a mood disorder. Mean utility score on the EQ-5D was $0.72(s d=0.18)$. There were no significant differences at baseline between ACT versus EW and WLC on demographics, clinical outcomes, quality of life, or economic differences, except for absenteeism $\left[X^{2}(1, n=169)=7.369\right.$, $p<0.01$ for ACT versus WLC]. Closer inspection of the data revealed that there were only differences in the number of days absent from work (range ACT 1 - 16, EW 1- 25, WLC $1-8$ ), but no differences between the conditions for number of people having a paid job or number of people indicating absenteeism. At baseline, mean total costs per capita were $€ 590$, $€ 596$, and $€ 419$ for the ACT intervention, the EW intervention, and the WLC respectively (Table 2). Adherence to the intervention (defined as completing at least 6 sessions) was $84 \%$ for the ACT intervention, compared to $76 \%$ for the EW intervention. There were no significant differences between completers and non-completers with regard to demographic variables and outcome measures, and per-protocol analysis revealed similar outcomes (Pots et al., 2015). 
Table 2. Per capita costs by condition and time at baseline (in Euro, 2011) ${ }^{a}$.

\begin{tabular}{|c|c|c|c|c|c|c|}
\hline \multirow[b]{3}{*}{ Direct medical costs ${ }^{b}$} & \multicolumn{6}{|c|}{ Mean costs (sd) } \\
\hline & \multicolumn{2}{|c|}{ ACT } & \multicolumn{2}{|c|}{ EW } & \multicolumn{2}{|c|}{ WLC } \\
\hline & 109 & (166) & 91 & (130) & 82 & (177) \\
\hline Direct non-medical costs & 5 & (7) & 5 & (7) & 6 & (13) \\
\hline Absenteeism & 266 & (639) & 305 & (849) & 115 & (375) \\
\hline Presenteeism & 209 & $(412)$ & 196 & $(377)$ & 217 & $(604)$ \\
\hline Total costs & 590 & (943) & 596 & (998) & 419 & $(748)$ \\
\hline
\end{tabular}

Note: Differences in totals are due to rounding.

a Costs are presented in mean and standard deviation, based on a monthly basis (cf. Hakkaart-van Roijen et al., 2010).

$\mathrm{b}$ Including medication.

\section{Incremental costs}

Table 3 presents the mean direct, indirect and total cumulative costs in the conditions over the 12-month period. For the WLC the costs were limited to 6 months. The larger share of the costs was attributable to the indirect costs, specifically absenteeism. Overall, the ACT intervention generated lesser costs than the EW intervention at 6 months, but more costs than the WLC. At 6 months follow-up the costs were $€ 3098$ ( $95 \% \mathrm{CI}, 2313-3884$ ) for the ACT intervention, and $€ 3405$ ( $95 \% \mathrm{CI}, 2071-4737$ ) and $€ 2380$ ( $95 \% \mathrm{CI}, 1530-3231)$ for EW and WLC respectively. Therefore, the incremental costs for ACT versus EW were $€ 3098-€ 3405=-€ 307$ (savings), indicating that the ACT intervention was less costly than the EW intervention. The costs at 12 months follow-up were almost equivalent between the two interventions: $€ 5492-€ 5427=$ $€ 65$. The incremental costs for ACT versus WLC were $€ 3098-€ 2380=€ 718$, indicating that the ACT intervention was more costly than the WLC at 6 months.

Table 4 presents the baseline-adjusted incremental costs (controlling for the number of days absent from work). All incremental costs need to be viewed with caution, because the study was not powered for testing hypotheses about cost differences. 


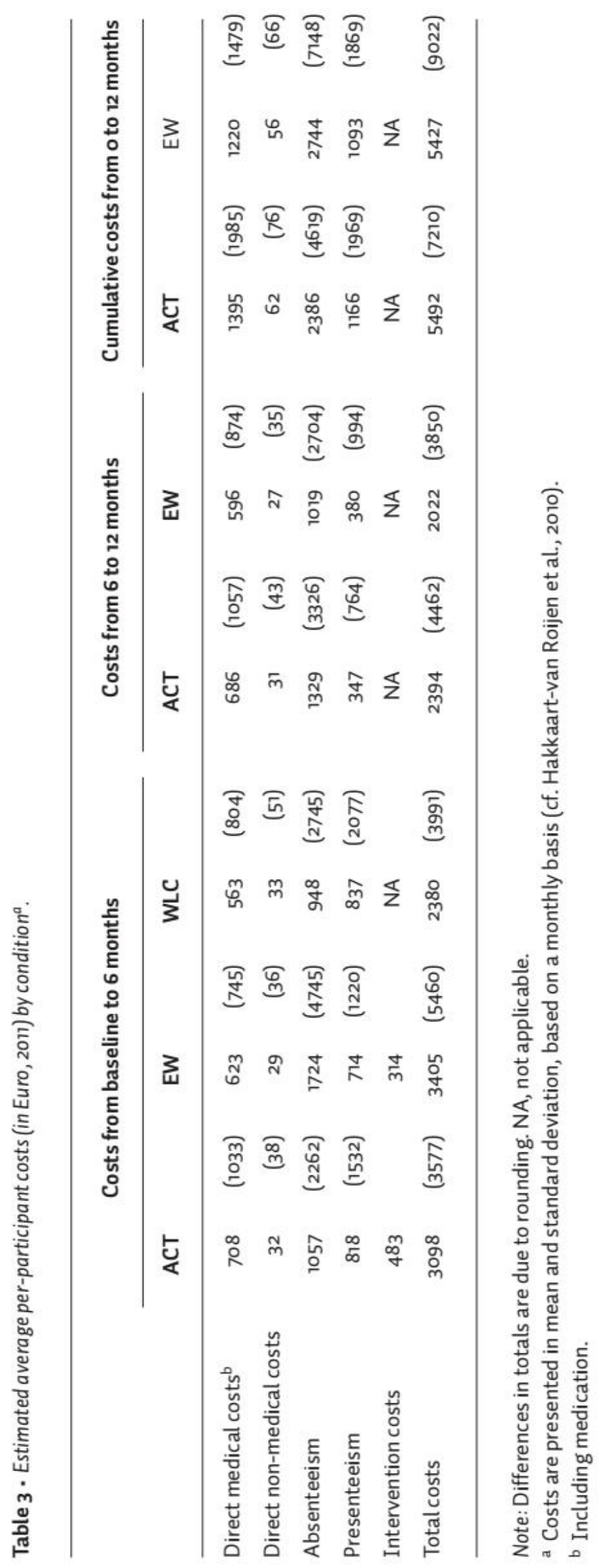




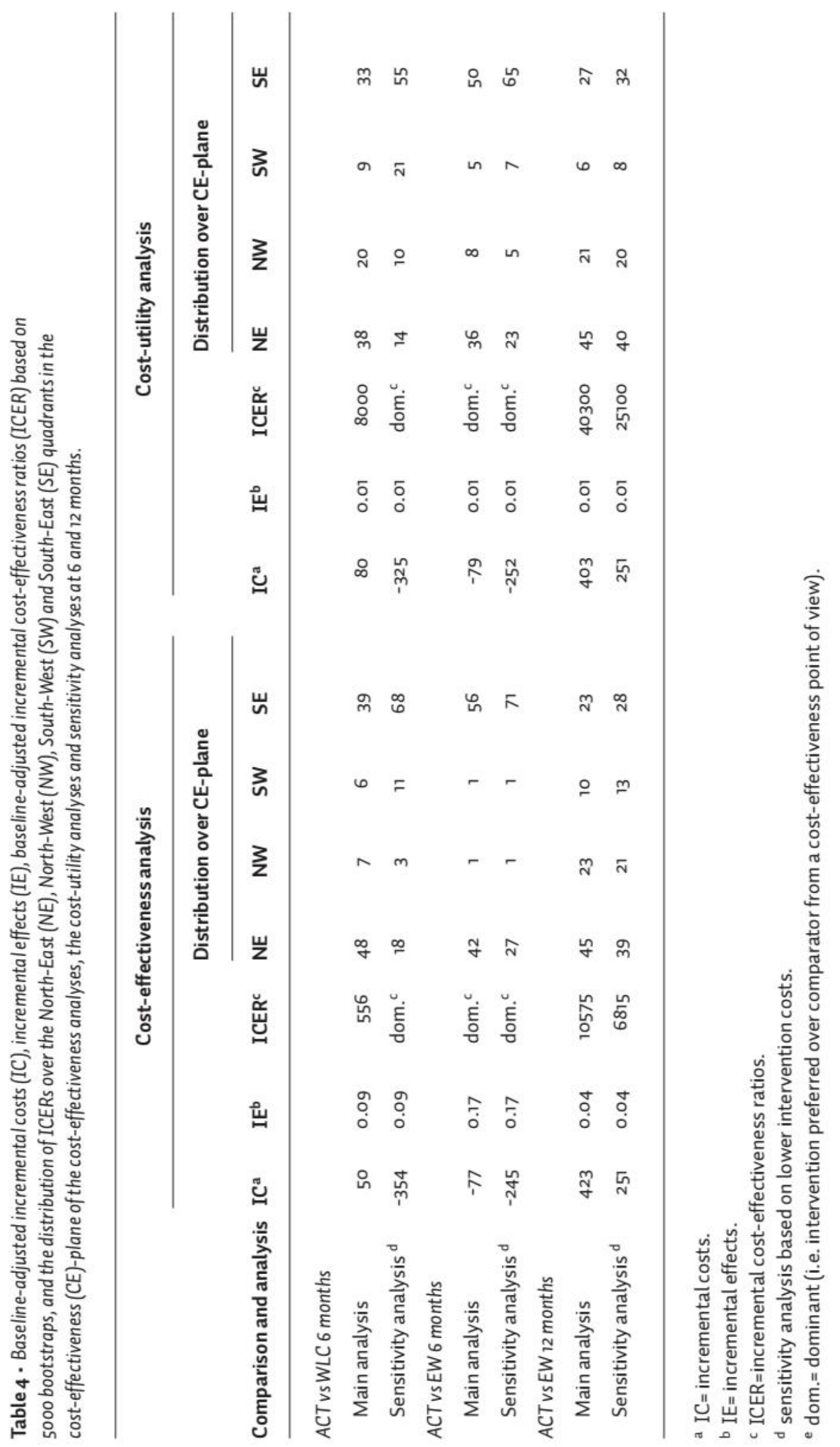




\section{Incremental effectiveness}

After six months, the proportion of participants that improved clinically significant on the CES-D in the ACT intervention was $43 / 82$ (0.524), compared to $38 / 87$ (0.437) in the WLC, and 24/67 (0.358) in the EW intervention. The incremental effectiveness was calculated as the difference between the probabilities of a beneficial outcome in each of the groups. For ACT versus WLC the incremental effect at 6 months was 0.524 $-0.437=0.087$ (95\% CI -0.645-0.240), not significant $\left(X^{2}{ }_{1}=1.30, p=0.255\right)$. For ACT versus $\mathrm{EW}$, the incremental effect was $0.524-0.358=0.166$ (95\% CI $0.005-0.327$ ), favouring the ACT intervention and statically significant $\left(\mathrm{X}_{1}{ }_{1}=4.12, \mathrm{P}<0.05\right)$. Its inverse, the number needed to treat was $1 / 0.166=6.02$, indicating that an additional clinically significant change was gained in one out of every six participants when people were referred to ACT rather than EW. At 12 months, the incremental effect of ACT versus $E W$ was $0.439-0.403=0.036$ (95\% CI -0.126-0.198). No significant differences were found between $\mathrm{ACT}$ and EW at 12 months $\left(\mathrm{X}^{2}{ }_{1}=0.20, \mathrm{P}=0.658\right)$.

The cumulative QALY gain over 6 months was 0.39 (95\% CI $0.37-0.41$ ) for the ACT intervention, compared to 0.38 (95\% CI 0.36 - 0.40) for the EW intervention, and 0.38 (95\% CI 0.37 - 0.40) for the WLC. The difference in QALY gains between ACT versus WLC was $0.39-0.38=0.01$ over 6 months, not significant $(H(1)=0.903, p=0.342)$. For ACT versus EW the QALY score at 6 months was $0.39-0.38=0.01$, favouring the ACT intervention but statistically not significant $(H(1)=2.313, P=0.128)$. At 12 months the QALY score was 0.787 (95\% CI 0.75 - 0.82) and 0.773 (95\% CI 0.74 - 0.81 ) for ACT versus EW, with a non-statistical difference in QALY gains of $0.787-0.773=0.01(\mathrm{H}(1)$ $=0.642, \mathrm{p}=0.423$ ).

\section{Cost-effectiveness}

\section{Results at 6 months}

Table 4 represents the baseline-adjusted mean incremental costs (IC), the incremental effects (IE), the baseline-adjusted ICERs, and the distribution of the ICERs over the cost-effectiveness plane. For ACT versus WLC the incremental costs were $€ 50$ and the incremental effect was 0.09 at 6 months, estimating a mean ICER $€ 50 / 0.09=€ 556$ for a treatment responder. This implies that by offering the ACT intervention instead of placing the participant on a waiting list, extra costs of $€ 556$ are incurred for a health gain of one additional clinically significant improved patient. Figure 1 shows that the majority of the simulated ICERs (48\%) appear in the north-east quadrant of the cost-effectiveness plane, indicating that the ACT intervention has a $48 \%$ likelihood to be more effective but also more costly than placing people on a waitlist. Additionally, there is a $39 \%$ probability that the ACT intervention is more effective at lower costs (dominant), a $7 \%$ probability of being less effective at more costs (inferior), and $6 \%$ 
less effective at lower costs. With no willingness to pay the probability of the ACT intervention being more cost-effective than the WLC is $45 \%$, which increases with a willingness to pay of $€ 10,000(81 \%)$, $€ 20,000(86 \%)$, and $€ 30,000(87 \%)$.

For ACT versus EW the incremental costs were - $€ 77$ at 6 months (savings), and the incremental effect 0.17 . This implies that the ACT intervention is dominant relative to the $\mathrm{EW}$ intervention. Figure 1 shows the scatterplot of the incremental cost-effectiveness plane, each dot indicating one simulated ICER of ACT versus EW at 6 months. The figure shows that the majority of the simulated ICERs appeared in the dominant south-east quadrant ( $56 \%$ ), which indicates that there is a $56 \%$ probability that the ACT intervention is more effective at lower costs. In addition, $42 \%$ of the ICERs fell in the north-east quadrant, indicating more effect at additional costs. The incremental cost-effectiveness acceptability curve suggest that when there is no willingness to pay for a clinically significant improvement in depression, there is a $57 \%$ probability that the ACT intervention is more cost-effective than the EW intervention. When the willingness to pay is $€ 10,000, € 20,000$ or $€ 30,000$, this probability rapidly increases to $96 \%, 98 \%$, and $98 \%$ respectively.
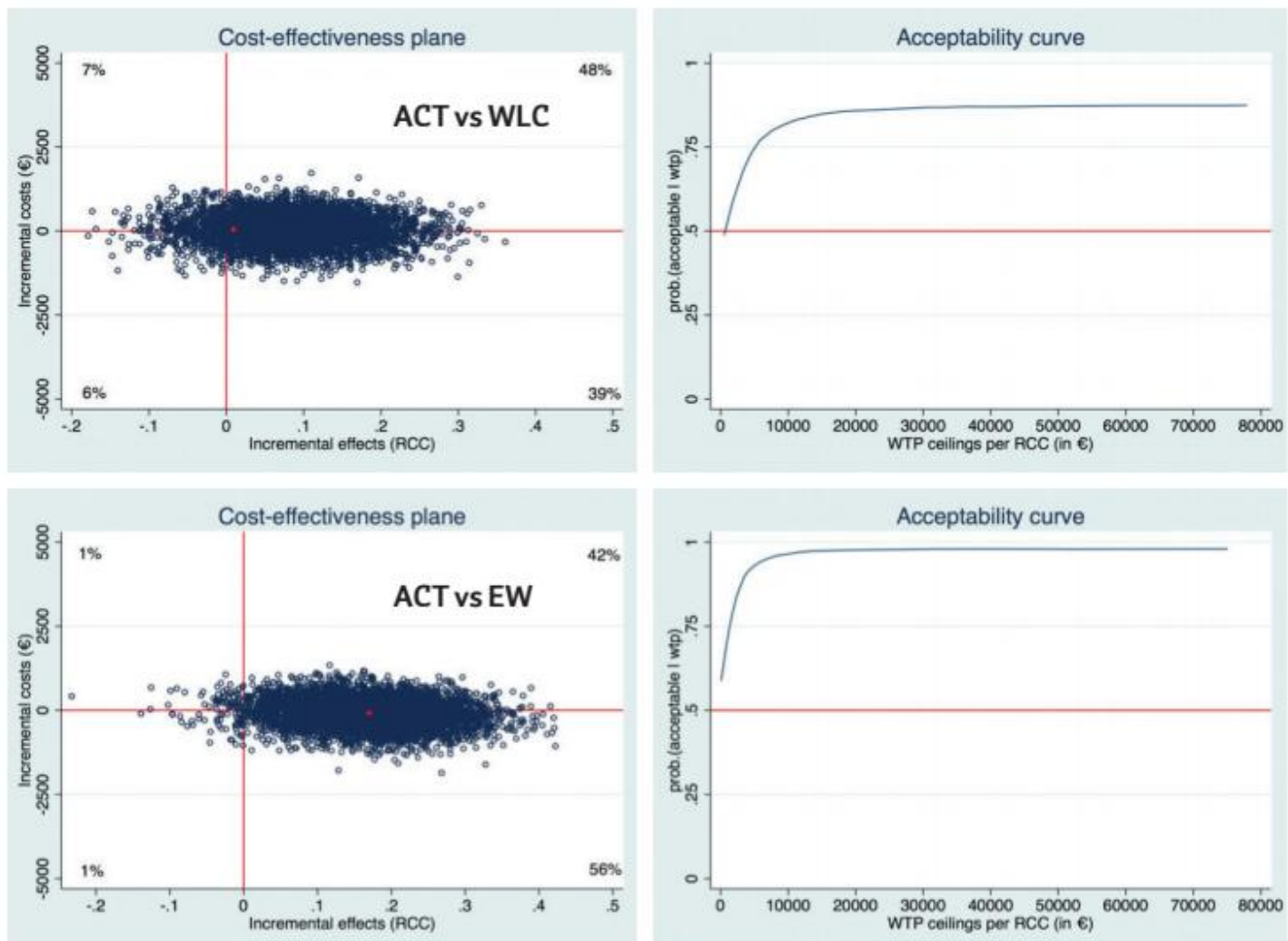

Figure 1 - Cost-effectiveness plane and acceptability curve with distribution of bootstrapped incremental cost-effectiveness ratios (ICERs) $(n=5000)$ for clinically significant change in depressive symptoms at 6 months. 

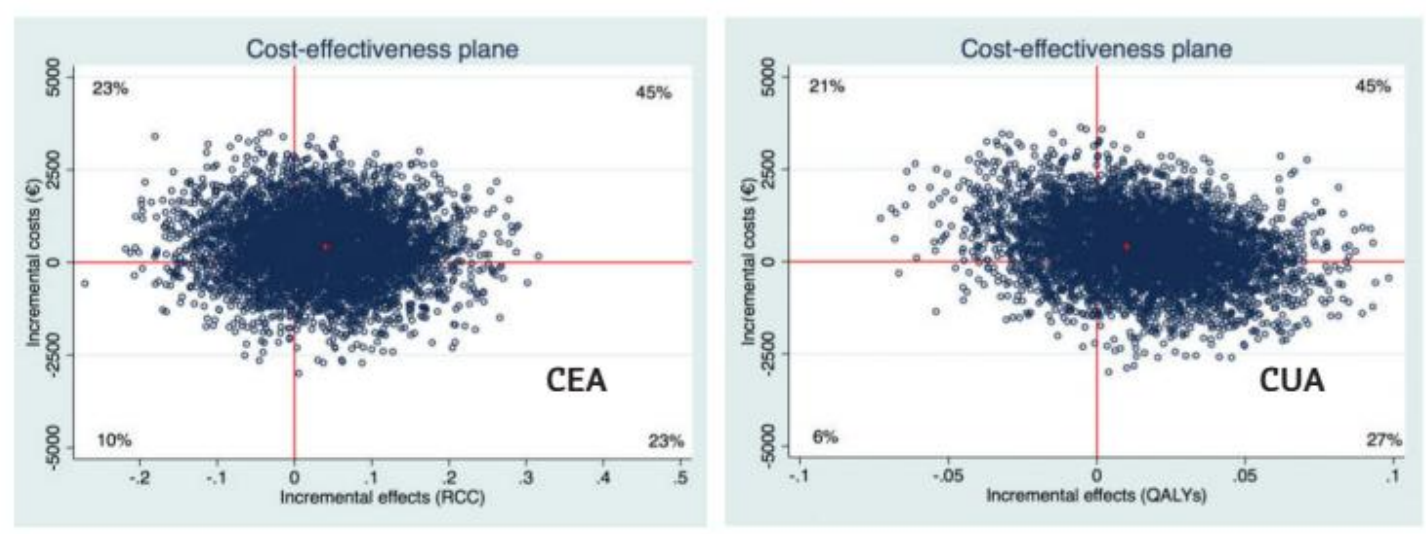

Figure 2 - Cost-effectiveness plane with distribution of bootstrapped incremental cost-effectiveness ratios (ICERs) ( $n=5000)$ for clinically significant change in depressive symptoms and quality of life at 12 months.

\section{Results at 12 months}

At 12 months the mean ICER of ACT versus EW was estimated at $€ 423 / 0.04=€ 10,575$ for a treatment responder. As is shown in Figure 2, the most dots fall in the north-east and south-east quadrant of the cost-effectiveness plane ( $45 \%$ and $23 \%$ respectively), indicating that the ACT intervention has a probability of being $23 \%$ more effective at lower costs and $45 \%$ more effective at additional costs. The figure also shows that there is a probability that the ACT intervention is less effective at higher costs ( $23 \%$ - inferior) and at lower costs (10\%). With no willingness to pay the probability of the ACT intervention being more cost-effective than EW is approximately $33 \%$, which steadily increases with a willingness to pay of $€ 10,000, € 20,000$, and $€ 30,000$, to $46 \%, 56 \%$ and $59 \%$ respectively.

\section{Cost-utility}

\section{Results at 6 months}

The mean incremental cost-effectiveness ratio for ACT versus WLC was $€ 8,000$, indicating that for offering ACT versus WLC $€ 8,000$ costs were incurred for one QALY gained. Figure 3 shows that $38 \%$ of all simulated dots of the cost-effectiveness plane appear in the north-east quadrant and $33 \%$ appear in the south-east quadrant, showing that there is a $33 \%$ probability that the ACT intervention is superior in costs and health, and a $38 \%$ probability of more effect at additional costs. However, there is also a $20 \%$ probability that ACT generated worse health against higher costs when compared to WLC. The acceptability curve shows that when there is no willingness to pay there is an $42 \%$ probability of ACT being more cost-effective, and that percentage slowly rises to $47 \%$ with a willingness of $€ 10,000$, to $53 \%$ at $€ 20,000$ and to $56 \%$ at $€ 30,000$. 
For ACT versus EW the mean ICER was $€-79$ (savings), indicating that the intervention must be regarded as the intervention of choice or dominant intervention, because ACT generated similar or more QALY health gains against fewer costs. Figure 3 shows the cost-effectiveness plane, which shows that about $50 \%$ of all simulated ICERs fall in the dominant south-east quadrant (more QALYs for fewer costs), and $36 \%$ of the ICERs were located in the north-east quadrant (more QALYs for additional costs). When there is no willingness to pay per gained QALY, the ACT intervention has an $55 \%$ probability of being more cost-effective compared to the EW intervention, rising to $68 \%, 75 \%$ and $80 \%$ at $€ 10,000$ and $€ 20,000$ and $€ 30,000$ respectively.
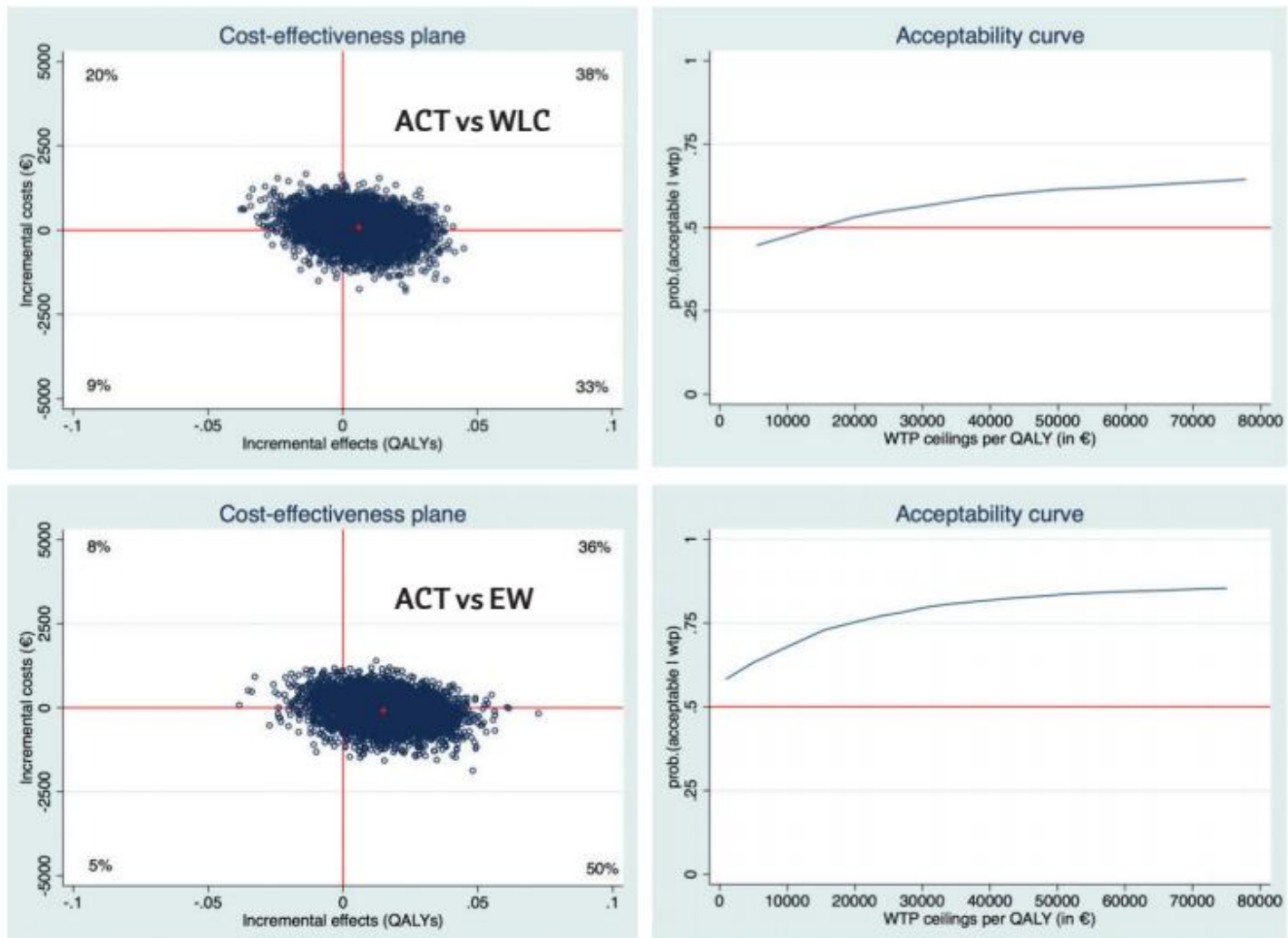

Figure 3 - Cost-effectiveness plane and acceptability curve with distribution of bootstrapped incremental cost-effectiveness ratios (ICERs) ( $n=5000)$ for quality of life at 6 months.

Results at 12 months

At 12 months the mean ICER was $€ 40,300$ for ACT versus EW. This means that at 12 months that by offering ACT versus EW $€ 40,300$ costs were incurred for one additional QALY gained. However, looking at the dots in the distribution plane, we see that they are relatively equally distributed in the cost-effectiveness plane ( $45 \%$ north-east, $27 \%$ south-east, $21 \%$ north-west, $6 \%$ south-east), indicating that there is a $45 \%$ probability 
that at 12 months ACT gains more QALYs for additional costs, $27 \%$ probability that ACT gains more QALYs against fewer costs, but also a 21\% probability that ACT generates worse health gains against higher costs compared to the $\mathrm{EW}$ intervention. The WTP starts at $33 \%$ with no willingness to pay, rising slowly to $40 \%, 46 \%$, and $51 \%$ with $€ 10,000, € 20,000$ and $€ 30,000$ respectively.

\section{Sensitivity analysis}

Sensitivity analyses were conducted by repeating the main CEA analyses with lower intervention costs of $€ 81$, excluding leisure time costs and diminishing counselling costs. For ACT versus WLC sensitivity analyses overall showed that the intervention produced better health outcomes at 6 months against costs savings ( $€-354$ and $€-325$ ), the ACT intervention being the dominant intervention both for cost-effectiveness and cost-utility. Distribution of the CE-plane shifted towards the south-east dominant quadrant. For ACT versus EW the sensitivity analysis showed a further cost savings at 6 months per health gain of both additional clinically significant change ( $€-245)$ and health-related quality of life ( $€-252)$. This indicates that when both interventions have the same (lower) intervention costs, the ACT intervention stays the dominant intervention. At 12 months there is a reduction in costs, indicating that the sensitivity analysis reduced the incremental costs by half ( $€ 251$ CEA; $€ 251$ CUA). Distribution of the CE-plane and probabilities at different WTP ceilings remained about the same. Overall, sensitivity analysis with lower intervention costs indicates that the web-based ACT intervention is the dominant intervention, because it generated better health at lower costs at 6 months.

\section{Discussion}

\section{Main findings}

We performed a health-economic evaluation alongside a three-armed pragmatic randomised controlled trial, to evaluate if the web-based ACT intervention would lead to increased health gains at lower costs. Cost-effectiveness and cost-utility analyses allow health care organisations and other stakeholders to compare benefits of interventions, in consideration of long waiting lists and the rapidly increasing costs of health care. Given this challenge, it is essential to assess cost-effectiveness and cost-utility of web-based interventions. Overall, the web-based ACT intervention was only successful at 6 months, compared to $E W$, in reducing depressive symptoms and increasing quality of life, being the dominant intervention. This effect recedes at 12 months, where ACT is no longer significantly more effective than EW. Compared to the WLC, the ACT intervention was not significantly more effective in increasing 
health gains at 6 months, and the likelihood of the web-based ACT intervention being superior was $39 \%$ and $33 \%$ for cost-effectiveness and cost-utility respectively.

Contrary to our expectations, the web-based ACT intervention was superior in comparison with the active control condition, but not compared to the WLC. The proportion of participants that reached a clinically significant change in depressive symptoms was higher in the ACT intervention group at 6 months ( $52 \%$ versus $36 \%$ EW and $44 \%$ WLC), but only statistically significant in the ACT versus EW comparison. Looking at the results of our study, it seems that the WLC also shows a reduction of depressive symptoms at 3 and 6 months. This reduction of depressive symptoms in the WLC was higher than seen in comparable studies on self-help ACT interventions (e.g. Fledderus et al., 2012; Bohlmeijer et al., 2011; Lappalainen et al., 2015; Räsänen et al., 2016; Trompetter et al., 2015). Results from The Netherlands Mental Health Survey and Incidence Study (NEMESIS; Spijker et al., 2002) showed that 50\% (95\% CI $44-56 \%$ ) recovered within 3 months, and $63 \%$ (95\% CI $57-69 \%)$ within six months. Also, Kruijshaar et al. (2005) showed that the average mean duration of depression was 6 months. As many depressive episodes remit spontaneously, not all depressed individuals necessarily need prompt treatment (Spijker and Nolen, 1998; Spijker et al., 2002). This could lead to the conclusion that it is worthwhile to wait when having depressive symptoms instead of offering the web-based ACT intervention. However, the results of our clinical trial showed that over time (o to 6 months) the web-based ACT intervention was more effective than both control conditions, with additional analyses showing that the largest effect was found at 3 months after baseline (Pots et al., 2015). This suggests that although the WLC is similar in effect at 6 months, the web-based ACT intervention is clinically more effective earlier in time. In line with the revised Dutch guidelines for evidence-based treatment (Mental Health Guidelines, www.ggzrichtlijnen.nl), web-based interventions (bibliotherapy or guided web-based interventions) can be offered as first-step interventions in the first three months, parallel to watchful waiting (Spijker et al., 2013).

For ACT versus EW, the CEA showed that the web-based ACT intervention is the dominant intervention, and has a $96 \%$ probability of being more cost-effective with a willingness to pay ceiling of $€ 10,000$ placed on a clinically significant change in depressive symptoms. Likewise, the CUA demonstrated that with no willingness to pay the ACT intervention has an $55 \%$ probability of being more cost-effective compared to the $\mathrm{EW}$ intervention, rising to $68 \%, 75 \%$ and $80 \%$ at $€ 10,000$ and $€ 20,000$ and $€ 30,000$ respectively for gaining one QALY. Follow-up analyses at 12 months revealed only a $23 \%$ and $27 \%$ probability of being the superior intervention for a clinically significant change in depression and a QALY health gain respectively, indicating that both interventions are similar in cost-effectiveness at 12 months.

With lower intervention costs, sensitivity analyses showed that ACT was the 
dominant intervention compared to both control conditions. Compared to the WLC, it seems that with lower interventions costs ACT produces cost savings, even though ACT has no offset in clinical effectiveness. Compared to EW, with lower (equal) intervention costs, ACT remains the dominant intervention. These results are important as the overall expenditure for health care is increasing. The web-based ACT intervention is at present in The Netherlands implemented in community care with automated support, thus lowering the costs of offering the web-based ACT intervention. Pro-actively offering web-based ACT could be a feasible solution, since web-based ACT generates clinical effectiveness at an earlier point in time, is scalable, and would lower the per-patient health care costs. Ultimately, to decrease the burden of depression by public mental health interventions, from a health-economic perspective one could question the above and still decide to place a person at risk on the waiting list. The results, however, remain puzzling looking at the results of both control conditions. As EW has small effects (Frattaroli, 2004), there is a possibility that the web-based EW intervention is clinically less effective than WLC. The results from our clinical trial, on the other hand, showed that both control conditions were not significantly different in outcome measures from baseline to 6-month follow-up. This suggests that probably another process is at play. One possibility, looking at the design of our study, is that the expectation of the WLC of starting the intervention directly after the assessment at 6 months induced a positive mood, resulting in reporting better health outcomes. Also, as our study was not powered for economic analyses or reporting differences in clinically significant change, it is possible that the differences between the conditions was too small. Considering the probabilistic approach of our study, it seems that, although the web-based ACT intervention is more effective at 3 months, at 6- and 12-months follow-up there is no large costeffectiveness gain compared to both control conditions.

To our knowledge, this is the first economic evaluation of a web-based ACT intervention for depression. In line with recent meta-analyses showing that ACT can be effective for depression (A-Tjak et al., 2015; Hacker et al., 2016; Öst, 2014; Ruiz, 2010), our clinical trial showed that ACT was over time more effective than both control conditions (Pots et al., 2015). However, this economic evaluation indicates that the web-based ACT intervention is only cost-effective from a societal point of view compared to the EW intervention. Cost-effectiveness studies on Internet-based Cognitive Behaviour Therapy (ICBT) for depression (Arnberg et al., 2014), and mental disorders (Donker et al., 2015; Hedman et al., 2012; Mihalopoulos and Chatterton, 2015) show that ICBT can be cost-effective compared to no treatments or conventional CBT. Hedman et al. (2012) suggested in their systematic review that ICBT had more than a $50 \%$ probability of being more cost-effective compared to no treatment or to conventional CBT when willingness to pay for an additional improvement was 
zero. This compares well with the results in our study, at least for the comparison with the active control group ( $56 \%)$. This is an important finding, as the presence of clinically relevant depressive symptoms is known to be a risk factor for clinical depression (Cuijpers and Smit, 2004), the results of our study could suggest that the web-based ACT intervention has potential to decrease the risk of a new episode of depression sooner than both control conditions. However, the long-term results from our study show that the health-economic gains are limited, and at present no final conclusions about the benefits of the implementation of web-based ACT as a means to lessen the burden on depression can be made. It is recommended to replicate the study, using no treatment or conventional ACT as control group as has been done in similar studies with iCBTs.

In conclusion, the results of this study suggest that web-based ACT could be worthwhile to implement with lower intervention costs from the perspective of clinical effectiveness on the short-term. However these results are exploratory and need further research, especially with regard to the longer-term cost-effectiveness.

\section{Strengths and limitations}

The main strength of this study is the three-armed pragmatic randomised controlled trail, where non-specific treatment factors are taken into account. Also, adherence in our study was high ( $84 \%)$, which is of importance as non-adherence is called one of the main reasons for the low impact of web-based interventions (Christensen et al., 2009; Donkin et al, 2011; Eysenbach, 2005; Kelders et al., 2012). Furthermore, to enhance generalisability to the general population and strengthen the external validity of our study, our inclusion criteria were kept broad. Moreover, for cost-effectiveness studies it is important to lengthen the time-span of the trial to a year or longer. In our trial we expanded the time-span of the ACT versus EW comparison to 12 months, which permits the assessment of the longer-term cost-effectiveness and the cost-effectiveness over time.

Some limitations also apply. First, the study was not powered to test cost-differences between conditions, therefore we applied a probabilistic decision-making approach and the results must be considered explorative. Second, although inclusion criteria were kept broad to strengthen the external validity of our trial, our recruitment strategy raises the possibility of self-selection bias. Participants were mostly women with a high level of education, which questions the representativeness of our sample and generalisability of our findings to other groups. Third, the fact that the participants could not be blinded to the condition they were assigned to, might have distorted the results of our study. Because of these limitations, the results of our study must be considered with some caution. 


\section{References}

A-Tjak, J. G. L., Davis, M. L., Morina, N., Powers, M. B., Smits, J. A. J. \& Emmelkamp, P. M. G. (2015). A meta-analysis of the efficacy of acceptance and commitment therapy for clinically relevant mental and physical health problems. Psychotherapy and Psychosomatics 84, 30-36.

Arnberg, F. K., Linton, S. J., Hultcrantz, M., Heintz, E. \& Jonsson, U. (2014). Internet-delivered psychological treatments for mood and anxiety disorders: A systematic review of their efficacy, safety, and cost-effectiveness. PLoS ONE 9, eg8118.

Beekman, A. T. F., Deeg, D. J. H., Van Limbeek, J., Braam, A. W., De Vries, M. Z. \& Van Tilburg, W. (1997). Criterion validity of the Center for Epidemiologic Studies Depression scale (CES-D): Results from a community-based sample of older subjects in the Netherlands. Psychological Medicine 27, 231-235.

Bohlmeijer, E. T., Fledderus, M., Rokx, T. A. J. J. \& Pieterse, M. E. (2011). Efficacy of an early intervention based on acceptance and commitment therapy for adults with depressive symptomatology: Evaluation in a randomized controlled trial. Behaviour Research and Therapy 49, 62-67.

Bouwmans, C., De Jong, K., Timman, R., Zijlstra-Vlasveld, M., Van Der Feltz-Cornelis, C., Tan, S. S. \& Hakkaart-Van Roijen, L. (2013). Feasibility, reliability and validity of a questionnaire on healthcare consumption and productivity loss in patients with a psychiatric disorder (TiC-P). BMC Health Services Research 13, 217.

Christensen, H., Griffiths, K. M. \& Farrer, L. (2009). Adherence in internet interventions for anxiety and depression. Journal of Medical Internet Research 11, e13.

Cook, R. J. \& Sackett, D. L. (1995). The number needed to treat: A clinically useful measure of treatment effect. British Medical Journal 310, 452-454.

De Graaf, R., Ten Have, M., Van Gool, C. \& Van Dorsselaer, S. (2012a). Prevalence of mental disorders and trends from 1996 to 2009 . Results from the Netherlands Mental Health Survey and Incidence Study-2. Social Psychiatry and Psychiatric Epidemiology 47, 203-213.

De Graaf, R., Tuithof, M., Van Dorsselaer, S. \& Ten Have, M. (2012b). Comparing the effects on work performance of mental and physical disorders. Social Psychiatry and Psychiatric Epidemiology 47, 1873-1883.

Dempster, A. P., Laird, N. M. \& Rubin, D. B. (1977). Maximum likelihood from incomplete data via the EM algorithm. Journal of the Royal Statistical Society Series B Methodological 39, 1-38.

Donker, T., Blankers, M., Hedman, E., Ljotsson, B., Petrie, K. \& Christensen, H. (2015). Economic evaluations of Internet interventions for mental health: A systematic review. Psychological Medicine 45, 3357-3376.

Donkin, L., Christensen, H., Naismith, S. L., Neal, B., Hickie, I. B. \& Glozier, N. (2011). A Systematic Review of the Impact of Adherence on the Effectiveness of e-Therapies. Journal of Medical Internet Research 13, e52.

Eysenbach, G. (2005). The law of attrition. Journal of Medical Internet Research 7, e11.

Fledderus, M., Bohlmeijer, E. T., Pieterse, M. E. \& Schreurs, K. M. G. (2012). Acceptance and commitment therapy as guided self-help for psychological distress and positive mental health: A randomized controlled trial. Psychological Medicine 42, 485-495.

Fledderus, M., Bohlmeijer, E. T., Smit, F. \& Westerhof, G. J. (2010). Mental health promotion as a new goal in public mental health care: A randomized controlled trial of an intervention enhancing psychological flexibility. American Journal of Public Health 100, 2372-2378.

Frattaroli, J. (2006). Experimental disclosure and its moderators: A meta-analysis. Psychological Bulletin 132, 823-865.

Gross JJ, Thompson RA. Emotion regulation: conceptual foundations. In: Gross JJ, editor. Handbook of Emotion Regulation. New York: Guilford Press; 2011

Hacker, T., Stone, P. \& Macbeth, A. (2016). Acceptance and commitment therapy - Do we know enough? Cumulative and sequential meta-analyses of randomized controlled trials. Journal of Affective Disorders 190, 551-565. 
Hakkaart-Van Roijen, L., Tan, S. S. \& Bouwmans, C. A. M. (2010). Handleiding voor kostenonderzoek methoden en standaard kostprijzen voor ecenomische evaluaties in de gezondheidszorg [Manual for costing research - In Dutch, actualized version 2010]. Institute for Medical Technology Assessment, Erasmus University: Rotterdam.

Hakkaart-Van Roijen, L., Van Straten, A. \& Donker, M. (2002). Trimbos/iMTA questionnaire for costs associated with psychiatric illness (TIC-P). iMTA: Rotterdam.

Haringsma, R., Engels, G. I., Beekman, A. T. F. \& Spinhoven, P. (2004). The criterion validity of the Center for Epidemiological Studies Depression Scale (CES-D) in a sample of self-referred elders with depressive symptomatology. International Journal of Geriatric Psychiatry 19, 558-563.

Hayes, S. C., Luoma, J. B., Bond, F. W., Masuda, A. \& Lillis, J. (2006). Acceptance and Commitment Therapy: Model, processes and outcomes. Behaviour Research and Therapy 44, 1-25.

Hayes, S. C., Strosahl, K. D. \& Wilson, K. G. (2012). Acceptance and Commitment Therapy: the Process and Practice of Mindful Change. Guilford Press: New York.

Hedman, E., Ljótsson, B. \& Lindefors, N. (2012). Cognitive behavior therapy via the Internet: A systematic review of applications, clinical efficacy and cost-effectiveness. Expert Review of Pharmacoeconomics and Outcomes Research 12, 745-764.

Jacobson, N. S. \& Truax, P. (1991). Clinical significance: A statistical approach to defining meaningful change in psychotherapy research. Journal of Consulting and Clinical Psychology 59, 12-19.

Kelders, S. M., Bohlmeijer, E. T., Pots, W. T. M. \& van Gemert-Pijnen, J. E. W. C. (2015). Comparing human and automated support for depression: Fractional factorial randomized controlled trial. Behaviour Research and Therapy 72, 72-80.

Kelders, S. M., Pots, W. T., Oskam, M. J., Bohlmeijer, E. T. \& Van Gemert-Pijnen, J. E. (2013). Development of a web-based intervention for the indicated prevention of depression. BMC Medical Informatics and Decision Making 13, 1-11.

Kelders, S. M., Kok, R. N., Ossebaard, H. C. \& Van Gemert-Pijnen, J. E. W. C. (2012). Persuasive system design does matter: A systematic review of adherence to web-based interventions. Journal of Medical Internet Research 14, e152.

Kruijshaar, M. E., Barendregt, J., Vos, T., Graaf, R. de, Spijker, J., \& Andrews, G. (2005). Lifetime prevalence estimates of major depression: an indirect estimation method and a quantification of recall bias. European Journal of Epidemiology, 20, 103-111.

Lamers, L. M., Stalmeier, P. F. M., McDonnell, J., Krabbe, P. F. M. \& Van Busschbach, J. J. (2005). Measuring the quality of life in cost-utility analyses: The Dutch EQ-5D tariff. Nederlands Tijdschrift voor Geneeskunde 149, 1574-1578.

Lappalainen, P., Langrial, S., Oinas-Kukkonen, H., Tolvanen, A., \& Lappalainen, R. (2015). Web-Based Acceptance and Commitment Therapy for Depressive Symptoms With Minimal Support : A Randomized Controlled Trial. Behavior Modification, 39, 805-834.

Leon, A. C., Olfson, M., Portera, L., Farber, L. \& Sheehan, D. V. (1997). Assessing psychiatric impairment in primary care with the Sheehan Disability Scale. International Journal of Psychiatry in Medicine 27, 93-105.

Mathers, C. D. \& Loncar, D. (2006). Projections of global mortality and burden of disease from 2002 to 2030. PLoS Medicine 3, 2011-2030.

Matthews, J. N. S., Altman, D. G., Campbell, M. J. \& Royston, P. (1990). Analysis of serial measurements in medical research. British Medical Journal 300, 230-235.

Mihalopoulos, C. \& Chatterton, M. L. (2015). Economic evaluations of interventions designed to prevent mental disorders: A systematic review. Early Intervention in Psychiatry 9, 85-92.

Öst, L. G. (2014). The efficacy of Acceptance and Commitment Therapy: An updated systematic review and meta-analysis. Behaviour Research and Therapy 61, 105-121.

Pennebaker, J. W. (1997). Writing about emotional experiences as a therapeutic process. Psychological Science 8, 162-166.

Pots, W. T. M., Fledderus, M., Meulenbeek, P. A. M., Klooster ten, P. M., Schreurs, K. M. \& Bohlmeijer, E. T. (2015). Acceptance and commitment therapy as a web-based intervention for depressive symptoms: randomised controlled trial. British Journal of Psychiatry 208, 69-77. 
Radloff, L. S. (1977). The CES-D scale, a self report depression scale for reseach in the general population. Applied Psychological Measurement 1, 385-401.

Räsänen, P., Lappalainen, P., Muotka, J., Tolvanen, A. \& Lapplalainen, R. (2016). An online guided ACT intervetnion for enhancing the psychological wellbeing of university students: a randomized controlled clinical trial. Behaviour Research and Therapy 78, 30-42.

Rice, D.P. \& Cooper, B.S. (1967). The economic value of human life. American Journal of Public Health and the Nation's Health 57, 1954-1966.

Riper, H., van Ballegooijen, W., Kooistra, L., de Wit, J. \& Donker, T. (2013). Preventie \& eMental-health. Onderzoek dat leidt, technologie die verleidt, preventie die bereikt en beklijft. Vrije Universiteit Amsterdam: Amsterdam.

Ruiz, F. J. (2010). A review of acceptance and commitment therapy (ACT) empirical evidence: Correlational, experimental psychopathology, component and outcome studies. International Journal of Psychology and Psychological Therapy 10, 125-162.

Sheehan, D. V., Lecrubier, Y., Sheehan, K. H., Amorim, P., Janavs, J., Weiller, E., . . Dubar, G. C. (1998). The Mini-International Neuropsychiatric Interview (M.I.N.I.): the development and validation of a structured diagnostic psychiatric interview for DSM-IV and ICD-10. Journal of Clinical Psychiatry 59, 22-33.

Smit, F., Ederveen, A., Cuijpers, P., Deeg, D. \& Beekman, A. (2006). Opportunities for cost-effective prevention of late-life depression: An epidemiological approach. Archives of General Psychiatry $63,290-296$.

Spijker, J. \& Nolen, W.A. (1998). Predictive factors for chronicity of depression: A literature review. Tijdschrift voor Psychiatrie 40, 696-708 [in Dutch].

Spijker, J., De Graaf, R., Bijl, R.V., Beekman, A.T.F., Ormel, J. \& Nolen, W.A. (2002). Duration of major depressive episodes in the general population: results from The Netherlands Mental Health Survey and Incidence Study (NEMESIS). British Journal of Psychiatry 181, 208-213.

Spijker J, Bockting CLH, Meeuwissen JAC, Vliet IM van, Emmelkamp PMG, Hermens MLM, Balkom ALJM van, namens de Werkgroep Multidisciplinaire richtlijnontwikkeling Angststoornissen/ Depressie (2013). Multidisciplinaire richtlijn Depressie (Derde revisie). Richtlijn voor de diagnostiek, behandeling en begeleiding van volwassen patiënten met een depressieve stoornis. Utrecht: Trimbosinstituut.

Trompetter, H.R., Bohlmeijer, E.T., Veehof, M.M. \& Schreurs, K.M.G. (2015). Internet-based guided self-help intervention for chronic pain based on Acceptance and Commitment Therapy: A randomized controlled trial. Journal of Behavioral Medicine 38, 66-80.

Van Vliet, I. M. \& De Beurs, E. (2007). The MINI-International Neuropsychiatric Interview (M.I.N.I.). A brief structured diagnostic psychiatric interview for DSM-IV and ICD-10 psychiatric disorders [in Dutch]. Tijdschrift voor Psychiatrie 49, 393-397.

Zorginstituut Nederland (2014a). Dutch Health Care Insurance Borad [in Dutch] (http://www.medicijnkosten./nl).

Zorginstituut Nederland (2014b). Pharmaceutical compass [in Dutch] (www.farmacotherapeutischkompas.nl).

Zorginstituut Nederland (2015). Kostenhandleiding: Methodologie van kostenonderzoek en referentieprijzen voor economische evaluaties in de gezondheidszorg. Zorginstituut Nederland: Diemen. 


\section{Appendix A}

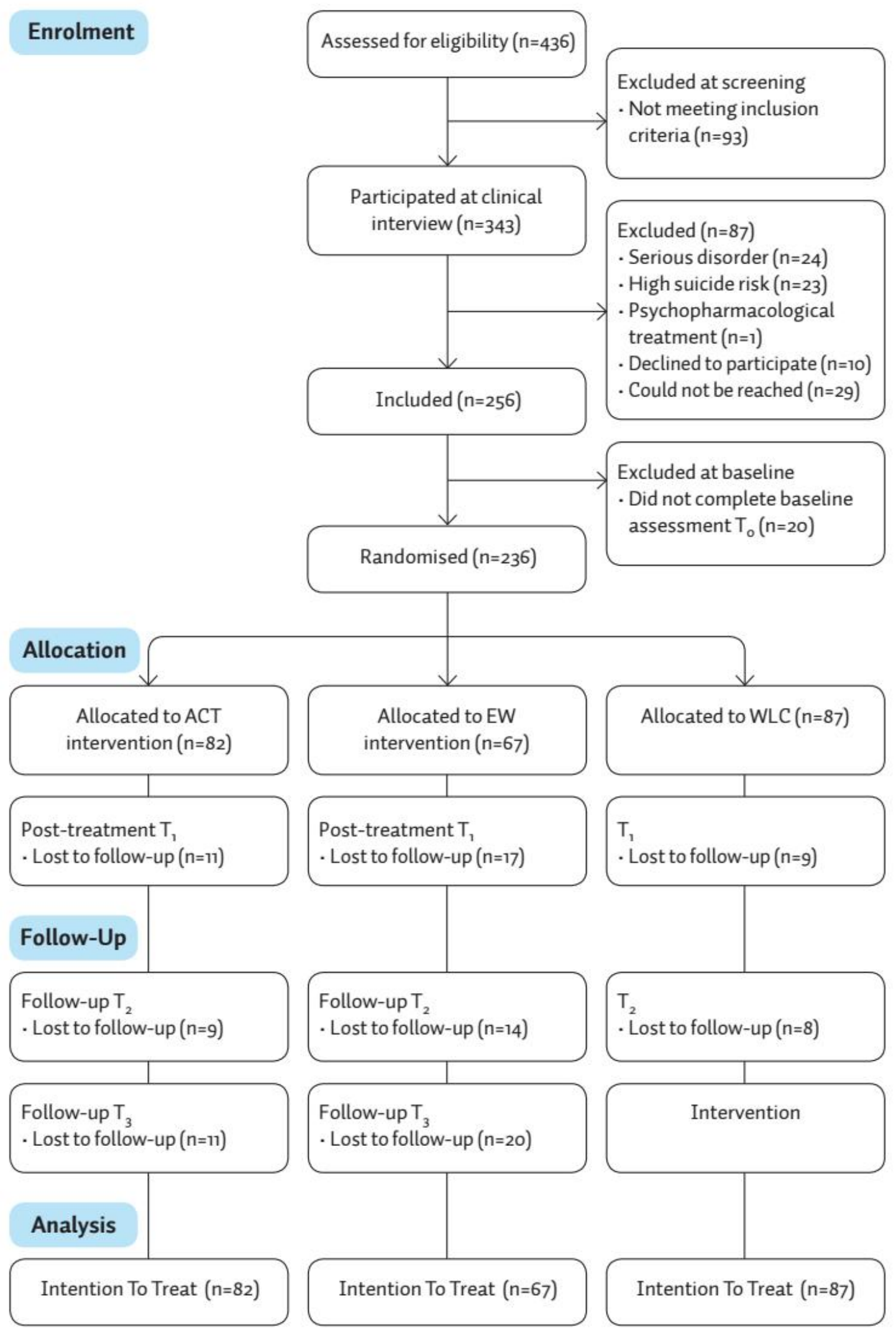

Figure 1 - CONSORT flow of the participants. 


\section{Appendix B}

\section{Calculation of intervention costs}

In the scenario of offering the intervention in community care, participants would have to pay $€ 29.95$ for a licence. In addition, the time spend on the intervention was valued at $€ 12.50$ per hour (i.e. the opportunity cost of leisure time) for an average of 3 and 1.5 hours per week per participant over the nine week intervention period for ACT and EW respectively. The costs of guiding participants through the intervention by the GP's mental health nurse consists of the intake ( $€ 17)$ and evaluation of the screening results ( $€ 17$ ), and $€ g$ for additional contacts by email with a maximum of nine emails. Further costs were related to hosting and maintaining the website at $€ 3000$ per annum. The costs for periodically upgrading the intervention were estimated at $€ 5500$ for the web-based ACT intervention and $€ 1200$ for the web-based EW intervention. Based on data from the central bureau of statistics (Statistics Netherlands) (2013), $10.3 \%$ of the adult population in the Netherlands is having depressive symptoms or a subclinical depression (reference year 2011). It is assumed that about 2 to $3 \%$ of the subpopulation engages in a web-based public mental health intervention (Riper et al., 2013). Assuming a $3 \%$ reach, this would result in an estimated usage by 37,080 participants per year. The per-participant intervention costs were therefore estimated to be $€ 29,95$ (licence) + $€ 337,5$ (hours ACT) or $€ 168,75$ (hours EW) + $€ 115$ (psychologist) $+(€ 3000$ hosting $+€ 5500$ upgrading $/ 37,080$ people $)=€ 483$ for ACT and $€ 314$ for EW. 
CHAPTER 7 


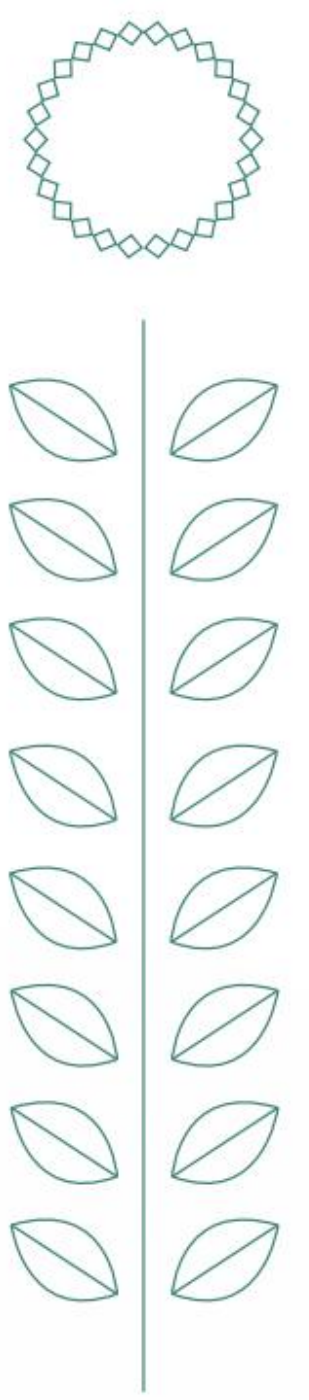

CHAPTER 8 


\section{General discussion}
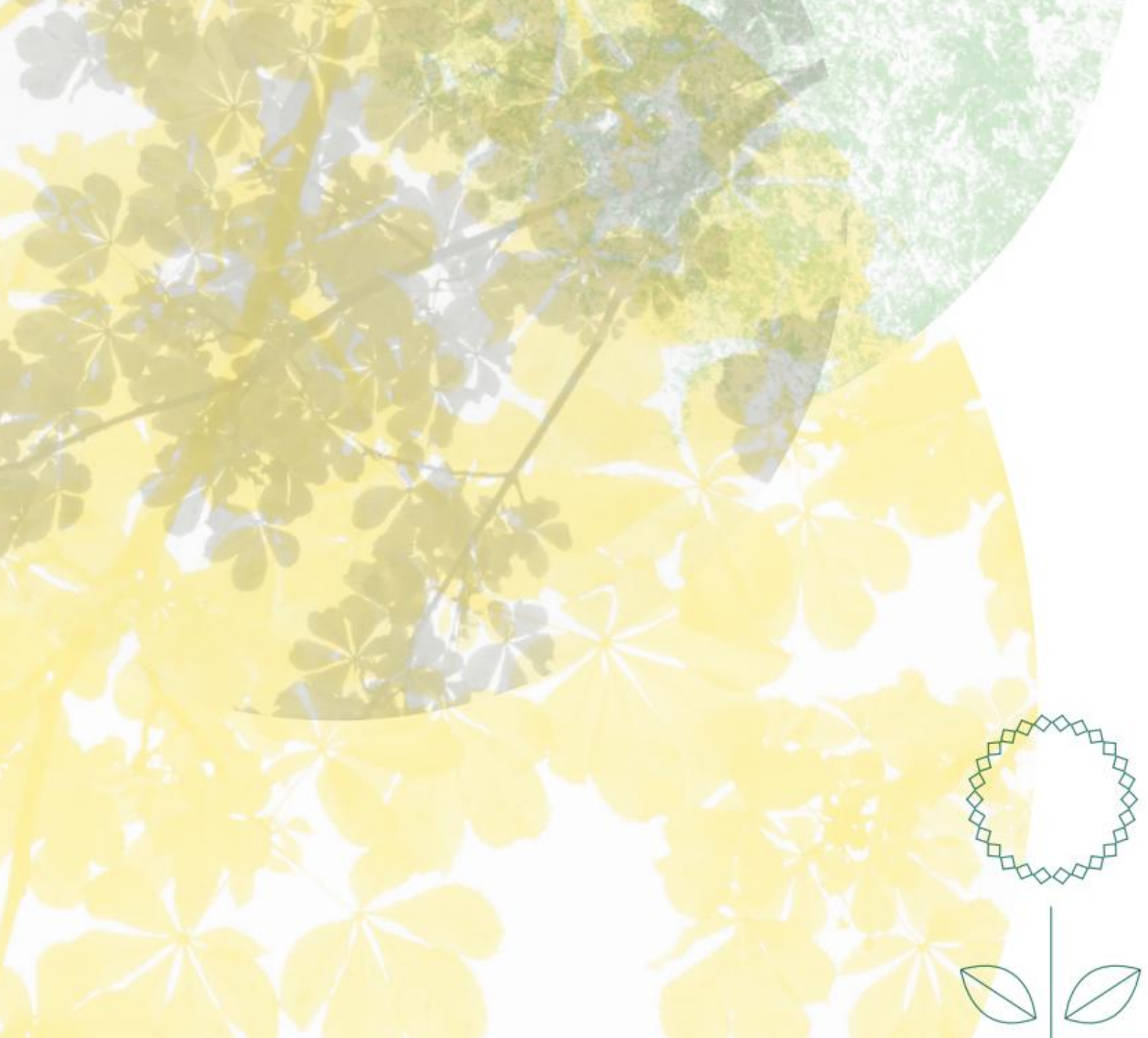


\section{Introduction}

Depressive symptoms are not only a risk factor for developing a depressive disorder, but can also be economically costly and have a considerable impact on one's quality of life. Interventions that are easily available, attractive and feasible help to broaden the effect public mental health interventions can have on depression. Currently, Mindfulness-based Interventions (MBIs) are rapidly making headway as public mental health interventions for people with mild to moderate depressive symptoms. Because research of the effectiveness of MBIs remains limited, it is important to study whether they can be effective public mental health interventions for adults with mild to moderate depressive symptoms. Consequently, we studied a specific subset of MBIs, namely a community-based Mindfulness Based Cognitive Therapy (MBCT) and a web-based Acceptance and Commitment Therapy (ACT) intervention. This chapter starts with a summary of the main results, followed by a discussion of the impact and efficacy of (online) MBIs. Next, we focus on the development and effectiveness of the web-based intervention "Living to the Full," which is based on ACT and mindfulness for adults with mild to moderate depressive symptoms. This discussion is followed by the implications for clinical practice of public mental health interventions in general and the web-based ACT intervention in particular. Finally, we stipulate the limitations of this research and its implications for future research.

\section{General conclusions}

Our first goal was to study whether MBCT as a public mental health intervention could be effective when specifically adapted for people who have depressive symptoms. The adaptation was, in large part, a reduction in mindfulness meditation exercises, from 45 minutes a day as prescribed in the original MBCT to 15 minutes a day. Our study showed that $\mathrm{MBCT}$, originally developed to prevent the relapse of people diagnosed with recurrent depression, can also be effective for people with depressive symptoms.

Although current public mental health interventions have been demonstrated to be effective, they seem to have low impact due to the stigma and a mismatch in acceptability to users. Ensuring availability of interventions on the Internet can enhance the impact of public mental health interventions and improve the quality of health care. Our second goal was to study whether web-based or online public mental health interventions based on mindfulness were effective. In our meta-analysis, online MBIs as public mental health interventions showed a beneficial impact on mental health outcomes, suggesting that online MBIs have the potential to contribute to promoting mental health. 
Our third goal was to broaden the availability of online MBIs in a (cost-)effective way by developing a web-based intervention "Living to the Full" for adults with mild to moderate depressive symptoms. We developed the web-based intervention "Living to the Full' to match the values of the stakeholders, using persuasive technologies to increase adherence. The web-based ACT intervention proved to be superior over time in effectiveness compared to both the active and the waiting list control group. Psychological flexibility and mindfulness appeared to be the central therapeutic mechanisms in the web-based ACT, confirming its theoretical framework. Furthermore, we examined the demographic characteristics, psychological symptoms, diagnostic classification and comorbidity of our target group, and, at least for similar groups, there seems to be no reason to exclude people from web-based ACT.

In conclusion, our findings contribute to the current research and set the stage for MBIs as public mental health interventions for people with mild to moderate depressive symptoms. From a clinical perspective, our findings suggest that it is useful to implement MBIs in community mental health care. Specifically, web-based ACT can be easily implemented with lower intervention costs as it is a (cost-)effective intervention, at least for women with mild to moderate depressive symptoms and a high educational level.

\section{MBIs as public mental health interventions}

MBIs have the potential to offer effective and acceptable treatments by means of prevention or early intervention that transcend traditional health care. There is now growing recognition that mental health care should not only focus on reducing psychological symptoms, but also should promote positive mental health (Slade, 2010). This kind of approach is in line with the focus of the two-continua model of Keyes (1995) that states that mental illness and well-being are distinct but related phenomena. Keyes' perspective has been integrated in the new era of public mental health interventions that are based on mental health promotion (Bohlmeijer et al., 2011; Bolier et al., 2013b; Jané-Llopis et al., 2005; Rashid, 2009).

The use of MBCT as a mindfulness-based public mental health intervention has been recently on the rise. Mindfulness is a trending topic nowadays, appearing on the cover of TIME magazine in 2014, and being regularly offered by community mental health care centres. Although mindfulness research has exponentially increased during the last couple of years (see Figure 1; American Mindfulness Research Association, 2016) and studies continue to show its potential, empirical data on MBIs as a public mental health intervention is still lacking. Mindfulness, in the context of MBCT, was originally designed to prevent relapse in people diagnosed with recurrent depression 


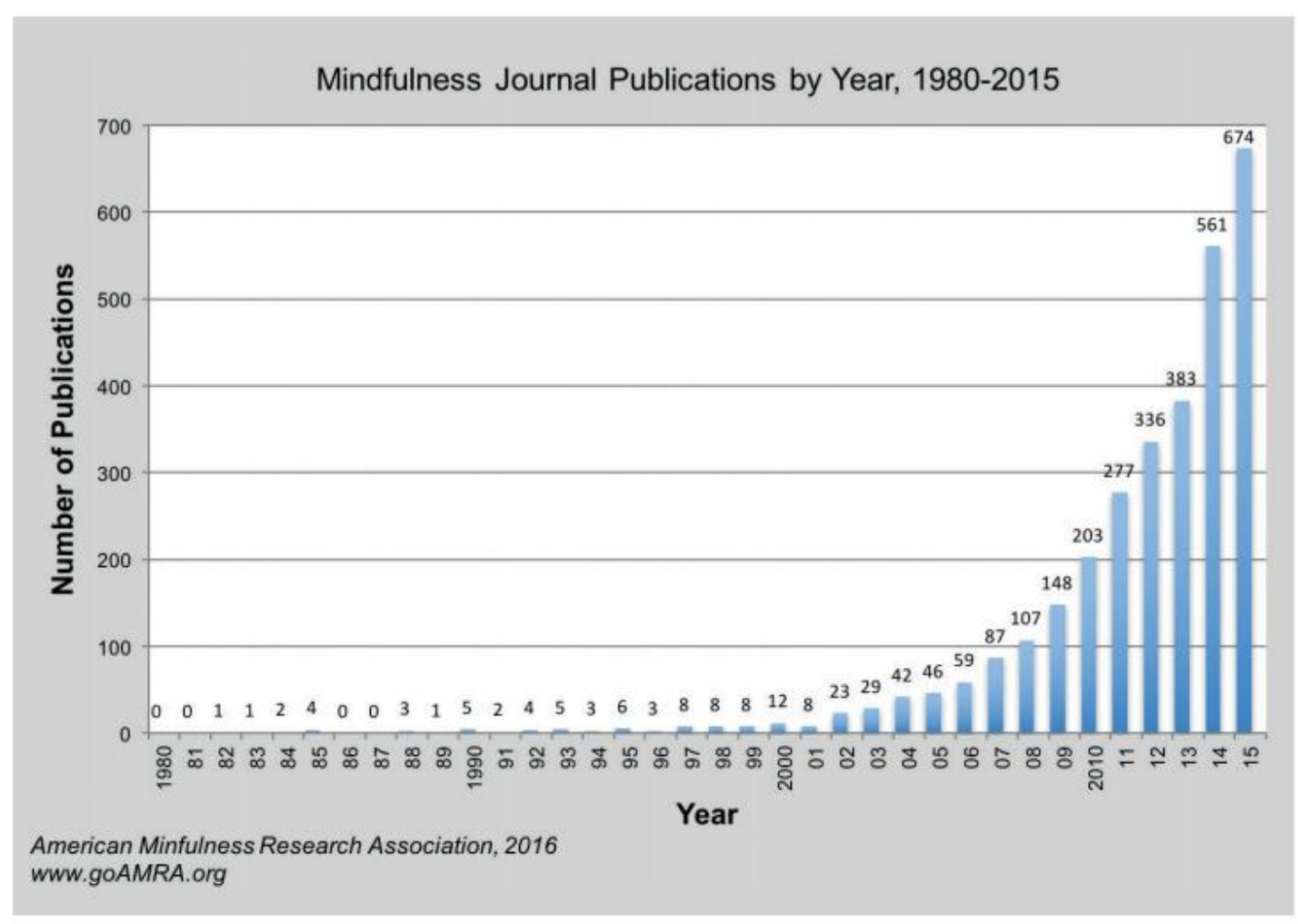

Figure 1 - Mindfulness journal publications from 1980 to 2015. (American Mindfulness Research Association, 2016).

(Ma and Teasdale, 2004; Segal et al., 2002; Teasdale et al., 2002; Teasdale et al., 2000). In Chapter 2, we showed that MBCT as a public mental health intervention with brief mindfulness exercises can also be effective for people with depressive symptomatology. This finding corresponds with recent meta-analyses on MBIs that indicated that MBCT can have a beneficial effect beyond the treatment of MDD in remission (e.g., Hofmann et al., 2010; Strauss et al., 2014). Our study of a community-based $\mathrm{MBCT}$, as described in Chapter 2, contributed to this research, as it demonstrated the effectiveness of $\mathrm{MBCT}$ as a public mental health intervention for people with depressive symptomatology. Furthermore, our study showed that a low intensity community-based MBCT can be effective, compared to a waiting list control group, with an effect size of $d=0.50$ post-treatment and $d=0.40$ at 6 -months follow-up. These findings are in line with Khoury et al. (2013) who performed a meta-analysis of 209 studies on MBIs for a wide range of conditions and disorders, including non-clinical populations. Their results showed that MBIs had moderate effect sizes pre-post (0.55), and small to moderate compared to waiting list and active controls (0.53; 0.33), which are comparable to traditional CBT or behavioural therapies. Furthermore, Cavanaugh et al. (2014) showed in their meta-analysis that mindfulness and acceptance as self-help interventions have a beneficial potential for depressive 
symptoms with small to moderate effect sizes ( $g=-0.37,95 \%$ CI -0.19 to -0.56$)$. They defined self-help interventions as low intensity interventions, requiring no guidance or reduced guidance, with an aim to instruct, guide and encourage the user to develop skills, manage their difficulties and make changes, rather than just providing information. Our community-based MBCT was not a self-help intervention as it was offered as a group course, but it was low intensity in the fact that the original high impact exercises of 45 minutes a day were reduced to daily 15 minutes practices. In line with our study on community-based $\mathrm{MBCT}$, recent studies have suggested that brief mindfulness exercises of 15-30 minutes are also effective (Carmody and Baer, 2009). This is an important finding, because to broaden the impact of public mental health interventions it is crucial to make interventions attractive and feasible. A lot of people with subclinical symptoms are employed and socially active, and, therefore, investing longer periods of time may be a barrier to their ability or willingness to participate in an intervention (Carmody and Baer, 2009). The availability of a low intensity community-based MBCT can further expand the impact of public mental health for adults with depressive symptoms.

\section{Mechanisms of change}

The essential question is whether mechanisms of change from the perspective of MBIs hold true for public mental health interventions since such interventions are usually adjusted in format to low intensity and/or web-based. For MBCT, research has shown that the development of mindfulness skills stimulates the cultivation of meta-awareness (or decentering), helping the individual to ultimately disengage from rumination, a common activity during depression (Segal et al., 2002; Teasdale et al., 1995). As described in Chapter 2, mediational analyses on our communitybased $\mathrm{MBCT}$ intervention confirmed that mindfulness skills are important mediators of change in the community-based MBCT. Furthermore, psychological flexibility was found as a mediator of the effects on depressive symptomatology. This is in line with earlier studies that demonstrated the association between psychological inflexibility and psychopathology (Hayes et al., 2006; Kashdan and Rottenberg, 2010). This increase of psychological flexibility and mindfulness mediating the effects of MBCT on depressive symptomatology suggests that participants have gained additional adaptive emotion regulation skills in response to negative affect-producing stressors (Chiesa et al., 2014; Kashdan and Rottenberg, 2010).

For ACT, our study on the mechanisms of a web-based ACT in Chapter 6 corroborates with the evidence that psychological flexibility and mindfulness are distinct process mechanisms that mediate the effect of web-based ACT on depressive 
symptoms. Furthermore, our research strengthened the relevance of psychological flexibility as the theoretical framework of ACT (e.g., Forman et al., 2012; Hayes et al., 2006; Kashdan and Rottenberg, 2010; Niles et al., 2014; Wicksell et al., 2011). Although our study did not use the prerequisite of temporal mediation, our results are consistent with Fledderus et al. (2013) who performed a mediation study using cross-lagged regressions, concluding that effects on depression by an ACT intervention were mediated by improvements in psychological flexibility during the intervention.

Together, psychological flexibility and mindfulness may reduce engaging in maladaptive emotion-regulation strategies that set the stage for negative emotional experiences that can persist into depression. However, research has also shown other important mechanisms of change in depressive disorders, such as rumination, worry and neuroticism (e.g., Nolen-Hoeksema, 2000; Olatunji et al., 2013; Spinhoven et al., 2016). Spinhoven et al. (2016) performed a longitudinal cohort study in a large sample of 2981 adults, including those with lifetime and/or current depressive disorder as well as healthy controls. They found that experiential avoidance moderately to strongly correlated with rumination, worry and neuroticism, suggesting a common etiologic factor shared among psychological vulnerabilities for depression. The authors argued that their results indicated that:

\footnotetext{
... experiential avoidance and bordering psychological vulnerabilities are highly associated and probably share a tendency to frequently experience strong negative emotions, to evaluate these experiences as aversive and to engage in avoidant coping strategies. This tendency may represent a transdiagnostic risk factor traversing a broad range of depressive and anxiety disorders (p. 161).
}

This perspective is in line with the concept of psychological flexibility as a unified model of human functioning (Hayes et al., 2012), and the broader context of emotion regulation as a transdiagnostic risk factor for depression and emotion disorders (Aldao and Nolen-Hoeksema, 2010; Hofmann et al., 2012; Norton and Paulus, 2015).

In conclusion, within the research to date, psychological flexibility and mindfulness skills seem to be important mechanisms of change in MBIs (e.g., Chiesa et al., 2013; Farb et al., 2012; Hölzel et al., 2011; Kuyken et al., 2010). As such, public mental health interventions based on mindfulness or ACT have the potential to increase psychological flexibility and mindfulness, thereby reducing the risk for developing depression and possibly other disorders as well. However, further examination of processes of MBIs in the context of depression are needed. 


\section{Entering a new era: online MBIs}

Online interventions are easily accessible, scalable and may help to tackle some of the limitations of traditional treatments (Andersson and Titov, 2014; Cuijpers et al., 2010). Hence, online MBIs are becoming part of the new era of public mental health. The advantages of online interventions are apparent, as the dissemination of available and effective treatments for depression is still lacking, and health care services are facing long waiting lists (Andrews et al., 2004; Shafran et al., 2009). The meta-analysis on online MBIs in Chapter 3 transcends the research so far by examining the effectiveness of online MBIs on mental health outcomes. To our knowledge, our study was the first to examine the overall effectiveness of online MBIs on depression, anxiety, stress, well-being and mindfulness. The meta-analysis included 15 studies, with a total of 2360 participants, in clinical and non-clinical populations. The results showed that MBIs have a significant small to moderate ( $g=0.22$ to 0.51 ) beneficial impact as public mental health interventions, suggesting that MBIs have the potential to contribute to promoting mental health. Particularly interesting in this study, a small effect was found $(g=0.29)$ for depression, with a moderate level of heterogeneity and no substantial effect of outliers or the quality of studies on the effect size. This suggests that, even with the limited studies to date, MBIs can effectively be delivered online for people with depressive symptoms. Contrary to the literature, however, the effect size of online MBIs was smaller than the moderate to large effect sizes in traditional face-to-face MBIs (Abbott et al., 2014; Cavanagh et al., 2014; Hofmann et al., 2010; Khoury et al., 2015; Piet and Hougaard, 2011; Vøllestad et al., 2012; Zainal et al., 2013). As the number of the included studies in our meta-analysis were small and additional analyses were exploratory, the effect size must be interpreted with caution and further research is warranted. Another possible explanation for the lower effect sizes of online MBIs for depression is non-adherence, which occurs when participants stop following the intervention protocol. Non-adherence is a regular finding with regard to the smaller effects reported in online interventions (Christensen et al., 2009; Eysenbach, 2005; Kelders et al., 2012). Adherence could be especially relevant in $\mathrm{MBIs}$, as regular practice is assumed to be necessary for developing mindfulness skills (e.g., Carmody and Baer, 2008). In our meta-analysis, adherence varied between $35 \%$ and $92 \%$ when defined as completion of all sessions ( $n=5$ studies), but definitions of adherence varied among the studies, making subgroup analyses impossible. For future research, it would be interesting to more specifically explore adherence rates of online MBIs, as well as the definitions of adherence.

A recent concern raised in mindfulness research is the concept of positive publication bias, which is the over-publication of positive results on trials (CoronadoMontoya et al., 2016). In their meta-analysis, Coronado-Montoya et al. (2016) found 
that there were 1.6 times more positive results reported as would be expected, and that of the 124 randomised controlled trials (RCTs), only 21 were registered prior to data collection. Only studies with clinical populations (as opposed to general populations, students, and employees) and studies that required mental health symptoms for enrolment were consistent. This is, however, not a new phenomenon nor is it solely found within the mindfulness research literature. Coronado-Montoya and colleagues' results are consistent with earlier findings on the effectiveness of antidepressants (Turner et al., 2008) and CBT (Driessen et al., 2015), confirming that positive publication bias can be a problem and calling for improved standards for research methodology and publication. As the field of mindfulness research is rapidly progressing and the meta-analysis of Coronado-Montaya included studies only up until July 2013, new research complying with the recommended standards are now more widely available (e.g., Carlson et al., 2013; Hoge et al., 2013; Kuyken et al., 2015; Kuyken et al., 2016; Pots et al., 2015; Trompetter et al., 2014), making headway for the effectiveness of online MBIs.

\section{Developing for adherence}

Non-adherence (i.e., when a participant does not follow the intervention protocol) is common in web-based interventions and is called one of the main reasons for low impact of web-based interventions (Christensen et al., 2009; Donkin et al., 2011; Eysenbach, 2005; Kelders et al., 2012). As such, it is imperative to address non-adherence as early as possible when designing an intervention. To reduce the impact of non-adherence in web-based interventions, it is also essential to develop interventions that best fit the users. Kelders and colleagues (2012) have shown that intervention characteristics as well as the persuasive design of web-based interventions have an effect on adherence. Persuasive technology refers to the capacity of technology to influence behaviour and is used in eHealth research to understand the role of technology in changing behaviour (Fogg, 2003; Oinas-Kukkonen and Harjumaa, 2009). Web-based interventions are usually developed by deducing the content of psychological protocols and by taking technology and the human context as a fait accompli. This ad-hoc design seems to be one of the main reasons that web-based interventions do not reach their full potential in terms of adherence and outcome (Pagliari, 2007; van Gemert-Pijnen et al., 2011). The Center for eHealth Research and Disease Management (CeHRes) Roadmap presents a framework for the design of a web-based intervention that applies a holistic approach that includes persuasive technology theories, human-centered design, and business modelling (van Gemert-Pijnen et al., 2011). Usage of this framework results in an intervention that benefits from collabo- 
rative knowledge and expertise, matching the values of the different stakeholders. In Chapter 4, we presented the results of the development of the web-based ACT intervention "Living to the Full", for which we employed the CeHRes Roadmap to achieve a user-friendly application that fit the values of the stakeholders in an iterative process. Using the framework of the Persuasive System Design-model (PSD; Oinas-Kukkonen and Harjumaa, 2009), we implemented persuasive technology into the design of the web-based ACT intervention. The PSD-model classifies technology in its persuasive functions: primary task support, dialogue support, social support, and credibility support. Persuasive technologies were implemented by using text messages tailored to the content of the module, tailored stories or motivation, multimedia messages, interactive exercises, and an option to personalise the homepage. In a parallel study of the same web-based ACT intervention, using a fractional factorial randomised controlled design, the effectiveness of all the persuasive features was studied (Kelders et al., 2015). In this parallel study, the web-based ACT intervention with automated support was compared to the same intervention with human support. The results showed that automated support can be as effective at 6-months follow-up as human support, which confirms the great potential of a web-based ACT intervention in public mental health or community care. All other persuasive features showed no difference on outcome or adherence, which does not align with other studies (Fogg, 2003; Fry and Neff, 2009; Hurling et al., 2006; Noar et al., 2007; Oinas-Kukkonen and Harjumaa, 2009; Ritterband et al., 2006; Strecher et al., 2008; Webb et al., 2010). A possible explanation could be that there was too little variation between the different features or components of the intervention (Kelders et al., 2015), or that the usage of all the components was not optimal (Kelders et al., 2013). As research on persuasive features is relatively new (Kelders, 2012; Oinas-Kukkonen, 2010), more studies of adherence are needed that explore its relationship to the effectiveness of the interventions (see e.g., Wildeboer et al., 2016).

Overall, we found that by developing the technology in collaboration with stakeholders, we successfully redesigned the system, content and service of the web-based ACT intervention to match the values of the stakeholders. The usage of a human-centered design, which advocates the systematic, continuous consultation of potential users during the whole process, has been shown in other research to have a positive effect on user satisfaction and on fitting users' needs (Gould and Lewis, 1985; Kujala, 2003). 


\section{Impact of the web-based ACT intervention}

Our aim was to broaden the availability of online MBIs in a (cost-)effective way by developing a web-based intervention "Living to the Full", which is based on ACT and mindfulness, for adults with mild to moderate depressive symptoms. To study the impact of such a web-based ACT intervention, a large randomised controlled trial was performed with a comparison of two control groups.

\section{Effectiveness}

To our knowledge, this study is the first to investigate a web-based ACT self-help intervention for adults with depressive symptoms or mild depressive disorder. One strength of the study, as discussed in Chapter 5 , is that the ACT intervention was not only compared to a waiting-list control (WLC) group but also to an active control condition in the form of a web-based Expressive Writing (EW) intervention. The study design of a RCT is largely debated, and comparisons with a waiting list condition alone are expected to reveal larger effect sizes than designs that include an active control condition (Cuijpers et al., 2008b; Mohr et al., 2009), as is shown by several meta-analyses (Andersson and Cuijpers, 2009; Andrews et al., 2010; Cavanagh et al., 2014; Grist and Cavanagh, 2013). Our study indicated that ACT over time (o to 6 months) was significantly more effective than both control conditions. At 3 months after baseline, effect sizes for depression were small to moderate (Cohen's $d=0.56$ for ACT versus WLC and $d=0.36$ for ACT versus EW), but at six and twelve month follow-up both interventions had similar effects on depressive symptoms. The small to moderate effect sizes post-treatment are largely consistent with recent meta-analyses of web-based interventions for depression (Andersson and Cuijpers, 2009; Andrews et al., 2010; Arnberg et al., 2014; Baumeister et al., 2014; Richards and Richardson, 2012). These effect sizes also correspond to the effects of psychotherapy for depression as discussed in the research literature in general (Cuijpers et al., 2008a), as well as meta-analyses on ACT as self-help and as face-to-face treatment (A-Tjak et al., 2015; Gu et al., 2015; Hacker et al., 2016; Öst, 2014; Ruiz, 2010). Furthermore, our study showed that the proportion of participants in the ACT intervention who made a clinically significant change in depressive symptoms was significantly higher than in both control conditions at post-treatment; $54 \%$ against $26 \%$ in WLC and $31 \%$ in EW. These results demarcate the web-based ACT intervention as superior to EW or WLC at post-treatment for adults with mild to moderate depression. The results from the regression analyses on diagnostic classification showed a positive trend, but all were insignificant. The only significant proportional improvement was for the diagnosis of recurrent depression for both the ACT and the EW intervention as compared to the WLC. As the presence of clinically relevant depressive symptoms 
is known to be a risk factor for clinical depression (Cuijpers and Smit, 2004), this outcome could suggest that the web-based intervention has potential to decrease the risk of a new episode of depression. Moreover, Geschwind et al. (2012) in their study advocated that residual symptoms have a sizable impact on quality of life and represent a major risk factor for depressive relapse of recurrence. Furthermore, ours is the first study to show that the effects of the web-based ACT intervention were maintained at 12-month follow-up. The maintenance of the effect is similar to findings in earlier studies that examined the effect of ACT on depression (Bohlmeijer et al., 2011; Fledderus et al., 2012).

In Chapter 6, we described our study, which explored for whom and under what circumstances the web-based ACT intervention had differential effects. This area of research is important, as it is assumed that face-to-face treatments are not comparable to web-based treatments. The latter place a greater burden on self-efficacy and resilience, and have high non-adherence rates. If we can differentiate for whom the web-based ACT intervention works best, we can help tailor interventions for different sub-populations and ultimately improve clinical decisions regarding treatment. The moderator analyses demonstrated that the effects of the web-based ACT interventions did not vary according to baseline patient characteristics when compared to both control groups. These results indicated that there are no restrictions to the allocation of a web-based ACT intervention, at least not for the studied target group (mostly women with depressive symptoms and a middle to high educational level). This is a significant finding as web-based interventions can have a large incremental impact in availability and accessibility of interventions (Andersson and Titov, 2014; Andrews et al., 2004), and, consequently, on the incidence of MDD.

We described a health-economic evaluation alongside our RCT in Chapter 7, to evaluate if the web-based ACT intervention would lead to increased health gains at lower costs. Overall, at 6 months, the ACT intervention was only successful compared to $\mathrm{EW}$ in reducing depressive symptoms and increasing quality of life, being the dominant intervention. This effect recedes at 12 months, where ACT is no longer significantly more effective than EW. Compared to the WLC, the ACT intervention was not significantly more effective in increasing health gains at 6 months. However, with lower intervention costs, sensitivity analyses showed that the web-based ACT intervention was the dominant intervention compared to both control conditions. These latter results are important as the overall expenditure for health care is increasing. The web-based ACT intervention is at present in the Netherlands implemented in community care with automated support, thus lowering the costs of offering the ACT intervention. Pro-actively offering web-based ACT could be a feasible solution, since web-based ACT generates clinical effectiveness at an earlier point in time ( 3 months), is scalable and would lower the per-patient health care costs. 
To our knowledge, this thesis presents the first economic evaluation of a webbased ACT intervention for depression. In line with recent meta-analyses showing that ACT can be effective for depression (A-Tjak et al., 2015, Hacker et al., 2016, Öst, 2014, Ruiz, 2010), our clinical trial, described in Chapter 5, showed that ACT was over time more effective than both control conditions (Pots et al., 2015). However, this economic evaluation indicates that the web-based ACT intervention is only cost-effective from a societal point of view compared to the EW intervention. Costeffectiveness studies on Internet-based Cognitive Behaviour Therapy (ICBT) for depression (Arnberg et al., 2014), and mental disorders (Donker et al., 2015; Hedman et al., 2012; Mihalopoulos and Chatterton, 2015) show that ICBT can have a 50\% probability of being more cost-effective compared to no treatment or to conventional CBT when willingness to pay for an additional improvement was zero. This compares well with the results in our study, at least for the comparison with the active control group ( $56 \%$ probability). This is an important finding, as the presence of clinically relevant depressive symptoms is known to be a risk factor for clinical depression (Cuijpers and Smit, 2004), the results of our study could suggest that the web-based ACT intervention has potential to decrease the risk of a new episode of depression sooner than both control conditions. However, these results are exploratory and need further research, especially with regard to the longer-term cost-effectiveness.

\section{Mental health promotion}

The web-based ACT intervention not only resulted in superior post-treatment effectiveness by decreasing depressive symptoms, but also in increasing positive mental health. We measured positive mental health with the Mental Health Continuum-Short Form (MHC-SF), and found that the web-based ACT intervention was significantly more effective from baseline to post-intervention compared to both the EW intervention and the WLC, and this effect was maintained at follow-up. Looking at the different dimensions of positive mental health (psychological, emotional and social well-being), we noted that psychological well-being was significantly improved post-treatment in the ACT intervention compared to both control groups. The other two dimensions - emotional well-being and social well-being - only showed significant results for ACT versus WLC. This finding indicates that web-based ACT is particularly effective in improving psychological well-being, i.e. optimal psychological functioning and meaning in individuals' lives by increasing (among others) self-acceptance, autonomy, positive relationships with others, and purpose in life. The results confirm previous research that showed that ACT interventions are effective in promoting positive well-being (Fledderus et al., 2012; Forman et al., 2007; Kashdan and Ciarrochi, 2013; Lappalainen et al., 2007). The finding that ACT increases positive mental health might be particularly relevant, as growing research demonstrates that 
having positive mental health protects against the risk of mental illnesses (Keyes et al., 2010; Lamers et al., 2015; Wood and Joseph, 2010). Also, Ryff (2014) reviewed a large number of studies and found that psychological well-being is related to a large variety of positive mental and physical health indicators. From the perspective of depressed patients, Zimmerman et al. (2006) performed a qualitative study, showing that aspects of positive mental health, such as self-confidence and a return to a usual level of functioning, were judged to be more important than symptom resolution. This underscores the importance of the availability of public mental health interventions from a (positive) mental health promotion perspective.

\section{Adherence in web-based ACT}

The web-based ACT intervention was developed to achieve a user-friendly application that fitted the values of the stakeholders and to increase adherence by implementing persuasive technology. Adherence in our study was indeed higher than regularly found in other studies. Both the web-based ACT and the EW intervention had high adherence rates of $84 \%$ and $78 \%$ respectively. This implies that we successfully developed an intervention that decreased non-adherence and thereby potentially increased the effect of the intervention. To insure the effectiveness of an intervention, it is imperative for the designers to understand the use of content and persuasive features. By using log data (data collected by the computer) insights can be gained into the usage and use patterns of participants. In the parallel trial on web-based ACT mentioned earlier, Kelders et al. (2013) concluded that adherers showed more log-ons per lesson, downloaded more mindfulness exercises, and viewed more text messages than nonadherers, confirming that adherers show more involvement with the intervention. This higher engagement within a lesson may be beneficial for the effectiveness of the intervention because more exposure to a web-based intervention has been shown to increase its effectiveness (Donkin et al., 2011). The log data further showed that most of the participants who did not adhere to the intervention, started to non-adhere during the first three lessons ( $55 \%, 48$ out of 88 participants started the first lesson, but did not adhere to the intervention). Notably a fairly large group of participants also started to non-adhere during Lesson 6. The web-based ACT intervention consisted of nine online lessons, to be completed one per week. The nine lessons were divided into three parts, which reflect the six core processes of ACT. Interestingly, the start and end of these parts can be deemed confronting, which can lead to nonadherence or - as intended - to committed action. Kelders et al. (2013) suggested that these moments of non-adherence could be eased by deploying persuasive technology, for instance, by providing participants more interaction with the system as it acknowledges how most people tend to struggle during this phase. Mohr et al. (2011) proposed that human support may increase adherence at these times by 
providing the right amount of support (tailored to the individual). In line with this suggestion, Hadijstavropoulos (2016) found that it may be helpful for depressed patients to receive more text messages mid-treatment to bolster the therapeutic alliance. Overall, for a web-based intervention to be effective, it is not only imperative to have a good randomised controlled design, but also to design for adherence and to understand more about the usage of the content and features.

\section{Implications for clinical practice}

Public mental health interventions are a complementary strategy to the treatment of depression in in- and out-patient settings. By focusing on pro-actively offering public mental health interventions for people that have depressive symptoms, but not MDD, more people can be protected from developing MDD (Muñoz et al., 2010). Therefore, making public mental health interventions available can curtail the burden of disease. Using a positive framework and promoting positive mental health as well as reducing psychological distress can increase the opportunity of reaching more people. Public mental health interventions need to be easily accessible and available in order to have a considerable impact on health care. International interest is continually growing in the potential of digital solutions to enhance the availability and quality of public mental health interventions. As a result, we are entering a new era of public mental health interventions, and web-based interventions are increasing, raising the question of how best to implement public mental health interventions, and specifically web-based interventions. Based on the results of this thesis, one could decide to take a practical approach and implement web-based public mental health interventions on a large scale. However, from a clinician perspective, some concerns with this approach remain outstanding.

\section{Implementing public mental health interventions}

The way to implement public mental health interventions is perhaps best answered in the context of stepped-care (e.g., Bower and Gilbody, 2005; Gilbody et al., 2006; Van Den Broeck et al., 2016). In The Netherlands, the health care system is organised stepped-care, from community care to primary care and secondary care, in line with guidelines for evidence-based treatment (Mental Health Guidelines, www.ggzrichtlijnen.nl). In the revised guidelines, web-based interventions (bibliotherapy or guided web-based interventions) are now standardised parts of first-step interventions, parallel to watchful waiting and psycho-education (Spijker et al., 2013). Examples are "Psyfit” (Bolier et al., 2013a), "Kleur je leven" ("Colour Your Life”; De Graaf et al., 2009, 2011), "Grip op je dip" ("Master Your Mood”; Van Der Zanden et al., 
2012), and "Geen Paniek" ("Don't Panic"; Meulenbeek et al., 2010). In addition, the web-based ACT intervention has been made available with lower intervention costs since 2015 within community health care, with automated support. The advantages are apparent, as these public mental health interventions are widely available and more cost-effective than regular care because they require little or no human support. As a result, there is a growing interest in, and adoption of, web-based interventions in The Netherlands. In 2010, almost 1.8 million people visited an online help site for psychiatric or social problems, two out of three Dutch mental health care institutions apply eHealth in their care provision, between 60 and $70 \%$ of mental health care clients with a diversity of complaints are receptive to the idea of eHealth, and the number of people receiving Internet-based help for depression has tripled over a period of three years (Riper et al., 2013). This implies the need for eHealth interventions beyond community care.

A recent development in primary and secondary care is the concept of blended care. In blended care, face-to-face and eHealth sessions are interspersed. For example, regular therapy is interlaced with online chat, computer phone calls, eHealth modules and/or parts thereof. Although blended care is not yet part of the guidelines for the treatment of depression, it is a huge step in the direction of easily accessible and effective public mental health interventions. Blended care is expected to be the solution to scaling eHealth (Postel et al., 2015; Riper et al., 2013; Ruwaard and Kok, 2015), but its research is still in its infancy. Further studies on the effectiveness of blended care are imminent (e.g., Kemmeren et al., 2016; Kooistra et al., 2014).

\section{Clinicians approach}

Implementation of eHealth is still insufficient, partly because clinicians are skeptical about the effect eHealth interventions might have on the therapeutic alliance and, therefore, are often reluctant to adopt eHealth for their patients (Postel et al., 2015, Riper et al., 2013). Moreover, clinicians do not seem to understand how online treatments might support face-to-face treatments (van der Vaart et al., 2014; Wentzel et al., 2016). Recent studies have shown that web-based interventions can however be equally effective as face-to-face treatments, while not harming the therapeutic alliance (Andersson et al., 2012; Hadjistavropoulos et al., 2016; Preschl et al., 2011). These studies are in line with the results from this thesis and the parallel study of our web-based ACT intervention that showed at follow-up that automated support was as effective as human support (Kelders et al., 2015). Several other interventions have also found automated support to be effective (Aardoom et al., 2016; Berger et al., 2011; Mohr et al., 2013; Rheker et al., 2015; Tate et al., 2006).

Despite the apparent need for eHealth interventions, clinicians remain unreceptive to the idea of eHealth, indicating the need for use training to change the perspective 
of clinicians and to subsequently help them to find a good fit between the patient and eHealth. Training in eHealth applications is a useful way to help clinicians obtain a broader perspective. Since blended care is not yet a standard guided practice, clinicians still struggle with how to implement eHealth in primary or secondary care. Hence, guidance and training can help clinicians to develop ideas on how to use eHealth as blended care. Examples of blended care are when clinicians integrate eHealth homework assignments or values work for their patients with regular care, or work with a protocol for blended care. One useful approach in facilitating the process of setting up a personalised blended treatment is the Fit for Blended Care instrument (Wentzel et al., 2016). This instrument can help clinicians and patients in a shared decision-making process, and aid clinicians to align existing treatments, or possibly blended care, with the needs of their patients.

Although web-based public mental health appears to be effective, it is not a panacea for all forms of depression. Also, as many depressive episodes remit spontaneously, not all depressed individuals necessarily need prompt treatment (Spijker and Nolen, 1998; Spijker et al., 2002). For now, the most reasonable choice seems to be shared decision making by continuing to ensure the therapeutic alliance, in line with a user-centered approach, and to explore the possibilities of low intensity or web-based public mental health interventions.

\section{Limitations and implications for future research}

The research presented in this thesis has several limitations, which give rise to a number of suggestions for future research. Regarding the outcomes of our studies, generalising results can be problematic. The issue of self-selection bias (i.e. selfreferral and motivation for time-investment) was interwoven into the recruitment strategy of our RCTs. We chose to frame the studies by recruiting participants with mild to moderate depressive symptoms for people from the general population. Our study populations largely consisted of highly educated women. It is a common finding that they are prone to apply for guided web-based self-help interventions (Eysenbach, 2005). Further research should determine the efficacy of web-based interventions for individuals with a lower level of education.

The research on mindfulness is quickly progressing in both quantity and quality. Our meta-analysis on online MBIs was the first study to address the overall impact of online MBIs, but the number of studies included was small and the variability in the studies was large. These factors restricted the statistical power of our subgroup analyses, making our conclusions tentative. Future research on (online) MBIs as public mental health interventions is warranted as the field progresses at a rapid 
pace and MBIs seem a promising strategy in mental health promotion. In addition, studies that specifically address adherence and non-adherence are critical to gain insights into the usage of MBIs. Similarly, by monitoring participants intensely over time, single case experimental designs could provide further insight into usage and use patterns.

Even as interventions become more accessible and effective, eHealth still remains a 'black box' as to what works for whom (Kelders, 2012). The findings from our mediation and moderation study, unfortunately, have not shed much light into or onto the black box. Although the results of our study were encouraging with regard to the broad allocation of web-based ACT for at least the self-selected target group at hand (mainly women with a middle to high educational level), the current research on moderators and predictors is sparse. Mediation studies could also profit from well-powered analyses and better designs, such as cross-lagged panel designs. As the evidence base for online MBIs develops, further analyses of mediators, moderators and predictors of treatment should be explored to help tailor interventions to different subpopulations and improve clinical decisions about treatment.

Our RCT on community-based MBCT was our first study in this thesis, and the design included a waiting-list control. Active control designs are preferred, as they control for the influence of possible non-specific factors, such as attention and social interaction. In our second RCT, we did use a three-armed trial with a waiting-list control group and an active control group. Furthermore, also the follow-up period of three months was relatively short in our first RCT. Short follow-ups are often an ethical choice, at the advantage of the waiting-list group. However, longer follow-ups are suggested to study the impact of a public mental health intervention on the incidence of depression. For future research, longer follow-ups in addition to a threearmed trial are recommended to increase the chance for participants to receive a treatment.

In our study on web-based ACT, we included several objective diagnostic measurements alongside the self-report measurements, but the statistical power of our design was actually too small to make any solid conclusions based on the data. Lack of power is a problem that is difficult to overcome when performing pragmatic effectiveness trials. To perform additional analyses, a huge amount of participants is necessary to reach statistical power, which makes recruitment hazardous. Furthermore, although several studies have shown that a diagnosis can be obtained by phone (Donker et al., 2010; Evans et al., 2004; Rohde et al., 1997), there is always a risk of missing vital information that might have been detected in a face-to-face interview. A formal diagnostic classification applied in face-to-face screening would provide a more stringent and reliable measure. A third limitation in our measurements is the absence of competence measures. Competence measurements are required to assure that 
the researchers are following protocol, which, in turn, confirms and guarantees the quality of the treatment.

Lastly, MBIs - and specifically ACT - have been called transdiagnostic (Hayes et al., 2012; Newby et al., 2015), as they are effective for a broad range of disorders or symptoms and decrease psychological inflexibility as a risk factor for psychopathology (Chawla and Ostafin, 2007; Hayes et al., 2006; Hayes et al., 2004; Kashdan and Rottenberg, 2010; Roemer et al., 2005). Our trials and interventions were specifically focused on people with mild to moderate depressive symptoms. However, the concept of unified or transdiagnostic interventions has some advantages over treatments for specific disorders. One advantage is that recruitment is easier, and a larger study population helps to increase the study's statistical power. Transdiagnostic psychological interventions have been developed as a way of cutting across diagnostic categories, focusing on the commonalities across disorders instead of the differences (e.g., Barlow et al., 2004; Newby et al., 2015; Newby et al., 2016). The potential benefits for patients and clinicians are reduced waiting lists and management of comorbidity. Recent meta-analyses are encouraging and indicate that transdiagnostic interventions may result in similar outcomes when compared to disorder-specific treatments (McEvoy et al., 2009; Newby et al., 2016; Newby et al., 2015).

To summarize, future research might specifically focus on:

- Testing whether the observed beneficial effects of (online) MBIs are maintained over time.

- Using more sophisticated mediation designs to address temporal precedence of process variables, such as cross-lagged panel designs.

- Identifying specific moderators and predictors of the effects of (online) MBIs.

- Transcending the traditional RCT and including other types of trials, such as single case experimental design studies and separate analyses on adherers and nonadherers.

- Developing randomised controlled trials on transdiagnostic public mental health interventions.

\section{Conclusion}

To conclude, public mental health interventions have the potential to effectively promote mental health in an accessible way for people with mild to moderate depressive symptoms. This thesis describes six studies on the effectiveness of MBIs as public mental health interventions, and qualifies the use of (online) MBIs. Furthermore, this thesis also shows that web-based ACT interventions seem to be the treatment 
of choice as they are superior in effectiveness in the short-term, as well as acceptable and applicable. The theoretical framework of ACT has been confirmed, showing that increases in mindfulness and psychological flexibility mediate the effect on depressive symptoms. In addition, there seems to be no restrictions to the allocation of web-based $\mathrm{ACT}$, at least for the population of the study, making it easy to implement in regular care. While no true answer to what works best for whom exists, the best choice, based on the current knowledge, seems to be making available effective public mental health interventions and evolving them to best meet the needs of different users. As to whether human guidance is necessary, that remains a question for further exploration. While not all persons needing treatment for depression should necessarily be directed to an (online) MBI, clinicians can make solid decisions, weighing the advantages of online MBIs to the needs of their clients. 


\section{References}

A-Tjak, J. G. L., Davis, M. L., Morina, N., Powers, M. B., Smits, J. A. J. \& Emmelkamp, P. M. G. (2015). A meta-analysis of the efficacy of acceptance and commitment therapy for clinically relevant mental and physical health problems. Psychotherapy and Psychosomatics 84, 30-36.

Aardoom, J. J., Dingemans, A. E., Spinhoven, P., van Ginkel, J. R., de Rooij, M. \& van Furth, E. F. (2016). Web-Based Fully Automated Self-Help With Different Levels of Therapist Support for Individuals With Eating Disorder Symptoms: A Randomized Controlled Trial. Journal of Medical Internet Research 18, e159.

Abbott, R. A., Whear, R., Rodgers, L. R., Bethel, A., Thompson Coon, J., Kuyken, W., ... Dickens, C. (2014). Effectiveness of mindfulness-based stress reduction and mindfulness based cognitive therapy in vascular disease: A systematic review and meta-analysis of randomised controlled trials. Journal of Psychosomatic Research 76, 341-351.

Aldao, A. \& Nolen-Hoeksema, S. (2010). Specificity of cognitive emotion regulation strategies: A transdiagnostic examination. Behaviour Research and Therapy 48, 974-983.

American Mindfulness Research Association (2016). Mindfulness journal publications by year, 1980-2015.

Andersson, G. \& Cuijpers, P. (2009). Internet-based and other computerized psychological treatments for adult depression: A meta-analysis. Cognitive Behaviour Therapy 38, 196-205.

Andersson, G., Paxling, B., Wiwe, M., Vernmark, K., Felix, C. B., Lundborg, L., . . Carlbring, P. (2012). Therapeutic alliance in guided internet-delivered cognitive behavioural treatment of depression, generalized anxiety disorder and social anxiety disorder. Behaviour Research and Therapy 50, 544-50.

Andersson, G. \& Titov, N. (2014). Advantages and limitations of Internet-based interventions for common mental disorders. World Psychiatry 13, 4-11.

Andrews, G., Cuijpers, P., Craske, M. G., McEvoy, P. \& Titov, N. (2010). Computer therapy for the anxiety and depressive disorders is effective, acceptable and practical health care: A meta-analysis. PLOS ONE 5, el3196.

Andrews, G., Issakidis, C., Sanderson, K., Corry, J. \& Lapsley, H. (2004). Utilising survey data to inform public policy: Comparison of the cost-effectiveness of treatment of ten mental disorders. British Journal of Psychiatry 184, 526-533.

Arnberg, F. K., Linton, S. J., Hultcrantz, M., Heintz, E. \& Jonsson, U. (2014). Internet-delivered psychological treatments for mood and anxiety disorders: A systematic review of their efficacy, safety, and cost-effectiveness. PLoS ONE 9, eg8118.

Barlow, D. H., Allen, L. B. \& Choate, M. L. (2004). Toward a unified treatment for emotional disorders. Behavior Therapy 35, 205-230.

Baumeister, H., Reichler, L., Munzinger, M. \& Lin, J. (2014). The impact of guidance on Internet-based mental health interventions - A systematic review. Internet Interventions 1, 205-215.

Berger, T., Caspar, F., Richardson, R., Kneubühler, B., Sutter, D. \& Andersson, G. (2011). Internetbased treatment of social phobia: A randomized controlled trial comparing unguided with two types of guided self-help. Behaviour Research and Therapy 49, 158-169.

Bohlmeijer, E. T., Fledderus, M., Rokx, T. A. J. J. \& Pieterse, M. E. (2011). Efficacy of an early intervention based on acceptance and commitment therapy for adults with depressive symptomatology: Evaluation in a randomized controlled trial. Behaviour Research and Therapy 49, 62-67.

Bolier, L., Haverman, M., Kramer, J., Westerhof, G. J., Riper, H., Walburg, J. A., ... Bohlmeijer, E. (2013a). An internet-Based intervention to promote mental fitness for mildly depressed adults: Randomized controlled trial. Journal of Medical Internet Research 15.

Bolier, L., Haverman, M., Westerhof, G. J., Riper, H., Smit, F. \& Bohlmeijer, E. (2013b). Positive psychology interventions: A meta-analysis of randomized controlled studies. BMC Public Health 13, 119.

Bower, P. \& Gilbody, S. (2005). Stepped care in psychological therapies: Access, effectiveness and efficiency. Narrative literature review. British Journal of Psychiatry 186, 11-17. 
Carlson, L. E., Doll, R., Stephen, J., Faris, P., Tamagawa, R., Drysdale, E. \& Speca, M. (2013). Randomized Controlled Trial of Mindfulness-Based Cancer Recovery Versus Supportive Expressive Group Therapy for Distressed Survivors of Breast Cancer (MINDSET). Journal of Clinical Oncology 31, 3119-3126.

Carmody, J. \& Baer, R. A. (2008). Relationships between mindfulness practice and levels of mindfulness, medical and psychological symptoms and well-being in a mindfulness-based stress reduction program. Journal of Behavioral Medicine 31, 23-33.

Carmody, J. \& Baer, R. A. (2009). How long does a mindfulness-based stress reduction program need to be? A review of class contact hours and effect sizes for psychological distress. Journal of Clinical Psychology 65, 627-638.

Cavanagh, K., Strauss, C., Forder, L. \& Jones, F. (2014). Can mindfulness and acceptance be learnt by self-help?: A systematic review and meta-analysis of mindfulness and acceptance-based self-help interventions. Clinical Psychology Review 34, 118-129.

Chawla, N. \& Ostafin, B. (2007). Experiential avoidance as a functional dimensional approach to psychopathology: An empirical review. Journal of Clinical Psychology 63, 871-890.

Chiesa, A., Anselmi, R. \& Serretti, A. (2014). Psychological mechanisms of mindfulness-based interventions: What do we know? Holistic Nursing Practice 28, 124-148.

Chiesa, A., Serretti, A. \& Jakobsen, J. C. (2013). Mindfulness: Top-down or bottom-up emotion regulation strategy? Clinical Psychology Review 33, 82-96.

Christensen, H., Griffiths, K. M. \& Farrer, L. (2009). Adherence in internet interventions for anxiety and depression. Journal of Medical Internet Research 11, e13.

Coronado-Montoya, S., Levis, A. W., Kwakkenbos, L., Steele, R. J., Turner, E. H. \& Thombs, B. D. (2016). Reporting of Positive Results in Randomized Controlled Trials of Mindfulness-Based Mental Health Interventions. PLOS ONE 11, e0153220.

Cuijpers, P. \& Smit, F. (2004). Subthreshold depression as a risk indicator for major depressive disorder: A systematic review of prospective studies. Acta Psychiatrica Scandinavica 109, 325-331.

Cuijpers, P., van Straten, A., Andersson, G. \& van Oppen, P. (2008a). Psychotherapy for Depression in Adults: A Meta-Analysis of Comparative Outcome Studies. Journal of Consulting and Clinical Psychology 76, 909-922.

Cuijpers, P., Van Straten, A., Warmerdam, L. \& Smits, N. (2008b). Characteristics of effective psychological treatments of depression: A metaregression analysis. Psychotherapy Research 18, 225-236.

Cuijpers, P., Van Straten, A., Warmerdam, L. \& Van Rooy, M. J. (2010). Recruiting participants for interventions to prevent the onset of depressive disorders: Possibile ways to increase participation rates. BMC Health Services Research 10, 181.

De Graaf, L. E., Gerhards, S. A. H., Arntz, A., Riper, H., Metsemakers, J. F. M., Evers, S. M. A. A., . Huibers, M. J. H. (2009). Clinical effectiveness of online computerised cognitive-behavioural therapy without support for depression in primary care: Randomised trial. British Journal of Psychiatry 195, 73-80.

De Graaf, L. E., Gerhards, S. A. H., Arntz, A., Riper, H., Metsemakers, J. F. M., Evers, S. M. A. A., . . . Huibers, M. J. H. (2011). One-year follow-up results of unsupported online computerized cognitive behavioural therapy for depression in primary care: A randomized trial. Journal of Behavior Therapy and Experimental Psychiatry 42, 89-95.

Donker, T., van Straten, A., Marks, I. \& Cuijpers, P. (2010). Brief self-rated screening for depression on the Internet. Journal of Affective Disorders 122, 253-259.

Donkin, L., Christensen, H., Naismith, S. L., Neal, B., Hickie, I. B. \& Glozier, N. (2011). A Systematic Review of the Impact of Adherence on the Effectiveness of e-Therapies. Journal of Medical Internet Research 13, e52.

Driessen, E., Hollon, S. D., Bockting, C. L. H., Cuijpers, P. \& Turner, E. H. (2015). Does Publication Bias Inflate the Apparent Efficacy of Psychological Treatment for Major Depressive Disorder? A Systematic Review and Meta-Analysis of US National Institutes of Health-Funded Trials. PLoS ONE 10, eo137864. 
Evans, M., Kessler, D., Lewis, G., Peters, T. J. \& Sharp, D. (2004). Assessing mental health in primary care research using standardized scales: Can it be carried out over the telephone? Psychological Medicine 34, 157-162.

Eysenbach, G. (2005). The law of attrition. Journal of Medical Internet Research 7, e11.

Farb, N. A. S., Anderson, A. K. \& Segal, Z. V. (2012). The mindful brain and emotion regulation in mood disorders. Canadian Journal of Psychiatry 57, 70-77.

Fledderus, M., Bohlmeijer, E. T., Fox, J. P., Schreurs, K. M. G. \& Spinhoven, P. (2013). The role of psychological flexibility in a self-help acceptance and commitment therapy intervention for psychological distress in a randomized controlled trial. Behaviour Research and Therapy 51, 142-151.

Fledderus, M., Bohlmeijer, E. T., Pieterse, M. E. \& Schreurs, K. M. G. (2012). Acceptance and commitment therapy as guided self-help for psychological distress and positive mental health: A randomized controlled trial. Psychological Medicine 42, 485-495.

Fogg, B. J. (2003). Persuasive technology: using computers to change what we think and do. Morgan Kaufmann Publishers: Boston.

Forman, E. M., Chapman, J. E., Herbert, J. D., Goetter, E. M., Yuen, E. K. \& Moitra, E. (2012). Using Session-by-Session Measurement to Compare Mechanisms of Action for Acceptance and Commitment Therapy and Cognitive Therapy. Behavior Therapy 43, 341-354.

Forman, E. M., Herbert, J. D., Moitra, E., Yeomans, P. D. \& Geller, P. A. (2007). A randomized controlled effectiveness trial of acceptance and commitment therapy and cognitive therapy for anxiety and depression. Behavior Modification 31, 772-799.

Fry, J. P. \& Neff, R. A. (2009). Periodic prompts and reminders in health promotion and health behavior interventions: Systematic review. Journal of Medical Internet Research 11, e16.

Geschwind, N., Peeters, F., Huibers, M., Van Os, J. \& Wichers, M. (2012). Efficacy of mindfulnessbased cognitive therapy in relation to prior history of depression: Randomised controlled trial. British Journal of Psychiatry 201, 320-325.

Gilbody, S., Bower, P., Fletcher, J., Richards, D. \& Sutton, A. J. (2006). Collaborative care for depression: A cumulative meta-analysis and review of longer-term outcomes. Archives of Internal Medicine 166, 2314-2321.

Gould, J. D. \& Lewis, C. (1985). Designing for usability: Key principles and what designers think. Communications of the ACM 28, 300-311.

Grist, R. \& Cavanagh, K. (2013). Computerised cognitive behavioural therapy for common mental health disorders, what works, for whom under what circumstances? A systematic review and meta-analysis. Journal of Contemporary Psychotherapy 43, 243-251.

Gu, J., Strauss, C., Bond, R. \& Cavanagh, K. (2015). How do mindfulness-based cognitive therapy and mindfulness-based stress reduction improve mental health and wellbeing? A systematic review and meta-analysis of mediation studies. Clinical Psychology Review 37, 1-12.

Hacker, T., Stone, P. \& Macbeth, A. (2016). Acceptance and commitment therapy - Do we know enough? Cumulative and sequential meta-analyses of randomized controlled trials. Journal of Affective Disorders 190, 551-565.

Hadjistavropoulos, H. D., Pugh, N. E., Hesser, H. \& Andersson, G. (2016). Therapeutic Alliance in Internet-Delivered Cognitive Behaviour Therapy for Depression or Generalized Anxiety. Cllinical Psychology and Psychotherapy.

Hayes, S. C., Luoma, J. B., Bond, F. W., Masuda, A. \& Lillis, J. (2006). Acceptance and Commitment Therapy: Model, processes and outcomes. Behaviour Research and Therapy 44, 1-25.

Hayes, S. C., Strosahl, K., Wilson, K. G., Bissett, R. T., Pistorello, J., Toarmino, D., ... McCurry, S. M. (2004). Measuring experiential avoidance: A preliminary test of a working model. Psychological Record 54, 553-578.

Hayes, S. C., Strosahl, K. D. \& Wilson, K. G. (2012). Acceptance and Commitment Therapy: the Process and Practice of Mindful Change. Guilford Press: New York.

Hofmann, S. G., Sawyer, A. T., Fang, A. \& Asnaani, A. (2012). Emotion dysregulation model of mood and anxiety disorders. Depression and Anxiety 29, 409-416. 
Hofmann, S. G., Sawyer, A. T., Witt, A. A. \& Oh, D. (2010). The Effect of Mindfulness-Based Therapy on Anxiety and Depression: A Meta-Analytic Review. Journal of Consulting and Clinical Psychology 78, 169-183.

Hoge, E. A., Bui, E., Marques, L., Metcalf, C. A., Morris, L. K., Robinaugh, D. J., ... Simon, N. M. (2013). Randomized Controlled Trial of Mindfulness Meditation for Generalized Anxiety Disorder: Effects on Anxiety and Stress Reactivity. Journal of Clinical Psychiatry 74, 786-792.

Hölzel, B. K., Lazar, S. W., Gard, T., Schuman-Olivier, Z., Vago, D. R. \& Ott, U. (2011). How does mindfulness meditation work? Proposing mechanisms of action from a conceptual and neural perspective. Perspectives on Psychological Science 6, 537-559.

Hurling, R., Fairley, B. W. \& Dias, M. B. (2006). Internet-based exercise intervention systems: Are more interactive designs better? Psychology and Health 21, 757-772.

Jané-Llopis, E., Barry, M., Hosman, C. \& Patel, V. (2005). Mental health promotion works: a review. Promotion \& education Suppl 2, 9-25, 61, 67.

Kashdan, T. B. \& Ciarrochi, J. (2013). Mindfulness, acceptance, and positive psychology: The seven foundations for well-being. Context Press: Oakland, CA.

Kashdan, T. B. \& Rottenberg, J. (2010). Psychological flexibility as a fundamental aspect of health. Clinical Psychology Review 30, 865-878.

Kelders, S. M. (2012). Understanding adherence to web-based interventions. p. 250 . University of Twente: Enschede.

Kelders, S. M., Bohlmeijer, E. T., Pots, W. T. M. \& van Gemert-Pijnen, J. E. W. C. (2015). Comparing human and automated support for depression: Fractional factorial randomized controlled trial. Behaviour Research and Therapy 72, 72-80.

Kelders, S. M., Kok, R. N., Ossebaard, H. C. \& Van Gemert-Pijnen, J. E. W. C. (2012). Persuasive system design does matter: A systematic review of adherence to web-based interventions. Journal of Medical Internet Research 14, e152.

Kelders, S. M., T. Bohlmeijer, E. \& Van Gemert-Pijnen, J. E. W. C. (2013). Participants, usage, and use patterns of a web-based intervention for the prevention of depression within a randomized controlled trial. Journal of Medical Internet Research 15, e172.

Kemmeren, L. L., van Schaik, D. J., Riper, H., Kleiboer, A. M., Bosmans, J. E. \& Smit, J. H. (2016). Effectiveness of blended depression treatment for adults in specialised mental healthcare: study protocol for a randomised controlled trial. BMC Psychiatry 16, 113.

Keyes, C. L. M., Dhingra, S. S. \& Simoes, E. J. (2010). Change in level of positive mental health as a predictor of future risk of mental Illness. American Journal of Public Health 100, 2366-2371.

Khoury, B., Lecomte, T., Fortin, G., Masse, M., Therien, P., Bouchard, V., ... Hofmann, S. G. (2013). Mindfulness-based therapy: A comprehensive meta-analysis. Clinical Psychology Review 33, 763-771.

Khoury, B., Sharma, M., Rush, S. E. \& Fournier, C. (2015). Mindfulness-based stress reduction for healthy individuals: A meta-analysis. Journal of Psychosomatic Research 78, 519-528.

Kooistra, L. C., Wiersma, J. E., Ruwaard, J., van Oppen, P., Smit, F., Lokkerbol, J., . . Riper, H. (2014). Blended vs. face-to-face cognitive behavioural treatment for major depression in specialized mental health care: study protocol of a randomized controlled cost-effectiveness trial. BMC Psychiatry 14, 290.

Kujala, S. (2003). User involvement: A review of the benefits and challenges. Behaviour and Information Technology 22, 1-16.

Kuyken, W., Hayes, R., Barrett, B., Byng, R., Dalgleish, T., Kessler, D., ... Byford, S. (2015). Effectiveness and cost-effectiveness of mindfulness-based cognitive therapy compared with maintenance antidepressant treatment in the prevention of depressive relapse or recurrence (PREVENT): a randomised controlled trial. The Lancet 386, 63-73.

Kuyken, W., Warren, F. C., Taylor, R. S., Whalley, B., Crane, C., Bondolfi, G., ... Dalgleish, T. (2016). Efficacy of Mindfulness-Based Cognitive Therapy in Prevention of Depressive Relapse: An Individual Patient Data Meta-analysis From Randomized Trials. JAMA Psychiatry 73, 565-74. 
Kuyken, W., Watkins, E., Holden, E., White, K., Taylor, R. S., Byford, S., ... Dalgleish, T. (2010). How does mindfulness-based cognitive therapy work? Behaviour Research and Therapy 48, 1105-1112.

Lamers, S. M. A., Westerhof, G. J., Glas, C. A. W. \& Bohlmeijer, E. T. (2015). The bidirectional relation between positive mental health and psychopathology in a longitudinal representative panel study. Journal of Positive Psychology 10, 553-560.

Lappalainen, R., Lehtonen, T., Skarp, E., Taubert, E., Ojanen, M. \& Hayes, S. C. (2007). The impact of $\mathrm{CBT}$ and $\mathrm{ACT}$ models using psychology trainee therapists: a preliminary controlled effectiveness trial. Behavior Modification 31, 488-511.

Ma, S. H. \& Teasdale, J. D. (2004). Mindfulness-Based Cognitive Therapy for Depression: Replication and Exploration of Differential Relapse Prevention Effects. Journal of Consulting and Clinical Psychology 72, 31-40.

McEvoy, P. M., Nathan, P. \& Norton, P. J. (2009). Efficacy of Transdiagnostic Treatments: A Review of Published Outcome Studies and Future Research Directions. Journal of Cognitive Psychotherapy 23, 20-33.

Meulenbeek, P., Willemse, G., Smit, F., Van Balkom, A., Spinhoven, P. \& Cuijpers, P. (2010). Early intervention in panic: Pragmatic randomised controlled trial. British Journal of Psychiatry 196, 326-331.

Mohr, D. C., Cuijpers, P. \& Lehman, K. (2011). Supportive Accountability: A Model for Providing Human Support to Enhance Adherence to eHealth Interventions. Journal of Medical Internet Research 13, eзo.

Mohr, D. C., Duffecy, J., Ho, J., Kwasny, M., Cai, X., Burns, M. N. \& Begale, M. (2013). A Randomized Controlled Trial Evaluating a Manualized TeleCoaching Protocol for Improving Adherence to a Web-Based Intervention for the Treatment of Depression. PLOS ONE 8, e70086.

Mohr, D. C., Spring, B., Freedland, K. E., Beckner, V., Arean, P., Hollon, S. D., ... Kaplan, R. (2009). The selection and design of control conditions for randomized controlled trials of psychological interventions. Psychotherapy and Psychosomatics 78, 275-284.

Muñoz, R. F., Cuijpers, P., Smit, F., Barrera, A. Z. \& Leykin, Y. (2010). Prevention of major depression. Annual Review of Clinical Psychology 6, 181-212.

Newby, J. M., McKinnon, A., Kuyken, W., Gilbody, S. \& Dalgleish, T. (2015). Systematic review and meta-analysis of transdiagnostic psychological treatments for anxiety and depressive disorders in adulthood. Clinical Psychology Review 40, 91-110.

Newby, J. M., Twomey, C., Yuan Li, S. S. \& Andrews, G. (2016). Transdiagnostic computerised cognitive behavioural therapy for depression and anxiety: A systematic review and meta-analysis. Journal of Affective Disorders 199, 30-41.

Niles, A. N., Burklund, L. J., Arch, J. J., Lieberman, M. D., Saxbe, D. \& Craske, M. G. (2014). Cognitive mediators of treatment for social anxiety disorder: Comparing acceptance and commitment therapy and cognitive-behavioral therapy. Behavior Therapy 45, 664-677.

Noar, S. M., Benac, C. N. \& Harris, M. S. (2007). Does Tailoring Matter? Meta-Analytic Review of Tailored Print Health Behavior Change Interventions. Psychological Bulletin 133, 673-693.

Nolen-Hoeksema, S. (2000). The role of rumination in depressive disorders and mixed anxiety/depressive symptoms. Journal of Abnormal Psychology 109, 504-511.

Norton, P. J. \& Paulus, D. J. (2015). Toward a Unified Treatment for Emotional Disorders: Update on the Science and Practice. Behavior Therapy, in press.

Oinas-Kukkonen, H. (2010). Behavior change support systems: A research model and agenda. In Lecture Notes in Computer Science (including subseries Lecture Notes in Artificial Intelligence and Lecture Notes in Bioinformatics), pp. 4-14.

Oinas-Kukkonen, H. \& Harjumaa, M. (2009). Persuasive systems design: Key issues, process model, and system features. Communications of the Association for Information Systems 24, 485-500.

Olatunji, B. O., Naragon-Gainey, K. \& Wolitzky-Taylor, K. B. (2013). Specificity of Rumination in Anxiety and Depression: A Multimodal Meta-Analysis. Clinical Psychology: Science and Practice 20, 225-257. 
Öst, L. G. (2014). The efficacy of Acceptance and Commitment Therapy: An updated systematic review and meta-analysis. Behaviour Research and Therapy 61, 105-121.

Pagliari, C. (2007). Design and evaluation in ehealth: Challenges and implications for an interdisciplinary field. Journal of Medical Internet Research 9, e15.

Piet, J. \& Hougaard, E. (2011). The effect of mindfulness-based cognitive therapy for prevention of relapse in recurrent major depressive disorder: A systematic review and meta-analysis. Clinical Psychology Review 31, 1032-1040.

Postel, M. G., Witting, M. \& Van Gemert-Pijnen, J. E. (2015). Blended care in mental health care [Blended behandeling in de geestelijke gezondheidszorg]. Directieve Therapie 33, 210-221.

Pots, W. T. M., Fledderus, M., Meulenbeek, P. A. M., Klooster ten, P. M., Schreurs, K. M. \& Bohlmeijer, E. T. (2015). Acceptance and commitment therapy as a web-based intervention for depressive symptoms: randomised controlled trial. In The British Journal of Psychiatry.

Preschl, B., Maercker, A. \& Wagner, B. (2011). The working alliance in a randomized controlled trial comparing online with face-to-face cognitive-behavioral therapy for depression. BMC Psychiatry $11,189$.

Rashid, T. (2009). Positive interventions in clinical practice. Journal of Clinical Psychology 65, 461-466.

Rheker, J., Andersson, G. \& Weise, C. (2015). The role of "on demand" therapist guidance vs. no support in the treatment of tinnitus via the internet: A randomized controlled trial. Internet Interventions 2, 189-199.

Richards, D. \& Richardson, T. (2012). Computer-based psychological treatments for depression: A systematic review and meta-analysis. Clinical Psychology Review 32, 329-342.

Riper, H., van Ballegooijen, W., Kooistra, L., de Wit, J. \& Donker, T. (2013). Preventie \& eMental-health. Onderzoek dat leidt, technologie die verleidt, preventie die bereikt en beklijft. Vrije Universiteit Amsterdam: Amsterdam.

Ritterband, L. M., Cox, D. J., Gordon, T. L., Borowitz, S. M., Kovatchev, B. P., Walker, L. S. \& Sutphen, J. L. (2006). Examining the added value of audio, graphics, and interactivity in an internet intervention for pediatric encopresis. Children's Health Care 35, 47-59.

Roemer, L., Salters, K., Raffa, S. D. \& Orsillo, S. M. (2005). Fear and avoidance of internal experiences in GAD: Preliminary tests of a conceptual model. Cognitive Therapy and Research 29, 71-88.

Rohde, P., Lewinsohn, P. M. \& Seeley, J. R. (1997). Comparability of telephone and face-to-face interviews in assessing axis I and II disorders. American Journal of Psychiatry 154, 1593-1598.

Ruiz, F. J. (2010). A review of acceptance and commitment therapy (ACT) empirical evidence: Correlational, experimental psychopathology, component and outcome studies. International Journal of Psychology and Psychological Therapy 10, 125-162.

Ruwaard, J. \& Kok, R. N. (2015). Wild West eHealth: Time to hold our horses? The European Health Psychologist 17, 45-49.

Ryff, C. D. (2014). Psychological well-being revisited: advances in the science and practice of eudaimonia. Psychotherapy and Psychosomatics 83, 10-28.

Segal, Z. V., Williams, J. M. G. \& Teasdale, J. D. (2002). Mindfulness-based Cognitive Therapy for Depression: A New Approach to Preventing Relapse. The Guilford Press: New York.

Shafran, R., Clark, D. M., Fairburn, C. G., Arntz, A., Barlow, D. H., Ehlers, A., ... Wilson, G. T. (2009). Mind the gap: Improving the dissemination of CBT. Behaviour Research and Therapy 47, 902-909.

Spinhoven, P., Drost, J., de Rooij, M., van Hemert, A. M. \& Penninx, B. W. J. H. (2016). Is Experiential Avoidance a Mediating, Moderating, Independent, Overlapping, or Proxy Risk Factor in the Onset, Relapse and Maintenance of Depressive Disorders? Cognitive Therapy and Research 40, 150-163.

Strauss, C., Cavanagh, K., Oliver, A. \& Pettman, D. (2014). Mindfulness-based interventions for people diagnosed with a current episode of an anxiety or depressive disorder: A meta-analysis of randomised controlled trials. PLOS ONE 9, eg6no.

Strecher, V. J., McClure, J. B., Alexander, G. L., Chakraborty, B., Nair, V. N., Konkel, J. M., ... Pomerleau, O. F. (2008). Web-Based Smoking-Cessation Programs. Results of a Randomized Trial. American Journal of Preventive Medicine 34, 373-381. 
Tate, D. F., Jackvony, E. H. \& Wing, R. R. (2006). A randomized trial comparing human e-mail counseling, computer-automated tailored counseling, and no counseling in an internet weight loss program. Archives of Internal Medicine 166, 1620-1625.

Teasdale, J. D., Moore, R. G., Hayhurst, H., Pope, M., Williams, S. \& Segal, Z. V. (2002). Metacognitive awareness and prevention of relapse in depression: Empirical evidence. Journal of Consulting and Clinical Psychology 70, 275-287.

Teasdale, J. D., Segal, Z. \& Williams, J. M. G. (1995). How does cognitive therapy prevent depressive relapse and why should attentional control (mindfulness) training help? Behaviour Research and Therapy 33, 25-39.

Teasdale, J. D., Segal, Z. V., Williams, J. M. G., Ridgewaya, V. A., Soulsby, J. M. \& Lau, M. A. (200o). Prevention of relapse/recurrence in major depression by mindfulness-based cognitive therapy. Journal of Consulting and Clinical Psychology 68, 615-623.

Trompetter, H. R., Bohlmeijer, E. T., Veehof, M. M. \& Schreurs, K. M. G. (2014). Internet-based guided self-help intervention for chronic pain based on Acceptance and Commitment Therapy: A randomized controlled trial. Journal of Behavioral Medicine $38,66-80$.

Turner, E. H., Matthews, A. M., Linardatos, E., Tell, R. A. \& Rosenthal , R. (2008). Selective Publication of Antidepressant Trials and Its Influence on Apparent Efficacy. New England Journal of Medicine 358, 252-260.

Van Den Broeck, K., Remmen, R., Vanmeerbeek, M., Destoop, M. \& Dom, G. (2016). Collaborative care regarding major depressed patients: A review of guidelines and current practices. Journal of Affective Disorders 200, 189-203.

van der Vaart, R., Witting, M., Riper, H., Kooistra, L., Bohlmeijer, E. T. \& van Gemert-Pijnen, L. J. (2014). Blending online therapy into regular face-to-face therapy for depression: content, ratio and preconditions according to patients and therapists using a Delphi study. BMC Psychiatry 14,355 .

Van Der Zanden, R., Kramer, J., Gerrits, R. \& Cuijpers, P. (2012). Effectiveness of an online group course for depression in adolescents and young adults: A randomized trial. Journal of Medical Internet Research 14, e86.

van Gemert-Pijnen, J. E., Nijland, N., van Limburg, M., Ossebaard, H. C., Kelders, S. M., Eysenbach, G. \& Seydel, E. R. (2011). A holistic framework to improve the uptake and impact of eHealth technologies. Journal of Medical Internet Research 13, en1.

Vøllestad, J., Nielsen, M. B. \& Nielsen, G. H. (2012). Mindfulness- and acceptance-based interventions for anxiety disorders: A systematic review and meta-analysis. British Journal of Clinical Psychology 51, 239-260.

Webb, T. L., Joseph, J., Yardley, L. \& Michie, S. (2010). Using the internet to promote health behavior change: a systematic review and meta-analysis of the impact of theoretical basis, use of behavior change techniques, and mode of delivery on efficacy. Journal of Medical Internet Research 12, e4.

Wentzel, J., van der Vaart, R., Bohlmeijer, E. T. \& van Gemert-Pijnen, J. E. (2016). Mixing Online and Face-to-Face Therapy: How to Benefit From Blended Care in Mental Health Care. JMIR Ment Health 3 , eg.

Wicksell, R. K., Olsson, G. L. \& Hayes, S. C. (2011). Mediators of change in Acceptance and Commitment Therapy for pediatric chronic pain. Pain 152, 2792-2801.

Wildeboer, G., Kelders, S. M. \& van Gemert-Pijnen, J. E. W. C. (2016). The relationship between persuasive technology principles, adherence and effect of web-Based interventions for mental health: A meta-analysis. International Journal of Medical Informatics, in press.

Wood, A. M. \& Joseph, S. (2010). The absence of positive psychological (eudemonic) well-being as a risk factor for depression: A ten year cohort study. Journal of Affective Disorders 122, 213-217.

Zainal, N. Z., Booth, S. \& Huppert, F. A. (2013). The efficacy of mindfulness-based stress reduction on mental health of breast cancer patients: A meta-analysis. Psycho-Oncology 22, 1457-1465.

Zimmerman, M., McGlinchey, J. B., Posternak, M. A., Friedman, M., Attiullah, N. \& Boerescu, D. (2006). How Should Remission From Depression Be Defined? The Depressed Patient's Perspective. American Journal of Psychiatry 163, 148-150. 
CHAPTER 8 

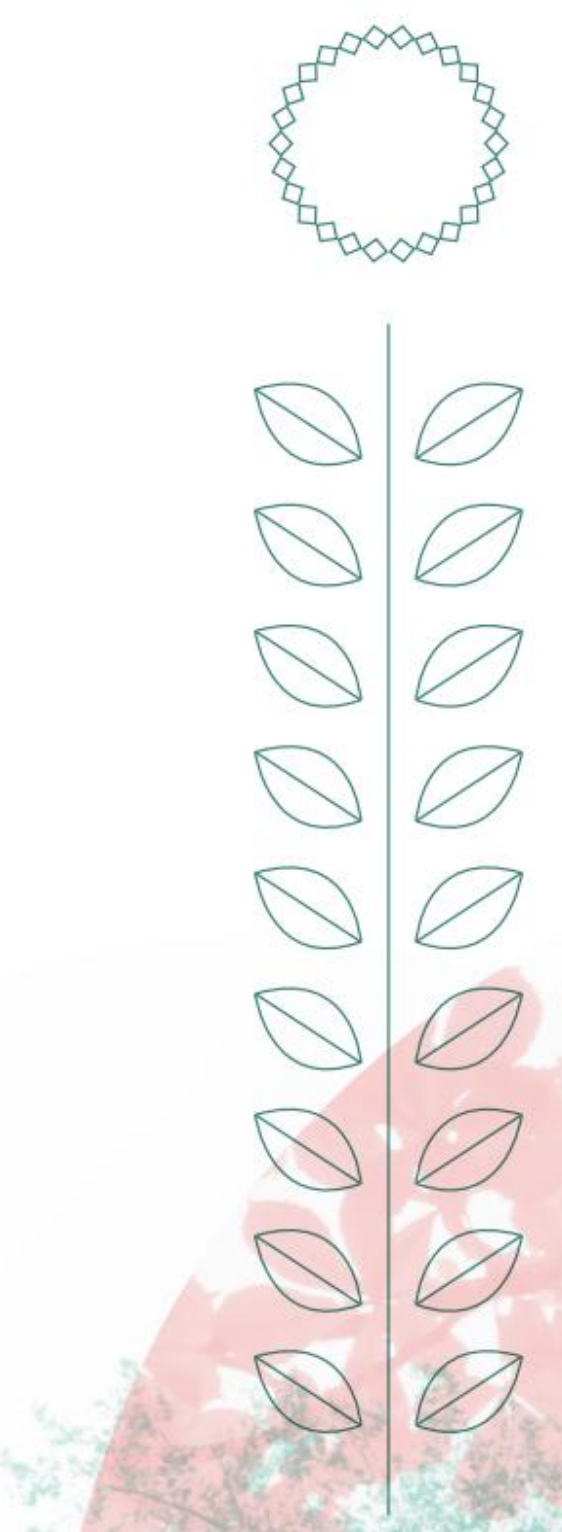

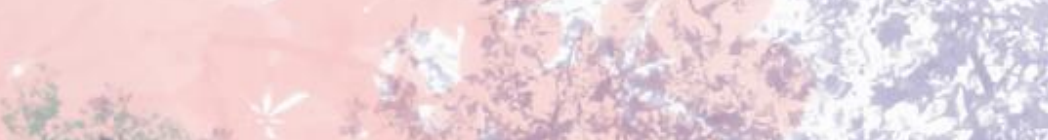
is $3 x^{2}$

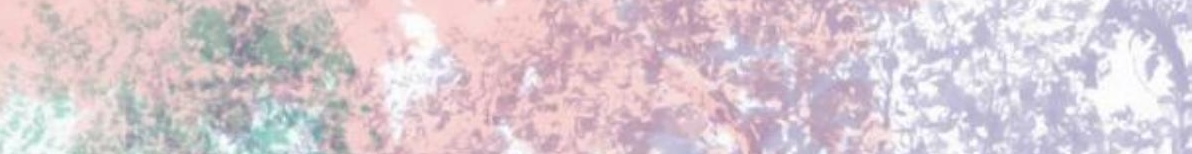

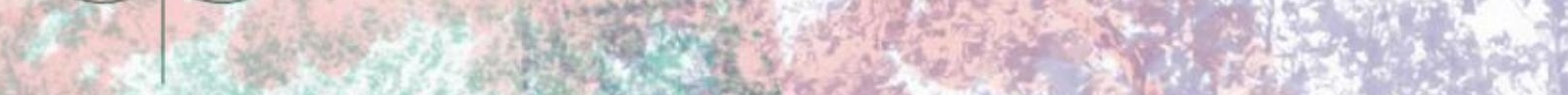
(5)

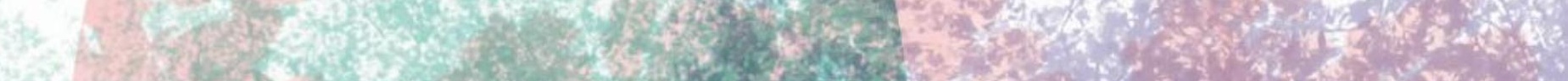

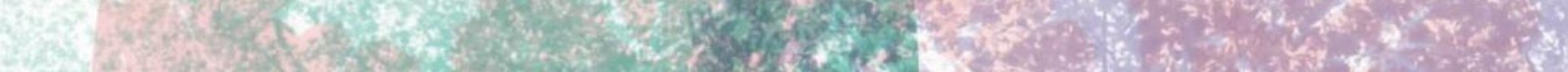

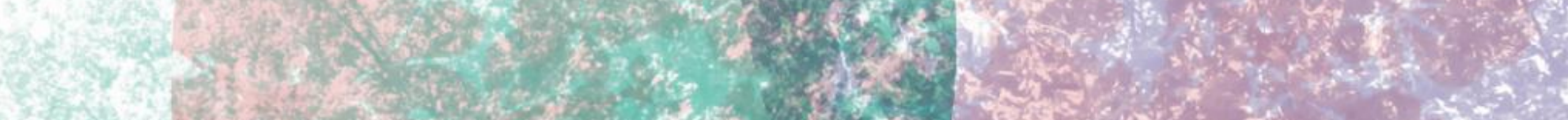

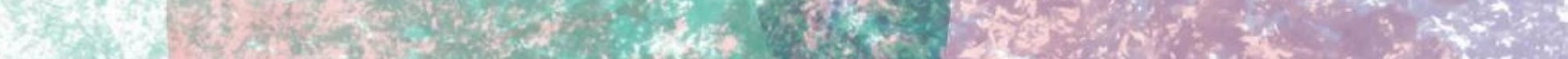

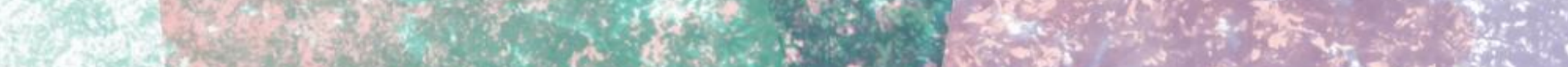

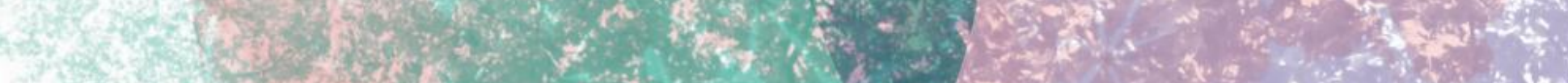

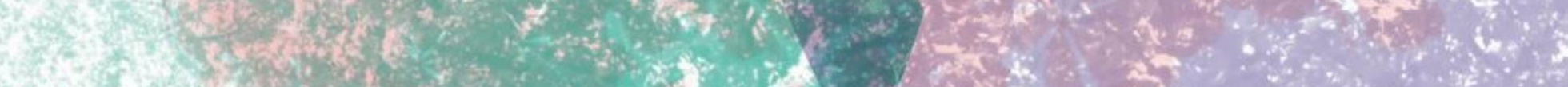

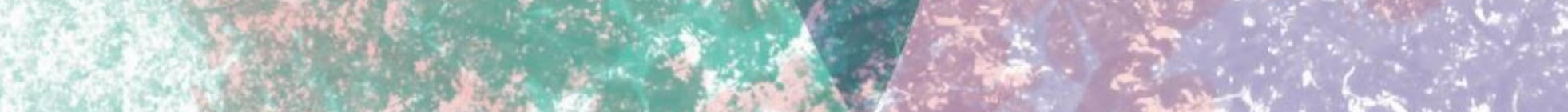
3050 (3)

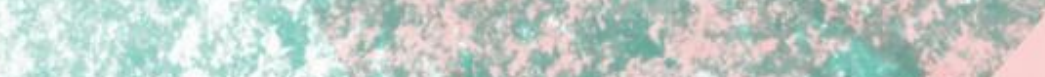

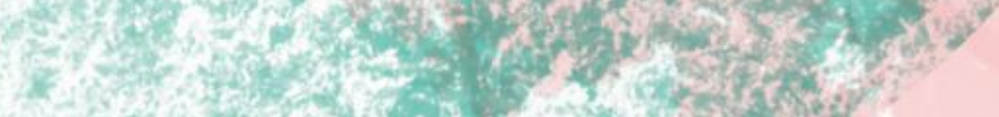
i

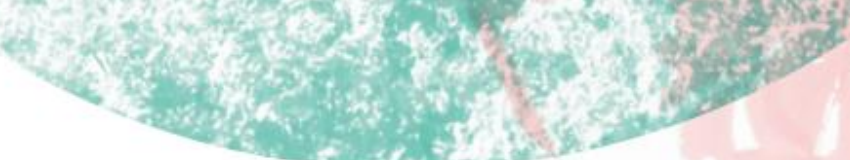




\section{Samenvatting}

Depressie behoort tot de top vijf van de ziekten met de hoogste ziektelast in Nederland en heeft een grote negatieve invloed op de kwaliteit van leven van de mensen die eraan lijden. Daarnaast gaat depressie gepaard met hoge kosten, zowel door zorgkosten als door productieverlies. Uit onderzoek blijkt dat één van de belangrijkste risicofactoren voor het krijgen van een depressie op latere leeftijd de aanwezigheid is van depressieve klachten. Het is dus van belang om deze mensen met beginnende klachten op tijd een behandeling aan te bieden, zodat het ontwikkelen of het verergeren van een depressie kan worden voorkomen. Alhoewel er effectieve preventieve interventies in omloop zijn, is de impact van deze preventieve interventies op de incidentie van depressie laag. Uit onderzoek blijkt dat stigmatisatie, hoge drempels om in zorg te komen en een slecht bereik van effectieve behandelingen bepalende factoren zijn.

In Hoofdstuk 1, de inleiding van dit proefschrift, werden de doelen van dit proefschrift en de context waarin de studies zijn uitgevoerd beschreven. Dit proefschrift richt zich op de ontwikkeling en effectiviteit van preventieve behandelingen die toegankelijk en aantrekkelijk zijn, zoals web-based interventies of online behandelingen. Met een online behandeling kunnen mensen snel en effectief aan hun depressieve klachten werken, zonder dat ze de drempel van een geestelijke gezondheidsinstelling hoeven te passeren. Daarnaast blijkt het uit onderzoek van belang dat behandelingen naast het verminderen van klachten zich ook richten op het versterken van welbevinden. Voor dit doel richtte dit proefschrift zich op preventieve interventies gebaseerd op mindfulness en Acceptance and Commitment Therapie (ACT). Mindfulness is het vermogen om zonder oordeel je gedachten, gevoelens of lichamelijke sensaties van moment tot moment op te merken. Een specifieke vorm van behandeling gebaseerd op mindfulness is Mindfulness-based Cognitive Therapy (MBCT). MBCT is een therapie waarbij mindfulness oefeningen geïntegreerd zijn met Cognitieve Gedrags Therapie (CGT). Het doel van MBCT is niet de inhoud van gedachten te veranderen, maar de verhouding ten opzichte van de gedachten. ACT is een relatief nieuwe vorm van cognitieve gedragstherapie die acceptatie en mindfulness strategieën combineert met commitment en gedragsveranderingsstrategieën. Het doel van ACT is het vergroten van psychologische flexibiliteit, de bereidheid om in contact te komen met innerlijke ervaringen, belangrijke waarden in je leven te herkennen en je te committeren aan gedrag dat passend is bij die waarden. Ondanks dat interventies gebaseerd op mindfulness en ACT potentie hebben als preventieve interventie voor depressie, is er weinig wetenschappelijk onderzoek gedaan naar de effectiviteit van deze preventieve interventies. Het aanbieden van (online) interventies gebaseerd op mindfulness zou het bereik van preventieve interventies voor depressie kunnen 
vergroten. Met dit proefschrift werden de volgende drie doelen beoogd: 1) onderzoek naar de effectiviteit van MBCT als preventieve interventie voor mensen met milde tot matige depressieve klachten; 2) onderzoek naar het potentieel van online interventies gebaseerd op mindfulness (MBIs) en de effectiviteit op depressie, angst, stress en welbevinden; 3) onderzoek naar ontwikkeling en (kosten)effectiviteit van een online interventie gebaseerd op ACT en mindfulness genaamd "Voluit Leven".

Het eerste doel, onderzoek naar de effectiviteit van Mindfulness-Based Cognitive Therapy (MBCT) als preventieve interventie voor mensen met depressieve klachten, werd beschreven in Hoofdstuk 2. Dit hoofdstuk beschreef de resultaten van een pragmatische gerandomiseerde gecontroleerde trial waarbij de effectiviteit van MBCT als preventieve interventie voor mensen met subklinische of milde tot matige depressieve klachten werd getoetst. MBCT is oorspronkelijk ontwikkeld voor preventie van terugval van mensen met een recidiverende depressieve stoornis in remissie als een 8-weekse groepscursus, die mindfulness oefeningen met cognitieve technieken combineert. Mindfulness heeft de afgelopen jaren veel belangstelling gekregen, zodanig dat er ook veel MBCT trainingen werden aangeboden binnen de eerstelijnszorg en de basis GGZ voor mensen met subklinische depressieve klachten. Alhoewel er veel wetenschappelijk onderzoek was gedaan naar de effectiviteit van MBCT voor mensen met een recidiverende stoornis in remissie, was er nauwelijks onderzoek naar de effectiviteit van MBCT bij de doelgroep mensen met milde tot matige depressieve klachten. Veel mensen met deze subklinische klachten zijn beperkt in hun tijd vanwege verplichtingen op het werk of sociaal, wat een drempel zou kunnen zijn qua tijdsinvestering of bereidheid deel te nemen. Om de MBCT training passend te maken voor deze doelgroep, mensen met milde tot matige depressieve klachten, werd de training aangepast in intensiteit. De originele mindfulness oefeningen van 45 minuten werden gereduceerd tot 15 minuten, en de sessies werden bekort tot 1,5 uur in plaats van de originele 2,5 uur. De inhoud van de training werd identiek gehouden door deze uit te spreiden over 1 in plaats van 8 bijeenkomsten. Vergeleken met een wachtlijst groep $(n=75)$ was deze preventieve MBCT interventie $(n=76)$ effectief in het verminderen van depressieve klachten, angstklachten en experientiële vermijding. Dit effect bleef behouden na een follow-up van drie maanden. De kans op een klinisch significante verandering op depressieve klachten was bovendien significant hoger in de MBCT groep. Naast effectiviteit is het van belang te kijken wat de werkzame processen van een interventie zijn. Uit de mediatie analyses bleek dat zowel het vergroten van psychologische flexibiliteit als mindfulness de effecten van MBCT op depressieve klachten medieerde. Dit betekent dat het bevorderen van een open attitude tegenover gedachten, gevoelens en lichamelijke sensaties en het vergroten van psychologische flexibiliteit belangrijke werkingsmechanismen zijn in het effect 
van MBCT op depressieve klachten. De resultaten van deze studie suggereren dat het aanbieden van een preventieve MBCT interventie met een lage intensiteit aan mindfulness oefeningen effectief kan zijn voor mensen met milde tot matige depressieve klachten. Dit is van belang aangezien de beschikbaarheid van een effectieve interventie, opgezet vanuit de positieve psychologie, het bereik van preventieve interventies kan vergoten. Daarnaast zou met het verminderen van depressieve klachten en het vergroten van de positieve geestelijke gezondheid het risico op een full-blown depressie verkleind kunnen worden.

Het tweede doel van dit proefschrift was onderzoeken of online interventies gebaseerd op mindfulness (MBIs) effectief kunnen zijn in het verminderen van psychische en stress klachten. Hoofdstuk 3 beschreef een meta-analyse naar studies die de effectiviteit van MBIs onderzochten. Er werden met behulp van een systematische literatuurstudie 15 gerandomiseerde studies geselecteerd met een totaal van 2360 deelnemers. Van iedere studie werd een gestandaardiseerde pre-post effectmaat berekend. Deze effecten zijn gepoold tot een totaal effect van depressie, angst, stress, subjectief welbevinden, en mindfulness. Op alle maten werd een significant effect gevonden (0.22-0.51), met het grootste effect op stress (0.51). Voor depressieve klachten werd een kleine effect size gevonden (0.29). Voor stress en mindfulness gold dat de effect sizes significant hoger werden wanneer er alleen werd gekeken naar begeleide online interventies in vergelijking met onbegeleide online interventies. Daarnaast leek het aantal sessies het effect op stress te voorspellen: hoe meer sessies, hoe hoger het effect. De kwaliteit van de studies bleek niet significant gerelateerd aan het effect. De resultaten lieten zien dat online MBIs potentie hebben om psychische en stress klachten te verbeteren. Dit is een belangrijke bevinding, aangezien online interventies makkelijk toegankelijk zijn, goed te implementeren zijn in de zorg, en minder stigmatiserend zijn. MBIs zouden dus een goed alternatief kunnen zijn voor traditionele interventies, die voornamelijk aansluiten op het ziektemodel. Gezien de gelimiteerde studies die geïncludeerd konden worden en het risico op positieve publicatiebias, wordt aanbevolen dat verder onderzoek nodig is om de bewijskracht van online MBIs te vergroten.

Het derde doel van dit proefschrift was onderzoek naar ontwikkeling en (kosten) effectiviteit van een online preventieve interventie gebaseerd op ACT en mindfulness, genaamd "Voluit Leven", voor volwassenen met milde tot matige depressieve klachten. Dit onderzoek werd opgesplitst in vier onderzoeksvragen, die afzonderlijk werden behandeld in de hoofdstukken 4, 5, 6 en 7. In Hoofdstuk 4 werd een onderzoek beschreven naar de ontwikkeling van "Voluit Leven" als preventieve online interventie voor de preventie van depressie. Online interventies worden vaak ad hoc ontwikkeld, 
waarbij er weinig aandacht wordt besteed aan de technologie. Uit onderzoek blijkt dat dit één van de redenen is dat online interventies een hoge uitval hebben. Het doel van dit onderzoek was een gebruiksvriendelijke interventie te ontwikkelen, die voldeed aan de waarden van de gebruikers en andere stakeholders door hun feedback mee te nemen in het evalueren van het proces van ontwikkeling. De ontwikkeling werd beschreven aan de hand van de Center for eHealth Research and Disease Management (CeHRes) Roadmap, waarbij verschillende fasen werden doorlopen, geëvalueerd en bijgesteld. Methodieken in de fasen waren onder andere een literatuur scan, interviews, rapid prototyping, usability inspectie en evaluatie, en afstemming van de behoeften op het ontwerp en de uitvoering. Door samen met de gebruikers en andere stakeholders de technologie te ontwikkelen, konden we het systeem, de inhoud en de service van de online interventie aanpassen aan de wensen en behoeften van alle stakeholders. Door het proces van de ontwikkeling te evalueren werd het belang van het betrekken van gebruikers, experts, onderzoekers, designers en technische programmeurs goed zichtbaar, en werd betrokkenheid gecreëerd.

In Hoofdstuk 5 werden de resultaten van het onderzoek naar de effectiviteit van de "Voluit Leven" als online interventie beschreven. Er werd een gerandomiseerde gecontroleerde studie gedaan met drie controlegroepen: "Voluit Leven" ( $n=82$ ) werd vergeleken met zowel een actieve controlegroep (Expressief Schrijven als online interventie; $n=67)$, als een wachtlijst controlegroep $(n=87)$. De resultaten van deze klinische trial toonden aan dat mensen die "Voluit Leven" hadden gevolgd een significante vooruitgang lieten zien over een periode van zes maanden wat betreft depressieve klachten, angstklachten, positieve geestelijke gezondheid, psychologische flexibiliteit en mindfulness, vergeleken met beide controlegroepen. Extra analyses lieten zien dat het grootste effect te vinden was op 3 maanden na baseline (0.56-0.36), maar dat op de lange termijn (follow-up 6 en 12 maanden) de effecten van de condities gelijk waren. Zowel Expressief Schrijven als de wachtlijstcontrolegroep liet een spontane verbetering van klachten zien na 6 maanden, een gegeven dat bekend is in de literatuur (de meeste depressies verdwijnen spontaan na 6 maanden). Daarnaast liet Expressief Schrijven ook een significante verbetering zien op psychologische flexibiliteit en mindfulness vergeleken met de wachtlijst controlegroep, wat zou kunnen impliceren dat Expressief Schrijven wellicht een potentieel effectieve interventie is in plaats van alleen een aandachtscontrole. Echter, het feit dat "Voluit Leven" effectiever is op de korte termijn is van belang, aangezien we uit onderzoek weten dat de aanwezigheid van depressieve klachten een risicofactor is voor het ontwikkelen van een depressie en gepaard gaat met hoge economische kosten. Vroeg ingrijpen is dus relevant en noodzakelijk. De adherentie (therapietrouw) van "Voluit Leven" was hoog: $84 \%$ van de mensen hadden minimaal zes sessies gevolgd, en $73 \%$ had alle negen lessen afgerond. Klinisch relevante verandering op depressieve klachten was bovendien na 
drie maanden significant hoger in "Voluit Leven" (54\%), in vergelijking met Expressief Schrijven ( $31 \%$ ) en de wachtlijst controlegroep (26\%).

Naast het uitzoeken óf interventies effect hebben is het ook belangrijk te kijken naar hóe ze effect hebben, en voor wíe. In Hoofdstuk 6 werd het onderzoek beschreven naar mediatoren, de werkingsmechanismen van verandering, gedurende de behandeling. Daarnaast onderzochten we of "Voluit Leven" als online interventie effectief was voor alle deelnemers, door moderatoren en predictoren van verandering gedurende de RCT te toetsen. De uitkomsten lieten allereerst zien dat, wanneer "Voluit Leven" vergeleken werd met de wachtlijstcontrole groep, psychologische flexibiliteit een centraal mechanisme van verandering was, conform het model van ACT. Daarnaast bleek mindfulness eveneens het effect van "Voluit Leven" op depressie te medieren. Wanneer gekeken werd naar moderatie en predictie, werden de effecten van "Voluit Leven" op depressieve klachten niet beïnvloed door demografische factoren, psychische klachten, diagnostische classificatie, of comorbiditeit. De enige moderator die gevonden werd was het hebben van een psychische stoornis op baseline als voorspeller voor een grotere verbetering op depressieve klachten na 12 maanden, voor "Voluit Leven" versus Expressief Schrijven. Daarnaast werd gevonden dat psychische klachten en positieve geestelijke gezondheid op baseline voorspellers waren voor verbetering op depressieve klachten, ongeacht de groep waar mensen zich in bevonden. Concluderend kan gesteld worden dat er vooralsnog geen reden is mensen te excluderen voor deelname aan "Voluit Leven" als online interventie, alhoewel wanneer de ernst van de depressieve klachten erger is wellicht "Voluit Leven" effectiever is op de lange termijn dan Expressief Schrijven. Gezien de exploratieve analyses moeten de resultaten echter met voorzichtigheid geïnterpreteerd worden, en wordt geadviseerd verder onderzoek te doen waarbij de mediatieen moderatieanalyses als hoofdanalyses worden meegenomen.

De laatste studie naar "Voluit Leven" als online interventie betreft een kosteneffectiviteitsstudie. In Hoofdstuk 7 werden de resultaten van een kosteneffectiviteits- en kostenutiliteitsanalyse op de data van "Voluit Leven" beschreven, om de verhouding tussen kosten en effectiviteit en tussen kosten en gezondheidswinst te onderzoeken. De resultaten lieten zien dat "Voluit Leven" dominant was ten opzichte van Expressief Schrijven op 6 maanden. Dit effect verdween op 12 maanden follow-up. In vergelijking met de wachtlijst controlegroep was de kans dat "Voluit Leven" superieur was $39 \%$ en $33 \%$, voor respectievelijk kostenefectiviteit en kostenutiliteit. Gevoeligheidsanalyses lieten zien dat met realistisch lagere interventiekosten "Voluit Leven" dominant was ten opzichte van beide controlegroepen.

Dit proefschrift is een van de eerste onderzoeken die de effectiviteit laat zien van preventieve (online) interventies gebaseerd op ACT en mindfulness voor mensen 
met milde tot matige depressieve klachten. In Hoofdstuk 8 werden de belangrijkste bevinden en implicaties van dit proefschrift beschreven en bediscussieerd. Concluderend kan gesteld worden dat (online) MBIs als preventieve interventies potentie hebben als aantrekkelijke en effectieve behandeling voor mensen met subklinische depressieve klachten. 

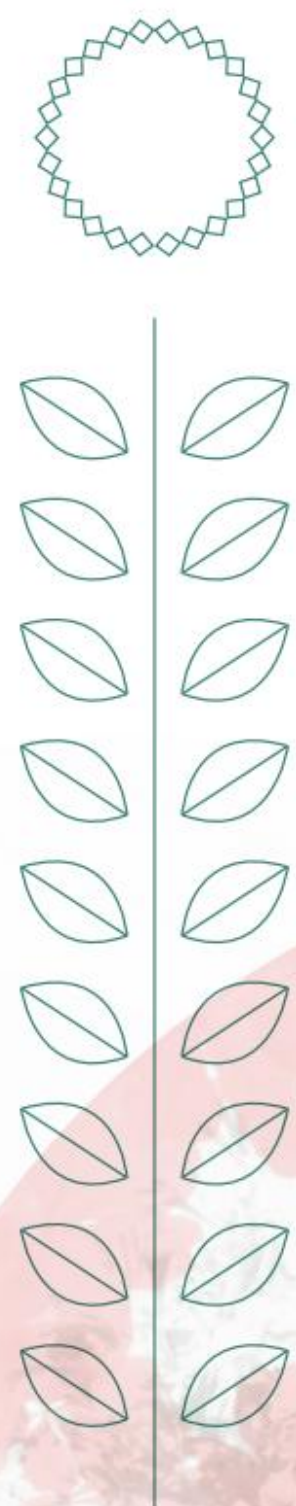

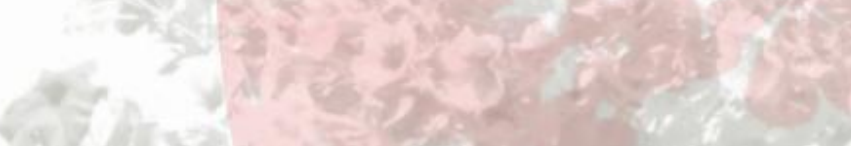

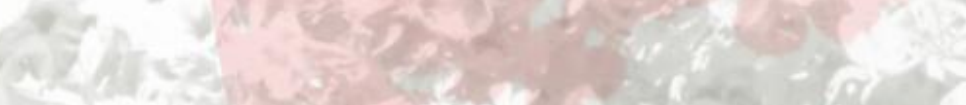

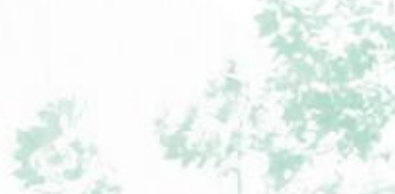

to.

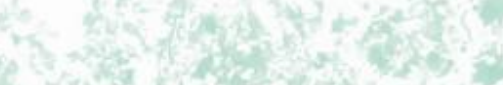

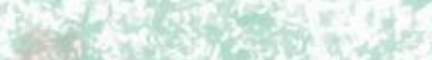

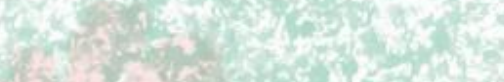

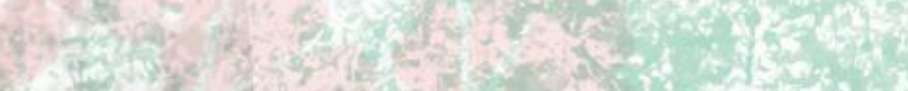

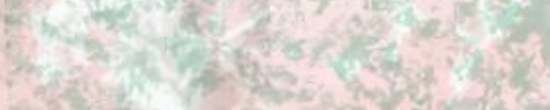

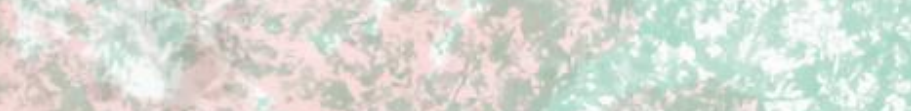

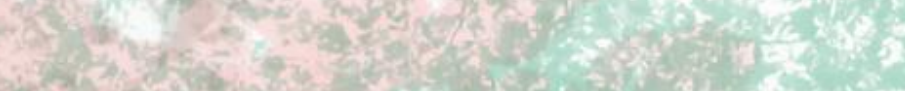

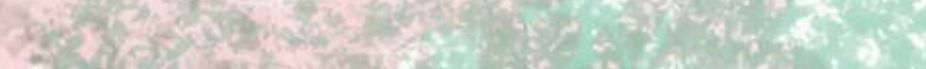

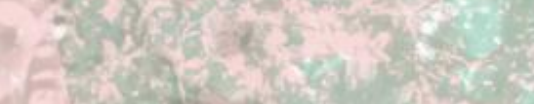
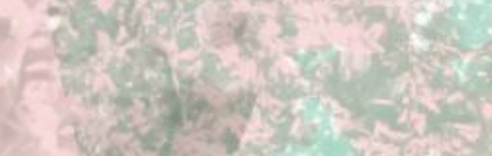

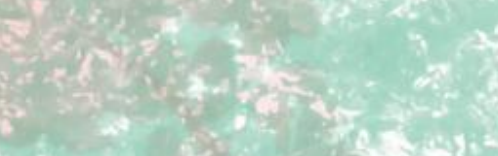

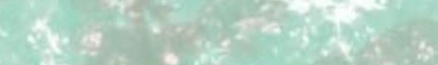

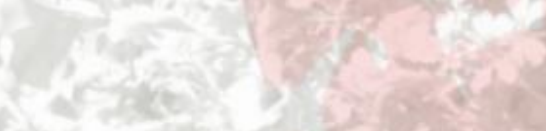
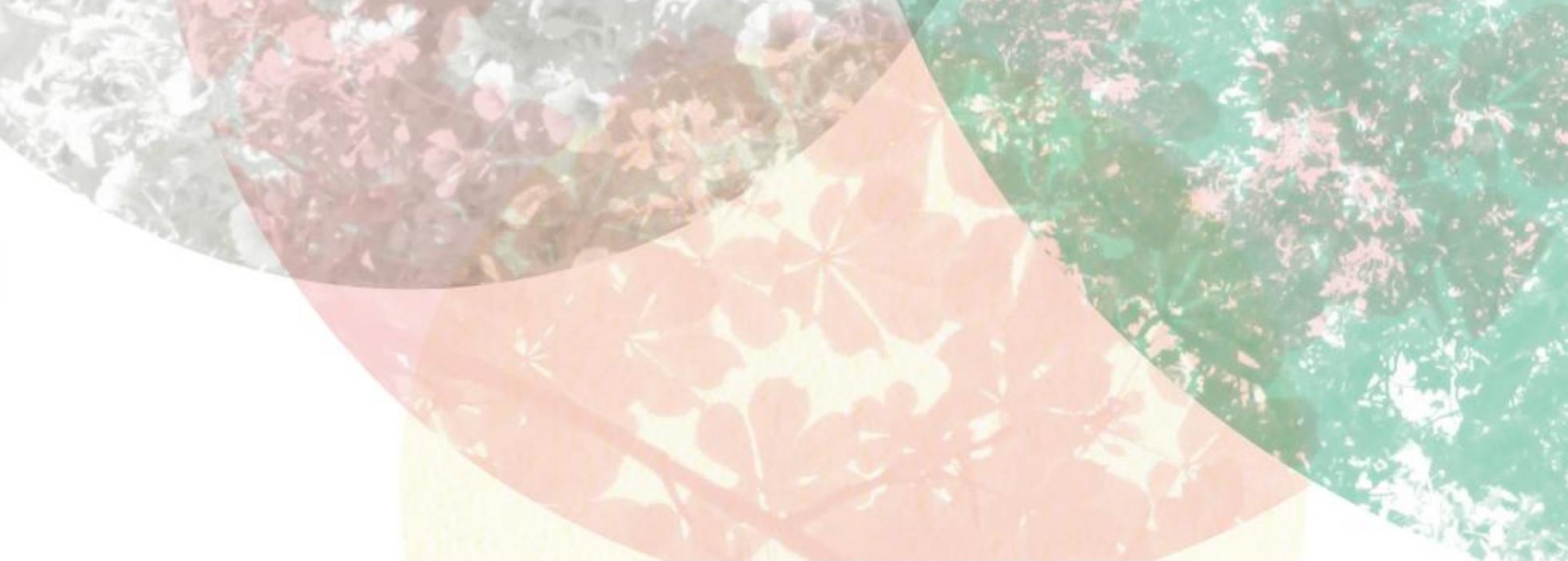


\section{Dankwoord}

Voluit leven in eenzaamheid is moeilijker dan met mensen om je heen. Zonder jullie allemaal was ik niet blijven staan.

Depressieve klachten is niemand vreemd, en zeker ook mij niet. Als ik zei dat ik me somber voelde, dan werd me gevraagd wat voor onderzoek ik ook alweer doe, "iets met mindfulness toch? Dan kan je dat toch gaan doen?”. Dat riep weerstand op, maar tegelijkertijd ook de bewustwording van het geluk dat ik heb om dit onderzoek te mogen doen. Onderzoek naar een thema waar ik al jaren interesse in heb, samen met mijn snode plan om de gezondheidszorg te transformeren. Het leven draait niet om geluk, en leed hoort bij het leven. Die balans was af en toe wat verstoord, maar het is tevens een enorme leerschool geweest aan emotieregulatie en planningsvaardigheden (naast al die wetenschappelijke kennis over goed onderzoek doen). Voor die leerschool ben ik zowel Dimence als Ernst Bohlmeijer zeer dankvoor.

Ernst, je bent bijzonder leermeester en mens. Je bent iemand met visie zonder controlerend te willen zijn, geeft anderen vertrouwen in hun kunnen en zet ze op het rechte pad. Je weet me altijd een niveau hoger te tillen en hebt voortdurend nieuwe kennis paraat, wat enorm inspirerend is. Je hebt me gezien en me de kans gegeven bezig te zijn met het onderwerp dat ik al jaren in mijn hart heb. Je geeft me de ruimte om eigenwijs en zelfs perfectionistisch te zijn, maar met een bescheiden lach begrens je dat ook. Dank je wel dat je mijn promotor wilde zijn, en ik verheug me op onze verdere samenwerking.

Karlein, je bent eigenlijk al langer mijn leermeester en hebt me zien groeien in mijn hele carrière zo ongeveer. Bij het Roessingh kon ik nog niet goed afscheid nemen van mijn neuropsycholoog-kant en koos ik voor de CVA-revalidatie i.p.v. de pijn-revalidatie waar jij werkte. Ik herinner me jou in de sollicitatie en vooral dat ik dacht dat het prettig en leerzaam zou zijn om met je samen te werken. Het samenwerken met jou heb ik later ingehaald door je te strikken mijn $\mathrm{N}=1$ supervisor te zijn. Dat we elkaar weer troffen bij de UT en dat je mijn tweede promotor werd, was een vooropgezet plan :). Je bent een van de weinige clinici die én een scherpe theoretische kijk heeft op psychologie, én een steengoede psycholoog is met hart voor haar cliënten. Wat heerlijk dat ik met je kan reflecteren op therapieën en theorieën, en ook nog gewoon mens mag zijn. Ik hoop dat ik je nog veel vaker kan strikken voor gezamenlijke klussen.

Peter, als co-promotor kon je me bijstaan in het hele traject. Dank voor je kritische en scherpe blik die mijn proefschrift heeft doen verbeteren en mij doen groeien. Het helpt om iemand naast je te hebben die wellicht net zo (of iets minder) perfectionistisch en gedetailleerd is als ik. 
Graag wil ik ook alle leden van de promotiecommissie bedanken: Prof. dr. Philip Spinhoven, Prof. dr. Jan Spijker, dr. Linda Bolier, Prof. dr. Lisette van Gemert-Pijnen en Prof. dr. Job van der Palen. Bedankt voor jullie bereidheid deel te nemen in de promotiecommissie.

Dit proefschrift is tot stand gekomen mede dankzij Dimence, een werkgever waar ik met plezier al jaren werk en die zeker ook medewerkers de gelegenheid geeft zich te ontwikkelen. Ik dank Dimence, en in het bijzonder Herma van der Wal, voor de mogelijkheid dat ik op de Universiteit Twente kon promoveren.

Binnen Dimence zijn mijn leidinggevenden en collega's altijd steunend geweest in dit proces, zowel in vrijheden verlenen als in een troostende en opbeurende woorden. Gelukkig kunnen hulpverleners dat goed!

Mijn collega's en ex-collega's van de vakgroep Psychologie, Gezondheid \& Technologie aan de UT, wat zijn jullie ook fijn! Wat heerlijk om altijd binnen te kunnen lopen om bobo-vragen te stellen, of te klagen over hoe zwaar onderzoek is, of om te kletsen. Saskia, Hester, Marijke Schotanus, Marion Spijkerman, Martine Fledderus en Peter ten Klooster, dank voor de fijne samenwerking en de feedback op de artikelen en mijn stress :). Dank Martine Veehof, die aan de start van mijn promotietraject me de fijne kneepjes van de kunst van wetenschapsonderzoek heeft geleerd.

Dank ook Prof. dr. Filip Smit voor de fijne samenwerking en positiviteit en enthousiasme in het schrijven van een kosteneffectiviteitsstudie.

Een aantal andere mensen in het bijzonder wil ik nog bedanken. Marre, wat heerlijk dat ik altijd bij je kon komen om te werken en mezelf te zijn. Ina, Lot, Farid en Lieke, dank jullie wel voor de feedback bij het lezen en het verdragen van mijn gesteun. Lot en Ilona, wat super dat jullie mijn paranimfen willen zijn! Het voelt zo vertrouwd jullie naast me te hebben. En al mijn lieve vrienden waarmee ik ga chillen in de sauna, ga feesten, lunchen, hangen op de bank of ga wandelen, dank jullie wel! Dat heeft me helpen gronden, lachen en genieten van het leven.

Pap en mam, wat fijn dat we meer tijd samen hebben sinds ik op de UT werk. Ik geniet van de donderdagen of vrijdagen dat ik binnen kan vallen en aanschuiven aan tafel, kletsen over alles wat ik meemaak in mijn leven en met mijn promotietraject. Dat we dat vooral maar nog veel dagen mogen doen.

Merijn, ik ben zo blij dat we elkaar hebben gevonden. Voluit leven is niet zo moeilijk met jou. Volop genieten van de kleine dingen met jou maakt dat ik me thuis voel in mezelf. Jij verwarmt mijn hart. 

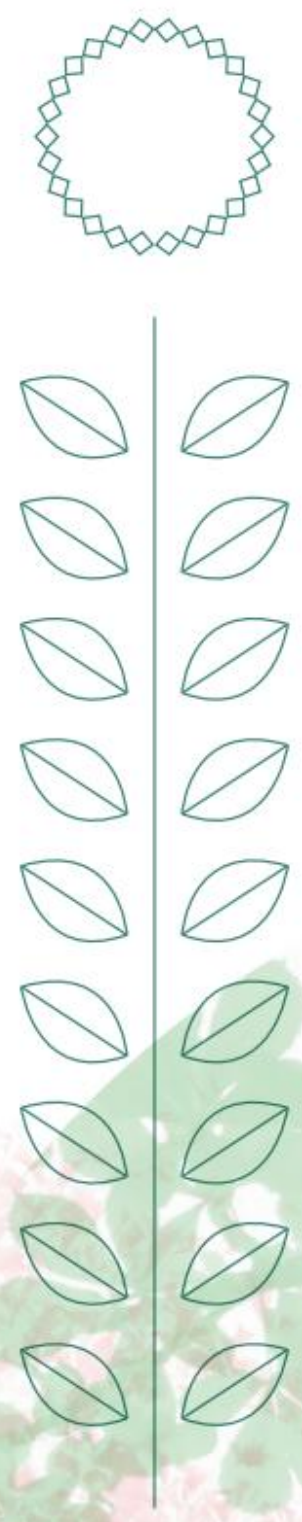


\section{Curriculum Vitae}




\section{Curriculum Vitae}

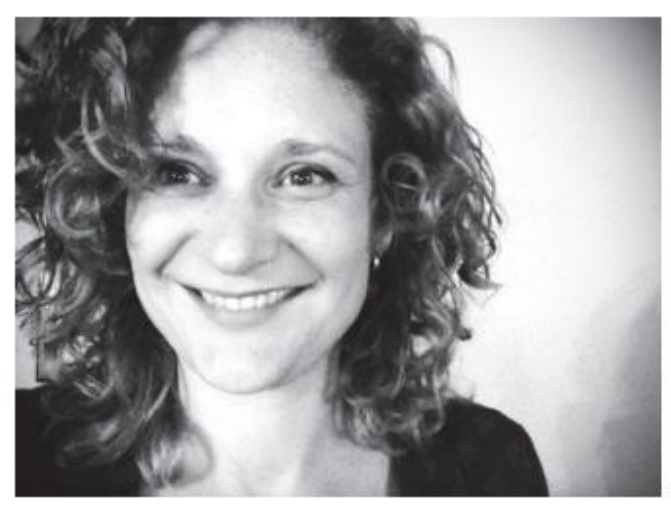

Wendy Pots is an experienced clinical psychologist with over 15 years of experience in the field of behavioural and cognitive therapy. She was born in Enschede in The Netherlands on the 4 th of June 1973. Wendy graduated from high school (Atheneum) at the Jacobus College in Enschede, and proceeded a study in Psychology at the University of Utrecht. She received her Bachelors degree in 1992, and followed a Master in neuropsychology and clinical psychology. She graduated in 1998 and found a job at Mediant, a mental health centre in Enschede, where she could work as a psychologist at a long-stay department. She was selected a year later at Mediant for a two-year training to get a licenced degree as psychologist (GZ-psycholoog). She has worked in different primary and secondary care settings, working with populations that face trauma, depression, anxiety and personality disorders. She worked as a cognitive behaviour therapist at Visie, a private practice in Deventer, and as a neuropsychologist at 't Roessingh, a centre for rehabilitation in Enschede. In 2008 she started working at Dimence as a cognitive behaviour therapist at the Depression \& Anxiety Department in Almelo. From May 2009 she combined her work as psychologist there with lecturing and research at the University of Twente. Her PhD project started in 2010. During her PhD project she also obtained a licensed Clinical Psychology degree (Klinisch Psycholoog) at Dimence. Her expertise is the treatment of complex trauma and cluster $C$ personality disorders. In her work with clients she uses key therapy techniques like Cognitive Behaviour Therapy (CBT), Acceptance and Commitment Therapy (ACT), Mindfulness-Based Cognitive Therapy (MBCT), Schema-Focused Cognitive Therapy and Eye Movement Desensitization and Reprocessing (EMDR). Currently, she is the Head of the Complex Trauma Department at Dimence in Almelo, and works as an Assistent Professsor at the department of Psychology, Health \& Technology at the University of Twente. 


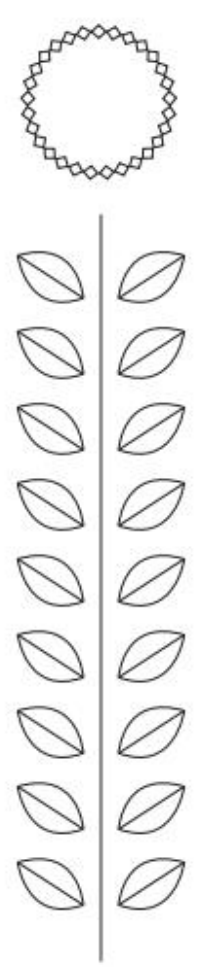






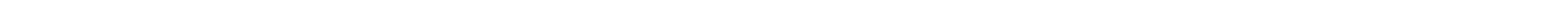

